Incorporation of $\mathscr{E P D M}$ into $\mathcal{N} R / \mathcal{B R}$ is a means to achieve non-staining ozone resistance for tire sidewall applications. Jowever, due to incompatibility of the elastomers and heterogeneous filler distribution in each of the rubber phases, the mechanical properties aeteriorate. The objective of the project covered in this thesis is

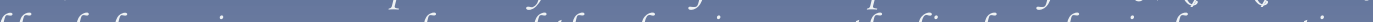
blends by various approaches and therefore improve the final mechanical properties of well to create a better understanding to this system.
EPDM-Rubber in Blends with NR/BR-Elastomers for Ozone-Resistant Tyre Sidewall Applications

New approaches for improved mechanical properties

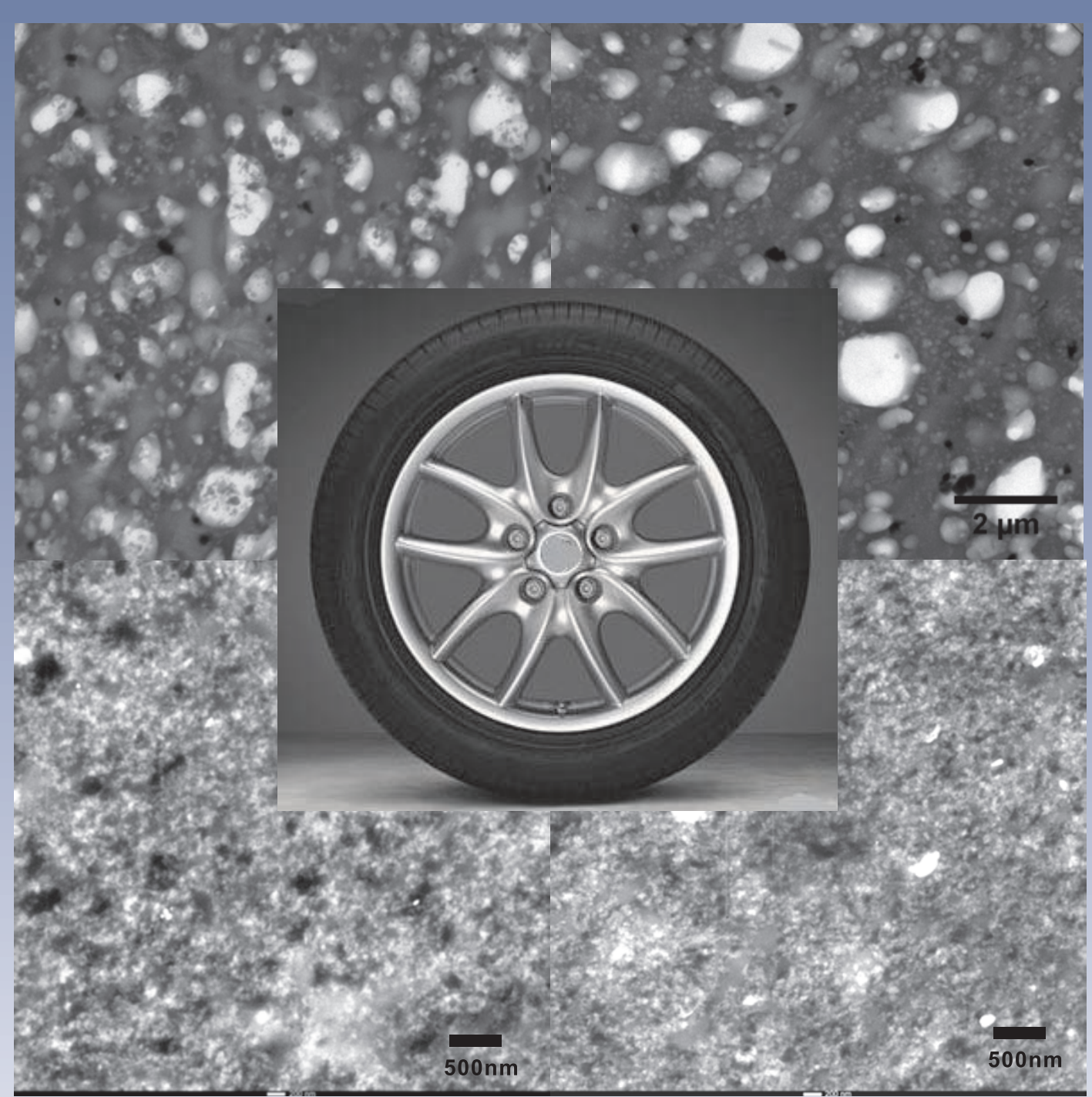

\section{Invitation}

You are cordially invited to the public defense of my PhD thesis entitled:

EPDM-Rubber in

Blends with NR/BR-

Elastomers for Ozone-

Resistant Tyre Sidewall Applications

On October 8th, 2009 at $13: 15 \mathrm{hrs}$

In Collegzaal 2, De Spiegel University of Twente, the Netherlands

At 13:00 hrs I will give a short introduction to the contents of my thesis.

A reception will follow after the ceremony.

Hongmei Zhang mayer028@hotmail.com

Paranimfen:

Jacob Lopulissa J.S.Lopulissa@ctw.utwente.n Kannika Sahakaro K.Sahakaro@ctw.utwente.n 
EPDM-Rubber in Blends with NR/BR-Elastomers for OzoneResistant Tyre Sidewall Applications

New Approaches for Improved Mechanical Properties 


\section{3\% DPI \\ DUTCH PQLYME ESTITHE}

The studies described in this thesis are part of the Research Programme of the Dutch Polymer Institute (DPI), P.O. Box 902, 5600 AX Eindhoven, The Netherlands, under project nr. \#356.

EPDM-Rubber in Blends with NR/BR-Elastomers for Ozone-Resistant Tyre Sidewall Applications: New Approaches for Improved Mechanical Properties

By Hongmei Zhang

Ph.D. thesis, University of Twente, Enschede, the Netherlands, 2009-8-5

With references - With summary in English and Dutch

Copyright (C) Hongmei Zhang, 2009-8-5

All rights reserved

Cover design by Hongmei Zhang

Printed by Print Partners Ipskamp, P.O. Box 333, 7500 AH, Enschede, the Netherlands

ISBN 978-90-365-2894-8 


\title{
EPDM-RUBBER IN BLENDS WITH NR/BR-ELASTOMERS FOR OZONE-RESISTANT TYRE SIDEWALL APPLICATIONS
}

NEW APPROACHES FOR IMPROVED MECHANICAL PROPERTIES

\section{DISSERTATION}

\author{
to obtain \\ the degree of doctor at the University of Twente, \\ on the authority of the rector magnificus, \\ prof. dr. H. Brinksma, \\ on account of the decision of the graduation committee, \\ to be publicly defended \\ on Thursday, 8 October at 13:15 hrs.
}

by

Hongmei Zhang

born on 28 January 1981

in Jiangsu, China 
This dissertation has been approved by:

Promotor : prof. dr. ir. J.W.M. Noordermeer Assistant promotor : dr. R.N. Datta 


\section{Table of Contents}

Chapter 1 General Introduction 1

Chapter 2 Mixing, Curing and Reinforcement of Dissimilar Rubbers for 7 Tyre Sidewall Applications: A Literature Review

Chapter 3 Studies on EPDM Modification by N-Chlorothio-N-Butyl- 35 Benzenesulphonamide

Chapter 4 Maleic-Anhydride Grafted EPM as Compatibilising Agent in 53 NR/BR/EPDM Blends: Part I. Preparation, Cure Characteristics and Mechanical Properties

Chapter 5 Maleic-Anhydride Grafted EPM as Compatibilising Agent in 75 NR/BR/EPDM Blends: Part II. Mechanistic Aspects

Chapter 6 Modification of Maleic Anhydride Grafted EPM with Sulphur Containing Chemicals Aiming at Improved Co-curing

Chapter 7 Studies on EPDM Modification by CBS, PNO and CLD

Chapter 8 Modification of EPDM with Alkylphenol Polysulphide for Use in Tyre Sidewalls: Part I. Mechanical Properties

Chapter 9 Modification of EPDM with Alkylphenol Polysulphide for Use in 133 Tyre Sidewalls: Part II. Mechanistic and Morphological Characterisations

Summary

Samenvatting

Symbols and Abbreviations

Curriculum Vitae 



\section{Chapter 1}

\section{General Introduction}

A historic overview and a general introduction into rubber blends are given in this chapter. The aim of this research and the structure of the thesis are stated. 


\subsection{Introduction}

Rubber is a fantastic material and is widely used in our normal lives due to its special characteristic: visco-elasticity. Use of a single rubber is rarely adequate for manufacturing of rubber products. Therefore, blends of rubbers are achieving more and more technological and commercial interest since they provide an acceptable technological process for accessing properties not available in a single elastomer. The potentially improved properties include chemical, physical and processing benefits. Changing intramolecular composition, such as making block copolymers, is a way to achieve tunable properties as well. However, this is limited by available synthesis processes. Intermolecular changes, such as adjusting composition or distribution of components in blends, are not limited by such synthetic limitations and are commercially preferred.

The start of elastomer blends can be traced back to $19^{\text {th }}$ century. Christopher Nickles patented the blending of gutta percha (trans-1,4-polyisoprene) into caoutchouc (cis-1,4-polyisoprene) for producing book bindings in 1845. In an 1846 patent, Charles Hancock made a much broader discussion of the gutta percha/caoutchouc blends for balancing composition and properties. From 1910 to 1918, Fritz Hofmann and his team developed the first synthetic elastomers and prepared blends of the new synthetic rubber poly(2,3-dimethyl butadiene) with natural rubber. Blend materials became commercial and were used by German rubber product manufactures during World War I.

From the 1920s onwards, more new synthetic rubbers were developed, such as polysulfide (Thiokol), polychloroprene (Duprene/Neoprene), polybutadiene (Buna), butadiene/acrylonitrile copolymer (Buna N/Perbunan), polyisobutylene (Oppanol) and butadiene/styrene copolymer (Buna S). Stocklin and Konrad patented their work on blending Buna $\mathrm{N}$ and polysulfide rubber in 1934. Nowak and Hofmeier described the performance of various synthetic rubber blends as, Buna S/Buna N, Buna S/Oppanol and Buna N/Oppanol in a paper in 1938. From that time on, more and more work has been carried out for rubber blends ${ }^{1}$.

Today the use of rubber blends is more widespreaded in applications, including belts, hoses, footwear and especially tyres and tyre related products. A tyre is an assembly of a series of parts, each of which has a specific function in the service and performance of the product. Table 1.1 lists the important components of tyres and the typical blends used for them². 
Table 1.1 Rubber Blends in Automotive Tyres.

\begin{tabular}{lll}
\hline \multicolumn{1}{c}{ Component } & \multicolumn{1}{c}{ Passenger tyres } & \multicolumn{1}{c}{ Truck tyres } \\
\hline Tread & SBR-BR & NR-BR or SBR-BR \\
Belt & NR & NR \\
Carcass & NR-SBR-BR & NR-BR \\
Black sidewall & NR-SBR or NR-BR & NR-BR \\
Inner liner & NR-SBR-IIR & NR-IIR \\
\hline
\end{tabular}

It is known that most elastomers are immiscible, because mixing is endothermic and the entropic contribution is small. Fortunately, miscibility is not a requirement for most applications. Homogeneity at a fairly fine level is sufficient for optimum properties and some degree of microheterogeneity can preserve the individual properties of each rubber component.

The most important aspects influencing the properties of rubber blends are cure compatibility and homogeneity of filler distribution. Covulcanisation of the components in the rubber blends is very important, because it contributes to a homogeneous crosslink distribution and adhesion between the different rubber phases. However, each elastomer has its own affinity for particular curing ingredients and has different reaction rates during the vulcanisation, which leads to cure incompatibility when the elastomer blends are vulcanised by the action of the same curing ingredients $s^{3-6}$. Due to the different levels of unsaturation and polarity for dissimilar rubbers, fillers also prefer to migrate into the higher polarity phases. All of these will finally result in over-curing and over-reinforcement of one phase but less curing and less reinforcement of the other, resulting in poor overall properties.

\subsection{Aim of this research}

Blending of saturated ethylene-propylene-diene rubber (EPDM) with highly unsaturated rubbers, such as natural rubber (NR) and (butadiene rubber) BR, is a rapidly developing area especially for tyre sidewall applications, where the saturated elastomer can be considered as a polymeric antioxidant for the diene rubbers ${ }^{7}$. However, vulcanisates of such elastomer blends are generally poor in mechanical properties. These undesirable phenomena are generally the result of the thermodynamic incompatibility of these two types of rubber, cure incompatibility and heterogeneous filler distribution in each of the rubber phases. However, despite these problems, blending of EPDM with highly unsaturated elastomers still attracts lots of interest as such blends may provide a range of applications, especially in tyre sidewalls. 
The objective of the project covered in this thesis is to overcome these incompatibility and reinforcement problems of the NR/BR/EPDM blends by various approaches and therefore improve the final mechanical properties of such blends. Besides the mechanical properties, the mechanisms involved are studied in the present work as well.

\subsection{Structure of this thesis}

A literature review on EPDM in blends with NR/BR for tyre sidewall applications is presented in Chapter 2. This chapter focuses on the various research approaches published in the field of blending, curing and reinforcement of dissimilar rubbers, as well as on the mechanism of ozone protection by incorporation of EPDM in the tyre sidewall application.

In Chapter 3, N-Chlorothio-N-Butyl-Benzenesulphonamide (CTBBS) is used as modification agent for EPDM in order to enhance the compatibility of EPDM with NR/BR. Reaction mechanisms are proposed based on the CTBBS modification on three different types of EPDM.

Chapter 4 describes the addition of Maleic-Anhydride modified EPM (MAHEPM) into NR/BR/EPDM blends as compatibilising agent. Straight EPM an N-phenylp-phenylenediamine (NPPDA) modified MAH-EPM (NPD-EPM) are also studied for comparison. The mechanical properties of the blends are described in this chapter. The mechanical properties are improved by the addition of MAH-EPM as compatibilising agent. Chapter $\mathbf{5}$ discusses the mechanistic aspects of those improvements.

In Chapter 6, based on the work described in Chapters 4 and 5, MAH-EPM is further modified by sulphur-containing chemicals, as N-cyclohexyl-2benzothiazolesulphenamide (CBS) and dithiodianiline (DTDA), to achieve co-cure of MAH-EPM with other rubbers, which is absent with straight MAH-EPM.

Chapter 7 summaries three modification agents: CBS, 2,2'-dithiobis(pyridine-Noxide) (PNO) and caprolactam disulfide (CLD). The reactions of these modification agents with EPDM are described, respectively. Possible mechanisms are proposed as well. The results with one of this three justify further investigation.

Chapter 8 describes alkylphenol-polysulfide (APPS) grafted EPDM in blends with NR/BR for ozone-resistant tyre sidewall applications. The modification process, cure characteristics and significantly improved mechanical properties of the blends are emphasized.

Based on the results described in Chapter 8, mechanistic studies are presented in Chapter 9 to create a better understanding of the APPS grafting reaction with EPDM and the behaviour of APPS-grafted EPDM in blends with NR/BR. 
Finally, all the studies discussed in this thesis are summarised in Chapter10.

\section{REFERENCES}

1. White, J. L., in "Rubber Processing", Hanser Publishers, Munich Vienna New York, 1995.

2. Hess, W. M.; Herd, C. R.; Vegvari, P. C., Rubber Chem. Technol., 66, 329 (1993).

3. Zhao, J.; Ghebremeskel, G., Kautsch. Gummi Kunstst., 3, 84 (2001).

4. Chapman, A. V.; Tinker, A. J., Kautsch. Gummi Kunstst., 56, 533 (2003).

5. Ignatz-Hoover, F.; To, B. H.; Datta, R. N.; Hoog, A. J. d.; Huntink, N. M.; Talma, A. G., Rubber Chem. Technol., 76, 747 (2003).

6. Guo, R.; Talma, A. G.; Datta, R. N.; Dierkes, W. K.; Noordermeer, J. W. M., Eur. Pol. J., 44, 3890 (2008).

7. Waddell, W. H., Rubber Chem. Technol., 71, 590 (1998). 
Chapter 1 


\title{
Chapter 2
}

\section{Mixing, Curing and Reinforcement of Dissimilar Rubbers for Tyre Sidewall Applications}

\author{
A Literature Review
}

Tyre sidewalls generally consist of blends of natural rubber $(\mathrm{NR})$ and butadiene rubber (BR), containing a high concentration of antiozonants to provide ozone resistance. However, the most widely used antiozonant, $\mathrm{N}-(1, \quad 3$-dimethylbutyl)-N'-phenyl-pphenylenediamine (6PPD), is a staining, toxic and environmentally unfriendly substance. Incorporation of Ethylene-Propylene-Diene rubber (EPDM) into NR/BR is a way of achieving non-staining ozone resistance. But blending of dissimilar rubbers is severely restricted due to viscosity mismatch, thermodynamic incompatibility, cure incompatibility and heterogeneous filler distribution. This chapter gives an overview of the various research approaches in the field of blending dissimilar rubbers so far, as well as the mechanism of ozone protection by incorporation of EPDM in the tyre sidewall applications. 


\subsection{Introduction to Tyre Sidewalls}

Tyres have been around since the mid-1800s, and the earliest tyres were made of solid rubber. Until recently, tyres were available in a variety of different constructions, including bias-ply and bias-belted. Radial tyres have pretty much replaced other varieties since their technological design offers better safety and handling, particularly at highway-driving speeds. They enable better steering and more solid grip on the road, particularly when cornering or driving on curvy roads. They also last much longer than the older types of tyres.

The tyre sidewall is the outer surface of the tyre between the bead and the tread, as shown in figure 2.1. It provides a physical link between the wheel and the tyre tread in transmitting power and braking forces to the tyre tread. The tyre sidewall also plays a significant role in a vehicle's suspension and in the general handling of the vehicle on the road. As it undergoes distortion from the load bearing down on the tyre, one of the most significant properties of the sidewall is its flexibility ${ }^{1}$.

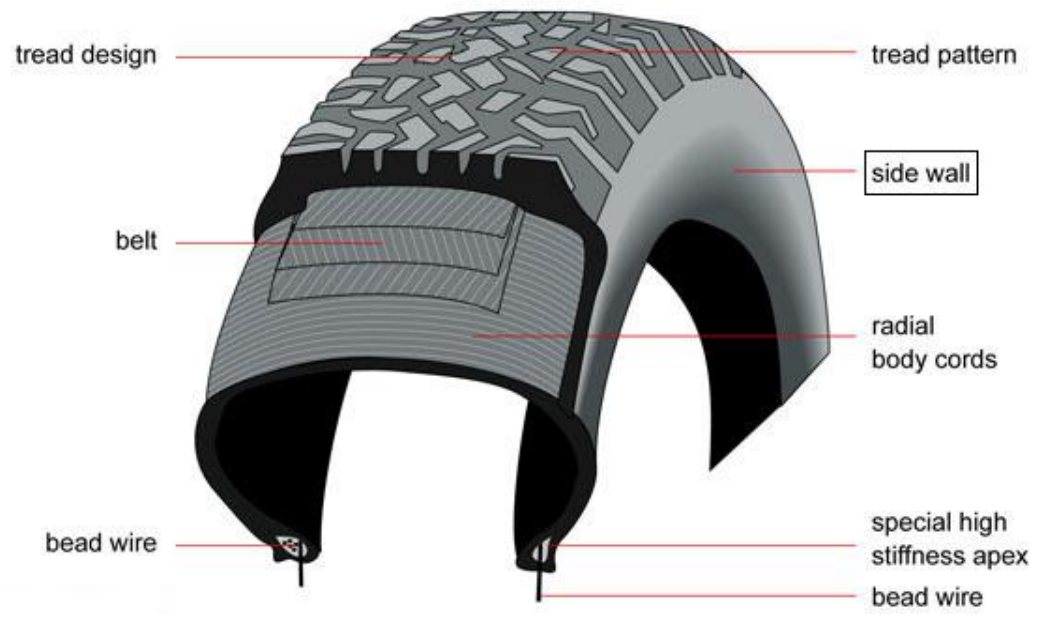

Figure 2.1 Various components of a radial tyre are shown in this cutaway view.

The sidewall consists of a set of casing plies covered with a thin layer of rubber. The tyre sidewall is thus the outer surface that protects the casing against weathering. It is formulated for resistance to weathering, ozone aging, abrasion, tearing and cracking, and for good fatigue life ${ }^{2}$. Typically, a sidewall compound contains a blend of natural rubber (NR) and butadiene rubber (BR) with carbon black as reinforcing agent and many other chemicals. Protection against ozone aging is of particular interest since the reaction of ozone with the olefinically unsaturated elastomers, as NR and BR, will result in polymer decomposition via chain scission (Fig. 2.2.). 


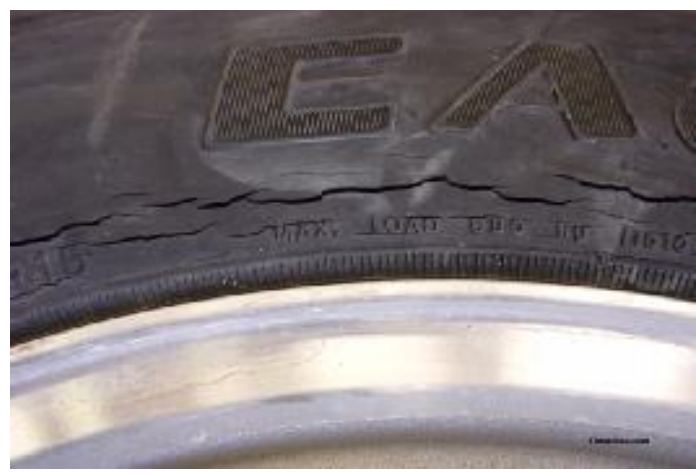

Figure 2.2 Sidewall with ozone cracks.

A tyre sidewall is a typical example where ozone protection is required under both static and dynamic conditions. It is standard practice in rubber compounding to use waxes and/or antiozonants in the formulation for effective ozone protection under both static and dynamic conditions.

Waxes exert their protection by blooming and forming a "physical" protective layer on the surface, which is impermeable to ozone. Wax offers effective protection in applications that are static or have a critical strain less than $30 \%$. The formation of the waxes film is based on the ability of wax to migrate to the surface. The governing factor for migration is its insolubility in the rubber matrix, which is a function of both the structure of the wax and temperature. Another important factor is the speed at which the protective film is formed. This depends on the solubility dependent migration and mobility of the molecules. Excessive migration or bloom occurs when the concentration of the wax greatly exceeds the solubility in the rubber. Blends of paraffinic and microcrystalline waxes are generally used to guarantee protection over the widest possible temperature range.

Classes of chemical antiozonants include: 6-alkoxy-2,2,4-trimethyl-1dihydroquinolines, $\mathrm{N}$-substituted thioureas, thiosemicarbazides, substituted pyrroles, nickel dithiocarbamate salts, 2,4,6-tris-(N-alkyl-para-phenylenediamino)-1,3,5triazines, triazinethiones and N,N'-disubstituted-para-phenylenediamines ${ }^{2}$. The $\mathrm{N}$ alkyl-N'-aryl-disubstituted para-phenylenedi-amines are the most effective class of antiozonants. They are easy handling, low-melting solids that are slowly destroyed by oxygen. N-1,3-dimethylbutyl,-N'-phenyl-para-phenylenedimine (6PPD) is the most widely used chemical antiozonant with the structure as shown in figure 2.3.

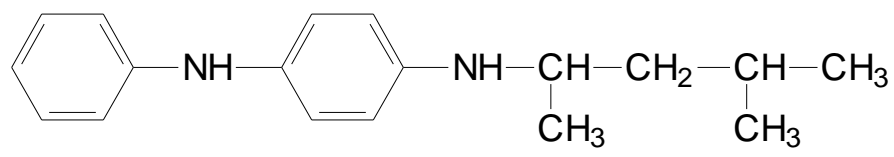

Figure 2.3 Structure of 6PPD. 
A number of mechanisms have been proposed to explain the functioning of antiozonants $^{1,2}$ :

I. Scavenger theory. The antiozonants diffuse to the rubber surface and react with ozone at a faster rate than ozone can react with the backbone carbon-carbon double bonds of unsaturated elastomers ${ }^{1-4}$;

II. Protective film theory. The reaction of ozone with the antiozonant produces a film on the surface of the rubber, which prevents ozone attack on the rubber and resistance to ozone permeation, like waxes are doing ${ }^{5}$;

III. Self-healing film theory. The reaction of antiozonant with the ozonized rubber forms a self-healing film on the rubber surface ${ }^{6}$;

IV. Relinking model. The antiozonant migrates to the ozone cleaved polymer chains and reacts with the carbonyl and/or carboxy end groups to recouple these ends into a patched chain ${ }^{7}$.

However, the addition of antiozonants has some inherent disadvantages. Firstly, the protection by an antiozonant of the rubber once it is mixed into the compound, does not last forever. The antiozonant is continually depleted from the tyre sidewall surface by reaction with ozone and by physical mechanisms such as curb scuffing and washing. One can easily imagine that as a result of this phenomenon, there will be a certain moment that the concentration of antiozonants is too low to provide the necessary protection.

Secondly, para-phenylene diamines (PPDs) are highly toxic materials. Therefore, from an environmental point of view it is worth to search for alternatives.

The major disadvantage of PPDs is their staining characteristic, which renders them unsuitable for light colored applications. Black sidewall surface discoloration by the formation of a thin brown film of ozone/PPD reaction product is unacceptable to many customers as well.

New developments on antiozonants have focused on non-staining and slowmigrating products which last longer in rubber compounds. Several new types of nonstaining antiozonants have been developed, but none of them appeared to be as efficient as the conventional antiozonants. The most prevalent method to achieve non-staining ozone protection of diene rubbers is to blend them with inherently ozone-resistant, saturated backbone polymers ${ }^{8}$ like EPDM.

\subsection{Blending of Dissimilar Rubbers}

There is an increased technological interest in the use of blends of various dissimilar rubbers to achieve tunable enhanced properties. But blending of dissimilar rubbers is severely restricted due to lack of miscibility and technological compatibility of the component rubbers. Very often it happens that the components are grossly 
immiscible as well as technologically incompatible. Four types of mutual incompatibility can generally be discerned between dissimilar elastomers: imcompatibility due to viscosity mismatch which prevents or greatly delays the formation of intimate blends ${ }^{9}$; thermodynamic incompatibility, which prevents mixing on a molecular scale; imcompatibility due to cure rate mismatch ${ }^{10}$; and uneven reinforcing filler distribution. The latter two are two most important aspects in rubber blends, as will be discussed in the following sections.

\subsubsection{Viscosity Mismatch}

There is a relation between the mixing viscosities of the component rubbers and the morphology of the resulting blends. The relative mixing viscosities of the components affect the size and the shape of the domain zones.

Avgeropoulos $^{9}$ studied the relation between rheology and morphology of EPDM/BR blends. He changed the blend morphology by adjusting the mixing torque or mixing temperature. In this way he found that for a given composition, the smallest size domains were obtained when the mixing viscosities of the components were equal. While, for a blend system in which the mixing viscosities of the components were not equal, the lower viscosity component encapsulated the higher viscosity component and became the continuous phase.

Therefore, if incompatibility is only encountered because of viscosity mismatch, one can greatly improve the blending process and in some cases also the quality of the blend by adjusting extender oil and filler concentration in the dissimilar elastomers and so adjusting the relative viscosities of the individual elastomers simply by changing the shear rate and/or mixing temperature or milling time.

Chang ${ }^{11}$ used trans-Polyoctylene rubber (TOR) as a potential compatibilizer for NR/EPDM blends and found that TOR acts as a processing aid and causes a reduction in melt viscosity in the NR/EPDM blend. A Fine dispersion of EPDM particles in the NR matrix was achieved by the addition of TOR. As a result of improved compatibility, ozone resistance and dynamic properties of the NR/EPDM blend significantly increased.

\subsubsection{Thermodynamic Incompatibility}

Complete miscibility on a molecular scale does not occur for blends of most polymers because of unfavourable free energies of mixing. Greater miscibility is favoured for polymers containing polar groups [e.g. Polyvinylchloride (PVC), Nitriel rubber (NBR)]. For non-polar polymers, miscibility occurs only when their solubility parameters closely match.

The solubility parameter $\delta$ of non-electrolytes can be determined from their cohesive energy density (C.E.D) and consequently their heat of vaporization ${ }^{12}$ : 


$$
\delta=(\text { C.E.D. })^{1 / 2}=\left[\frac{H_{v}-R T}{V}\right]^{1 / 2}
$$

where, $H_{v}$ is the molar heat of vaporization, $R$ is the gas constant, $T$ the absolute temperature and $\mathrm{V}$ is the molar volume. Thus, the solubility parameters of low molecular compounds can be experimentally measured by determining the heat of vaporization. The solubility parameters of some typical elastomers, as determined by their solubility in low molecular weight liquids of known solubility parameters, are given in Table 2.1.

Table 2.1 Some experimental values of solubility parameters ${ }^{13,14}$.

\begin{tabular}{cc}
\hline Polymer & Solubility parameter $\delta\left[\left(\mathrm{J} / \mathrm{cm}^{3}\right)^{1 / 2}\right]$ \\
\hline 1,4- Cis BR & 17.14 \\
BR & 16.99 \\
NR & 16.73 \\
EPDM & 15.95 \\
SBR (40\% Styrene) & 17.87 \\
SBR (23\% Styrene) & 17.46 \\
\hline
\end{tabular}

Hildebrand and Scott ${ }^{12}$ had also developed a relationship, between the enthalpy of mixing of two liquids: $\Delta \mathrm{H}_{\mathrm{m}}$, and their solubility parameters $\delta_{1}$ and $\delta_{2}$. This is given by the following equation:

$$
\Delta H_{m} / V_{1}=K\left(\delta_{1}-\delta_{2}\right)^{2} \Phi_{1} \Phi_{2}
$$

where, $\mathrm{V}_{1}$ is the average molar volume of the two liquids, $\mathrm{K}$ is a constant close to $1, \delta_{1}, \Phi_{1}$ and $\delta_{2}, \Phi_{2}$ are the solubility parameters and volume fractions of components 1 and 2 , respectively.

From this equation it can be seen that blends of polymers with large differences in $\delta$ require more energy for dispersion. Therefore, for a pair of polymers, if the difference in solubility parameters is small enough $\left[\leq 0.1\left(\mathrm{~J} / \mathrm{cm}^{3}\right)^{1 / 2}\right]$, there may be a potential for molecular miscibility ${ }^{15}$.

If only thermodynamic incompatibility is encountered, good blend properties can be achieved by reducing the interfacial tension between the elastomer blend constituents with additives like compatibilizers; or by minimizing the surface energy of the elastomer constituents so as to permit the formation of very small microdomains 
of the individual elastomer phases; or by developing sufficient adhesion between the phases, e.g. by the formation of crosslinks across the interface during vulcanisation.

Mixtures of high-diene rubbers with combinations of EPDM and halobutyl rubber have been proposed for use in tyre sidewalls which are resistant to ozoneinduced flex-cracking ${ }^{16}$.

Polar polymers as polychloroprene ${ }^{17}$, chlorinated polyethylene, and chlorosulphonated polyethylene ${ }^{18}$ have been employed as the third polymer components in EPDM/NBR blends. The better perfomance of these polar polymers as compatibilizing agents has been attributed to their affinity towards the highly polar NBR component ${ }^{17}$.

A usual procedure to improve the interfacial adhesion and mechanical properties of heterogeneous polymer blends consists of the addition of block or graft copolymers whose individual segments are identical or miscible with the respective blend components. Provided that the block lengths are sufficiently long, the additive will preferentially be located at the interface in a configuration whereby it is intimately mingled with each phase ${ }^{15}$. Zanzig et $\mathrm{al}^{19}$ have reported the use of block copolymers of polyisoprene and polybutadiene to compatibilize blends of cured and uncured NR and BR blends.

But for this procedure, a well-controlled polymerisation procedure is required to prepare these block- and graft-copolymers with specific structure. Therefore, a process, namely in situ compatibilization or reactive compatibilization was developed, where these block- and graft-copolymers can be formed in situ, during blending, by using appropriate functional polymers, which are able to react with the other blend component under the blending conditions. In Oliveira's work, improved mechanical properties could be achieved by using mercapto-modified EPDM as compatibilizing agent in NR/EPDM and NBR/EPDM blends ${ }^{20}$.

Hydrogen bonding interaction in modified polymers can also lead to compatibilization. Ismail and co-workers ${ }^{21}$ found that the presence of hydrogen bonding in PVC/Acrylic modified NBR blends improved the mechanical properties of the blends.

\subsection{Curing of Dissimilar Rubbers}

With respect to elastomer-elastomer blends, the most essential crosslinking characteristic is the ability of the two elastomers to covulcanise. Covulcanisation is a term which should be applied to the formation of a single network structure, including crosslinked macromolecules of the two elastomers. That is to say both elastomers should become vulcanised to similar extents with crosslinking between different elastomers, across microdomain interphases. 
Cure incompatibility becomes an issue when the elastomers are both vulcanised by the action of the same curing ingredients but at reaction rates and scorch times which are significantly different for each of the elastomers. Reactivity differences between the elastomers and differences between the solubilities of curatives in the elastomers contribute to this incompatibility ${ }^{22-25}$.

It has been known for long that the ozone resistance of high-diene rubbers such as NR, SBR, NBR, BR can be greatly improved by the incorporation therein of lowunsaturation rubbers, such as EPDM. However, the vulcanisates of such elastomer blends are generally poor in both static and dynamic mechanical properties: ultimate strength-related properties, fatigue resistance, hysteresis, etc.. The observed inverse relationship between the increasing ozone resistance and the loss of general physical properties of EPDM/high-diene blends can be explained by mainly the cure imcompatibility.

The latest developments to overcome this problem can be grouped into several basic approaches:

A. Increase the solubility of curatives in the EPDM phase relative to the high-diene matrix. This affects both the co-curability of the different phases and promotes interfacial interaction between the different phases.

B. Modify EPDM by grafting accelerators or accelerator precursors onto it. This will propagate vulcanisation outward from the EPDM into the highdiene matrix.

C. Use a different mixing procedure to improve mechanical properties of EPDM/high-diene blends.

D. Consider the creation of another adhesion mechanism which does not rely on interphase cross-links between the EPDM and the high-diene matrix, e.g. block co-polymer entanglement or ionomeric functionalisation.

\subsubsection{Diffusion and Solubility of Curatives}

The driving force for the diffusion of curatives in dissimilar elastomer blends is the absence of equilibrium caused by the difference in concentrations of the diffusates ( $\mathrm{ZnO}$, sulphur and accelerators), coupled with the tendency to eliminate this difference by molecular motion. It also depends on the difference in solubility of the diffusates between the dissimilar elastomers.

Diffusion in an isotropic substance is based on the assumption that the rate of transfer, $\mathrm{R}$, of the diffusing matter through a unit area is proportional to the concentration gradient: 


$$
\begin{aligned}
& R=-D \frac{\partial c}{\partial x} \quad \text { (Fick's first law) } \\
& \text { And } \quad q=-D A \int_{0}^{t}\left(\frac{d c}{d x}\right) d t
\end{aligned}
$$

Where $\mathrm{D}$ is the diffusion coefficient; $\mathrm{c}$ is the concentration of diffusing matter; $\mathrm{x}$ is the space coordinate measured normal to the cross section; and $q$ is the amount of diffusing substance passing a cross section of surface area $A$, in total time t. The diffusion coefficient is given by equation (eq. 2.5):

$$
D=\frac{Q}{S}
$$

Where $Q$ is the permeation coefficient and $S$ is the solubility coefficient. In blends of EPDM with high-diene rubbers, not only the curative diffusion depends on the concentration gradient of curatives between EPDM and high-diene rubbers, but also the differences in scorch time, cure rate, and curative solubilities between those polymers.

Curative diffusion between the domains of an elastomer blend takes place during vulcanisation. This process may deplete curatives from one side of the polymer-polymer interface and speed up cure on the other side. Thus, there is an interfacial layer of rubber with a different state of cure than the bulk, resulting in a weaker layer of rubber at the interface which may reduce adhesion ${ }^{26}$.

Gardiner ${ }^{27-29}$ studied curative diffusion across the boundaries of several different polymer combinations. The individual polymers were chlorobutyl rubber (CIIR), butyl rubber (IIR), EPDM, chloroprene rubber (CR), SBR, BR and NR. The results showed curatives diffusion from the less polar to the more polar elastomer phase. This diffusion was shown to occur very quickly during both the mixing and the vulcanisation phases of compound processing. Gardiner further noted that the very polar thiuram disulphide accelerators showed the greatest tendency to migrate because of their much greater solubility in the polar elastomer phase of a blend.

Table 2.2 summarized the solubilities of three normally used curatives: sulphur, mercaptobenzothiazole (MBT), and tetramethylthiuram disulphide (TMTD) in three elastomers: SBR, EPDM, and $\mathrm{BR}$ and their blends at $153^{\circ} \mathrm{C}$ which is the vulcanisation temperature ${ }^{30}$. It can be seen that the three ingredients are more soluble in SBR than in the other two elastomers. The differences are large enough to matter, as shown by the solubility ratios on the right side of Table 2.2. Although it is 
not so obvious as compared to SBR, the three curatives still show higher solubility in BR than in EPDM. Therefore, polymer unsaturation plays a role in curative migration.

Table 2.2 Solubilities of curatives in different elastomers and blends at $153^{\circ} \mathrm{C}$.

\begin{tabular}{c|ccc|ccc}
\hline & \multicolumn{3}{|c|}{ Solubility at $153^{\circ} \mathrm{C}$, phr } & \multicolumn{3}{c}{ Ratio of solubilities } \\
\cline { 2 - 7 } & SBR & EPDM & BR & SBR/EPDM & SBR/BR & BR/EPDM \\
\hline S & 17.3 & 10.7 & 16.8 & 1.62 & 1.03 & 1.57 \\
MBT & 5.2 & 1.1 & 2.4 & 4.65 & 2.16 & 1.92 \\
TMTD & 14.3 & 5 & 4.9 & 2.86 & 2.92 & 0.98 \\
\hline
\end{tabular}

Brown and Tinker $^{31}$ have presented evidence that in the NR/EPDM system they studied, there was considerable diffusion of crosslinking agents from the EPDM to the natural rubber, resulting in a high crosslink density of the NR phase.

Zhao $^{22}$ investigated EPDM/SBR blends and found that with a sulphur cure system, optimum properties of EPDM/SBR blends were achieved by selecting accelerators, which have high solubility in the EPDM phase and give rise to a shorter scorch time and faster initial cure rate of the EPDM phase. Thus, EPDM/SBR blends cured with sulphenamide accelerator were found to have better curing performance and improved mechanical properties as compared to thiuram and thiazole accelerators.

In order to achieve covulcanised properties in NBR/EPDM blends, Woods and Davidson $^{32}$ used less polar accelerators which were prepared by increasing the length of the alkyl group in zinc dialkyldithiocarbamate accelerator molecules. They found that there was a gradual improvement in blend properties as the length of the alkyl group increased. They also found a surprising result that covulcanisation could be obtained in NBR/EPDM blends with polar tetraethylthiuram disulphide (TETD) accelerator formulations when all or part of the zinc oxide used in their first study was replaced with lead oxide. The reason for that result is that the lead salts of TETD are insoluble in both polar and nonpolar materials. This insolubility would remove the thermodynamic driving force for curative diffusion between elastomer phases and result in a uniform concentration of accelerator salt in both phases. The resulting blends would then be well vulcanised in both phases and exhibit good properties. These observations are consistent with the hypothesis that lack of covulcanisation is due to the diffusion of the accelerator into the more polar and faster curing phase of an elastomer blend.

Shershnev $^{33}$ has summarized the various means of achieving good covulcanisation in blends of high and low unsaturation elastomers in terms of:

1. Separate masterbatches with varied curative loading;

2. Accelerators with a high degree of alkylation; 
3. Use of ingredients that form insoluble compounds after reacting with accelerators and other vulcanising agents;

4. Use of vulcanising agents which distribute uniformly and have similar activities for different elastomers, e.g. peroxides and reactive resins.

\subsubsection{Elastomer Modification}

The main problems associated with the generally poor properties of EPDM/high-diene blends are large relative differences in the chemical vulcanisation reactivity of EPDM and high-diene rubbers. Such a disparity in reactivity is reflected in the relative rates of crosslinking during vulcanisation, both within and between the two phases, diffusion of curatives towards the faster curing high-diene phase occurring readily during mixing and curing.

There have been several approaches to improve the properties of EPDM/highdiene rubber blends. Generally, these approaches have sought to increase the cure rate of EPDM, either by using curatives that have an increased reactivity towards EPDM, which has been discussed in the previous section, or by means of modifying the EPDM to make it more reactive towards curatives.

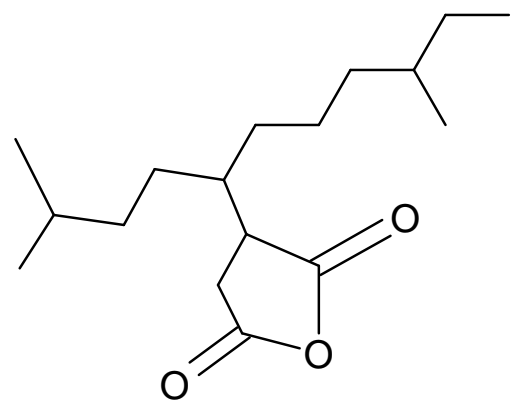

Figure 2.4 Schematic representation of EPDM-MA.

Coran $^{34}$ achieved better cure compatibility for EPDM/NR blends by modifying the EPDM with maleic anhydride: Figure. 2.4. This permits the EPDM to be crosslinked independently with the zinc oxide in the accelerated-sulphur vulcanising system. An ionic crosslink network is produced in the EPDM phase. This type of crosslinking is not competitive with the accelerated sulphur system, which reacts rapidly with the NR. Compared to conventional NR/EPDM blends, those with the modified EPDM exhibited higher tensile properties and fatigue life along with reduced hysteresis and permanent set, all of which reflect better covulcanisation.

In Oliveira and Soares's work, they studied the functionalisation of EPDM with mercapto groups by a simple free radical addition reaction and found that this mercapto-modified EPDM: Figure. 2.5, can be used to improve the mechanical properties of EPDM-based elastomer blends ${ }^{35}$. 


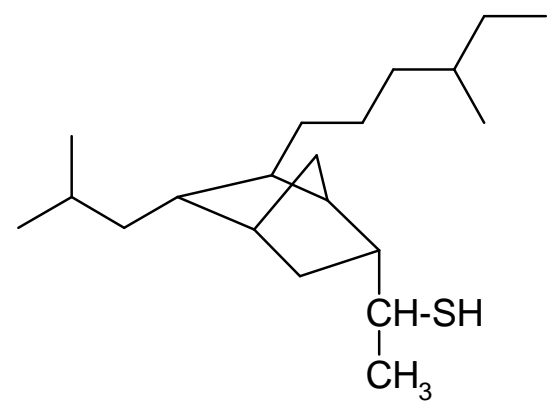

Figure 2.5 Schematic representation of mercapto-functionalised EPDM.

Hopper $^{36}$ modified hexadiene EPDM with $\mathrm{N}$-chlorothioamides in solution to give pendent groups which would act in the manner of a retarder: Fig. 2.6, to high-diene vulcanisation. Blends of the modified EPDM with synthetic polyisoprene (IR) exhibited higher modulus and tensile strength together with lower heat build-up, and this was taken as evidence of improved vulcanisation of EPDM. Hopper proposed mechanisms by which the pendent groups could result in the formation of additional crosslinks in the EPDM. As EPDM modification leading to pendent thio- $\mathrm{N}$-methyl-ptoluenesulphonamide group, it was found that 2-mercaptobenzothiazole (MBT) displaced the N-methyl-p-toluenesulphonamide to form a crosslink precursor, which then participates in the normal vulcanisation reactions to form either a crosslink with EPDM or NR.

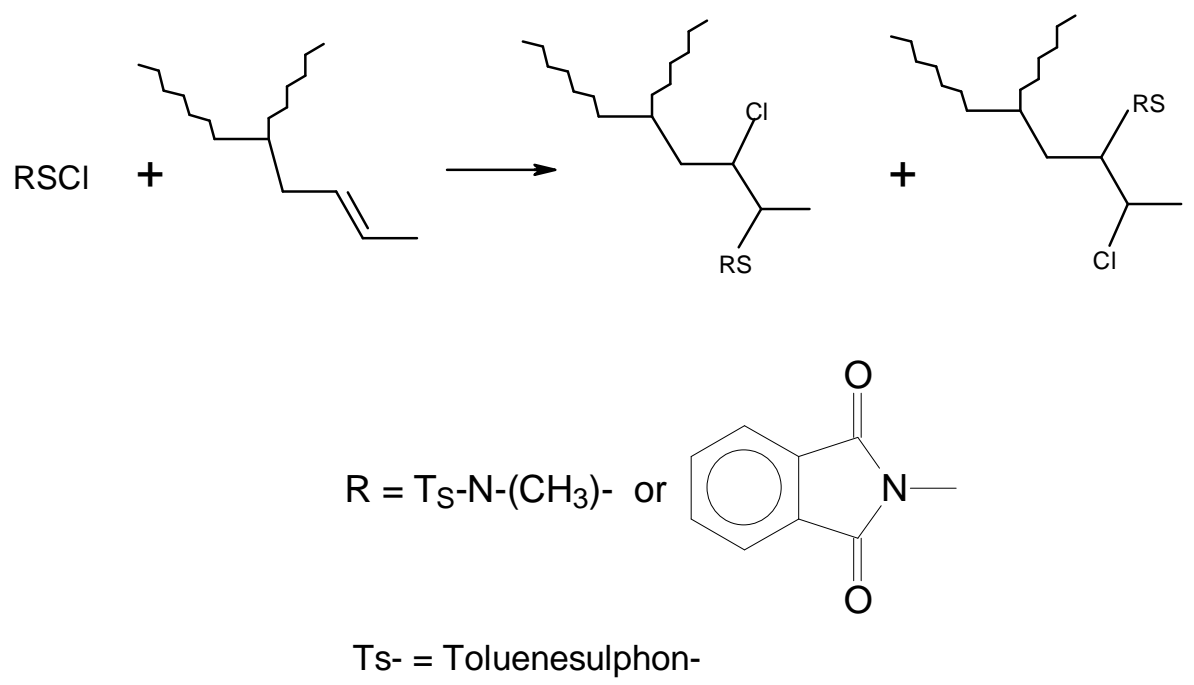

Figure 2.6 Schematic representation of N-chlorothioamide-functionalized hexadiene-EPDM.

Baranwal and Son ${ }^{37}$ described a method of achieving co-cure by grafting an accelerator onto EPDM. They modified EPDM in solution with accelerator species, including the sulphur donor dithiodimorpholine (DTDM), using UV irradiation in the 
presence of a photosensitiser. The result showed that by grafting accelerators onto EPDM, excellent cure compatibility could be obtained.

Morrissey ${ }^{38}$ reported that halogenation of EPDM in solution could result in a marked improvement in their cure compatibility with the highly unsaturated rubbers.

All of the EPDM modification methods described above achieve a significant improvement in the physical properties of EPDM/high-diene blends. This results directly from the introduction of reactive sites able to participate in the cure process and to speed up vulcanisation in the EPDM phase, therefore improving the level of crosslinking achieved.

\subsubsection{Mixing and Precuring Procedure}

\subsubsection{Reactive mixing}

$\mathrm{Cook}^{39}$ maded a similar approach as the one developed in the work of Hopper ${ }^{36}$ and Baranwal and Son ${ }^{37}$. Three commercially available sulphur donors, bisalkylphenoldisulphide (BAPD), dithiodicaprolactam (DTDC) and dithiodimorpholine (DTDM) were used to modify EPDM by mixing at elevated temperatures in an internal mixer as part of a normal masterbatch mixing cycle. The procedure is called 'reactive mixing'. According to $\mathrm{Cook}^{39}$ and Hopper ${ }^{36}$, a certain functionality: alkylphenolmonosulphide, caprolactam or morpholino coming from the sulphur donor is attached to the EPDM via a sulphur linkage. During vulcanisation, all of these groups can act as leaving groups, to be substituted by MBT that is known as a vulcanisation intermediate. In this way a crosslink precursor site on the EPDM polymer chain is formed. A simplified representation of the reaction mechanism is shown in Figure 2.7.

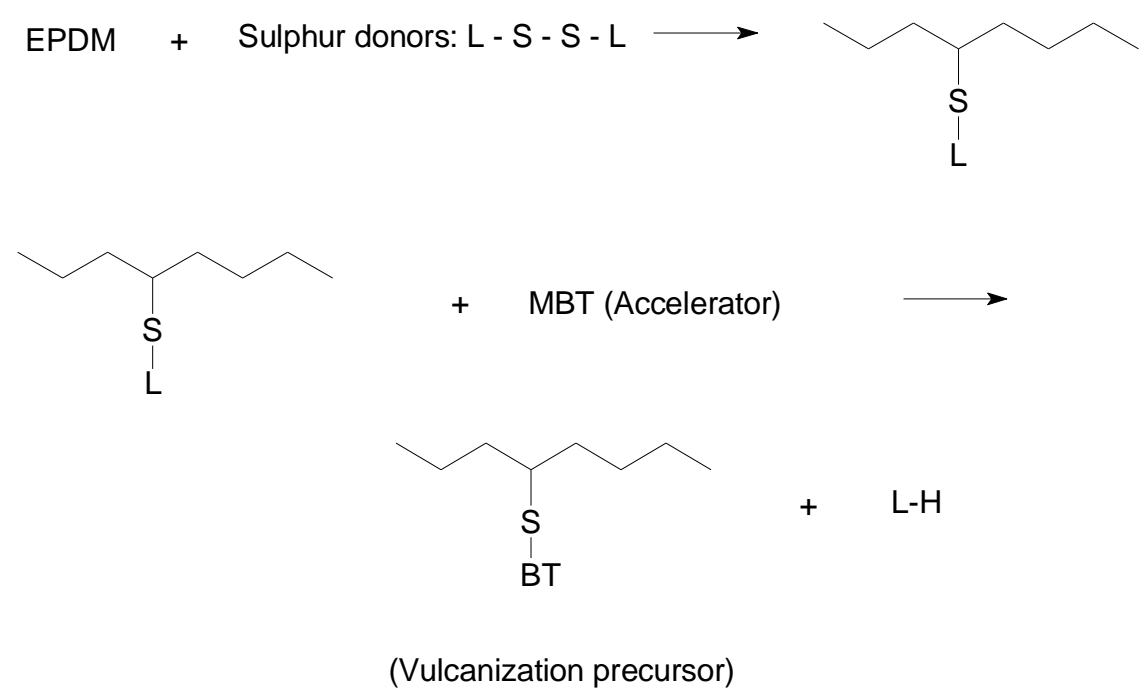

Figure 2.7 Reaction mechanism of EPDM modification and formation of vulcanisation precursor: $\mathrm{L}=$ alkylphenolmonosulphide, caprolactam or morpholine. 
The properties of these modified-EPDM/NR blends were in general better than the control blend without modification of the EPDM phase. With the same amount of curatives, an increase of crosslink density was achieved, which was sufficient to bring about considerable improvements in tensile strength. Meanwhile, it was seen that modification of EPDM by BAPD lead to significantly smaller blend phase size than in the unmodified EPDM blend. Modification with DTDC and DTDM appeared to have less effect on phase size.

\subsubsection{Precuring}

Precuring has also been used to improve the mechanical properties of NR/EPDM blends ${ }^{40}$. The precuring in the EPDM possibly reduces the migration of the curing agents out of EPDM into the NR phase. In this way, it was tried to overcome the undercuring of the EPDM. This undercuring of the EPDM in NR/EPDM blends is the result of the lower cure rate that is caused by the lower unsaturation and lower solubility of the curatives in EPDM relative to NR. In Suma's work ${ }^{40}$, a low degree of precuring of the EPDM phase in NR/EPDM blends helped to attain a covulcanised state in these blends after the final curing. Mechanical properties, which are influenced by the crosslink densities in both the phases and in the interface, are remarkably improved by the precuring.

Ghosh and $\mathrm{Basu}^{41}$ have observed that NR and SBR could be effectively covulcanised with EPDM in presence of a multi-functional additive like bis(diisopropyl) thiophosphoryl disulphide (DIPDIS) using a new vulcanisation technique, which is two-stage vulcanisation. In that procedure, high-diene rubber and EPDM were first masticated separately. The whole amount of sulphur, accelerator and other additives were incorporated in EPDM. The compounded EPDM mix was then heated to obtain a grossly undercured mix (modified EPDM). In the end, masticated high-diene rubber and modified EPDM were mixed together to prepare the final compounds. Compared to one-stage vulcanisation, precuring of EPDM was introduced into two-stage vulcanisation as well.

Sahakaro ${ }^{42-44}$ et al. also used that method to prepare NR/BR/EPDM blends. The entyre amount of curatives was first added into the EPDM phase. After a thermal pretreatment step, the modified EPDM was mixed with pre-masticated NR/BR. The reactive blend vulcanisates showed a significant improvement in tensile properties as compared to those prepared by straight mixing.

\subsubsection{Other Approaches}

In Brodsky's work ${ }^{45}$, improved covulcanisation could be achieved by using a tricure system which was based on accelerator, sulphur and a peroxide. The tri-cure system was believed to minimize the cure imbalance and reversion faced with 
conventional cures. The results showed improved cut growth and dynamic ozone resistance. The tri-cure system brings the EPDM/NR/BR sidewall another step closer to achieving the performance of the NR/BR sidewall benchmark.

Zhao and Ghebremeskel's ${ }^{46}$ study also showed that the addition of a small amount of sulphur as a coagent to a peroxide cure system in EPDM/SBR compounds has a remarkable positive influence on all the mechanical properties.

In the case of EPDM systems, some people also employ a binary accelerator system containing thiuram as one of the accelerator components. The presence of lead dithiocarbamates, formed in or incorporated into the EPDM before its admixture with NBR gave significant improvements with respect to the ultimate properties of the blends $^{32}$. Also, an increase in the level of unsaturation in the EPDM molecules gives rise to improvements in properties of its blends with high-diene rubbers.

\subsection{Reinforcement of Dissimilar Rubbers}

The incorporation of fillers, such as carbon black, into a rubber is of significant importance, since fillers not only enhances the mechanical properties of the final products but also decrease the cost of the end products. For practical applications of reinforced rubber blends, the phase morphology as well as crosslinker and filler distributions and dispersions in each individual phase all play an important role in determining the physical properties of the blends.

\subsubsection{Rubber Reinforcement}

Elastomers, in general, are not used in their pure form, but are reinforced by fillers. The dramatic increase in properties like modulus, hardness, tear strength, and abrasion resistance that occurs after filler addition has caused many researchers to find the reason for the reinforcement. Carbon black and precipitated silica are the most well-known reinforcing fillers for elastomers.

Figure 2.8 shows the strain-dependence of the complex modulus: the Payne effect, and the strain-independent contributions to the modulus for carbon black filled compounds and silica filled compounds. The contributions to the complex modulus are the filler-filler interaction, filler-polymer interaction, the polymer network contribution and the hydrodynamic effect due to the presence of the solid filler particles in the visco-elastic matrix. 


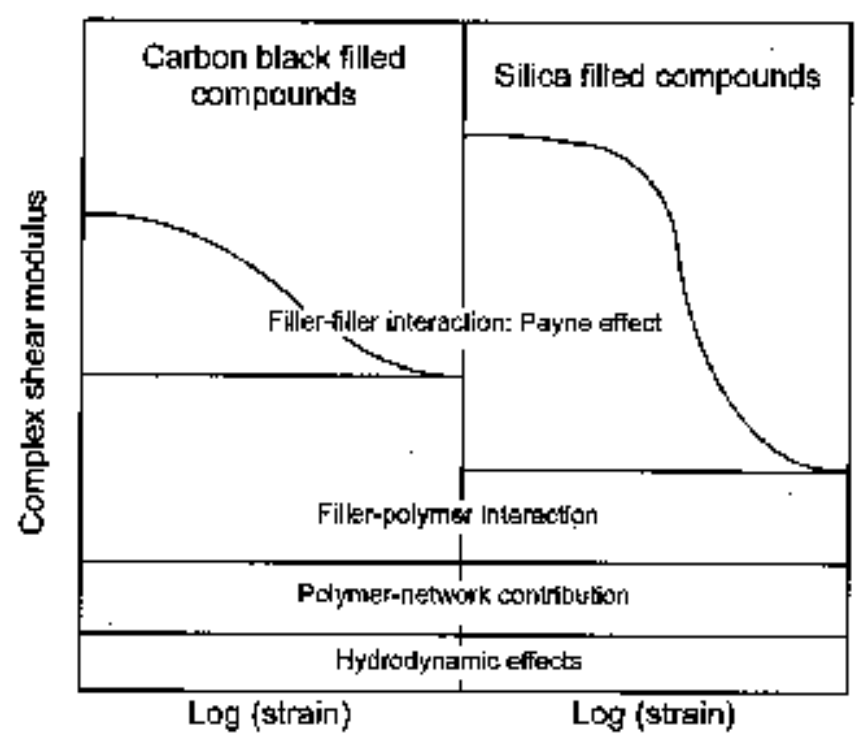

Figure 2.8 Effects contributing to the complex modulus

The reinforcement is dependent on the interaction between the filler particles and the polymer. These interactions can be strong, for example in the case of covalent bonds between functional groups on the filler surface and the polymer, or weak as in the case of physical attractive forces. When carbon black is blended with a polymer, the level of physical interaction is high. In contrast to this, the interaction between silica particles and polymer is very weak, and only by the use of a coupling agent a chemical bond is formed between the filler and the polymer ${ }^{47}$. The occluded rubber contributes to this interaction: Polymer chains are trapped in the voids of the filler aggregates; they are immobilized and shielded from deformation. They do not contribute to the elastic behaviour of the matrix, as their properties resemble the properties of the rigid filler particles rather than the properties of the elastic and flexible free polymer chains. Occluded rubber increases the effective filler loading and thus the strain independent contribution to the modulus.

Bound rubber has been recognized as an important factor in the mechanism of rubber reinforcement. The filler-polymer interaction affects the level of bound rubber content as well. The interaction leading to the formation of bound rubber involves physical adsorption, chemisorption, and mechanical interaction ${ }^{48}$.

Besides the interaction between the polymer and the filler, an interaction between filler particles themselves also occurs. The filler-filler interactions most prominently influence the material characteristics by the Payne effect. This straindependent contribution to the modulus is caused by the reinforcing filler-filler interactions. From figure 2.8, it can be seen that the elasticity modulus of reinforced rubber depends on the magnitude of the deformation. On increasing strains, the modulus decreases. This effect was first described by Payne, and he interpreted the 
sigmoidal decrease of the storage modulus versus the deformation amplitude by such a model that the filler forms its own co-continuous network inside the rubber network and, on deformation, this filler network is disturbed as the result of the breakage of physical bonds between filler particles. This effect is largely reversible once the strain is released and is independent of the type of polymer, but is dependent on the type of filler. Figure 2.8 also shows the key difference between carbon black and silica: The Payne-effect is stronger for silica, as a consequence of the stronger polar interparticle forces between the filler particles ${ }^{49}$.

\subsubsection{Distribution of Carbon Black}

Filler distributions and dispersions in each individual phase play an important role in determining the physical properties of the blends. The affinity of carbon black for a given polymer in each individual phase depends on factors such as the degree of unsaturation, the polarity, the viscosity, and the molecular weight of the polymers, as well as the mixing conditions and carbon black characteristics ${ }^{50}$.

\subsubsection{Effect of rubber properties}

For common commercial elastomers, the affinity of carbon black for polymers was reported to have the following decreasing order: $\mathrm{BR}>\mathrm{SBR}>$ polyisoprene $>\mathrm{NR}$ $>$ EPDM > butyl rubber ${ }^{51}$.

Klüppel and coworkers ${ }^{13}$ studied the carbon black distribution in rubber blends and found that carbon black (N550) in BR/EPDM blends is preferably located in the BR phase. They postulated that this is related to the high affinity between the free electrons in carbon black and the BR double bonds. In EPDM, double bonds are rare, causing a poor compatibility between EPDM and carbon black.

For blends such as SBR/BR, the concentration of carbon black in one phase was found to increase with increasing molecular weight of the polymer provided that the molecular weight of the second polymer remains the same. This can be explained by measuring bound rubber which increases with increasing molecular weight: higher molecular weights are preferentially adsorbed on the filler surface for entropic reasons. ${ }^{52}$

\subsubsection{Effect of mixing conditions}

Various ways of incorporating carbon black have been used to prepare polymer blends. Both mechnical and solution blending methods have been used. Typical methods are:

1) Mixing two polymers and carbon black in one operation; 
2) Mixing the first polymer with carbon black and then diluting with the second polymer;

3) Preblending polymers and then mixing with carbon black;

4) Mixing black masterbatches of the first polymer and of the second polymer, and then blending the two masterbatches.

Uneven distribution of carbon black in the two polymer phases is often observed, the extent of which depends on the mixing method. And it was found that carbon black can migrate from one polymer which has a lower affinity towards carbon black, to the other having a greater affinity even when carbon black was first incorporated in the individual polymers ${ }^{51}$.

In the study of Van de Ven and Noordermeer ${ }^{53}$, they found that the influence of the carbon black distribution was much more important than any other aspect in blends of EPDM and NR/BR. This was the result of using two different mixing techniques: straight mixing and masterbatch mixing. A masterbatch mixing technique accounted for a better dispersion of the filler than does straight mixing. The results showed that the masterbatch mixing technique improved the properties much more than using an EPDM type with higher ENB-level or molecular weight. Surprisingly, the blend based on the EPDM type which according to earlier studies was assumed to perform the best (high ENB-level and high molecular weight), showed the worst properties of all. As a matter of fact, a very common EPDM type (medium ENB level and low Mooney) nearly matched the performance of the reference NR/BR blend compound. This strengthens the fact that people tend to underestimate the importance of carbon black distribution between the different rubber phases in the blend.

Herd and Bomo ${ }^{54}$ used different mixing methods to control the carbon black distribution and studied the effects on NR/EPDM rubber properties such as modulus, failure properties, hysteresis and processability characteristics. The best balance of tyre sidewall properties was obtained in the preblend in which all of the carbon black was preferentially located in the NR, and in the blend prepared with a NR and EPDM masterbatch, in which half the carbon black was in the NR phase and the other half in the EPDM phase. The latter blend did have significantly lower hysteresis which is more favourable in sidewall applications.

The tendency of migration of carbon black was reported to depend on the mechanical and thermal history used in preparing the masterbatches, and conditions and time of blending ${ }^{26}$ as well. There was no indication of significant transfer to BR or SBR from typical carbon black-NR Banbury mixes with high heat history. However, transfer from NR solution and latex masterbatches, as well as low temperature mill mixes, did take place. Extensive carbon black transfer from a low unsaturation 
elastomer (IIR) to other high unsaturation polymers was found regardless of the masterbatch mixing procedure.

The phenomena observed in the various studies are often so conflicting, as they depend on the particular systems investigated, that it is hard to draw clear conclusions.

\subsubsection{Effect of characteristics of carbon black}

The characteristics of carbon black such as specific surface area, structure and chemical surface functionalities also influence its distribution in a polymer blend.

For NR/SBR blends it was found that, structure as measured by DBP (dibutylphtalete) adsorption, oxidation and heat treatment of carbon black in inert atmosphere at $1500^{\circ} \mathrm{C}$ (graphitisation) had little effect on the distribution of the black. However, the distribution of carbon black was found to be sensitive to the specific CTAB (cetyl trimethyl ammonium bromide) surface area: the concentration of carbon black in NR increased linearly with this surface area ${ }^{55}$.

A standard and partially graphitized N650 type carbon black were employed to study transfer in NR/EPDM blends. After graphitisation, carbon blacks are reduced to a minimum level of surface activity and then behave as an inert filler in terms of polymer interaction. In this study, the untreated N650 sample located almost entyrely in the NR phase when added to the preblend. On the other hand, the partially graphitized carbon black was distributed in both polymers with some preference for the EPDM. These results indicate that for N650, the carbon black/elastomer interaction in the unsaturated NR phase is playing a dominant role in controlling the phase distribution. For partially graphitized N650, it appears that molecular weight or viscosity plays more of a dominant role in determining the location of the carbon black $^{26,54}$.

The above studies confirm the fact that carbon black transfer is a complex phenomenon. A promising technique to direct the carbon black into one phase and force it to remain in that phase is the dynamic vulcanisation approach. In this process carbon black and curatives are mixed together into one component to become the dispersed phase in a blend. That curative containing compound is then mixed in a non-productive stage into the other component to become the continuous phase, and some crosslinking occurs which "blocks" the filler in the desired phase ${ }^{50}$.

\subsubsection{Silica}

In the tyre industry, especially nowadays there is a strong increase in the use of silica in tyres, because precipitated silica in combination with S-SBR offers the possibility to reduce the rolling resistance with $30 \%$, which results in fuel saving. This 
is achieved because of a lower hysteresis at $60^{\circ} \mathrm{C}$, while the wet skid resistance, wet traction and snow behavior remain at the same level.

As advances in tyre design and tread compounding continue to provide lower rolling resistance tyres, reducing the hysteresis of the tyre sidewall also increases in importance. The sidewall contributes from $5 \%$ to $20 \%$ of the rolling resistance of the tyre, and can be changed relatively independently of the tyre design.

Waddell and coworkers ${ }^{56}$ studied the influence of adding precipitated silica to the black sidewall compound. They found that the use of silica lowers the hysteresis of a model black sidewall formulation containing natural and polybutadiene rubbers. Moreover, the use of precipitated silica significantly increased the compound tear strength, cut growth resistance and ozone ageing resistance. This happened without affecting scorch and cure time. According to these researchers, silica can be used as a part-per-part replacement of carbon black to the level of circa $10 \mathrm{phr}$.

As already discussed before, the mechanical properties of EPDM/diene rubber blends is severely deteriorated due to non-uniform distribution of crosslinks between the elastomer phases and inadequate interfacial crosslinks. The problem is further accentuated in non-black compounds, especially where silica is used as reinforcing filler. Silica containing a large amount of silanol $(\mathrm{Si}-\mathrm{OH})$ functional groups, retards cure by adsorbing polar curative molecules and by their acidic properties neutralising the preferentially alkaline vulcanisation chemistry. It has been observed that bis(diisopropyl) thiophosphoryl disulphide (DIPDIS) can be effectively used as a coupling agent and accelerator to co-vulcanise EPDM/NR blends ${ }^{57}$. Silanol groups of silica are capable of reacting with DIPDIS. The filler-curative adducts thus formed are probably more reactive for vulcanisation and result in faster curing with efficient rubber-silica coupling ${ }^{41}$.

\subsection{EPDM in Tyre Sidewalls}

The use of ozone resistant polymers for sidewall applications has received substantial attention in the last two decades to address the growing demands for glossy black tyre sidewalls. Polymers useful for this application are ethylenepropylene-diene terpolymers (EPDM), halogenated butyl rubbers (CIIR/BIIR) and brominated isobutylene-co-para-methyl styrene (BIMS). These rubbers are used in limited quantities, along with general-purpose highly unsaturated rubbers such as $\mathrm{NR}, \mathrm{SBR}, \mathrm{BR}$ in sidewall formulations, such that the dispersed domains of these rubbers effectively block the ozone-initiated cracks in the unsaturated rubber matrix. NR/BR/EPDM blends have been studied extensively in black tyre sidewall formulations. 


\subsubsection{Natural Rubber (NR)}

Natural rubber is obtained from the milky substance: latex, tapped from the "Rubber Tree", mainly grown in Southeast Asia. Chemically, NR is cis-1, 4polyisoprene with the structure in Figure 2.9.

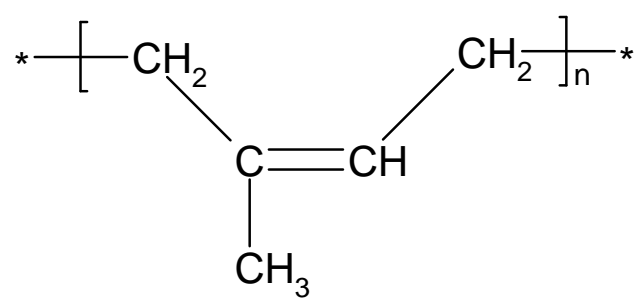

Figure 2.9 Structure of NR (Poly-cis-isoprene).

Because of the low enthalpy of melting or crystallisation $\Delta \mathrm{H}_{m}$ for $N R$, straining a piece of NR leads to a rise of the crystallisation temperature to above room temperature. This leads to a very important property: strain-crystallisation. This strain-crystallisation gives extra strength upon loading, which still occurs in the vulcanised state. In combination with the large number of unsaturated double bonds which gives a high vulcanisation yield, NR-vulcanisates show exceptionally high tensile and tear strength. The high abrasion resistance, a very useful property for tyres, can also be explained by these characteristics of NR.

Although there are now various kinds of synthetic rubbers available, NR is still used extensively in tyres, particularly in heavy-duty truck tyres. The main properties of NR are shown in the following table.

Table 2.3 Characteristics of NR.

\begin{tabular}{cc}
\hline Advantages & Disadvantages \\
\hline Tear Strength & Poor Uniformity of Quality \\
Wear Resistance & Poor Aging Resistance \\
Impact Resilience & Poor Fatigue Resistance \\
Low Heat Build-up & Poor Ozone Resistance \\
\hline
\end{tabular}

\subsubsection{Butadiene Rubber (BR)}

Butadiene rubber is also a common synthetic rubber used in tyres. BR has various butadiene-isomer structures in the molecular chain, which have a different influence on the final properties. There are three possibilities to build the butadiene monomers into the chain: Figure 2.10:

$$
\begin{aligned}
& \text { - 1,4 cis addition } \\
& \text { - 1,4 trans addition } \\
& \text { - 1,2 addition = vinyl-type }
\end{aligned}
$$




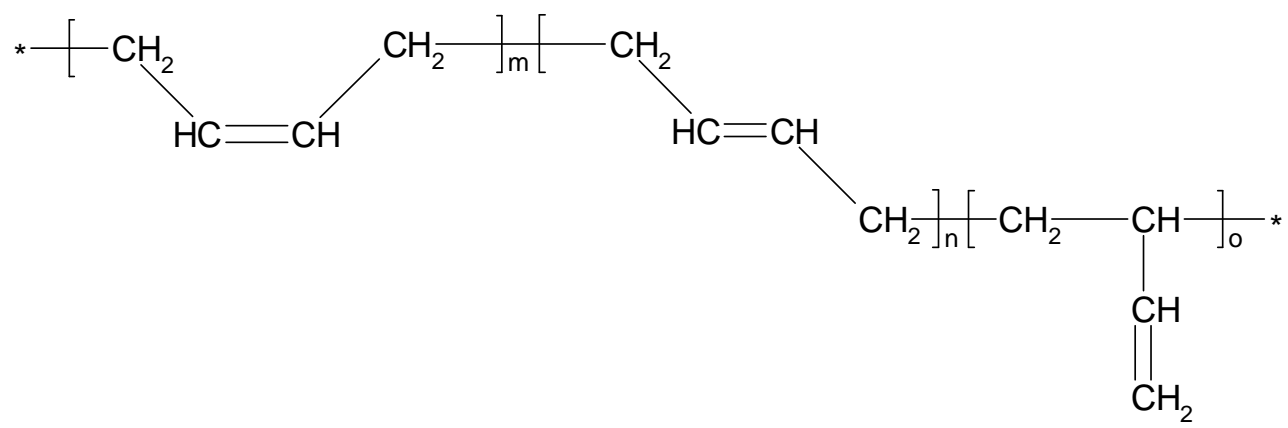

Figure 2.10 Structure of BR (Three different types of linkages: cis-1,4; trans-1,4; 1,2-vinyl).

The ratio between the cis- and trans-configurations depends on the catalyst system chosen. Normally, the preference is for a high cis-content for the best elastic properties. Shershnev ${ }^{58}$ studied the influence of 1,2-units and 1,4-units in butadiene on the formation of crosslinks and in blends with cis-1,4-polyisoprene. He stated that, as the content of 1,2-units increases, the miscibility of BR in NR increases.

Zhao and Ghebremeskel ${ }^{59}$ did an investigation into the fracture and fatigue properties of BR. They concluded that with increasing vinyl-content of the BR, the rubber shows high viscoelastic dissipation and therefore delays fatigue failure by means of this viscoelastic energy dissipation. On the other hand, tensile strength and strain at break are strongly dependent on the cis-content of the polymer. For instance at $5^{\circ} \mathrm{C}$, the BR type with a cis-content of $98,5 \%$ shows a higher increase in tensile strength and ultimate elongation upon increasing network density than BR with a low cis-content of $43 \%$, because the high cis-structure introduces some strain-induced crystallisation, which acts as reinforcing domains.

$\mathrm{BR}$ is used in large amounts in tread recipes for trucks and passenger cars, although never in its pure form. Unlike NR, there is little interaction among the BR molecules. For this reason, a compound of BR has high flexibility but poor elongation resistance. BR has good resistance to both wear and low-temperatures. Therefore, it is generally used for mixing with NR to compensate for its disadvantages.

Table 2.4 Characteristics of BR.

\begin{tabular}{cc}
\hline Advantages & Disadvantages \\
\hline Impact Resilience & \\
Wear Resistance & Poor Tear Strength \\
Low Temperature Properties & \\
Fatigue Resistance & \\
\hline
\end{tabular}




\subsubsection{Ethylene-Propylene-Diene Terpolymer (EPDM)}

\subsubsection{Introduction to EPDM}

Ethylene-propylene polymers are widely used synthetic rubbers. They are particularly known for their excellent resistance to ozone and ageing in comparison with natural rubber and common high-diene synthetic rubbers.

Unsaturated ethylene-propylene-diene elastomer consists of ethylene and propylene units as part of the main polymer chain and contains a diene as the third monomer. The third monomer introduces a carbon-carbon double bond pendant to the backbone chain of the polymer, which itself is completely saturated. Normally three common third monomers are employed in EPDM: ethylidene norbornene (ENB), dicyclopentadiene (DCPD) and hexadiene (HD): Figure 2.11. The use of HD has been stopped recently with the closure of a dedicated plant. The structure of a ENB-type EPDM is shown in figure 2.12.

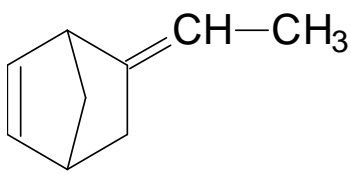

(a)

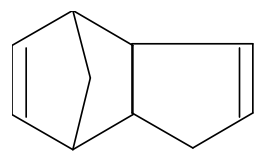

(b)

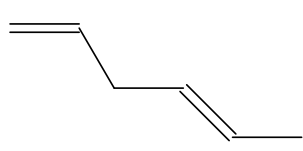

(c)

Figure 2.11 Structures of diene monomers in EPDM: (a) ENB; (b) DCPD; (c) HD.

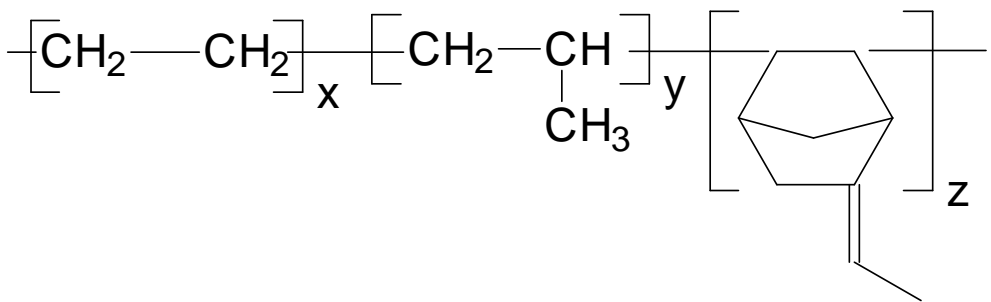

Figure 2.12 Structure of EPDM (ENB containing): $x \sim 1500 ; y \sim 750 ; z \sim 20$.

The saturated backbone of EPDM is the main structural feature that provides this rubber with excellent weather and ozone resistance. Sites of unsaturation in the polymer are the primary points of attack for oxidants. Commercial EPDM contains between 0 to $10 \mathrm{wt} \%$ non-conjugated diene and therefore contains significantly less unsaturation than the general high-diene rubbers ${ }^{60-62}$.

On the other hand, the hydrocarbon nature of EPDM leads to relatively poor chemical reactivity, low polarity and surface energy of the polymer and hence it is difficult to bond ${ }^{63}$. Also, EPDM interacts less strongly with carbon black than highdiene rubbers.

The characteristics of EPDM greatly depend on its structure. The kind and amount of diene, the ethylene-propylene ratio, the molecular weight and molecular 
weight distribution of EPDM polymers all play important roles. So the intended enduse applications are generally the determining factor in the selection of a particular grade of EPDM.

\subsubsection{Protection mechanism of EPDM against ozone}

The mechanism of protection against ozone-induced cracking of highly unsaturated rubbers by the incorporation of essentially saturated polymers such as EPDM, has been proposed by Andrews ${ }^{64}$. It is believed that EPDM is present in particles or regions dispersed in the high-diene rubber phase. Ozone attacks the sidewall, generating micro-cracks in the high-diene rubber phase, to continue until the crack tip encounters an EPDM region. Then the crack propagation is interrupted by the small EPDM particles. However, if a propagating microcrack is too large when it reaches a particle of EPDM, there will be sufficient stress concentration for the growing crack to "jump around" the ozone-resistant crack-path obstructions, the EPDM particles. Thus, there should be enough EPDM particles to prevent the microcracks from growing to a sufficient length to give the stress concentration required for "obstruction jumping" 34 .

Andrew's theory is based on what would now be called "crack bridging": the growing crack meets a fracture resistant, ozone inert particle and must either circumvent it within the plane of the propagating crack, leaving the crack faces pinned together at the particle, or propagate around it by interfacial cracking and deviating out of the fracture plane. It is said that the stress intensity in front of the crack tip is reduced by the particles pinning the faces of the crack together behind its tip.

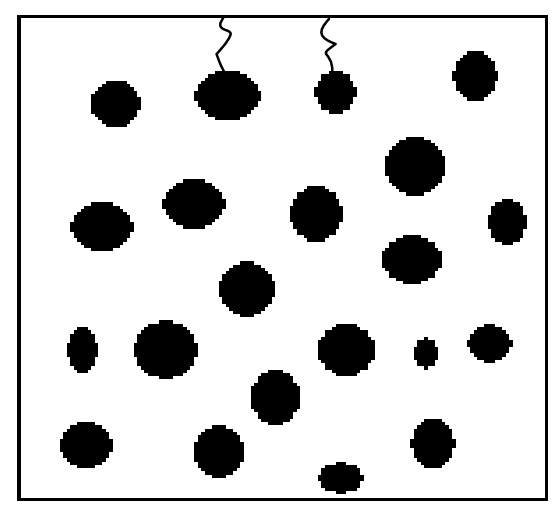

Figure 2.13 Morphology of EPDM particles in a high diene rubber phase; smaller particles bring better ozone crack resistance.

The influence of the size and the distribution of the dispersed EPDM particles was studied by Ban and coworkers ${ }^{65}$. They found that the better ozone crack 
resistant compounds had considerably smaller particles. Ban stated that this observation seemed in accordance with the theory of Andrews. In this way, there is a higher concentration of 'crack stoppers' per unit of length, when the EPDM particles are smaller and better dispersed. As the stress at the tip of the crack is related to the crack length in the reactive phase, a higher density of particles will reduce the potential for a crack to jump over the inert particles and grow to a macroscopic crack. Hence, the critical stress for the onset of ozone stress cracking will be higher.

Another mechanism of ozone protection of EPDM has been proposed by Doyle $^{66}$. He states that the observations in his experiments are not consistent with the mechanism of Andrews. Doyle suggests that the major effect of the EPDM is on the apparent crack initiation condition. The presence of a dispersion of ozone resistant particles impedes the rate at which damaged (ozonized) regions of the NR network can nucleate voids which then coalesce to initiate macroscopic cracks. Since damage only occurs in the NR phase, statistical fluctuations in the morphology play an important role in crack nucleation. Consistent with his observations the resistance to ozone is predicted to improve with homogeneity of the phase dispersion and as the size of the dispersed phase domains decreases. Sub-inclusions of the matrix phase were observed in many of the blend compounds. The effective phase volume of the inert phase is not rigidly defined by the fractional composition of the inert component; mixing procedures and curative kinetics could have an effect on this aspect of the final blend morphology and thus the ozone resistance.

\subsubsection{Influence of EPDM on properties of NR/BR/EPDM blends}

It is a common experience that about 30-40 phr EPDM is required in conventional tyre sidewall compounds in order to "repair" for the loss of ozone resistance by the omission of the antiozonants ${ }^{2}$. Sumner ${ }^{67}$ stated that the ozone resistance of a NR/EPDM blend depends on the level of EPDM. The higher the volume ratio of EPDM, the better is the resistance against ozone attack. Researchers in the past have found that with $40 \%$ EPDM there is no ozone cracking throughout the life of a sidewall. This has been shown in both dynamic and static tests ${ }^{39}$. On the other hand, it is desirable to maximize the amount of high-diene rubber in terms of costs and mechanical performance.

The various grades of EPDM with variants of diene type, diene level, ethylene content and molecular weight provide significantly different vulcanised properties of EPDM/diene rubber blends as well. Traditionally, good properties of EPDM/diene blends have been sought in selecting EPDM grades of high molecular weight with a high unsaturation level. These grades not only reduce the cure mismatch but also substantially improve the adhesion to a carcass compound, the aged fatigue life and the dynamic ozone resistance. 
Since EPDM rubbers are much lower in unsaturation than high-diene rubbers, a rather basic method of improving blends of these two rubbers would be to increase the unsaturation of EPDM so that its rate of cure is closer to that of diene rubbers. Therefore, blends of these two rubbers would have better cure rate compatability. Thus, it is expected that high unsaturation EPDM diene rubber blends would have better properties than low unsaturation EPDM diene rubber blends.

Moreover, an increase of the Mooney viscosity and/or the ENB-content of the EPDM results in an increase of the crosslink density of the EPDM phase in covulcanisation. In both cases, the number of potential crosslink sites per EPDM chain is increased and the chance of linking EPDM chains to the rubber network is enhanced $^{68}$. In Hellens' ${ }^{69}$ work, he found that in NR/EPDM blends the cure state of an ENB-type of EPDM increases with the ENB-level and molecular weight.

However, even the so-called "very highly" unsaturated EPDM grades still have less than $10 \%$ of the unsaturation of $\mathrm{NR}$ on a molar basis. Furthermore, high molecular weight is reflected in a very high Mooney viscosity, which leads to mixing problems. A poor dispersion of EPDM in the other rubber components will negatively influence the beneficial effects of the EPDM. Therefore, van de Ven and Noordermeer ${ }^{53}$ have stated that the dispersion of the various rubber phases along with the carbon black dispersion is much more important than the cure incompatibility.

As stated, the dispersion of EPDM in the high-diene matrix is essential for the protection of the elastomer blend. To solve this problem, Hong and Ferrandino ${ }^{70}$ used low molecular weight EPDM (liquid EPDM) in tyre compounds. The low viscosity of liquid EPDM enhances the diffusion of the high-viscosity EPDM into the high-diene phase and reduced the processing cost as well. They found that the replacement of low molecular weight EPDM in a NR/BR/EPDM black sidewall provided adequate dynamic ozone cracking resistance, but may also improve polymer dispersion and cut growth resistance. The significant improvements in cut growth resistance would promote this compound as a good candidate for a better appearance black sidewall.

\section{REFERENCES}

1. Adhikary, A.; Mukhopadhyay, R., Rubber World, 230, 16 (2004).

2. Waddell, W. H., Rubber Chem. Technol., 71, 590 (1998).

3. Cox, W. L., Rubber Chem. Technol., 32, 364 (1959).

4. Layer, R. W., Rubber Chem. Technol., 39, 1584 (1966).

5. Hofmann, W., "Rubber Technology Handbook", Hanser Publishers, Munich, 1989.

6. Loan, L. D., J. Int. Rubber Technol., 2, 73 (1968).

7. Braden, M.; Gent, A. N., Rubber Chem. Technol., 35, 200 (1962).

8. Huntink, N. M., Thesis, University of Twente, Enschede, 2003. 
9. Avgeropoulos, G. N.; Weissert, F. C., Rubber Chem. Technol., 49, 93 (1976).

10. Bhowmick, A. K.; De, S. K., Rubber Chem. Technol., 53, 960 (1980).

11. Chang, Y.; Shin, Y., J. Appl. Polym. Sci., 73, 749 (1999).

12. Hildebrand, J. H.; Scott, R. L., "The Solubility of Nonelecrolytes", Dover Publications, New York, 1964.

13. Klüppel, M.; Schuster, R. H.; Schaper, J., Rubber Chem. Technol., 72, 91 (1999).

14. Deutsches Institut Für Kautschuktechnologie, "Einfluss von Prozessparametern auf die Phasenmorphologie von Kautschukverschnitten", Deutsche Kautschuk-Gesellschaft e.V., Hannover, 1998.

15. Paul, D. R.; Newman, S., "Polymer Blends", Academic Press, New York, 1978.

16. Young, D. G.; Kresge, E. N.; Wallace, A. J., Rubber Chem. Technol., 55, 428 (1982).

17. Setua, D. K.; White, J. L., Polym. Eng. Sci., 31, 742 (1991).

18. Setua, D. K.; Pandey, K. N., J. Appl. Polym. Sci., 74, 480 (1999).

19. Zanzig, D. J.; Magnus, F. L., Rubber Chem. Technol., 66, 538 (1993).

20. Oliveira, M. G.; Gomes, A. C. O., Macromol. Chem. Phys., 205, 465 (2004).

21. Ismail, H.; Supri; Yusof, A. M. M., J. Appl. Polym. Sci., 96, 2181 (2005).

22. Zhao, J.; Ghebremeskel, G., Kautsch. Gummi Kunstst., 3, 84 (2001).

23. Chapman, A. V.; Tinker, A. J., Kautsch. Gummi Kunstst., 56, 533 (2003).

24. Ignatz-Hoover, F.; To, B. H.; Datta, R. N.; Hoog, A. J. d.; Huntink, N. M.; Talma, A. G., Rubber Chem. Technol., 76, 747 (2003).

25. Guo, R.; Talma, A. G.; Datta, R. N.; Dierkes, W. K.; Noordermeer, J. W. M., Eur. Pol. J., 44, 3890 (2008).

26. Hess, W. M.; Herd, C. R.; Vegvari, P. C., Meeting of the Rubber Division, American Chemical Society, Nashville, Tennessee, November, 1992.

27. Gardiner, J. B., Rubber Chem. Technol., 43, 370 (1970).

28. Gardiner, J. B., Rubber Chem. Technol., 41, 1312 (1968).

29. Gardiner, J. B., Rubber Chem. Technol., 42, 1058 (1969).

30. Gullaumond, F. X., Revue Generale des Caoutchoucs et Plastiques, 51, 853 (1974).

31. Brown, P. S.; Tinker, A. J., J. Nat. Rubber Res., 5, 157 (1990).

32. Woods, M. E.; Davidson, J. A., Rubber Chem. Technol., 49, 112 (1976).

33. Shershnev, V. A., Rubber Chem. Technol., 55, 537 (1982).

34. Coran, A. Y., Rubber Chem. Technol., 61, 281 (1988).

35. Oliveira, M. G.; Soares, B. G., Macromol. Rapid Commun., 20, 526 (1999).

36. Hopper, R. J., Rubber Chem. Technol., 49, 341 (1976).

37. Baranwal, K. C.; Son, P. N., Rubber Chem. Technol., 47, 88 (1974).

38. Morrissey, R. T. Meeting of the Rubber Division, American Chemical Society, Miami, Florida, April, 1971.

39. Cook, S., "Blends of Natural Rubber", Chapman \& Hall, 1998.

40. Suma, N.; Joseph, R.; George, K. E., J. Appl. Polym. Sci., 49, 549 (1993).

41. Ghosh, A. K.; Basu, D. K., Kautsch. Gummi Kunstst., 56, 101 (2003). 
42. Sahakaro, K.; Naskar, N.; Datta, R. N.; Noordermeer, J. W. M., J. Appl. Polym. Sci., 103, 2538 (2007).

43. Sahakaro, K.; Talma, A. G.; Datta, R. N.; Noordermeer, J. W. M., J. Appl. Polym. Sci., 103, 2547 (2007).

44. Sahakaro, K.; Datta, R. N.; Baaji, J.; Noordermeer, J. W. M., J. Appl. Polym. Sci., 103, 2555 (2007).

45. Brodsky, G. I. Meeting of the Rubber Division, American Chemical Society, Orlando, Florida, October, 1993.

46. Zhao, J.; Ghebremeskel, G., Kautsch. Gummi Kunstst., 54, 223 (2001).

47. Dannenberg, E. M., Rubber Chem. Technol., 48, 410 (1975).

48. Choi, S. S., Polym. Adv. Technol., 13, 466 (2002).

49. Noordermeer, J. W. M., :Industrial Elastomers", University of Twente, 2005.

50. O'Farrell, C. P.; Gerspacher, M.; Yang, H., Meeting of the Rubber Division, American Chemical Society, Nashville, Tennessee, 1992.

51. Callan, J. E.; Hess, W. M.; Scott, C. E., Rubber Chem. Technol., 44, 814 (1971).

52. Cotten, G. R., Rubber Chem. Technol., 48, 548 (1975).

53. van de Ven, P.M.; Noordermeer, J. W. M., Rubber World, 222, 55 (2000).

54. Herd, C. R.; Bomo, F.; Swartz, L., Kautsch. Gummi Kunstst., 48, 588 (1995).

55. Cotten, G. R.; Murphy, L. J., Rubber Chem. Technol., 61, 609 (1988).

56. Waddell, W. H.; Douglas, J. B.; Okel, T. A.; Snodgrass, L. J., Rubber World, 21-25 (1993).

57. Ghosh, A. K.; Basu, D. K., Asia Rub. Tech. Expo., 2002.

58. Shershnev, V. A.; Shundrina, I. K., J. Polym. Sci., 39, 10 (1997).

59. Zhao, J.; Ghebremeskel, G., Rubber and Plastic News, 14 (November 27, 2000).

60. Karpeles, R.; Grossi, A. V., :Handbook of Elastomers", Marcel Dekker Inc., New York, Basel, 2001.

61. Noordermeer, J. W. M. in "Kirk-Othmer Encyclopedia of Chemical Technology", 5th ed.; Wiley \& Sons, New York, 2002.

62. Noordermeer, J. W. M. in "Encyclopedia of Polymer Science and Technology", 3rd ed.; Wiley \& Sons, New York, 2002.

63. Markovic, M. G.; Choudbury, N. R., Kautsch. Gummi Kunstst., 52, 170 (1999).

64. Andrews, E. H., Rubber Chem. Technol., 40, 635 (1967).

65. Ban, L. L.; Doyle, M. J.; Smith, G. R. Meeting of the Rubber Division, American Chemical Society, Cleveland, Ohio, 1985.

66. Doyle, M. J. I.T.E.C., Akron, OH., 10-12 Sept. 1996.

67. Sumner, A. J. M.; Fries, H., Kautsch. Gummi Kunstst., 45, 558 (1992).

68. van Duin, M.; Krans, J. C. J.; Smedinga, J., Kautsch. Gummi Kunstst., 46, 445 (1993).

69. Hellens, W. v., Kautsch. Gummi Kunstst., 47, 124 (1994).

70. Hong, S. W.; Ferrandino, M. P., Kautsch. Gummi Kunstst., 51, 88 (1998). 


\title{
Chapter 3
}

\section{Studies on EPDM Modification by N-Chlorothio-N- Butyl-Benzenesulphonamide}

\begin{abstract}
$\mathrm{N}$-chlorothiosulphonamides have been used to modify EPDM in order to enhance the compatibility of EPDM in e.g. NR/BR/EPDM blends for ozone resistance. N-Chlorothio-N-ButylBenzenesulphonamide (CTBBS) was selected as a representative for N-chlorothiosulphonamides. In this study, it was found that CTBBS behaves differently with various types of EPDM. Three types of EPDM were selected: ENB-EPDM, HD-EPDM and DCPD-EPDM. HD-EPDM showed the highest effectiveness towards CTBBS-grafting. However, this EPDM is not commercially available anymore. On the opposite side, DCPD-EPDM showed the lowest reactivity so that almost no grafting could be realized. The result with ENB-EPDM was that upon application of CTBBS to ENB-EPDM, gelation occurred and therefore, a low amount of grafting was achieved. With the limited grafting efficiency for ENBEPDM, there is no significant improvement when applying the grafted ENB-EPDM into NR/BR/EPDM blends.
\end{abstract}




\subsection{Introduction}

Ethylene propylene diene rubber (EPDM) is a highly saturated elastomer, which is widely used in applications that require good ozone resistance. It has long been known that the ozone resistance of high-diene rubbers such as natural rubber (NR), styrene-butadiene rubber (SBR), nitrile rubber (NBR) and butadiene rubber (BR) can be greatly improved by the incorporation therein of EPDM ${ }^{1}$. However, the vulcanisates of such elastomer blends are generally poor in both static and dynamic mechnical properties, such as ultimate strength-related properties, fatigue resistance, hysteresis, etc.

The main problems associated with the generally poor properties of EPDM/high-diene rubber blends are the large differences in the chemical reactivity of EPDM and high-diene rubbers. The difference in molar concentrations of double bonds in each of the elastomers results in a difference in polarity and the number of allylic sites for sulphur vulcanisation to obtain crosslinks ${ }^{2}$. Such a difference is reflected in the relative rates of crosslinking during vulcanisation, diffusion of curatives towards the faster curing high-diene phase and a heterogeneous filler distribution between the rubber phases ${ }^{3-7}$.

There have been several attempts to improve the properties of EPDM/polydiene blends. Generally, these attempts have sought to increase the cure rate of EPDM, either by using curatives that have an increased reactivity towards EPDM, or by means of modifying the EPDM to make it more reactive towards curing ${ }^{8-}$ 14. Coran ${ }^{10}$ achieved better cure compatibility for EPDM/NR blends by modifying the EPDM with maleic anhydride. Oliveira and Soares ${ }^{11}$ studied the functionalization of EPDM with mercapto-groups. Baranwal and Son ${ }^{12}$ modified EPDM in solution with accelerator species, like the sulphur donor dithiodimorpholine (DTDM), using UVirradiation in the presence of a photosensitizer. Morrissey ${ }^{13}$ reported, that halogenation of EPDM in solution could result in a marked improvement in its cure compatibility with highly unsaturated rubbers. Hashimoto et al. ${ }^{14}$ prepared a modified EPDM in solution to achieve bound 2-mercaptobenzothiazole EPDM.

Hopper ${ }^{15-18}$ modified EPDM with $\mathrm{N}$-chlorothiosulphonamides in solution and melt to give pendent groups which would act like a retarder to high-diene vulcanisation. Blends of the modified EPDM with synthetic polyisoprene (IR) exhibited higher modulus and tensile strength together with lower heat build-up, and this was taken as evidence of improved vulcanisation of EPDM. As there are different types of EPDM in existence as ethylidene norbornene (ENB)-EPDM, hexadiene (HD)-EPDM and dicyclopentadiene (DCPD)-EPDM, a study on the selectivity of $\mathrm{N}$ chlorothiosulphonamides towards these various EPDMs is of great interest. The objective of this work was therefore to study the reactivity of $\mathrm{N}$ - 
chlorothiosulphonamides towards these various EPDMs and their influence on vulcanisation behaviour in blends with highly unsaturated rubbers.

\subsection{Experimental}

\subsubsection{Materials}

N-chlorothio-N'-butyl-benzenesulphonamide (CTBBS) was prepared using nbutylamine (99.5\%, Aldrich), triethylamine (99.5\%, Aldrich), dichloromethane $(99.9 \%$, Biosolve), benzenesulphonyl chloride (99.0\%, Aldrich), sulphurdichloride (98\%, Fluka) and diethyl ether (99.8\%, Fluka).

The elastomers selected for the blend were natural rubber (SIR20, Standard Indonesian Rubber), butadiene rubber (Kosyn KBR01, Korea Kumho Petrochemical Co., Ltd) and four types of EPDM rubber: Table 3.1. The other compounding ingredients used were extra pure grade zinc oxide (Merck, Germany), finely divided sulphur (Merck, Germany), 95\% pure stearic acid (Aldrich, Germany), poly(2,2,4trimethyl-1,2-dihydroquinoline) or TMQ (Flexsys B.V., the Netherlands) and Ncyclohexyl-2-benzothiazolesulphenamide (CBS) or Santocure ${ }^{\circledR}$ (Flexsys B.V., the Netherlands).

Table 3.1 Types of EPDM rubbers empolyed.

\begin{tabular}{|c|c|c|c|}
\hline Material & $\begin{array}{l}\text { Structure of the third } \\
\text { monomer }\end{array}$ & Composition & Supplier \\
\hline $\begin{array}{l}\text { Keltan } 5508 \\
\text { (LENB-EPDM) }\end{array}$ & & $\begin{array}{l}4.5 w t \% \text { ethylidene- } \\
\text { norbonene (ENB) } \\
70 w t \% \text { ethylene }\end{array}$ & \\
\hline $\begin{array}{l}\text { Keltan } 4703 \\
\text { (HENB- } \\
\text { EPDM) }\end{array}$ & $-\mathrm{CH}_{3}$ & $\begin{array}{l}9 w t \% \text { ethylidene-norbonene } \\
\text { (ENB) } \\
48 w t \% \text { ethylene }\end{array}$ & $\begin{array}{l}\text { DSM Elastomers } \\
\text { B.V., the } \\
\text { Netherlands }\end{array}$ \\
\hline $\begin{array}{l}\text { Keltan } 520 \\
\text { (DCPD- } \\
\text { EPDM) }\end{array}$ & & $\begin{array}{l}\text { 4.5wt\% Dicyclopentadiene } \\
\text { (DCPD) } \\
58 w t \% \text { ethylene }\end{array}$ & \\
\hline $\begin{array}{l}\text { Nordel } 1070 \\
\text { (HD-EPDM) }\end{array}$ & & $\begin{array}{l}\text { 4wt\% Hexadiene (HD) } \\
54 w t \% \text { ethylene }\end{array}$ & $\begin{array}{l}\text { Dupont, United } \\
\text { States }\end{array}$ \\
\hline
\end{tabular}




\subsubsection{Synthesis of N-chlorothio-N'-butyl-benzenesulphonamide (CTBBS)}

N-chlorothio-N'-butyl-benzenesulphonamide (CTBBS) was prepared by two steps as shown in Scheme 1. In the first step, $7.3 \mathrm{~g}$ n-butylamine and $10.1 \mathrm{~g}$ triethylamine were dissolved in $200 \mathrm{ml}$ dichloromethane. The solution was then cooled to $0^{\circ} \mathrm{C}$ in an ice bath. Afterwards, a solution of $17.6 \mathrm{~g}$ benzenesulphonyl chloride in $50 \mathrm{ml}$ dichloromethane was slowly added. The reaction mixture was stirred at room temperature for 2 hours and refluxed for 1 hour to complete the reaction. The reaction mixture was then washed several times with a diluted hydrochloric acid solution and pure water in a separation funnel to remove unreacted triethylamine and triethylamine hydrochloride. The organic layer was dried by Magnesium sulphate, filtered and evaporated. The remaining material was $\mathrm{N}$-butyl-benzenesulphonamide, which was characterized by NMR: 7.88-7.50ppm, 7.59-7.49ppm, 4.60ppm, 2.992.95ppm (quartet), 1.50-1.20ppm, 0.88-0.82ppm.

In the second step, $10.7 \mathrm{~g} \mathrm{~N}$-butyl-benzenesulphonamide and $5.1 \mathrm{~g}$ triethylamine were dissolved in $200 \mathrm{ml}$ diethyl ether and cooled to $0{ }^{\circ} \mathrm{C}$. Then a solution of $5.2 \mathrm{~g}$ sulphurdichloride in $50 \mathrm{ml}$ ether was added slowly while maintaining the temperature at $0^{\circ} \mathrm{C}$. During the reaction, a precipitate of triethylamine hydrochloride was formed and filtered afterwards. After evaporation of the ether, the residue was characterized by NMR as N-chlorothio-N'-butyl-benzenesulphonamide: 8.00-7.96ppm, 7.717.65ppm, 2.99-2.95ppm (triplet), 1.50-1.20ppm, 0.97-0.92ppm.

Step 1:

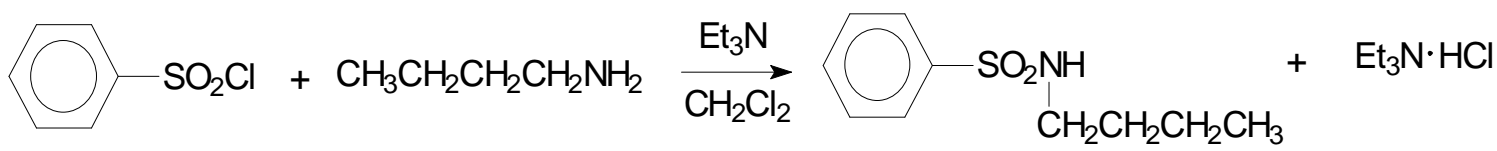

Step 2:

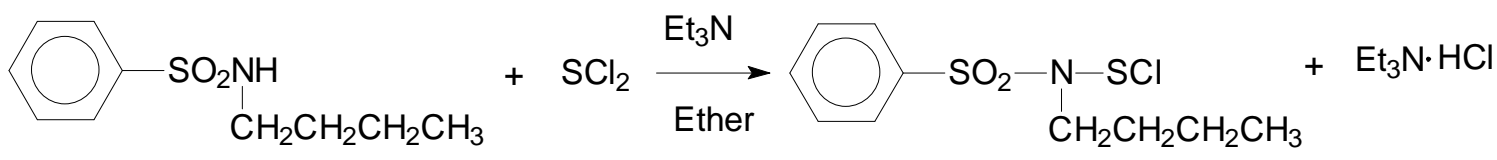

Scheme 3.1 Synthesis of N-chlorothio-N'-butyl-benzenesulphonamide (CTBBS).

\subsubsection{Grafting of CTBBS onto EPDM}

3.2.3.1 In solution 
5 grams of EPDM were first dissolved in $200 \mathrm{ml}$ hexane and flushed with nitrogen. For the ENB-type EPDM, a suspension of calcium stearate in dichloromethane was added to the polymer solution before reaction to act as acid acceptor for the reaction ${ }^{18}$. The amount of calcium stearate was equal on a molar basis to the amount of CTBBS to be added next. For HD-EPDM and DCPD-EPDM, this was omitted. Then a CTBBS-solution in dichloromethane was added slowly into the EPDM solution. The CTBBS-amounts added were varied, to correspond to relative ratios (\%) with respect to the total amount of third monomer available for reaction: Table 3.2.

Table 3.2 CTBBS-amounts added for the different types of EPDM.

\begin{tabular}{|c|c|c|c|c|c|c|c|}
\hline \multirow{2}{*}{ EPDMs } & \multirow{2}{*}{$\begin{array}{l}\text { Third monomer } \\
\text { (mmol/100g } \\
\text { EPDM) }\end{array}$} & \multicolumn{6}{|c|}{$\begin{array}{l}\text { CTBBS amounts relative to available unsaturation } \\
\qquad(\mathrm{mmol})\end{array}$} \\
\hline & & $10 \%$ & $20 \%$ & $30 \%$ & $40 \%$ & $50 \%$ & $60 \%$ \\
\hline LENB-EPDM & 11.3 & 1.13 & 2.26 & - & - & - & - \\
\hline HENB-EPDM & 22.5 & 2.25 & - & - & - & - & - \\
\hline HD-EPDM & 14.6 & 1.46 & 2.92 & 4.38 & 5.84 & 7.30 & 8.76 \\
\hline DCPD-EPDM & 10.2 & 1.02 & 2.04 & 3.06 & 4.08 & 5.10 & 6.12 \\
\hline
\end{tabular}

After stirring for several hours at ambient temperature, the final rubber sample was isolated by coagulation and washing with acetone, followed by drying in a vacuum oven. The time needed for the reaction to be completed and the highest CTBBS amount which can be added before gelation was different for the various types of EPDM, as shown in Table 3.3.

Table 3.3 Reaction times and highest CTBBS amounts that can be added before gelation for different types of EPDM.

\begin{tabular}{lcc}
\hline \multicolumn{1}{c}{ EPDM types } & Reaction time & Highest CTBBS amount \\
\hline LENB-EPDM & 4 hours & $20 \%$ \\
HENB-EPDM & 4 hours & $10 \%$ \\
HD-EPDM & 48 hours & $60 \%$ \\
DCPD-EPDM & No reaction & No reaction \\
\hline
\end{tabular}




\subsubsection{In internal mixer}

Grafting of CTBBS onto ENB-EPDM was also tried in a Brabender PL2000 with a chamber volume of $50 \mathrm{~cm}^{3}$, using a rotor speed of $80 \mathrm{rpm}$, a fill factor of 0.8 and an initial temperature varied between $100^{\circ} \mathrm{C}$ and $140^{\circ} \mathrm{C}$. The EPDM was first masticated for 1 minute, then calcium stearate in molar equivalents to CTBBS was added. After another 3 minutes of mixing, CTBBS was added and the mixing was continued for another 6 minutes. The final compound temperature before dumping from the mixer was in the range of $140^{\circ} \mathrm{C} \pm 10^{\circ} \mathrm{C}$. A sample for analysis was purified by dissolving in chloroform, coagulated and washed with acetone, then dried in a vacuum oven.

\subsubsection{Characterization of the grafted EPDMs}

\subsubsection{ATR-IR}

The infrared spectra of the grafted EPDM-samples were recorded by using the attenuated total reflectance infrared technique (ATR/IR) with a spectrometer FTS-60 (BIO-RAD Laboratories B.V., the Netherlands). The infrared spectra were recorded with 512 scans at a resolution of $8 \mathrm{~cm}^{-1}$ in the range of $4000-600 \mathrm{~cm}^{-1}$.

\subsubsection{NMR}

The ${ }^{1} \mathrm{H}-\mathrm{NMR}$ spectra of the grafted EPDM-samples were recorded with a Varian Unity $300 \mathrm{MHz}$ system at $25^{\circ} \mathrm{C}$ with $\mathrm{CDCl}_{3}$ as the solvent.

\subsubsection{Preparation of blends, vulcanisation and tensile test}

NR/BR/EPDM blends were prepared in a Schwabenthan two-roll mill. The recipes are shown in Table 3.4. NR was first masticated for 1 minute, then BR and EPDM (or grafted EPDM) were added and mixed for another 2 minutes. Subsequently, ZnO and TMQ were added. After 2 minutes of mixing, CBS and sulphur were added and the mixing was continued for another 2 minutes. The final compound was sheeted out. 
Table 3.4 Formulation of NR/BR/EPDM compounds.

\begin{tabular}{lc}
\hline \multicolumn{1}{c}{ Ingredient } & Content (phr) \\
\hline NR & 35 \\
BR & 35 \\
EPDM (grafted EPDM) & $30-32.5$ \\
Stearic acid & 2 \\
Zinc Oxide & 4 \\
TMQ & 1 \\
CBS & 1.98 \\
Sulphur & 2.5 \\
\hline
\end{tabular}

The optimum cure time $\left(t_{c, 90}\right)$ of the fully compounded gum blends was determined as the time needed to reach $90 \%$ of the maximum torque in a RPA 2000 (Alpha Technologies) at $150^{\circ} \mathrm{C}, 0.833 \mathrm{~Hz}$ and 0.2 degree strain. The blends were then vulcanised into $2 \mathrm{~mm}$ thick plates for the optimum cure time $t_{c, 90}$ in a Wickert WLP 1600 laboratory compression press at $150^{\circ} \mathrm{C}$ and 100 bar pressure.

Tensile tests were carried out according to ISO 37, with a Zwick tensile tester Model Z 1.0/TH1S at a constant crosshead speed of $500 \mathrm{~mm} / \mathrm{min}$.

\subsection{Results and Discussion}

\subsubsection{Grafting of CTBBS onto different types of EPDM in solution}

Grafting of CTBBS onto EPDM was first tried by the solution technique. The reacted EPDM was then characterized by ATR and NMR to check whether grafting occured.

\subsubsection{ENB-EPDM}

Figure 3.1 shows the ATR infrared spectra of CTBBS-grafted ENB-EPDM in comparison with virgin EPDM. CTBBS-grafted EPDM clearly shows some additional peaks. As the samples were washed with acetone prior to the ATR-measurement, the presence of above absorption peaks indicates that CTBBS-fragments are indeed grafted onto ENB-EPDM. These peaks and their assigned functional groups are listed in Table 3.5. 


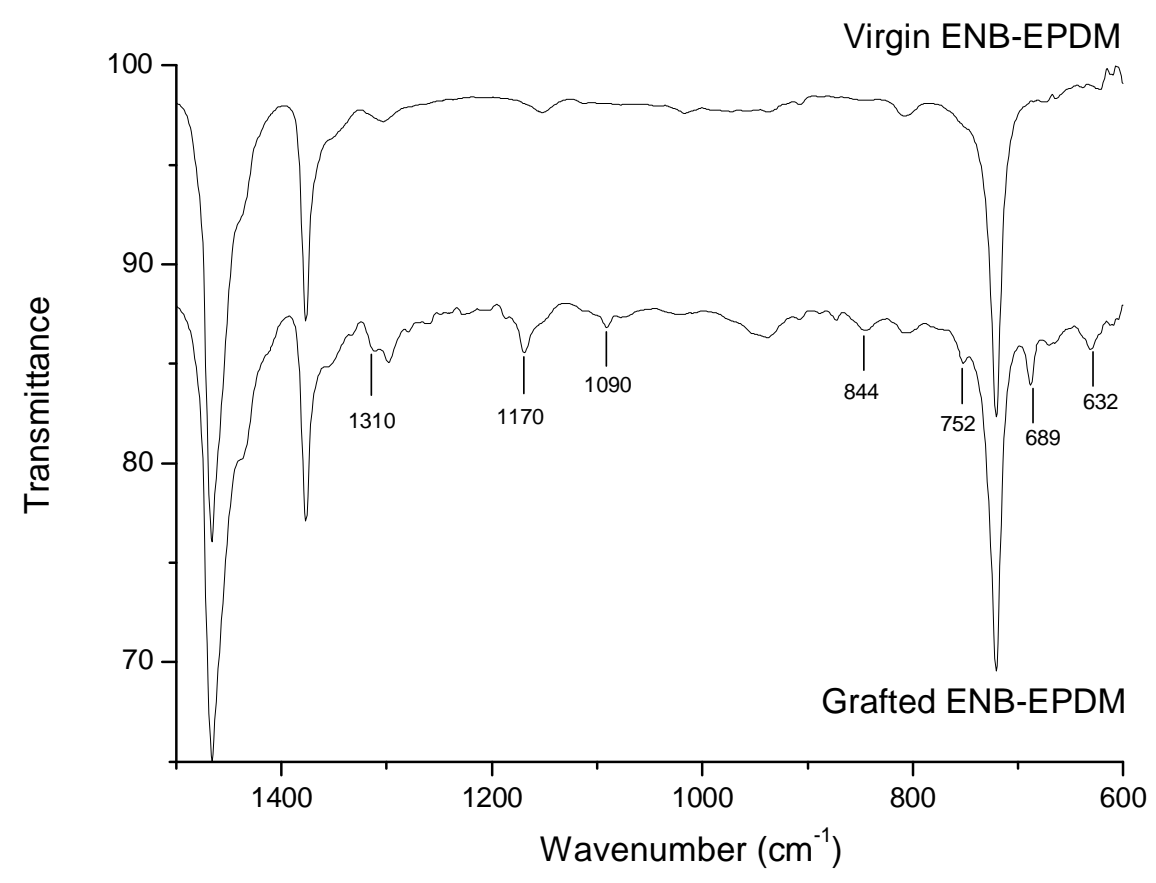

Figure 3.1 ATR/IR-spectra of CTBBS-grafted ENB-EPDM in comparison with virgin ENBEPDM.

Table 3.5 Infrared absorption peaks and their assignments.

\begin{tabular}{cc}
\hline Infrared absorption peak $\left(\mathrm{cm}^{-1}\right)$ & Functional group \\
\hline 1310,1090 & - \\
1170 & -
\end{tabular}

As the relevant peaks partly overlap with peaks of EPDM, they are not suited for a quantification of the grafting. Therefore, an attempt was made to obtain more information from the $\mathrm{H}^{1}$-NMR spectrum of the samples. The NMR spectra of CTBBSgrafted and virgin ENB-EPDM are shown in figure 3.2. Compared to virgin EPDM, the grafted EPDM shows additional peaks in the range from $7.4 \mathrm{ppm}$ to $8 \mathrm{ppm}$ which can be attributed to the aromatic protons from CTBBS. Two peaks at 5.0ppm and 
5.22ppm are due to the olefinic vinyl hydrogen of the ENB group with two different configurations $^{197}$. The peak at $0.8 \mathrm{ppm}$ is from large amount of methyl-groups in EPDM. As the amount of methyl groups will not have changed after the grafting reaction, this peak at $0.8 \mathrm{ppm}$ was defined as reference peak. Then, the integration area of the peaks of vinyl hydrogen from the ENB group can be quantified, remaining after the grafting with CTBBS.

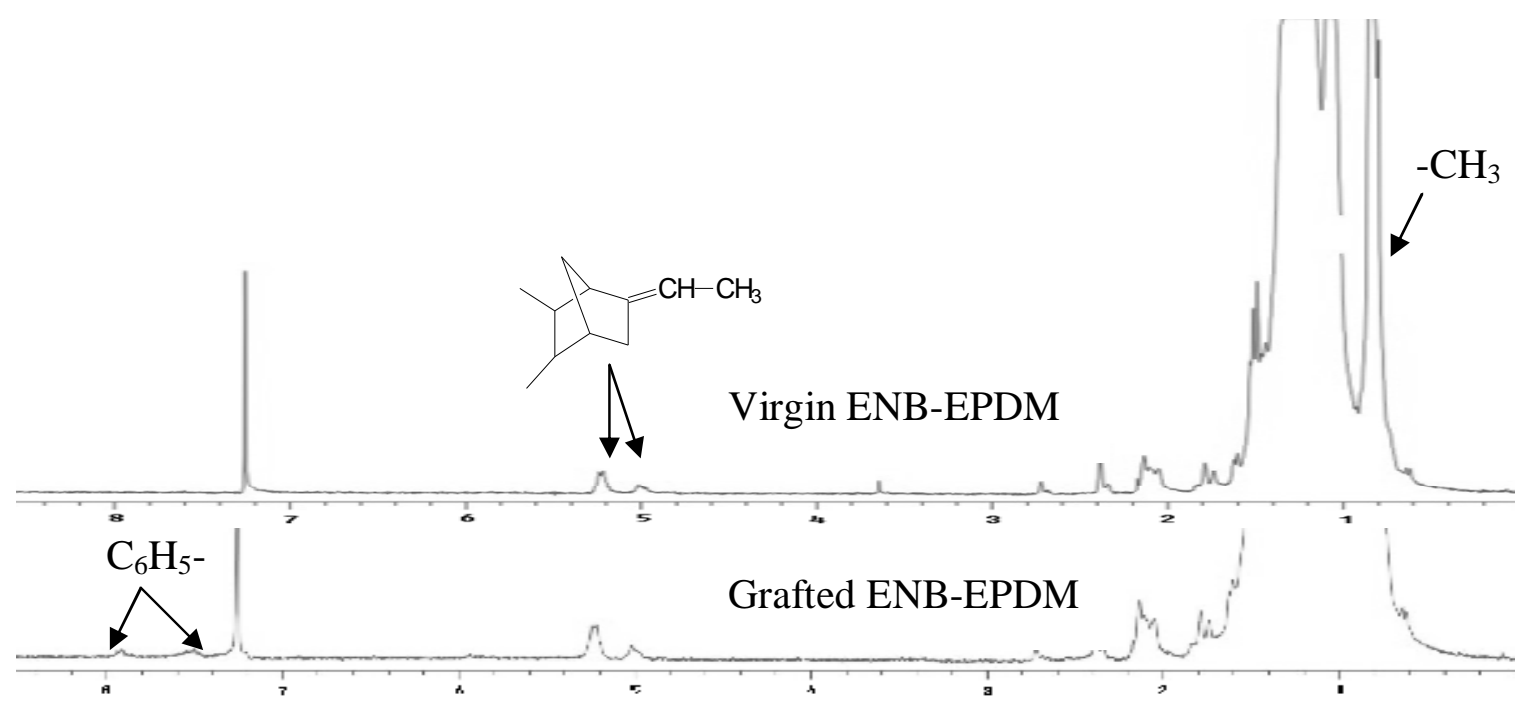

Figure 3.2 NMR-spectra of CTBBS grafted ENB-EPDM in comparison with virgin EPDM.

After CTBBS-grafting, the peak area of residual olefinic protons decreases. By changing the CTBBS/EPDM ratio, a calibration curve of olefinic proton peak area versus CTBBS-amount could be obtained as in figure 3.3. For LENB-EPDM, when the CTBBS-amount increased to $30 \%$, the viscosity of the polymer solution increased significantly and the solution became completely gelled 15 minutes after CTBBS addition. As HENB-EPDM contains a twice higher amount of ENB, it is even more sensitive to become gelled. For HENB-EPDM, the highest dosing of CTBBS before gelation is only $10 \%$. In principle, one CTBBS molecule can only consume one olefinic proton. Therefore, theoretical curves can be drawn as shown in figure 3 . Compared to the theoretical curves, the experimental curves deviate significantly. The ENB-double bond conversion is higher than might have been expected on the basis of stoichiometry, which means many more olefinic protons are consumed than expected. This indicates the fact that side reactions take place during grafting. 


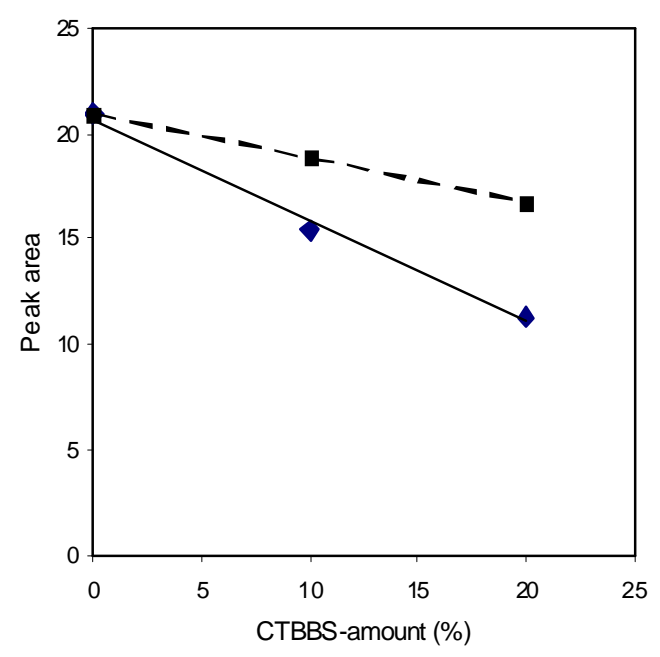

(a)

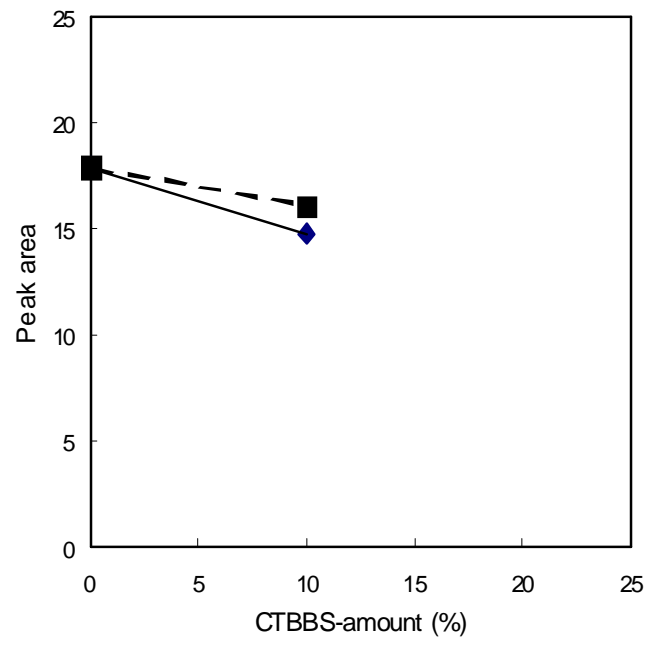

(b)

Figure 3.3 Calibration curves of olefinic proton peak areas versus CTBBS-amount added for ENB-EPDM: (-)experimental; (ロ--- $)$ theoretical; (a): LENB-EPDM; (b): HENBEPDM

Scheme 3.2 shows potential reactions of CTBBS with ENB-EPDM. At the first stage of grafting, a simple addition reaction happens between CTBBS and ENBEPDM. Because of steric resistance effects, the sulphonamidothio-radical covalently bonds to the exocyclic olefinic carbon atom and the chloride radical to the olefinic carbon atom in the ring of ENB. However, this reaction product is not stable and will react further. A chloride ion can be eliminated easily because of the high stability of the carbon cation being formed. Thereafter, the double bond is reformed and hydrochloric acid is released. As the ENB-group is very sensitive to cationic reactions, the presence of hydrochloric acid promotes the gelation of ENB-EPDM via crosslinking. Calcium stearate has been added in order to scavenge acidity and limit cationic side-reactions, but with limited success. Other acid acceptors like amines and $\mathrm{ZnO}$ have also been tried without improvement.

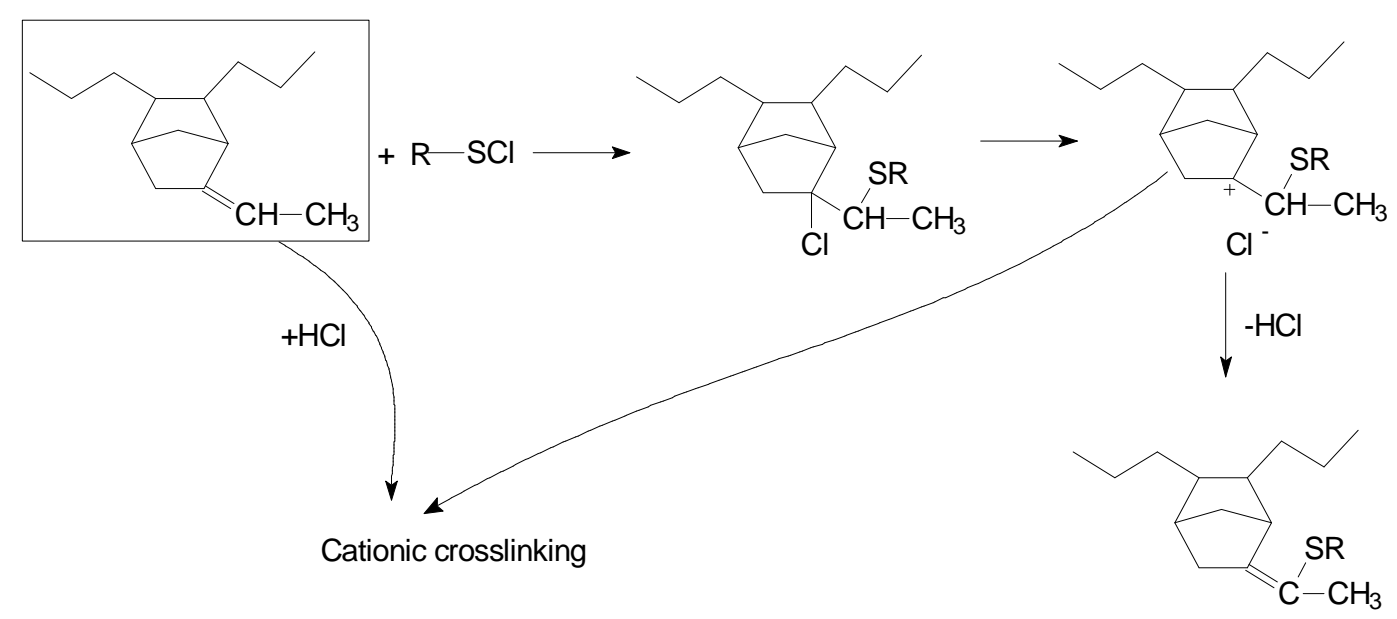

Scheme 3.2 Reactions during CTBBS-grafting onto ENB-EPDM. 
NMR-spectra were recorded at different reaction times. After 4 hours reaction the olefinic proton peak area was stabilized. For ENB-EPDM, four hours is sufficient for the reaction to reach completion.

\subsubsection{HD-EPDM}

Figure 3.4 shows the ATR/IR-spectra of CTBBS-grafted HD-EPDM in comparison with virgin HD-EPDM. The same additional peaks can be seen as for grafted ENB-EPDM. These peaks and their associated functional groups are listed in Table 3.5. The presence of above absorption peaks again indicates that CTBBSfragments are grafted onto HD-EPDM.

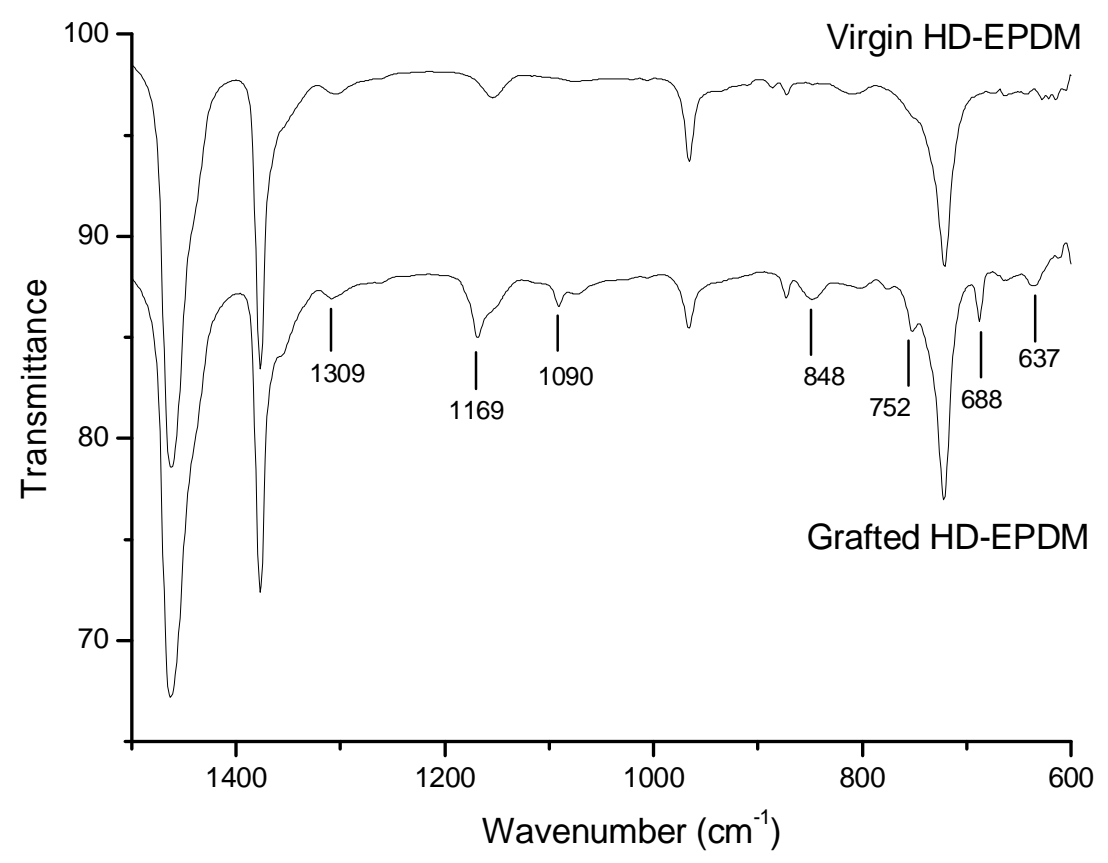

Figure 3.4 ATR/IR-spectra of CTBBS-grafted HD-EPDM in comparison with virgin HD-EPDM.

The NMR-spectra of CTBBS-grafted HD-EPDM and virgin HD-EPDM are shown in figure 3.5. There are three groups of additional peaks. Those at $7.4 \mathrm{ppm}$ to 8ppm can be attributed to the aromatic group of CTBBS, the same as for grafted ENB-EPDM. The peak at $5.4 \mathrm{ppm}$ is due to olefinic protons of the hexadiene group. As soon as grafting occurs, the amount of the olefinic protons decreases. Calibration curves for the area of this peak are shown in figure 3.6. 


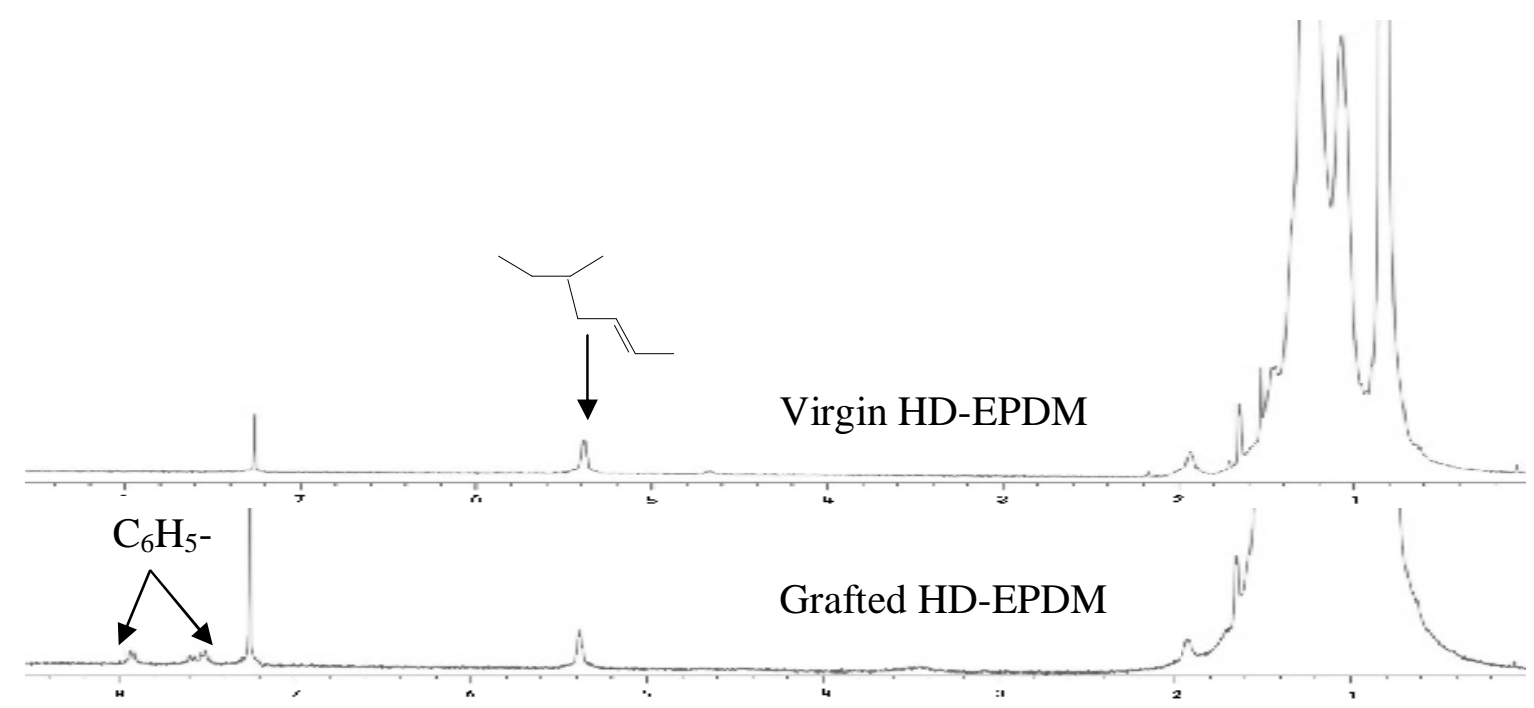

Figure 3.5 NMR-spectra of CTBBS-grafted HD-EPDM in comparison with virgin EPDM.

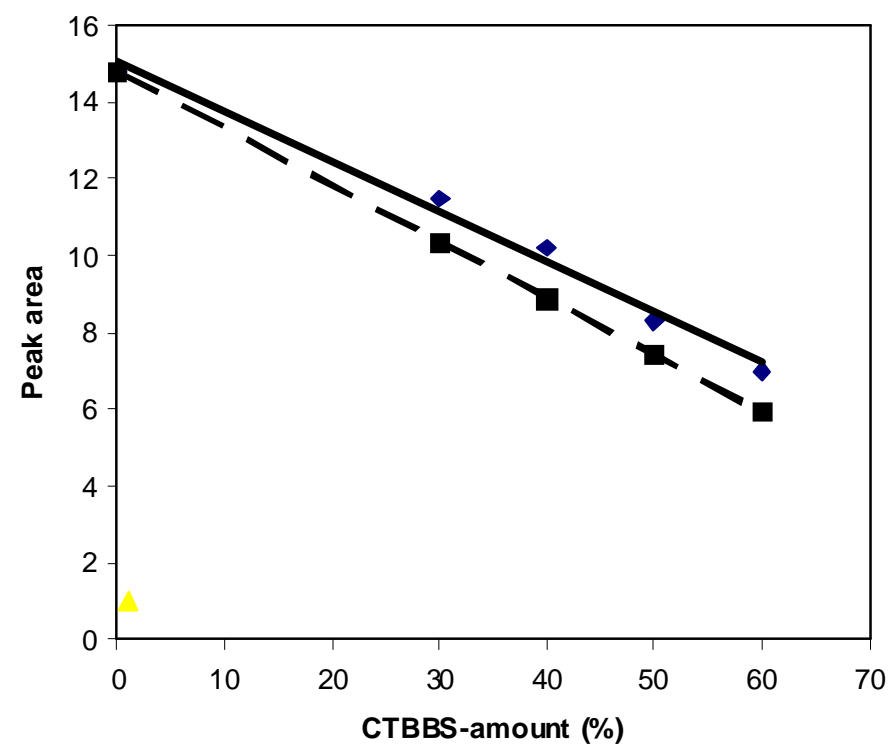

Figure 3.6 Calibration curves of olefinic proton peak areas versus CTBBS-amount added for HD-EPDM: $(-\cdots)$ experimental; ( $----\mathbf{n})$ theoretical.

For HD-EPDM, the accessible CTBBS-amount before gelation occurs is $60 \%$, substantially higher than for ENB-EPDM. Furthermore, the slope of the experimental curve is lower than that of the theoretical one, which means the double bond conversion is less than theoretically expected. It indicates that there are less side reactions taking place compared to ENB-EPDM. By calculation, the double bond conversion of HD-EPDM after CTBBS grafting is $85 \%$ of the theoretical value. Apparently, a different reaction scheme must be proposed to explain the difference between HD-EPDM and ENB-EPDM. 
As shown in scheme 3.3, a simple 1,2-addition reaction will occur between CTBBS and HD-EPDM. The sulphonamidothio-radical covalently bonds to one olefinic carbon atom and the chloride radical bonds to the other. This product is much more stable than that of ENB-EPDM. The elimination of chloride is much more difficult and less hydrochloric acid is formed to initiate cationic crosslinking. Therefore, the CTBBS-amount before geltation for HD-EPDM is much higher than that for ENB-EPDM. Moreover, for HD-EPDM forty-eight hours are needed for the reaction to be complete, much longer than for ENB-EPDM as well.
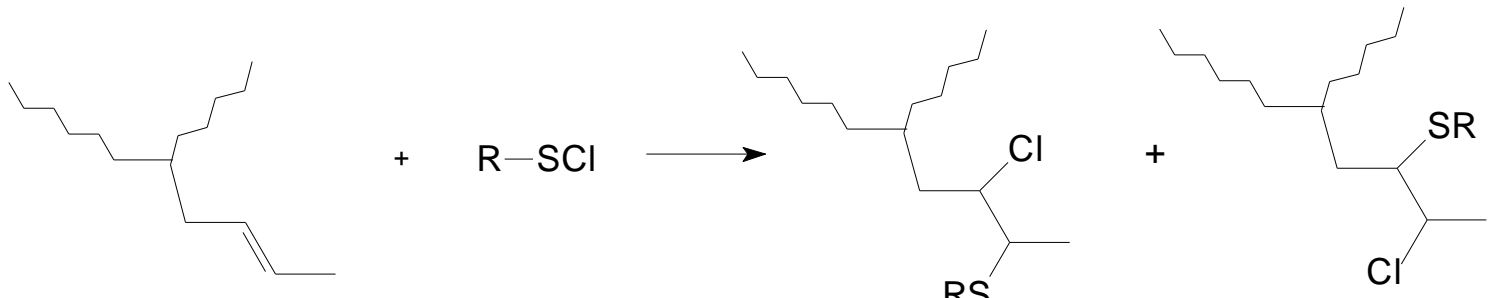

RS

Scheme 3.3 Grafting reaction of CTBBS onto HEX-EPDM.

\subsubsection{DCPD-EPDM}

Despite the fact that the solution of DCPD-EPDM and CTBBS was stirred for longer time and at higher temperature $(80 \square)$ than ENB-EPDM and HD-EPDM, the ATR/IR-spectrum shows no evidence of CTBBS-grafting. This is also confirmed by NMR spectrum, as shown in figure 3.7. The area of the two peaks from olefinic protons remains unchanged after reaction.

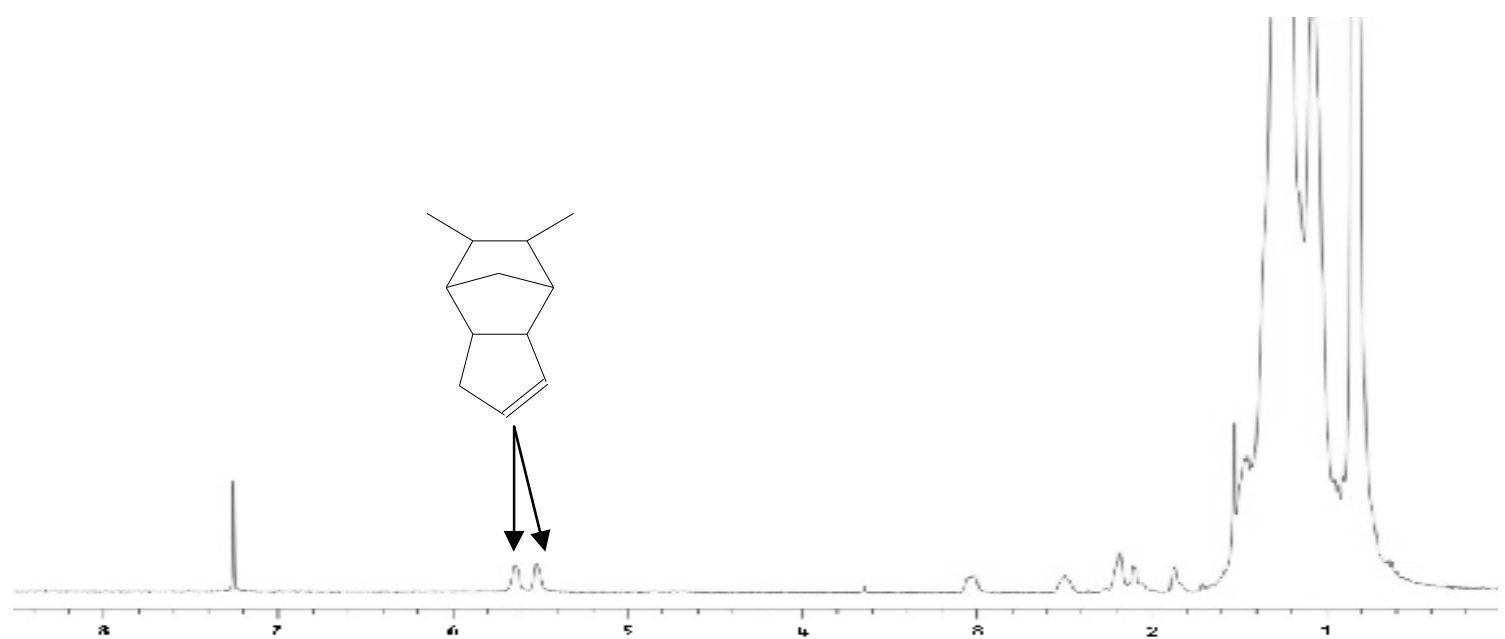

Figure 3.7 NMR-spectra of DCPD-EPDM. 


\subsubsection{Comparison of the various EPDMs}

It is remarkable that grafting happens for ENB-EPDM and HD-EPDM, but not for DCPD-EPDM. Figure 3.8 shows the structure of the three diene monomer types built into EPDM. The different reactivity of the double bond in their structure is the reason for the different behaviors of those three EPDMs during CTBBS-grafting. Considering the difference in reaction speed, the reactivity of the three EPDMs towards grafting varies in the order ENB-EPDM > HD-EPDM > DCPD-EPDM. Unfortunately, the CTBBS-grafting in the case of ENB-EPDM is accompanied by a strong tendency towards gelation due to cationic side reactions. This substantially limits the attainable conversion.

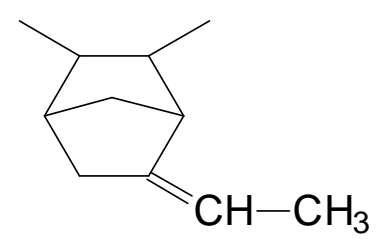

(a)

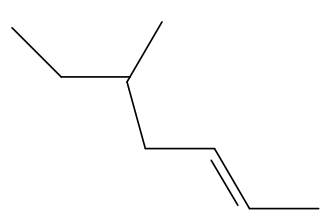

(b)

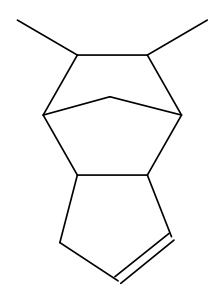

(c)

Figure 3.8 Structures of diene monomers in EPDM: (a) ENB; (b) HD; (c) DCPD.

While HD-EPDM has the highest conversion with CTBBS, it is not commercially available anymore. ENB-EPDM has a too low efficiency, which is not a viable alternative.

\subsubsection{Grafting LENB-EPDM in an internal mixer}

Grafting of LENB-EPDM with CTBBS was also done in an internal mixer with different CTBBS-amounts and at different temperatures in order to minimize gelation. Figure 3.9 shows the torque and temperature curves during mixing for the blend with $20 \%$ equivalent CTBBS. After the addition of CTBBS, the torque first decreases and then rises immediately to its previous value and even a little higher. With temperature increasing continuously, the torque value stays more or less at a constant value, indicating a steady increase in viscosity of the compound. When the CTBBS-amount was increased to $30 \%$, the torque value decreased after CTBBS-addition, and did not rise anymore. The EPDM became grainy, which indicates that gelation happened again. 


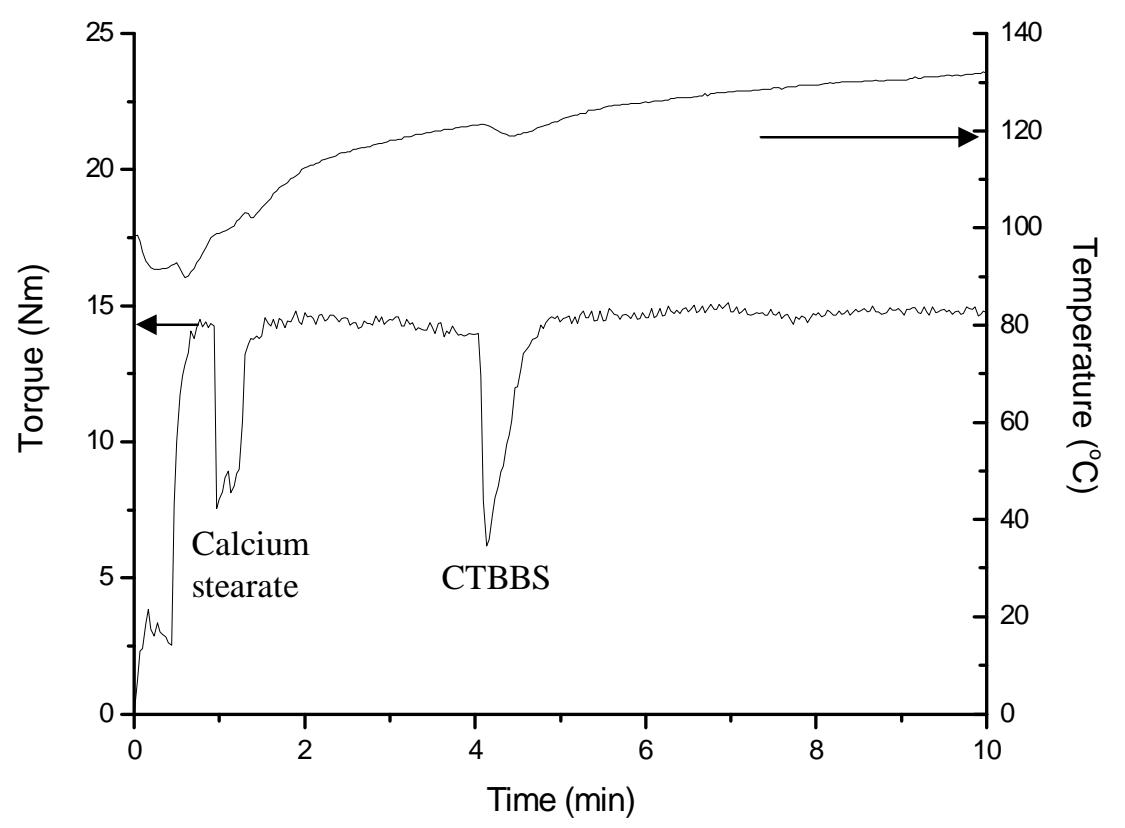

Figure 3.9 Torque and temperature curves during mixing of LENB-EPDM with $20 \%$ CTBBS at $100^{\circ} \mathrm{C}$.

The integration of the area of olefinic protons from NMR-spectrum for the $20 \%$ CTBBS-grafted EPDM is shown in Table 3.6. The value by the internal mixer method is almost the same as by the solution method. $100^{\circ} \mathrm{C}$ and 10 minutes are sufficient for the reaction to reach saturation, because there is no change with longer mixing time or higher temperature. Apparently, grafting in the internal mixer gives similar results as in solution. The problem of gelation still exists.

Table 3.6 Integration area of olefinic hydrogen with 20\% CTBBS.

\begin{tabular}{lc}
\hline \multicolumn{1}{c}{ LENB-EPDMs } & Integration area of olefinic hydrogens \\
\hline Pure LENB-EPDM & 20.9 \\
Internal mixer $100^{\circ} \mathrm{C}(10 \mathrm{~min})$ & 11.6 \\
Internal mixer $100^{\circ} \mathrm{C}(15 \mathrm{~min})$ & 11.6 \\
Internal mixer $120^{\circ} \mathrm{C}(10 \mathrm{~min})$ & 11.9 \\
Solution method & 11.3 \\
\hline
\end{tabular}

\subsubsection{Properties of mixer CTBBS-grafted LENB-EPDM in vulcanised blends with NR/BR}

Figure 3.10 shows the curing curves of NR/BR/EPDM blends according to Table 3.4 with different CTBBS-amounts grafted LENB-EPDM. Compared to 
ungrafted EPDM, blends with grafted EPDM have a longer scorch time due to the amine-effect from CTBBS ${ }^{20,21}$. Grafted EPDM also shows higher torque values than pure EPDM, while with different CTBBS-amounts there is little difference.

Properties for those blends are reported in Table 3.7. Blends with CTBBSgrafted LENB-EPDM show higher moduli than blends with pure LENB-EPDM, which is consistent with the torque values from the curing curves. Tensile strength and elongation at break show limited improvement by applying grafted EPDM into the blends. For different CTBBS-amounts from $10 \%$ to $25 \%$, the differences are limited as well. As for $25 \%$ CTBBS, the EPDM was already partly gelled, higher CTBBSamounts will not help to get better mechanical properties.

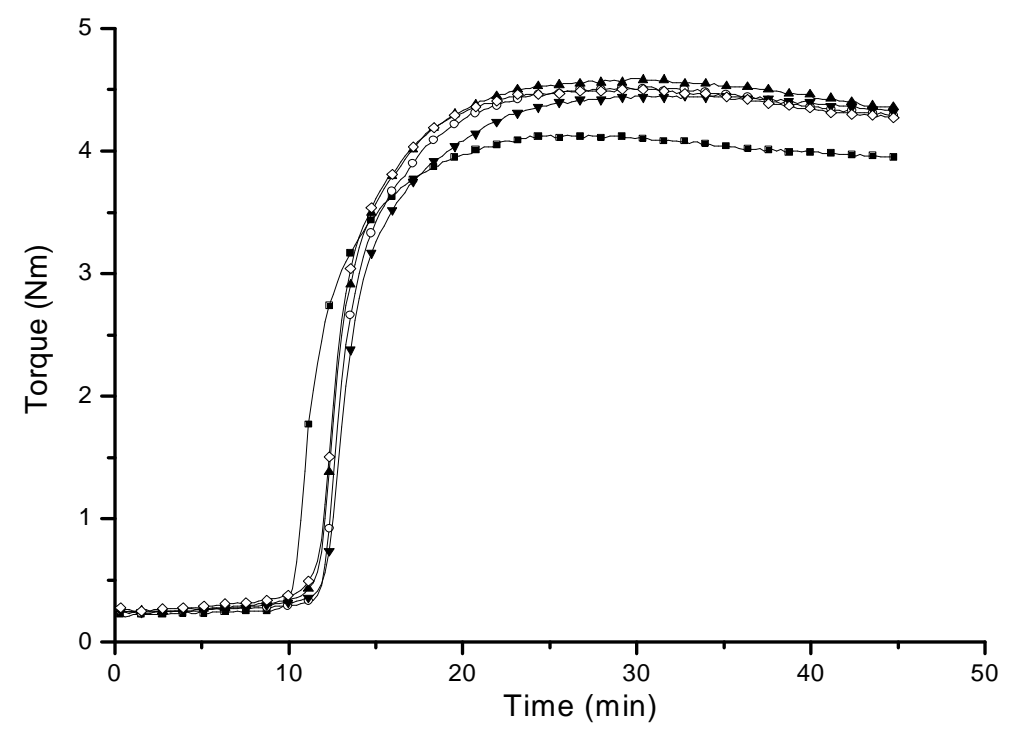

Figure 3.10 RPA curing curve of NR/BR/EPDM blends with different CTBBS amounts grafted LENB-EPDM: (-)0\%; (०)10\%; ( $\mathbf{\Delta}) 15 \% ;(\boldsymbol{\nabla}) 20 \% ;(\diamond) 25 \%$.

Table 3.7 Mechanical properties of NR/BR/EPDM blends with different CTBBS-amounts grafted-EPDM.

\begin{tabular}{lccccc}
\hline & \multicolumn{5}{c}{$\begin{array}{c}\text { CTBBS amounts grafted onto LENB-EPDM relative to } \\
\text { available unsaturation }\end{array}$} \\
\cline { 2 - 6 } Tensile Properties & $0 \%$ & $10 \%$ & $15 \%$ & $20 \%$ & $25 \%$ \\
& 1.43 & 1.48 & 1.54 & 1.52 & 1.53 \\
\hline $100 \%$ Modulus (MPa) & 2.21 & 2.38 & 2.44 & 2.40 & 2.42 \\
$200 \%$ Modulus (MPa) & 3.10 & 3.72 & 3.87 & 3.80 & 3.85 \\
$300 \%$ Modulus (MPa) & 4.3 & 5.0 & 4.8 & 4.9 & 4.7 \\
Tensile Strength (MPa) & 300 & 340 & 310 & 320 & 310 \\
Elongation at Break(\%) & & & & & \\
\hline
\end{tabular}


In Hopper's work $^{15}$, wherein 30phr N-Chlorothio-N-methyl-ptoluenesulphonamide modified HD-EPDM was blended with 70phr NR, tensile properties improved significantly compared with unmodified HD-EPDM/NR blends. Unfortunately, the withdrawal of HD-EPDM from the market does not allow to perform such comparison in the current study anymore.

\subsection{Conclusions}

$\mathrm{N}$-chlorothio-N-butyl-benzenesulphonamide (CTBBS) has a different behavior with respect to grafting on various EPDM-types. The highest grafting efficiency can be reached for HD-EPDM. No grafting is observed for DCPD-EPDM. ENB-EPDM has the highest reactivity, however gelation is observed even at low conversions. The highest grafting level achievable for LENB-EPDM is $20 \%$ relative to the available unsaturation, and for HENB-EPDM, even less.

Both a solution method and an internal mixer method have been tried for the CTBBS-grafting reaction onto ENB-EPDM and show almost the same results. From tensile measurements on vulcanised blends with NR/BR, it must be concluded that ENB-EPDM grafted with $25 \%$ of CTBBS is insufficient to provide significantly improved mechanical properties of the blends compared to historic data on basis of HD-EPDM.

\section{REFERENCES}

1. Waddell, W. H., Rubber Chem. Technol., 71, 590 (1998).

2. Chapman, A. V.; Tinker, A. J., Kautsch. Gummi Kunstst., 56, 533 (2003).

3. Guillaumond, F. X., Revue Generale des Caoutchoucs et Plastiques, 51, 853 (1974).

4. Ignatz-Hoover, F.; To, B. H.; Datta, R. N.; Hoog, A. J. d.; Huntink, N. M.; Talma, A. G., Rubber Chem. Technol., 76, 747 (2003).

5. $\quad$ Callan, J. E.; Hess, W. M.; Scott, C. E., Rubber Chem. Technol., 44, 814 (1971).

6. Kluppel, M.; Schuster, R. H.; J.Schaper, Rubber Chem. Technol., 72, 91 (1999).

7. Ven, P. M. V. d.; Noordermeer, J. W. M., Rubber World, 222, 55 (2000).

8. Zhao, J.; Ghebremeskel, G., Kautsch. Gummi Kunstst., 3, 84 (2001).

9. Woods, M. E.; Davidson, J. A., Rubber Chem. Technol., 49, 112 (1976).

10. Coran, A. Y., Rubber Chem. Technol., 61, 281 (1988).

11. Oliveira, M. G.; Soares, B. G., Macromol. Rapid Commun., 20, 526 (1999).

12. Baranwal, K. C.; Son, P. N., Rubber Chem. Technol., 47, 88 (1974). 
13. Morrissey, R. T. The Meeting of Rubber Division, American Chemical Society, Florida, April, 1971.

14. Hashimoto, K.; Miura, M.; Takagi, S.; Okamoto, H., Int. Polym. Sci. Technol., 3, T84 (1976).

15. Hopper, R. J., Rubber Chem. Technol., 49, 341 (1976).

16. Hopper, R. J.; The Goodyear Tyre \& Rubber Company, U.S. 3,970,133, 1976.

17. Hopper, R. J.; The Goodyear Tyre \& Rubber Company, U.S. 4,820,780, 1989.

18. Hopper, R. J.; Roger, J.; The Goodyear Tyre \& Rubber Company, U.S. 5,756,590, 1998.

19. Chiantore, O.; Cinquina, P.; Guaita, M., European Polym. J., 30, 1043 (1994).

20. Hopper, R. J., Rubber Chem. Technol., 47, 79 (1974).

21. Leib, R. I.; Sullivan, A. B.; Trivette, C. D., Rubber Chem. Technol., 43, 1188 (1970). 


\section{Chapter 4}

\section{Maleic-Anhydride Grafted EPM as Compatibilising Agent in NR/BR/EPDM Blends: Part I. Preparation, Cure Characteristics and Mechanical Properties}

Incorporation of approximately 30phr Ethylene-PropyleneDiene rubber (EPDM) into natural rubber (NR)/butadiene rubber $(\mathrm{BR})$ is a means to achieve non-staining ozone resistance for tyre sidewall applications. However, due to incompatibility of the elastomers and heterogeneous filler distribution in each of the rubber phases, the mechanical properties deteriorate. In the present work, Maleic-Anhydride modified EPM (MAH-EPM) is added as a compatibilising agent between NR/BR and EPDM. The addition of $5 \mathrm{phr}$ of MAH-EPM results in significantly improved tensile and tear strength when compared to a straight NR/BR/EPDM blend. These improvements indicate that MAHEPM does improve the rubber compatibility and the carbon black distribution between the NR/BR and EPDM phases. 


\subsection{Introduction}

There is a great technological interest in the use of blends of various rubbers to achieve tunable enhanced properties. But blending of dissimilar rubbers is severely restricted due to lack of technological compatibility of the component rubbers. Three types of mutual incompatibility can generally exist between dissimilar elastomers: imcompatibility due to viscosity mismatch which prevents or greatly delays the formation of intimate blends ${ }^{1}$; thermodynamic incompatibility, which prevents mixing on the molecular scale; and imcompatibility due to cure rate mismatch².

Blending of Ethylene-propylene-diene rubber (EPDM) with high-diene rubbers attracts lots of interest, as such blends may provide a broader range of applications. EPDM is well known as an ozone-resistant polymer. Therefore, incorporation of EPDM into high-diene rubbers such as natural rubber (NR) and butadiene rubber (BR) is one of the methods to achieve good ozone resistance, instead of using conventional antiozonants which have staining effects and leach out via migration ${ }^{3}$. However, vulcanisates of such elastomer blends are generally poor in both static and dynamic mechnical properties. The main problems associated with the generally poor properties of EPDM/polydiene blends are the significant difference in their unsaturation levels ${ }^{4,5}$. First, EPDM and polydiene rubbers are not miscible on a molecular level due to a viscosity mismatch and thermodynamic incompatibility caused by differences in polarities ${ }^{1}$, resulting in heterogeneous systems. Second, the difference in the amount of unsaturation of each elastomer results in different reactivities for sulphur vulcanisation ${ }^{6}$. Curatives, which are commonly rather polar molecules, generally diffuse more into the highly unsaturated rubbers phases, resulting in differences in concentration between reactants and hence uneven crosslink distribution ${ }^{7,8}$. Carbon black also prefers to migrate into the higher unsaturated polymers, causing a heterogeneous filler distribution in the blends ${ }^{9-11}$.

Previous studies tried to solve these problems by using trans-polyoctylene rubber $(\mathrm{TOR})^{12}$ as a plasticiser to obtain a fine dispersion of EPDM in a NR matrix; by adding polar polymers as polychloroprene ${ }^{13}$, chlorinated polyethylene, and chlorosulfonated polyethylene ${ }^{14}$ as compatibilising agents to improve the affinity of EPDM towards polydiene rubber; by grafting accelerators or accelerator precursors onto EPDM to promote vulcanisation between the EPDM phase and the poly-diene matrix $^{15-20}$. Coran ${ }^{21}$ found that, if the EPDM rubber is functionalised with maleicanhydride or maleic acid, the mechanical properties of vulcanised blends of the functionalised EPDM with NR are greatly improved.

EPDM rubber is particularly known for its superior resistance to ozone and ageing in comparison with natural rubber and common polydiene synthetic rubbers, 
due to its saturated backbone. It has long been recognized that incorporation of 20 40 phr EPDM into conventional tyre sidewall compounds of NR and BR blends is sufficient to "repair" for a loss of ozone resistance due to the omission of the effective but highly staining and discoloring antiozonant $\mathrm{N}$-(1,3-dimethylbutyl)-N'-phenyl-pphenylenediamine (6PPD) ${ }^{9,22-24}$. A mechanism of protection against ozone-induced cracking by the incorporation of essentially saturated polymers such as EPDM rubber, has been proposed by Andrews ${ }^{25}$. It is believed that EPDM is present in particles or regions dispersed in the high-diene rubber phase. These EPDM particles can then interrupt the propagation of micro-cracks in the high diene rubber phase caused by ozone attacking.

In the present work, maleic-anhydride grafted EPM (MAH-EPM) is added as a compatibilising agent to promote the compatibility and bridge the polarity difference between NR/BR and EPDM. The mechanical properties and ozone resistance are reported in this paper as the first part of a series of two.

\subsection{Experimental}

\subsubsection{Materials}

The elastomers selected for the blend were natural rubber (SIR20, Standard Indonesian Rubber), butadiene rubber (Kosyn ${ }^{\circledR}$ KBR01, Korea Kumho Petrochemical Co., Ltd), EPDM rubber containing ethylidene norbornene (ENB) as third monomer (Keltan ${ }^{\circledR}$ 5508, DSM Elastomers B.V., the Netherlands) and three different EPM (DSM Elastomers B.V., the Netherlands), which are EPM (Keltan ${ }^{\circledR} 3200 \mathrm{~A}$ ), maleic anhydride grafted EPM (MAH-EPM) and N-phenyl-p-phenylenediamine (NPPDA) modified MAH-EPM (NPD-EPM). Keltan 5508 contains $4.5 w t \%$ of ethylidene norbornene (ENB) and 70wt\% of ethylene. All three types of EPM have a 50/50wt\% ethylene/propylene ratio. MAH-EPM contains $2.0 \mathrm{wt} \%$ maleic anhydride groups. The structures of MAH-EPM and NPD-EPM are shown in scheme 4.1.

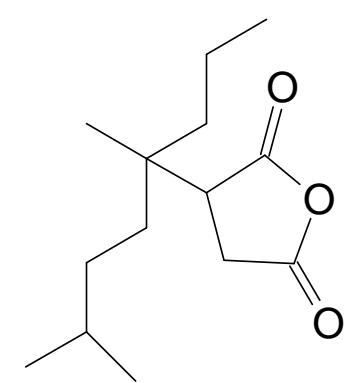

MAH-EPM

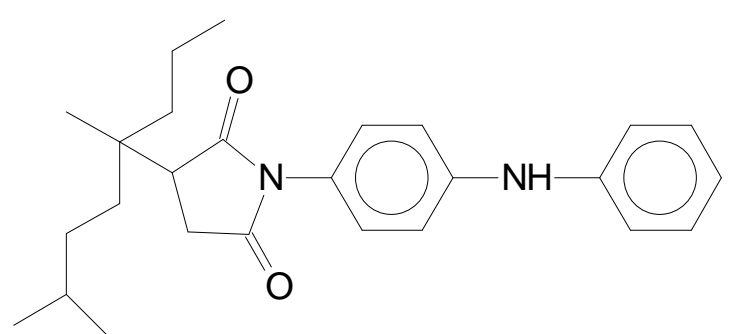

NPD-EPM

Scheme 4.1 Structures of MAH-EPM and NPD-EPM. 
The other compounding ingredients used were extra pure grade zinc oxide (Merck, Germany), finely divided sulphur (Merck, Germany), 95\% pure stearic acid (Aldrich, Germany), poly (2,2,4-trimethyl-1,2-dihydroquinoline) or TMQ (Flexsys B.V., the Netherlands) and N-cyclohexyl-2-benzothiazolesulfenamide (CBS) or Santocure ${ }^{\circledR}$ (Flexsys B.V., the Netherlands), N-(1,3-dimethylbutyl)-N'-phenyl-p-phenylenediamine (Santoflex ${ }^{\circledR}$ 6PPD, Flexsys B.V., the Netherlands). High abrasion furnace black (HAF-N330, Cabot Corporation) and naphthenic oil Sunthene ${ }^{\circledR} 4240$ (Sun Petroleum Products Co., Ltd.) were used to prepare the masterbatches in the case of filled blends.

\subsubsection{Preparation of blend compounds}

Table 4.1 gives the formulations to study the influence of the amount of $\mathrm{MAH}$ EPM on the properties of non-reinforced blends. The amount of MAH-EPM was varied from 0 to $10 \mathrm{phr}$. The total amount of EPDM plus MAH-EPM was fixed at $30 \mathrm{phr}$.

Table 4.1 Formulations of gum blends with different MAH-EPM amounts (phr).

\begin{tabular}{l|ccccc}
\hline \multicolumn{1}{c|}{ Component } & MAH-0 & MAH-3 & MAH-5 & MAH-7 & MAH-10 \\
\hline NR & 35 & 35 & 35 & 35 & 35 \\
BR & 35 & 35 & 35 & 35 & 35 \\
EPDM & 30 & 27 & 25 & 23 & 20 \\
MAH-EPM & 0 & 3 & 5 & 7 & 10 \\
ZnO & 4 & 4 & 4 & 4 & 4 \\
Stearic acid & 2 & 2 & 2 & 2 & 2 \\
TMQ & 1 & 1 & 1 & 1 & 1 \\
CBS & 2 & 2 & 2 & 2 & 2 \\
Sulphur & 2.5 & 2.5 & 2.5 & 2.5 & 2.5 \\
\hline Total weight & & & 111.5 & & \\
\hline
\end{tabular}

Based on an optimum of 5phr MAH-EPM, a comparison was made between the effect of no EPM, straight EPM, MAH-EPM and NPD-EPM. The formulations of these compounds are given in Table 4.2, divided in two groups: gum and HAF-filled. In each group, Ref- 0 is a reference formulated according to the common industry recipe for tyre sidewalls, containing only NR and BR. Ref-1 is another reference: a straight NR/BR/EPDM blend. EPM-5, MAH-5 and NPD-5 contain 5phr of the different types of EPM as compatibilising agents for comparison. 
Table 4.2 Formulations of NR/BR/EPDM/EPM compounds: gum stocks and black reinforced (phr).

\begin{tabular}{|c|c|c|c|c|c|c|c|c|c|c|}
\hline \multirow{2}{*}{ Component } & \multicolumn{5}{|c|}{ Gum } & \multicolumn{5}{|c|}{ HAF-filled } \\
\hline & Ref-0 & Ref-1 & EPM-5 & MAH-5 & NPD-5 & Ref-0 & Ref-1 & EPM-5 & $\mathrm{MAH}-5$ & NPD-5 \\
\hline NR & 50 & 35 & 35 & 35 & 35 & 50 & 35 & 35 & 35 & 35 \\
\hline $\mathrm{BR}$ & 50 & 35 & 35 & 35 & 35 & 50 & 35 & 35 & 35 & 35 \\
\hline EPDM & 0 & 30 & 25 & 25 & 25 & 0 & 30 & 25 & 25 & 25 \\
\hline EPM & 0 & 0 & 5 & 0 & 0 & 0 & 0 & 5 & 0 & 0 \\
\hline MAH-EPM & 0 & 0 & 0 & 5 & 0 & 0 & 0 & 0 & 5 & 0 \\
\hline NPD-EPM & 0 & 0 & 0 & 0 & 5 & 0 & 0 & 0 & 0 & 5 \\
\hline HAF N330 & 0 & 0 & 0 & 0 & 0 & 50 & 50 & 50 & 50 & 50 \\
\hline $\begin{array}{c}\text { Sunthene } \\
4240\end{array}$ & 0 & 0 & 0 & 0 & 0 & 10 & 10 & 10 & 10 & 10 \\
\hline ZnO & 4 & 4 & 4 & 4 & 4 & 4 & 4 & 4 & 4 & 4 \\
\hline $\begin{array}{c}\text { Stearic } \\
\text { acid }\end{array}$ & 2 & 2 & 2 & 2 & 2 & 2 & 2 & 2 & 2 & 2 \\
\hline TMQ & 1 & 1 & 1 & 1 & 1 & 1 & 1 & 1 & 1 & 1 \\
\hline 6PPD & 2 & 0 & 0 & 0 & 0 & 2 & 0 & 0 & 0 & 0 \\
\hline CBS & 2 & 2 & 2 & 2 & 2 & 2 & 2 & 2 & 2 & 2 \\
\hline Sulphur & 2.5 & 2.5 & 2.5 & 2.5 & 2.5 & 2.5 & 2.5 & 2.5 & 2.5 & 2.5 \\
\hline $\begin{array}{c}\text { Total } \\
\text { weight }\end{array}$ & 113.5 & 111.5 & 111.5 & 111.5 & 111.5 & 173.5 & 171.5 & 171.5 & 171.5 & 171.5 \\
\hline
\end{tabular}

The compounds were prepared in a Brabender Plasticorder 3505 mixer having a mixing chamber volume of $370 \mathrm{~cm}^{3}$. The mixer was operated at a rotor speed of $80 \mathrm{rpm}$, fill factor of 0.7 and an initial temperature of $50^{\circ} \mathrm{C}$. NR was first masticated for 2 minutes, then BR and EPDM/EPM were added and mixed for 1 minute. $\mathrm{ZnO}$, stearic acid and $\mathrm{TMQ}$ were subsequently added and the mixing was continued for another 2 minutes. The final compound temperature before dumping from the mixer was in the range of $110-115^{\circ} \mathrm{C}$. CBS and sulphur were added into the compounds on a two-roll mill.

In the case of carbon black filled compounds, the same conditions were applied. NR was first masticated for 2 minutes, then BR and EPDM/EPM were added and mixed for 1 minute. After that, HAF black, oil and other additives were added and mixing continued for another 2 minutes. CBS and sulphur were added to the batches on the two-roll mill.

\subsubsection{Vulcanisation}

The compounds were tested for their cure characteristics using a RPA 2000 dynamic mechanical cure meter of Alpha Technologies at $140^{\circ} \mathrm{C}, 0.833 \mathrm{~Hz}$ and 0.2 degree strain, according to ISO 6502. The optimum cure time: $t_{c, 90}$, of the fully compounded blends was determined as the time needed to reach $90 \%$ of the 
maximum torque in the RPA 2000. The blends were then vulcanised for $t_{c, 90}$ in a Wickert WLP1600 laboratory compression press at $140^{\circ} \mathrm{C}$ and 100 bar, into $2 \mathrm{~mm}$ thick sheets.

\subsubsection{Tensile and trouser tear tests}

Type 2 dumb-bell test pieces were die-cut from the compression-molded sheets and tensile tests were carried out according to ISO 37, with a Zwick tensile tester Model Z 1.0/TH1S at a constant crosshead speed of $500 \mathrm{~mm} / \mathrm{min}$.

Trouser tear test specimens of $100 \mathrm{~mm} \times 7.5 \mathrm{~mm}$ with a cut of depth $40 \mathrm{~mm}$ were die-cut and the tear tests were performed according to ISO 34 Method A, with the Zwick tensile tester at a constant crosshead speed of $100 \mathrm{~mm} / \mathrm{min}$.

\subsubsection{Flex fatigue to failure tests}

Fatigue-to-failure tests were carried out in two ways. The first was using a Monsanto fatigue-to-failure tester with fixed extension ratio according to ASTM D 4482-99. The extended length of the specimen relative to the unextended length was 2.0. The number of stress-strain cycles required to cause failure of the dumbbell specimen, as indicated by complete rupture, was recorded. 12 Test specimens of each sample were tested and the median value of the number of cycles required to cause failure was recorded. The second was to determine the fatigue life at constant strain energy. To measure the strain energy, a cured fatigue specimen was first conditioned by cycling it 30 times to $140 \%$ extension. Using ASTM procedure D-412, a stress-strain curve was generated from which the amount of force per unit area for $10 \%$ to $140 \%$ extension in $10 \%$ increments was recorded. From these data, three extension ratios around a specified strain energy were selected for each sample and with that the fatigue life was determined. The fatigue life at constant strain energy could then be calculated with the following equation: eq 4.1 .

$$
\text { In (Fatigue Life) }=A+B[\ln (\text { Strain Energy })]
$$

The specified strain energy was 1.2MPa for gum blends, and 1.8MPa for HAFfilled blends.

\subsubsection{Static and dynamic ozone testing}

The ozone resistance of the blends was tested according to ISO 1431-1 in an Argentox ozone test cabinet (Argentox Ozone Technology $\mathrm{GmbH}$, Germany) under 
both static and dynamic conditions. The test specimens were $20 \mathrm{~mm}$ in width and $2 \mathrm{~mm}$ in thickness with modified ends, as shown in figure 4.1 .

For the static ozone test, the specimens were clamped between grips, elongated to $20 \%$ and tested under the following conditions: temperature $40 \pm 2^{\circ} \mathrm{C}$; relative humidity $50 \pm 5 \%$; ozone concentration $50 \pm 5 \mathrm{pphm}$ for the first 96 hours, and $100 \pm 5$ pphm for the next 96 hours.

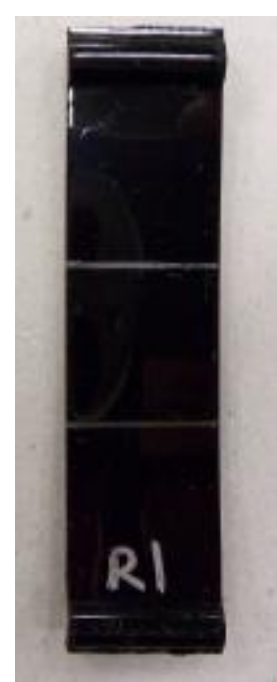

(a)

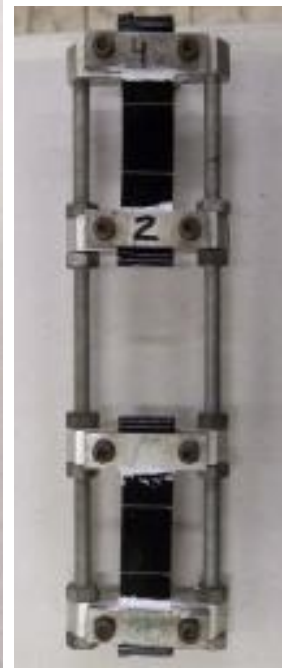

(b)

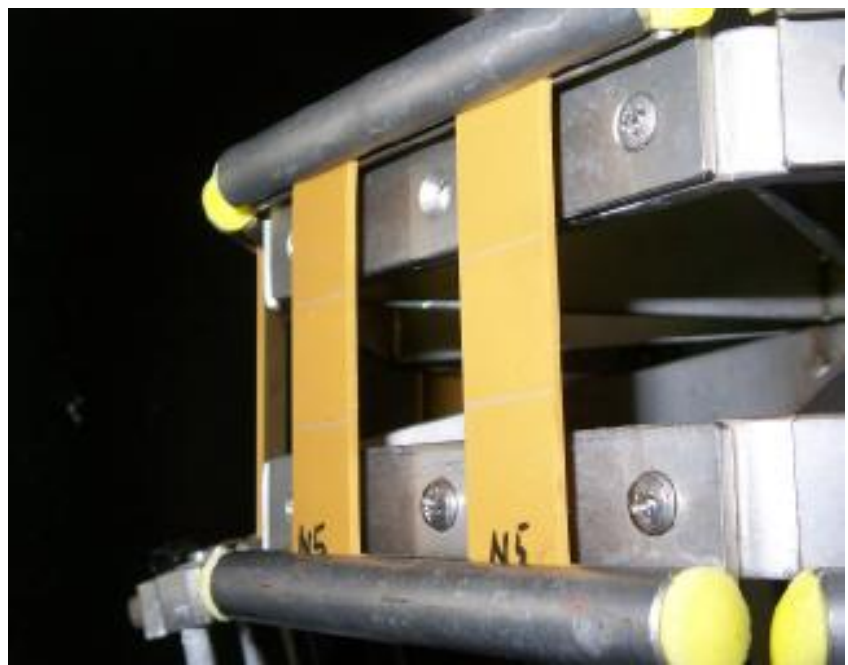

(c)

Figure 4.1 Test specimen (a) and test configuration in ozone cabinet: static condition (b), dynamic condition (c).

For the dynamic ozone test, the test specimens were hanged and elongated using a special-built sample holder (ERT B.V., the Netherlands) in order to prevent an excessive stress concentration and hence breakages at the grips during the dynamic ozone test. The test specimens were attached to the sample holder consisting of a stationary part (top) at a fixed position and a moving part (bottom) which is driven to travel to a specific elongation.

The test is normally carried out at an ozone concentration of $50 \pm 5 p p h m$. In the present study, after the first dynamic test at $50 \pm 5$ pphm ozone concentration and $20 \%$ elongation for 96 hours, no compound showed cracks on the surface. Further test-runs on the same test specimens were then carried out consecutively in order to be able to differentiate between each compound. The dynamic test conditions are shown in Table 4.3. 
Table 4.3 Dynamic ozone test conditions

\begin{tabular}{lccc}
\hline \multicolumn{1}{c}{ Condition } & $1^{\text {st }}$ Run & $2^{\text {nd }}$ Run & $3^{\text {rd }}$ Run \\
\hline Ozone conc. $(\mathrm{pphm})$ & $50 \pm 5$ & $100 \pm 5$ & $100 \pm 5$ \\
Elongation $(\%)$ & 20 & 20 & 30 \\
Temperature $\left({ }^{\circ} \mathrm{C}\right)$ & $40 \pm 2$ & $40 \pm 2$ & $40 \pm 2$ \\
Relative humidity $(\%)$ & $50 \pm 5$ & $50 \pm 5$ & $50 \pm 5$ \\
Frequency $(\mathrm{Hz})$ & 0.5 & 0.5 & 0.5 \\
Time $(\mathrm{h})$ & 96 & 48 & 48 \\
\hline
\end{tabular}

\subsection{Results and Discussion}

\subsubsection{Influence of MAH-EPM amount on the gum blend properties}

Figure 4.2 shows the tensile properties of the gum blends with different MAHEPM amounts. The modulus $100 \%$ increases slightly and the modulus $300 \%$ more clearly when MAH-EPM is added, compared to the straight NR/BR/EPDM blend without MAH-EPM. The tensile strength and elongation at break for the blend with 3phr MAH-EPM is still the same as for the blend without MAH-EPM. With increased amounts of MAH-EPM to 5phr and 7phr, a significant improvement can be observed, especially for the tensile strength. However, with the amount of MAH-EPM increased to $10 \mathrm{phr}$, the tensile strength and the elongation at break decrease again. That is because no co-cure can be obtained between the MAH-EPM and the other types of rubber, as it has a completely saturated structure. This loss of co-cure has a negative influence on the final properties. For carbon black filled compounds, a similar trend could be found.

It is clear that 3phr of MAH-EPM is insufficient to provide improved tensile properties for the blend, while 10phr of MAH-EPM is too much and even reduces the properties. Therefore, $5 \mathrm{phr}$ to $7 \mathrm{phr}$ is the proper amount to improve the properties of the blends. In the continuation of this study, 5 phr MAH-EPM was always used. 


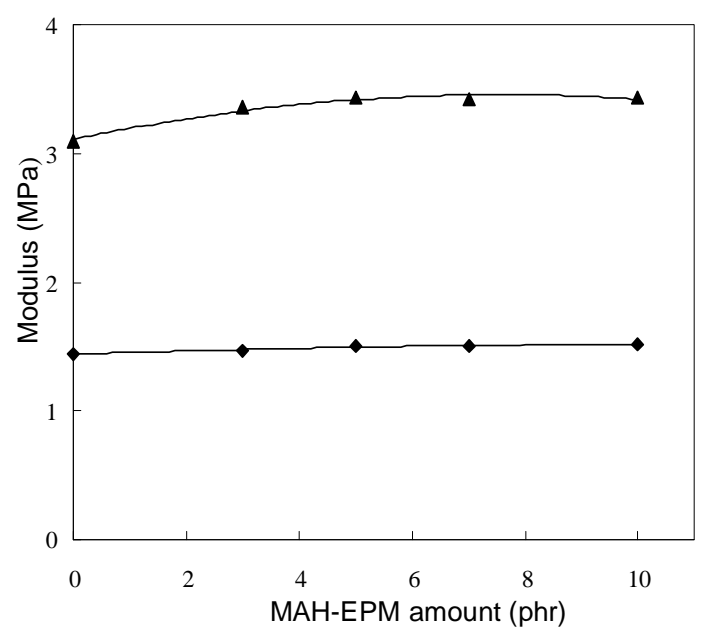

(a)

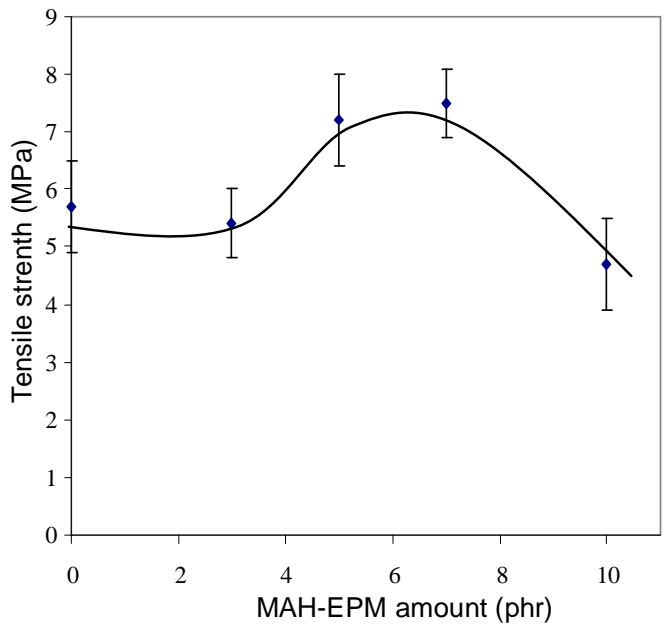

(b)

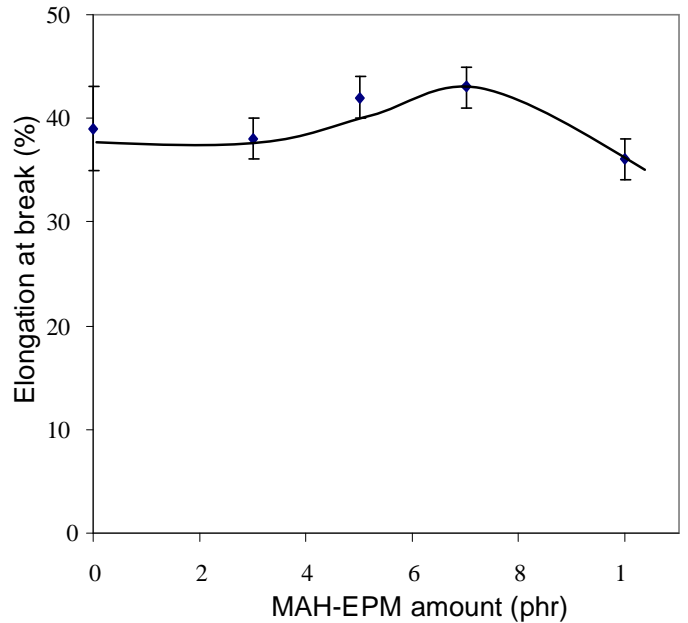

(c)

Figure 4.2 (a): ( $\square \square \square): 100 \%$ modulus; ( $\triangle-\Delta$ ):300\% modulus; (b): Tensile strength; (c): Elongation at break of the blends with different MAH-EPM amounts.

\subsubsection{Influence of 5phr EPM and grafted EPMs on the blend properties: gum stocks and carbon black reinforced}

\subsubsection{Cure Characteristics}

Figure 4.3 shows cure curves of the gum blends and figure 4.4 of the HAFfilled blends with the formulations as shown in Table 4.2. The addition of EPDM to the NR/BR blends increases both scorch time and cure time. For Ref-0 without EPDM, reversion can be observed as opposed to the EPDM-containing blends. EPM-5 has a longer scorch time and lower maximum torque than Ref-1, in both the gum and HAF-filled cases. This can be attributed to the saturated structure of EPM; it does not participate in the curing process, which slows down the cure and decreases the degree of co-cure. The NR/BR and MAH-5 gum blends both show a higher 
maximum torque value than the others. In case of the HAF-filled blends, the maximum torque of MAH-5 is even substantially higher than for Ref-0. MAH-EPM still has a saturated structure, while it combines a longer scorch time with a much higher maximum torque, even higher than that of Ref- 1 . The retarding effect of the cure is due to the maleic anhydride group, as it is well known that acids and anhydrides can be used as retarders for the vulcanisation of rubber. In spite of this retardation, the higher maximum torque indicates the formation of additional crosslinks. This must be due to the effect of the maleic anhydride group, as shown by the fact that the addition of NPD-EPM to the NR/BR/EPDM blend does not give a significant difference in the cure curve due to the capping of the anhydride functionality with the amine. These results are in agreement with the observation of Coran $^{21}$, who interpreted the extra cure of maleic anhydride modified EPDM as the result of ionic crosslinks formed with $\mathrm{ZnO}$ in the compound.

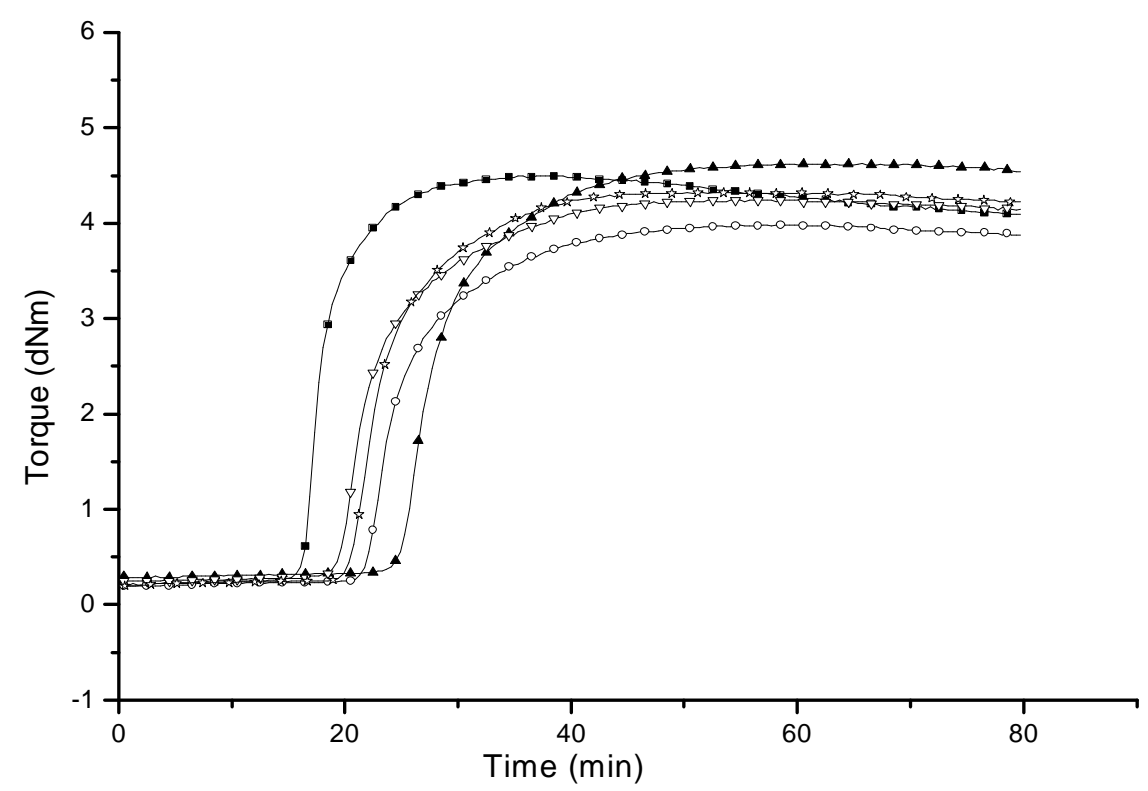

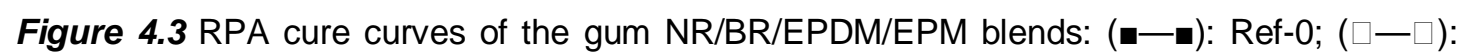

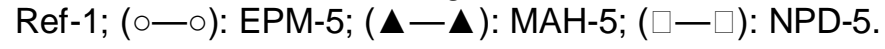




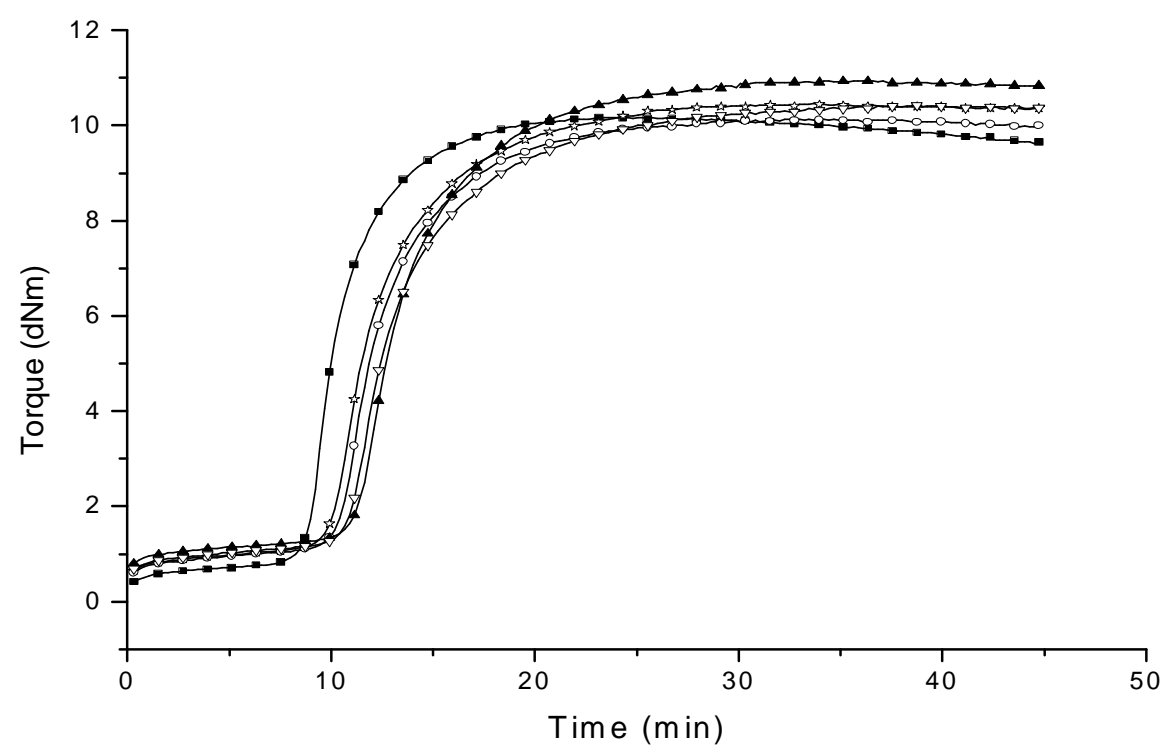

Figure 4.4 RPA cure curves of the HAF-filled NR/BR/EPDM/EPM blends: ( - - ): Ref-0; ( $\square-$

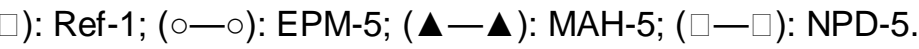

\subsubsection{Tensile and tear properties}

Figure 4.5 and 4.6 show representative stress-strain curves for the gum and HAF-filled blends, respectively. It is clear that both the gum- and HAF-filled-Ref-1 compounds show much poorer tensile properties than Ref-0, commonly blamed on viscosity mismatch, thermodynamic incompatibility, cure incompatibility and inhomogeneous filler distribution. EPM-5 shows even worse tensile properties compared to Ref-1, for the gum blend as well as for HAF-filled blend. The addition of MAH-EPM does improve the tensile properties though compared to the straight NR/BR/EPDM Ref- 1 blends in both the gum and HAF-filled cases. Again, the addition of NPD-EPM does not improve and even damages the stress-strain properties for the gum blend. Also no significant changes for the filled blend were achieved relative to Ref-1. 


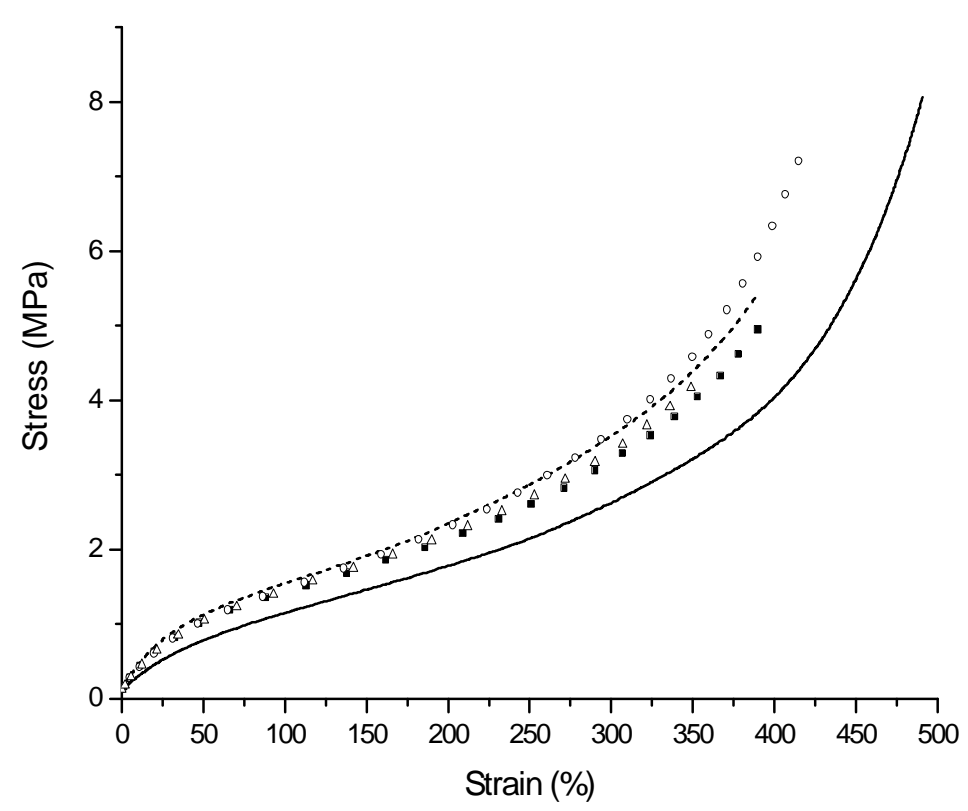

Figure 4.5 Tensile stress-strain curves for the gum NR/BR/EPDM/EPM blends: (一): Ref-0;

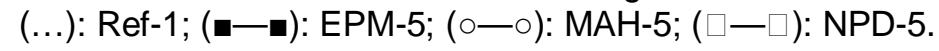

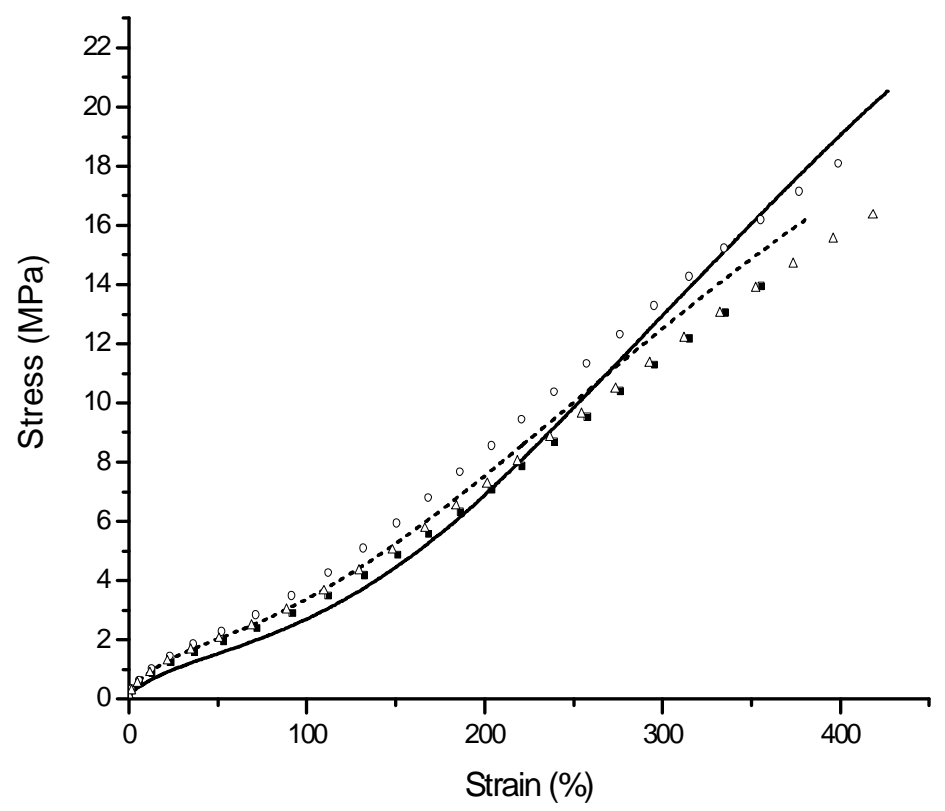

Figure 4.6 Tensile stress-strain curves for the HAF-filled NR/BR/EPDM/EPM blends: ( - ):

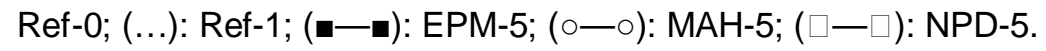

The characteristics data of the tensile properties are summarized in figure 4.7, 4.8 and 4.9. Figure 4.7 shows the tensile moduli at $100 \%$ and $300 \%$ elongation for both the gum and HAF-filled blends. All EPDM containing gum-blends exhibit some higher modulus than the gum NR/BR blend at both $100 \%$ and $300 \%$ strain. For the filled-blends, Ref-0 still has a lower modulus at 100\% than the EPDM containing blends. However, the modulus at $300 \%$ of Ref- 0 is now higher than Ref- 1 , EPM-5 and NPD-5 and more or less the same as MAH-5. In both the gum and HAF-filled cases, EPM-5 has a lower modulus than Ref-1, while MAH-5 has a higher than Ref- 
1; all consistent with the maximum torque already observed in the curing curves. The increased modulus relative to Ref- 1 with the addition of MAH-EPM again indicates effects coming from the maleic anhydride groups, like additional ionic crosslinking in the presence of $\mathrm{ZnO}^{21}$. Gum-NPD-5 and HAF-filled-NPD-5 show similar moduli as the EPM- 5 blends, again indicating that the capping of the anhydride group by the amine prevents the extra ionic crosslinking with $\mathrm{ZnO}$.

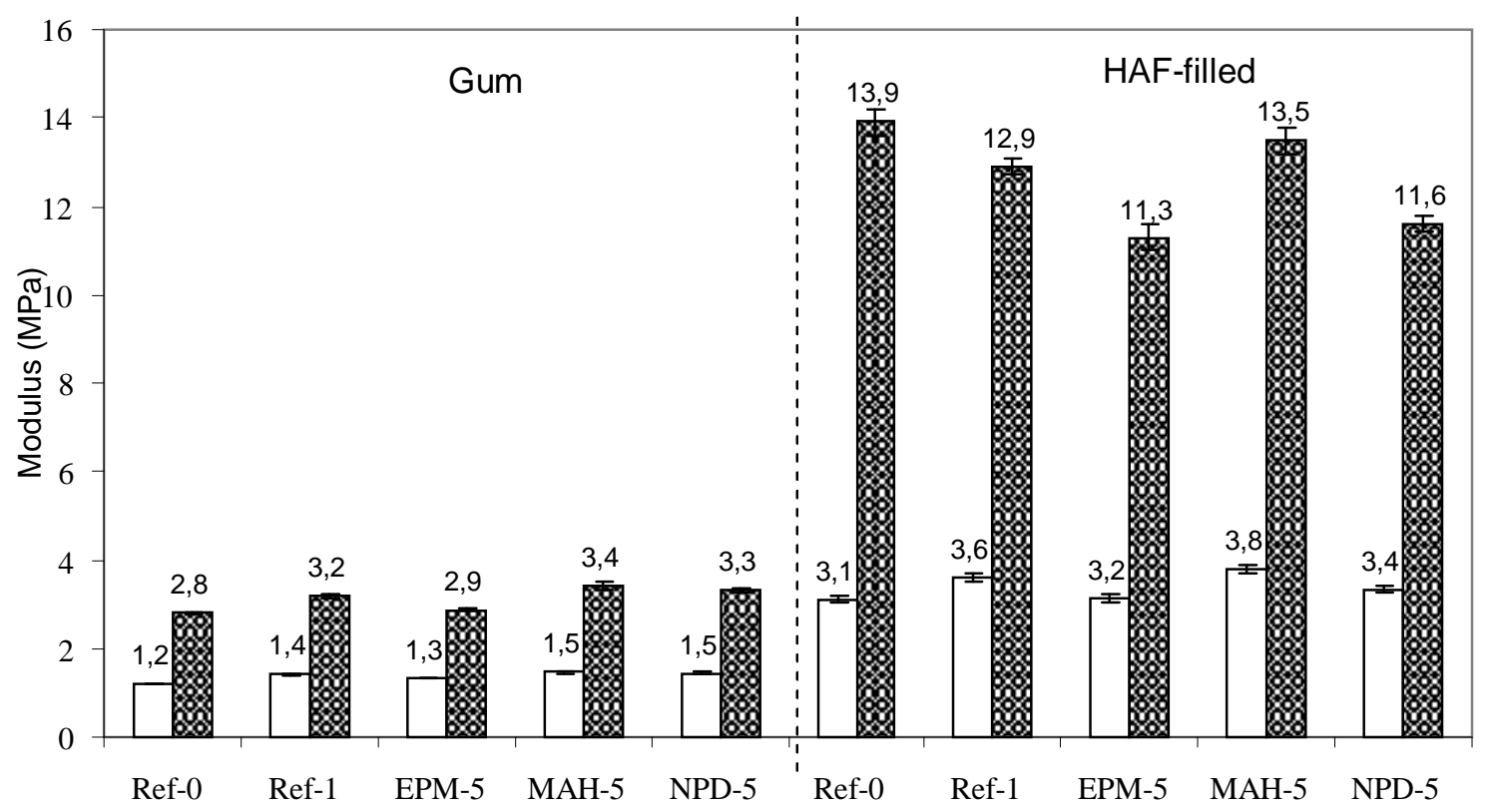

Figure 4.7 Modulus at $100 \%$ and $300 \%$ strain of the blends:

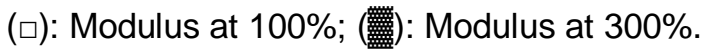

The tensile strengths of the gum and HAF-filled blends are shown in figure 4.8. Ref-1 already has a much lower tensile strength than Ref-0, in both the gum and filled cases. The addition of EPM decreases the tensile strength even further. Nor does NPD-EPM improve the tensile strength. However, with MAH-EPM, a significant improvement in the tensile strengths can be observed compared to the other EPDM containing blends. The tensile strength of $\mathrm{MAH}-5$ is even quite close to that of the pure NR/BR blend. 


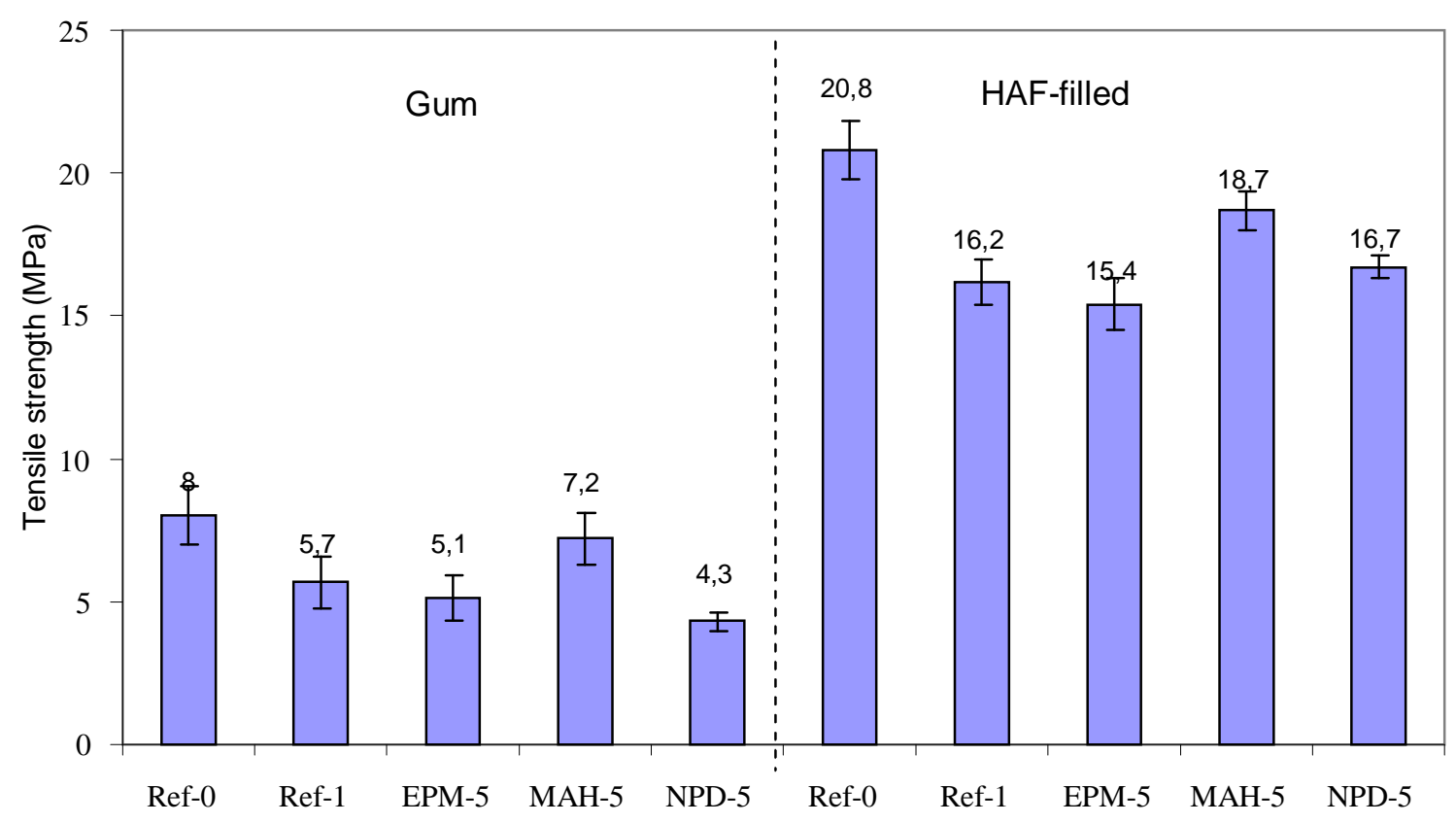

Figure 4.8 Tensile strength of the blends.

For carbon black reinforced compounds, a reinforcement parameter can be defined as the following equation. This reinforcement parameter plays an important role in tyre performance, because it is indicative for the rolling resistance of tyres.

$$
\text { Reinforcement parameter }(\mathrm{RP})=\frac{\text { Modulus at } 300 \%}{\text { Modulus at } 100 \%}
$$

Although the addition of MAH-EPM to the NR/BR/EPDM blends improves their tensile strength significantly and the values are quite close to that of the NR/BR blends, the reinforcement parameter of $\mathrm{MAH}-5$ is still much lower than that of Ref- 0 : 3.6 vs. 4.6. It indicates that the reinforcement effect of the carbon black in the maleicanhydride containing compound is still not on the same level as for the sidewall formulation: Ref-0.

Figure 4.9 shows the elongation at break of the gum and HAF-filled blends. The addition of $30 \mathrm{phr}$ EPDM or the 25/5 EPDM/EPM mixture to the NR/BR recipe results in a decrease of elongation at break: for the gum as well as for the HAF-filled blends. The addition of maleic-anhydride modified EPM partially restores the loss in the gumcase, and practically fully in the black-filled compounds. An interesting case is the use of NPD-EPM, which similar to the tensile strength data, gives the poorest elongation at break for the gum, however in case of the HAF-filled blends equals the elongation values of Ref- 0 . This can be taken again as an indication that NPD-EPM does not increase the compatibility between the different rubber phases in the gum 
blends, while it does lead to some better carbon black distribution for the filled blend, as the amine group has some affinity to the carbon black.

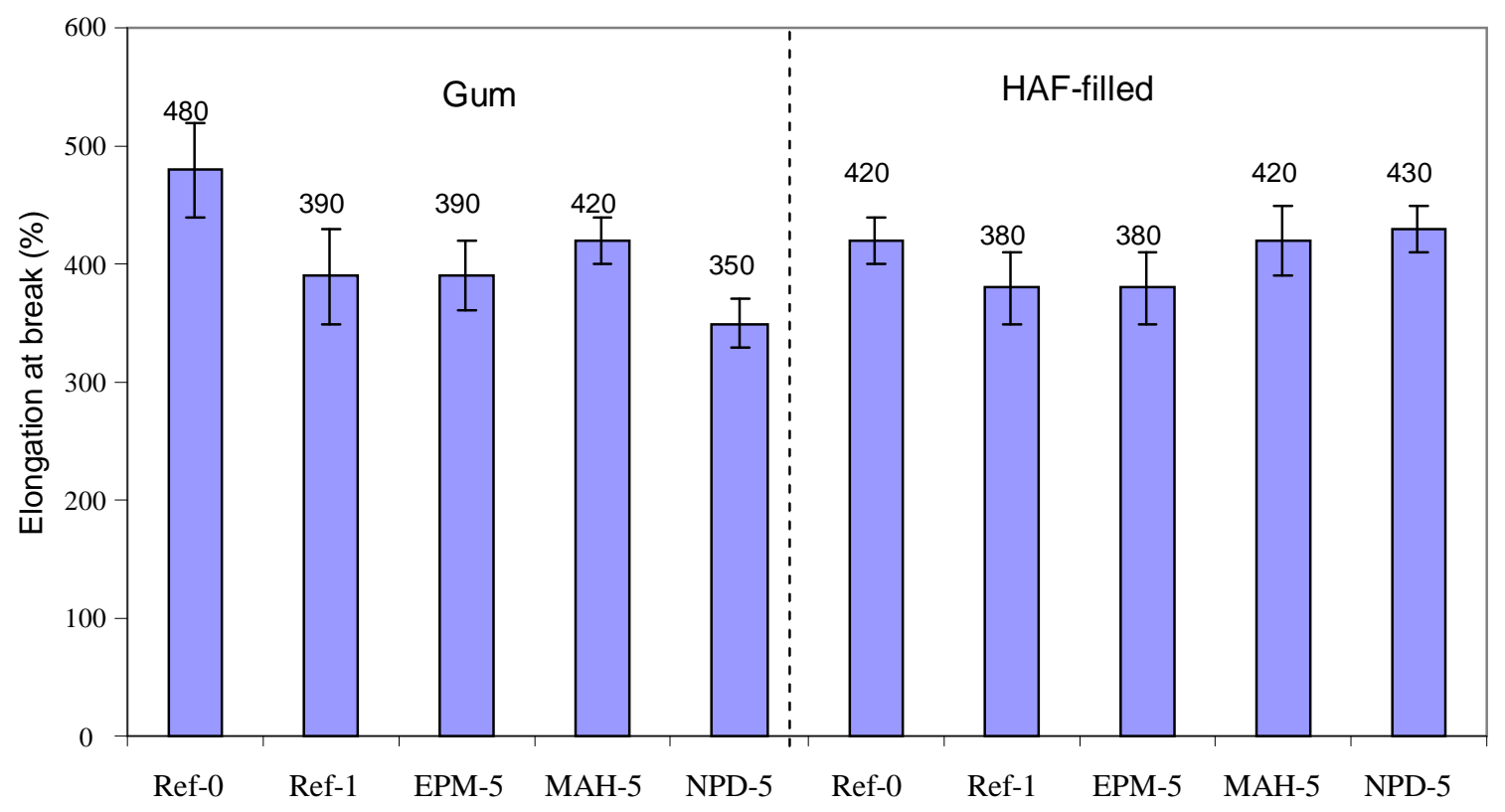

Figure 4.9 Elongation at break of the blends.

The trouser tear strength of gum and HAF-filled blend vulcanisates are shown in figure 4.10. A similar trend can be observed for the tear strength as for the tensile strength. The NR/BR blends exhibit the highest tear strengths. EPM- 5 and NPD-5 do not show an improvement as compared to Ref-1; MAH-5 shows the highest tear strength of all the EPDM containing blends, coming close to Ref- 0 for the black-filled case. The higher tear strength as well as the higher tensile strength of such $\mathrm{NR} / \mathrm{BR} / \mathrm{EPDM} / \mathrm{MAH}-\mathrm{EPM}$ blend vulcanisates, apart from the extra ionic crosslinking, must be the result of a better homogeneity of either crosslink distribution or carbon black distribution or both. Further, a better dispersion of EPDM particles inside the $\mathrm{NR} / \mathrm{BR}$ matrix impedes the tear propagation and hence increases tearing energy or tear strength.

All these improvements suggest that MAH-EPM acts as a compatibilising agent between NR/BR and EPDM. Other effects, such as additional crosslinking also account for improvement. The mechanisms involved will be detailed in the next part of this series: Chapter 5. 


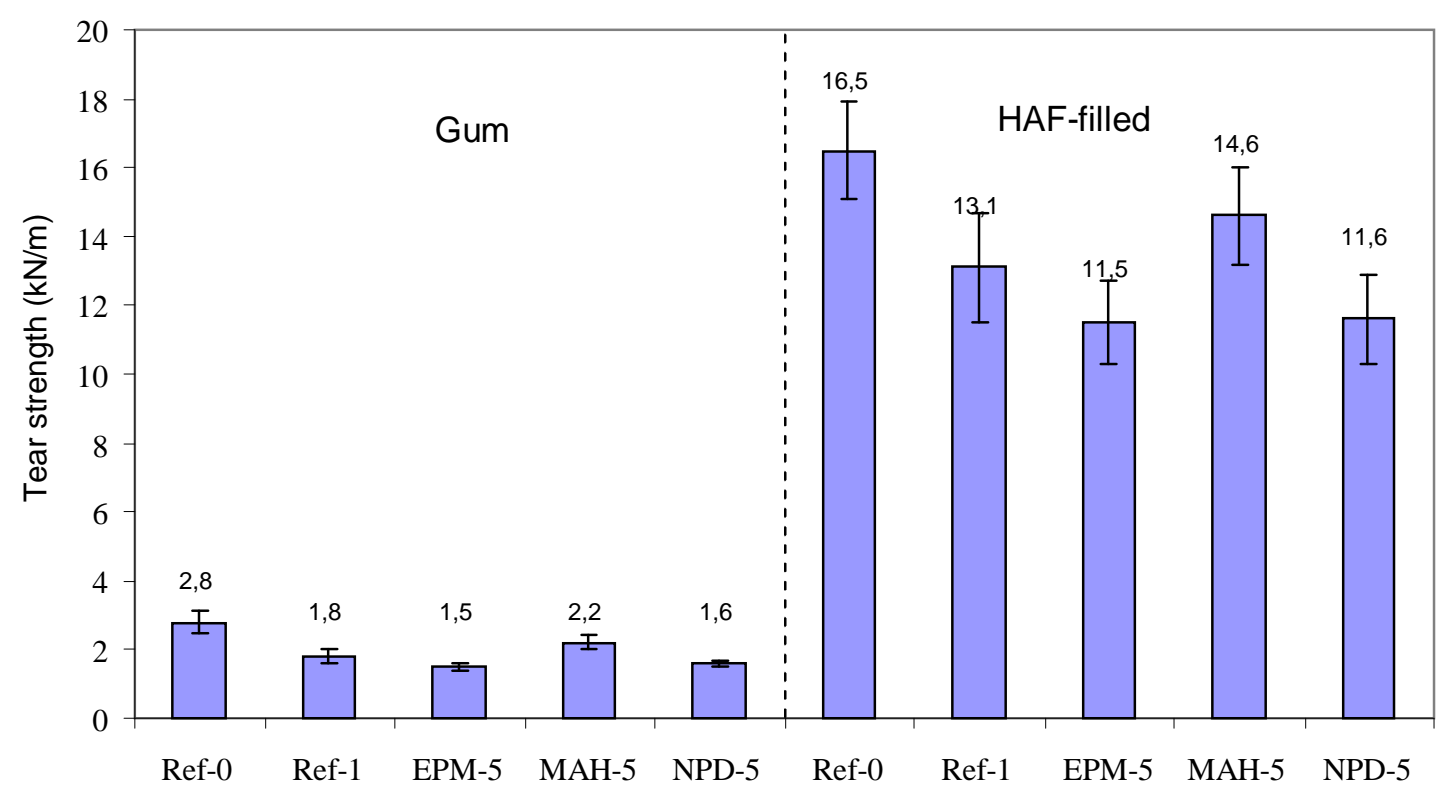

Figure 4.10 Trouser tear strength of the blends.

\subsubsection{Fatigue to failure properties}

Fatigue to failure is the tendency of a material to rupture by means of progressive cracks under stress-strain cycles. According to the ASTM D 4482 test standard, the fatigue data primarily give an estimation of the crack initiation and growth behavior of a rubber vulcanisate.

The results of fatigue-to-failure for the fixed extension ratio of 2 for both the gum blends and the HAF-filled blends are shown in figure 4.11. Ref-0 shows a superior fatigue life compared to the other blends. Among the EPDM-containing blends, the addition of EPM, MAH-EPM nor NPD-EPM increases the fatigue life at all in comparison with the straight NR/BR/EPDM blend. The fatigue life of HAF-filled $\mathrm{MAH}-5$ is even lower than HAF-filled Ref-1. For a fatigue life test based on the same extension, the modulus of the sample also plays an important role. The energy required for each flex cycle of a sample can be estimated by calculating the area under the stress-strain curve. It is clear that, for the same extension ratio more energy will be consumed during each cycle for higher modulus specimens and therefore, a shorter fatigue life may be obtained. In the present case, the lower fatigue life for all EPDM or EPDM/EPM blends can therefore well be due to their higher moduli as compared to the straight NR/BR compound. In order to exclude the influence from these different moduli, the test was repeated for the same strain energy for Ref-0, Ref- 1 and MAH-5. The results are shown in figure 4.12. 


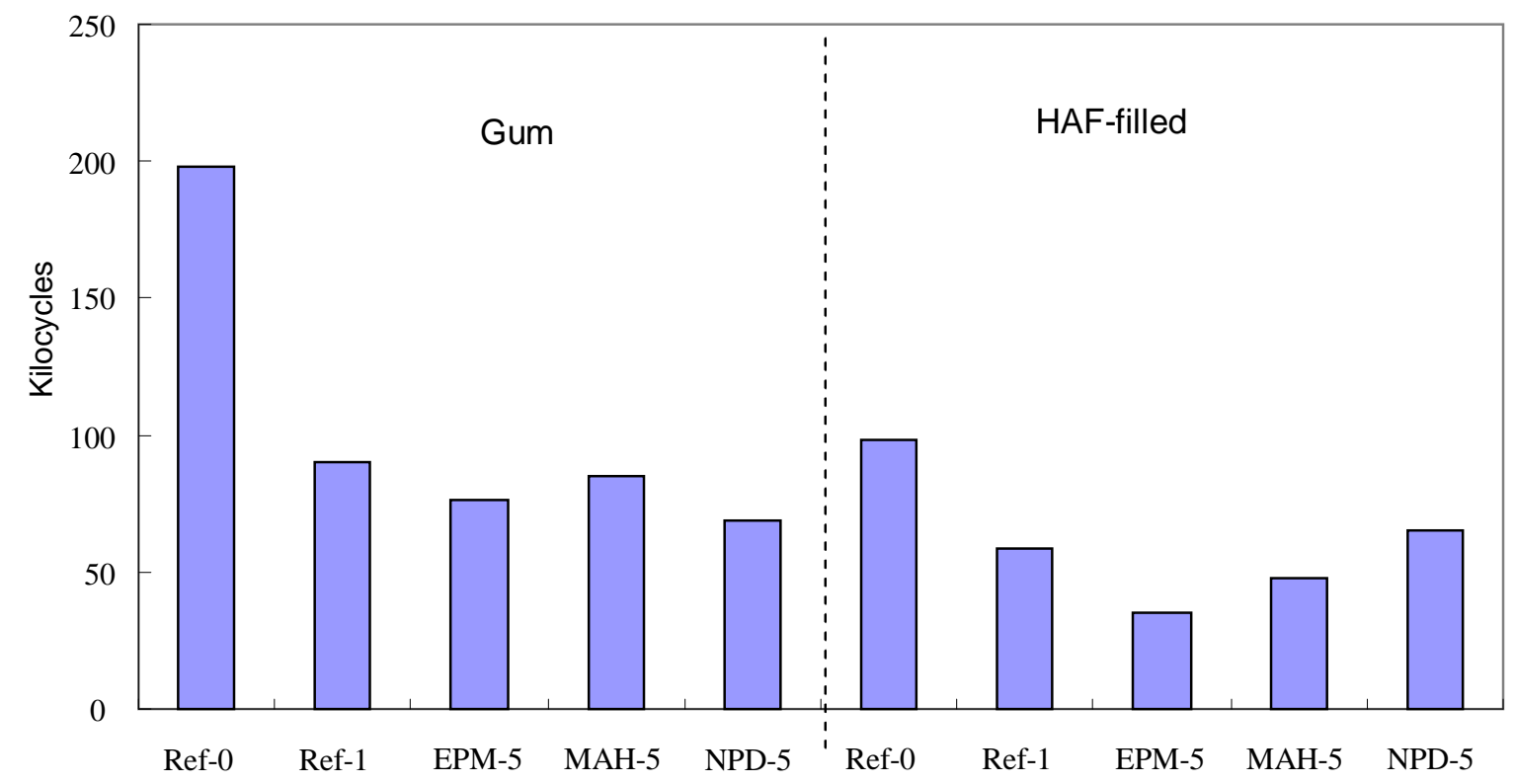

Figure 4.11 Fatigue-to-failure of the blends at an extension ratio of 2.

Considering now the fatigue life at constant strain energy of the gum blends, both Ref- 1 and MAH- 5 show a substantially longer fatigue life than Ref-0. In case of the HAF-filled blends, Ref-1 still has a shorter fatigue life than Ref-0. However, MAH5 shows a significantly longer fatigue life than Ref- 1 and Ref- 0 together. Therefore, the addition of MAH-EPM does lead to a better fatigue-to-failure performance than a straight NR/BR/EPDM blend for HAF-filled blends. The primary controlling parameters affecting the fatigue life are inhomogeneities or flaws present in a sample, caused by the blending, mixing, curing and sample cutting ${ }^{26}$. The longer fatigue life of HAF-filled-MAH-5 confirms a better blend homogeneity compared to the straight NR/BR/EPDM blend, especially the improvement of filler distribution among the various rubber phases in the blend. 


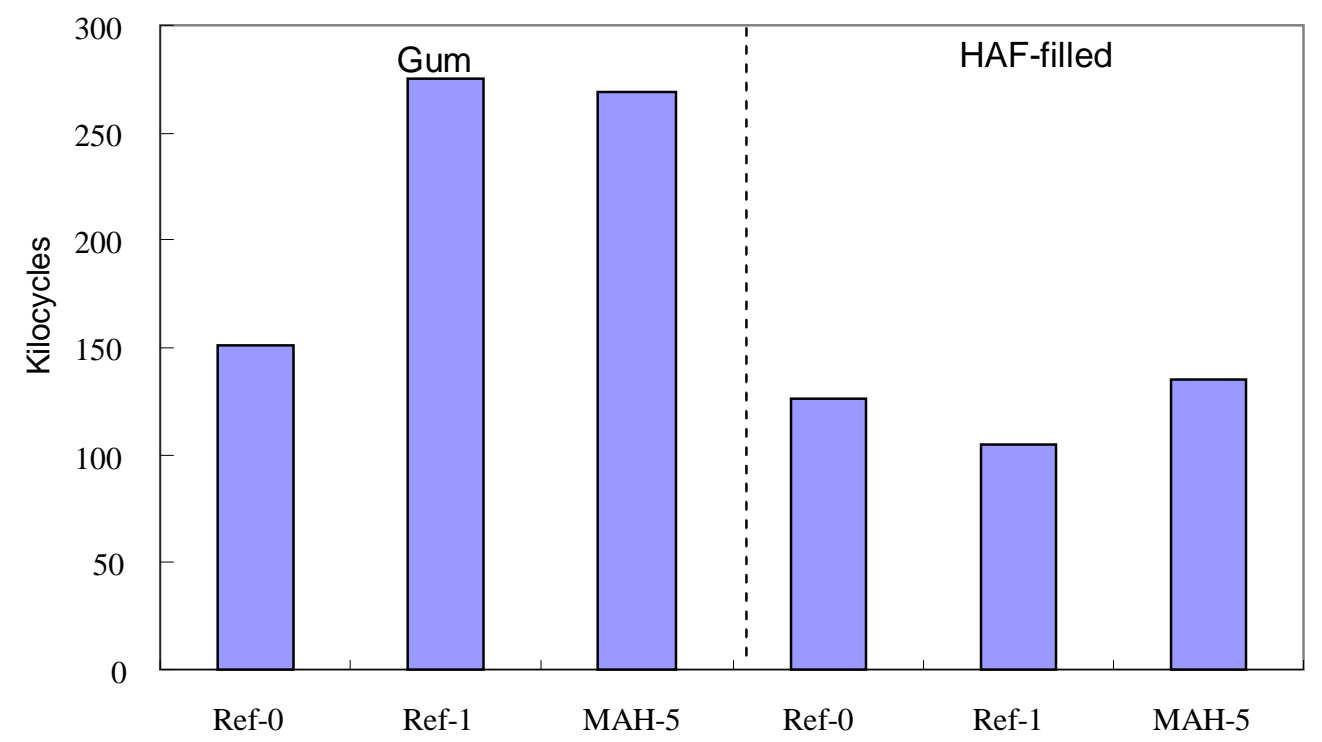

Figure 4.12 Fatigue-to-failure of the blends at a constant strain energy: (a) gum blends at 1.2MPa; (b)HAF-filled blends at 1.8MPa.

\subsubsection{Static and dynamic ozone resistance}

The static and dynamic ozone tests illustrate the resistance to ozone cracking of the NR/BR/EPDM/EPM blends in comparison with the conventional NR/BR blends with 6PPD as antiozonant. All specimens, except for HAF-filled Ref-0, are still free of cracks after the static ozone test for 192 hours. First cracks start showing on the surface of HAF-filled Ref-0 only after 120 hours, with the first 96 hours at 50ppm ozone concentration and afterwards at 100pphm (see experimental), as shown in figure 4.13 .

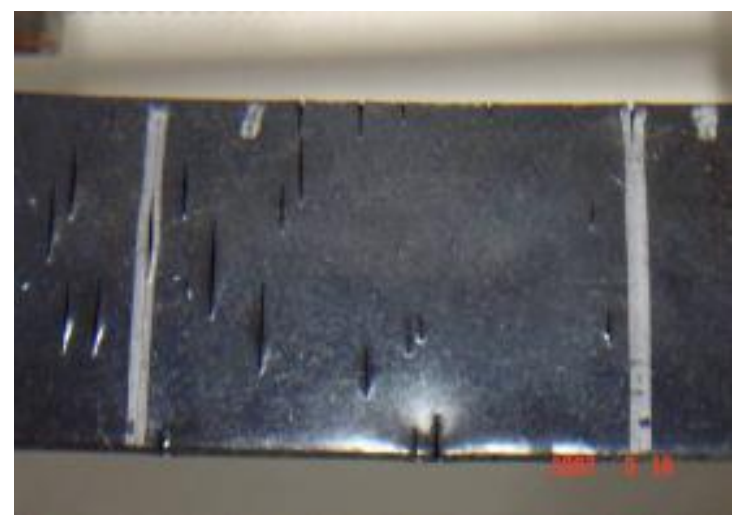

Figure 4.13 HAF-filled compound Ref-0 after the static ozone test.

A subsequent series of dynamic ozone tests were carried out on the same specimens with the test conditions described in Table 4.3. After the $1^{\text {st }}$ and $2^{\text {nd }}$ testrun, there were no cracks observed on the surface of any test specimens. However, 
as shown in figure 4.14, many small cracks can be observed on the edge of HAFfilled Ref- 0 blend. The gum and HAF-filled Ref- 0 blends also clearly show surface discoloration and staining, caused by the use of 6PPD in the compounds. The staining is more obvious in the dynamic test than in the static case, mainly because the stretch cycles of the specimens accelerate the migration of 6PPD to the surface.

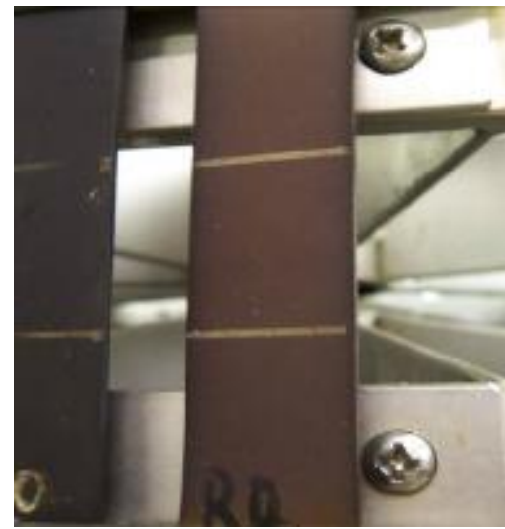

Gum-Ref-0

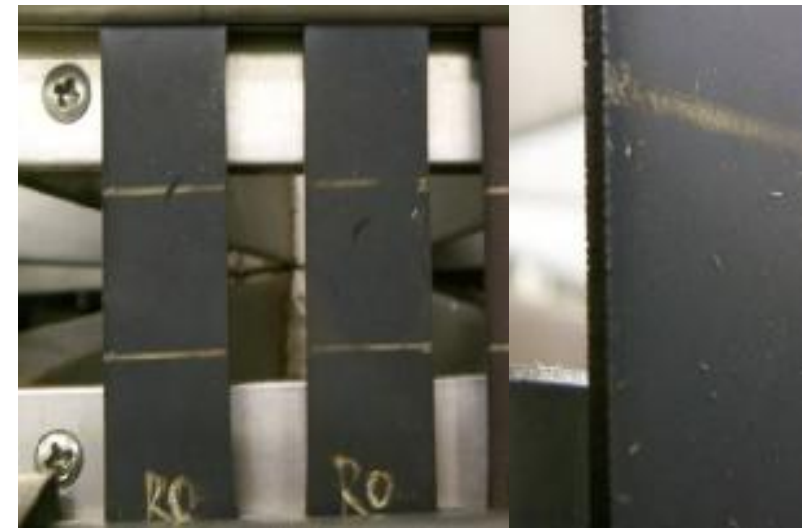

Filled-Ref-0

Figure 4.14 Gum and Filled Ref-0 blends after the $2^{\text {nd }}$ dynamic test-run.

After the $3^{\text {rd }}$ test-run at $30 \%$ elongation and 100 pphm ozone concentration, the gum-Ref-0 blend shows many small cracks on the edge. All the other gum blends containing EPDM also show cracks on the edge as well as on the surface of the elongated area, as shown in figure 4.15.

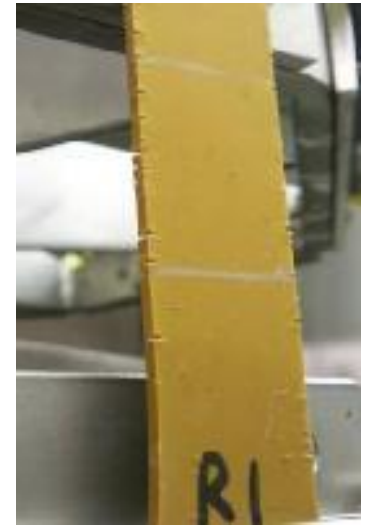

Gum-Ref-1

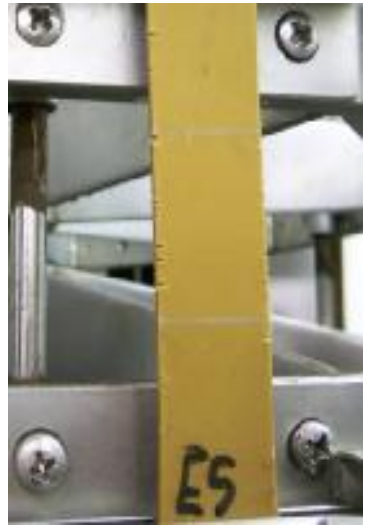

Gum-EPM-5

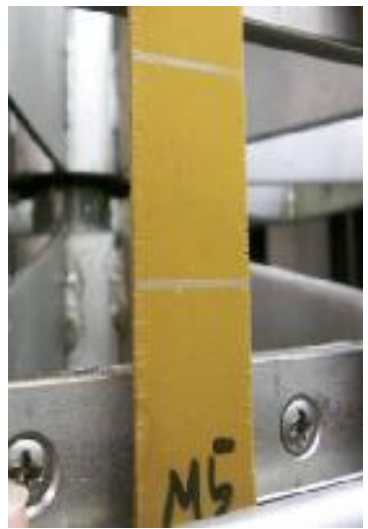

Gum-MAH-5

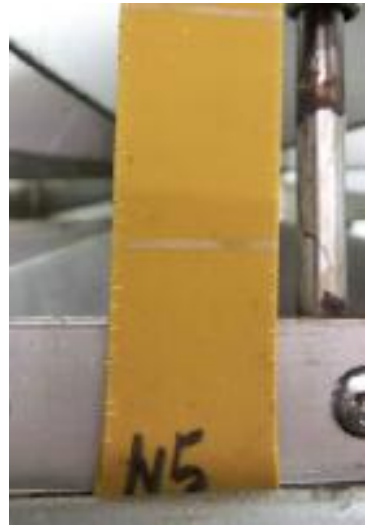

Gum-NPD-5

Figure 4.15 Gum Ref-1, EPM-5, MAH-5 and NPD-5 blends after the $3^{\text {rd }}$ dynamic test-run.

Cracks can also be observed on the HAF-filled blends as shown in figure 4.16. Besides many small cracks on the edge which were already seen after the dynamic second test-run, numerous tiny cracks on the surface of HAF-filled Ref-0 can be also observed under a microscope. The test specimens of filled Ref-1, EPM5, MAH-5 and NPD-5 show a lower number of cracks and better surface appearance. 
However, these specimens ruptured soon after the development of the cracks, due to lower cut growth resistance. Moreover, during the dynamic ozone test the specimens underwent stress-strain cycles similar to a fatigue to failure test at constant extension. The approximately $20 \%$ higher modulus of the EPDM containing blends, as given before implies a larger strain energy involved in the cycles and may to a large extent be held responsible for the earlier rupture.

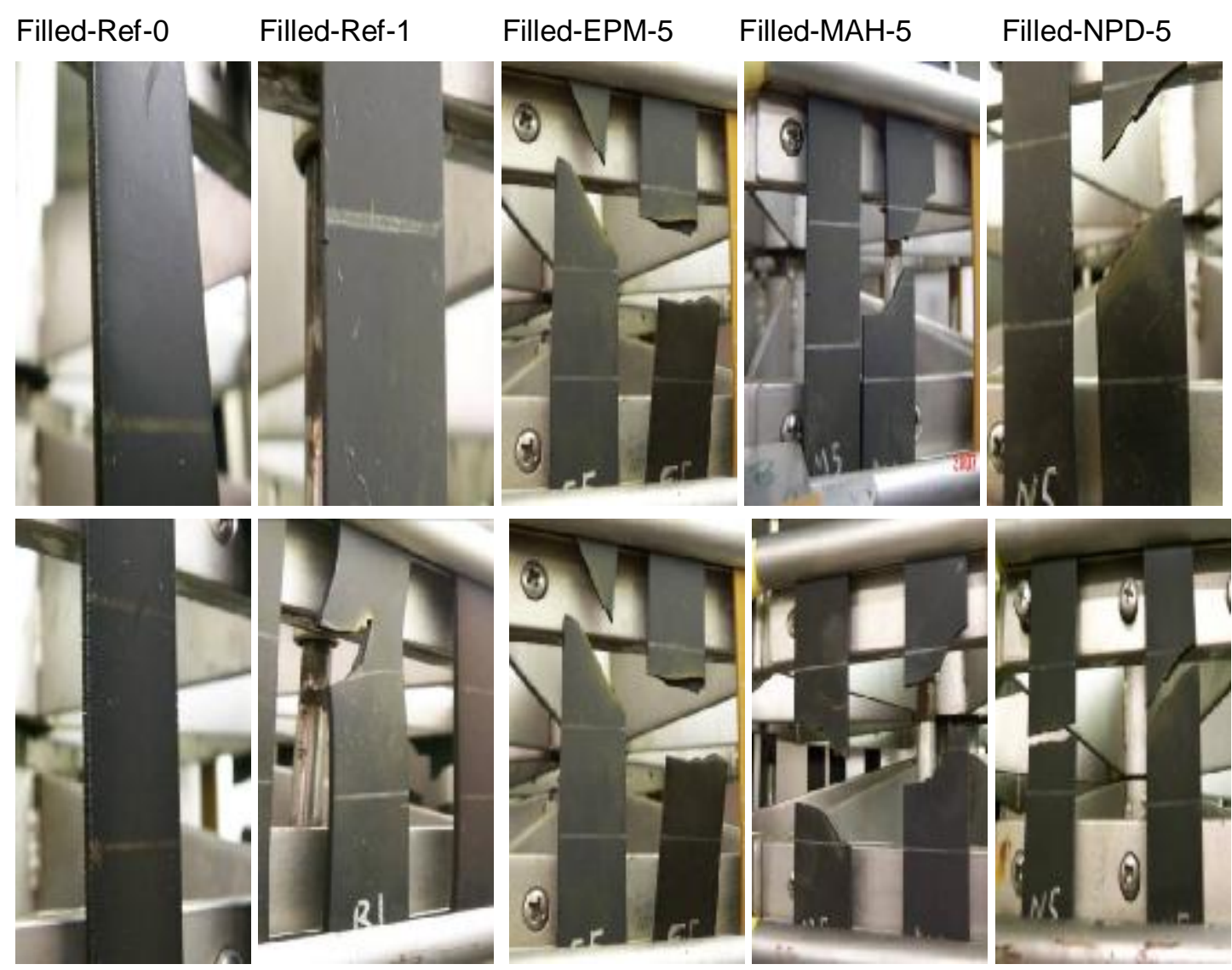

Figure 4.16 HAF-filled blends during the $3^{\text {rd }}$ dynamic test-run, after 24 hours (top) and after 48 hours (bottom).

From the results of the static and dynamic ozone tests it can be concluded, that the addition of $30 \mathrm{phr}$ of EPDM with or without EPMs into the conventional NR/BR blend gives the blends a comparable resistance to ozone cracking, and particularly better in static testing, as compared to 2 phr of 6PPD present in the recipe of Ref- 0 . It reconfirms that 6PPD can be omitted from the compound formulation by incorporation of EPDM. Meanwhile, the EPDM containg blends show excellent physical appearances because of the absence of the staining antiozonant. Considering the ozone resistance of the EPDM containing blends, all of them show a similar quality, suggesting that the resistance to ozone cracking is caused by the presence of a sufficient amount of highly saturated EPDM either with or without EPMs, irrespective of the crosslinking, filler distribution and blend morphology. 


\subsection{Conclusions}

MAH-EPM was added as compatibilising agent to NR/BR/EPDM blends, both gum and HAF-filled. Straight EPM an NPD-EPM were also studied for comparison. The mechanical properties of NR/BR/EPDM rubber blends are clearly improved by the addition of $5 \mathrm{phr}$ MAH-EPM. Straight EPM and NPD-EPM do not bring improvement to the blends properties. The tensile strength of the MAH-EPM compatibilised blend is much higher than the straight NR/BR/EPDM blend and is even very close to the NR/BR tyre sidewall compound. Higher modulus, better trouser tear strength and slightly increased elongation at break are also obtained by applying MAH-EPM to the NR/BR/EPDM blends. An improvement of the fatigue-tofailure property is also observed after the addition of MAH-EPM, when the test is carried out at constant strain energy instead of at the same extension. All these improvements suggest that MAH-EPM does improve the compatibility and the carbon black distribution between NR/BR and EPDM, next to some smaller effect of additional ionic crosslinking with $\mathrm{ZnO}$. The ozone resistance under both static and dynamic conditions of all the NR/BR/EPDM and NR/BR/EPDM/EPMs blends with 30phr of EPDM and EPMs is comparable to the NR/BR compounds with 6PPD. The anti-ozonant 6PPD can therefore be omitted from 30phr EPDM-containing tyre sidewall compounds.

\section{REFERENCES}

1. Avgeropoulos, G. N.; Weissert, F. C., Rubber Chem. Technol., 49, 93 (1976).

2. Bhowmick, A. K.; De, S. K., Rubber Chem. Technol., 53, 960 (1980).

3. Huntink, N. M.; Datta, R. N.; Noodermeer, J. W. M., Rubber Chem. Technol., 77, 476 (2003).

4. Corish, P. J.; Powell, B. D. W., Rubber Chem. Technol., 47, 481 (1974).

5. Roland, C. M., Rubber Chem. and Technol., 62, 456 (1989).

6. Chapman, A. V.; Tinker, A. J., Kautsch. Gummi Kunstst., 56, 533 (2003).

7. Ignatz-Hoover, F.; To, B. H.; Datta, R. N.; Hoog, A. J. d.; Huntink, N. M.; Talma, A. G., Rubber Chem. Technol., 76, 747 (2003).

8. Guillaumond, F. X., Rubber Chem. Technol., 105 (1976).

9. Ven, P.M. van de; Noordermeer, J. W. M., Rubber World, 222, 55 (2000).

10. Kluppel, M.; Schuster, R. H.; Schaper, J., Rubber Chem. Technol., 72, 91 (1999).

11. Callan, J. E.; Hess, W. M.; Scott, C. E., Rubber Chem. Technol., 44, 814 (1971).

12. Chang, Y.; Shin, Y., J. Appl. Polym. Sci., 73, 749 (1999).

13. Setua, D. K.; White, J. L., Polym. Eng. Sci., 31, 742 (1991).

14. Setua, D. K.; Pandey, K. N., J. Appl. Polym. Sci., 74, 480 (1999). 
15. Baranwal, K. C.; Son, P. N., Rubber Chem. Technol., 47, 88 (1974).

16. Ghosh, A. K.; Basu, D. K., Kautsch. Gummi Kunstst., 56, 101 (2003).

17. Oliveira, M. G.; Soares, B. G., Macromol. Rapid Commun., 20, 526 (1999).

18. Hopper, R. J., Rubber Chem. Technol., 49, 341 (1976).

19. Cook, S., in "Blends of Natural Rubber", Chapman \& Hall, 1998.

20. Sahakaro, K.; Talma, A. G.; Datta, R. N.; Noordermeer, J. W. M., J. Appl. Polym. Sci., 103, 2547 (2007).

21. Coran, A. Y., Rubber Chem. Technol., 61, 281 (1988).

22. Layer, R. W.; Lattimer, R. P., Rubber Chem. Technol., 63, 426 (1990).

23. Waddell, W. H., Rubber Chem. Technol., 71, 590 (1998).

24. Sumner, A. J. M.; Fries, H., Kautsch. Gummi Kunstst., 45, 558 (1992).

25. Andrews, E. H., Rubber Chem. Technol., 40, 635 (1967).

26. Young, D. G.; Kresge, E. N.; Wallace, A. J., Rubber Chem. Technol., 55, 428 (1982). 


\section{Chapter 5}

\section{Maleic-Anhydride Grafted EPM as Compatibilising Agent in NR/BR/EPDM Blends: Part II. Mechanistic Aspects}

Poor mechanical properties of polydiene rubber/EPDM blends are commonly due to cure and phase incompatibility, and to a heterogeneous filler distribution in each of the rubber phases. In the previous study of this series, it was demonstrated that the mechanical properties of NR/BR/EPDM blends can be significantly improved by addition of $5 \mathrm{phr}$ maleic-anhydride grafted EPM (MAH-EPM). In the present paper, it is shown that the improvement can mainly be attributed to a compatibilising effect of MAH-EPM, resulting in a more homogeneous phase distribution, but in particular a much better homogeneous carbon black distribution over the different rubber phases. In addition, ionic crosslinks are introduced into the blends by interaction of MAH-EPM with zinc oxide. 


\subsection{Introduction}

In the first part of this series: Chapter 4, three types of EPMs were investigated for NR/BR/EPDM blends for their ability for enhance compatibility and to bridge the polarity difference. Neither straight EPM nor N-phenyl-p-phenylenediamine modified EPM (NPD-EPM) enhances the mechanical properties of such blends. However, the mechanical properties are significantly improved by the addition of $5 \mathrm{phr}$ maleicanhydride grafted EPM (MAH-EPM), resulting in increased tensile strength, higher modulus, better trouser tear strength, and increased fatigue life based on constant strain energy.

Nowadays, MAH-modified polyolefins, such as polyethylene (PE), polypropylene (PP), ethylene propylene copolymers [EP(D)M], hydrogenated styrene-butadiene-styrene block copolymers (SEBS), are produced on a commercial scale and have found widespread application as reactive compatibilisers or adhesives for apolar polyolefins to polar materials, as a result of their increased polarity and reactivity ${ }^{1-4}$. In addition, MAH-modified polyolefins can be further modified with for instance amines and alcohols, yielding imides and esters, respectively.

Grafting of $\operatorname{EP}(D) M$ with maleic anhydride yields an elastomer, which is characterised by local polarity and chemical reactivity. Coran ${ }^{5,6}$ found that if the EPDM molecules are functionalised by maleic-anhydride or maleic acid, the mechanical properties of vulcanised blends of the functionalised EPDM with NR are greatly improved. One of the possible reasons is that the EPDM can be independently crosslinked by the action of the zinc oxide present in the acceleratedsulphur vulcanising system. Thus an ionic crosslink network can be formed in the EPDM microdomains, which is not competitive with the accelerated-sulphur vulcanisation system which reacts rapidly with the NR. MAH-modified EPM resembles $\mathrm{MAH}$-modified EPDM in structure, only without the double bond. The polarity, chemical reactivity and ionic crosslinking due to the maleic anhydride group will still play an important role in polydiene rubber/EPDM/MAH-EPM blends.

In the present paper, the objective is to study the mechanism involved in a MAH-EPM compatibilised blend of NR, BR and EPDM, and to create a better understanding of the significant improvements in mechanical properties compared to a straight NR/BR/EPDM blend. Several possible reasons may explain these improvements by the MAH-EPM:

1) Increased solubility of curatives in the EPDM phase due to a polarity change;

2) Compatibilising effect between NR/BR and EPDM rubbers;

3) Better filler distribution; 
4) Additional ionic crosslinks by the presence of MAH-EPM.

\subsection{Experimental}

\subsubsection{Materials}

The blends consist of the same ingredients as mentioned in part I of this series: Chapter 4.

The staining agent for the TEM experiment was a $2 \%$ osmic acid solution in water (Merck).

The vulcanising agent for the solubility study is $40 \%$ pure Dicumyl peroxide (Perkadox ${ }^{\circledR}$ BC-40B, Akzo Nobel, the Netherlands).

\subsubsection{Preparation of blend compounds}

The overall recipes for both gum and filled blends are given in Table 5.I. The preparation of the various compounds was described in detail in part-I of this series: Chapter 4.

Table 5.1 Formulations of NR/BR/EPDM/EPM compounds: gum stocks and black reinforced (phr).

\begin{tabular}{|c|c|c|c|c|c|c|c|c|c|c|}
\hline \multirow{2}{*}{ Component } & \multicolumn{5}{|c|}{ Gum } & \multicolumn{5}{|c|}{ HAF-filled } \\
\hline & Ref-0 & Ref-1 & EPM-5 & MAH-5 & NPD-5 & Ref-0 & Ref-1 & EPM-5 & MAH-5 & NPD-5 \\
\hline NR & 50 & 35 & 35 & 35 & 35 & 50 & 35 & 35 & 35 & 35 \\
\hline BR & 50 & 35 & 35 & 35 & 35 & 50 & 35 & 35 & 35 & 35 \\
\hline EPDM & 0 & 30 & 25 & 25 & 25 & 0 & 0 & 25 & 25 & 25 \\
\hline EPM & 0 & 0 & 5 & 0 & 0 & 0 & 0 & 5 & 0 & 0 \\
\hline MAH-EPM & 0 & 0 & 0 & 5 & 0 & 0 & 0 & 0 & 5 & 0 \\
\hline NPD-EPM & 0 & 0 & 0 & 0 & 5 & 0 & 0 & 0 & 0 & 5 \\
\hline HAF N330 & 0 & 0 & 0 & 0 & 0 & 50 & 50 & 50 & 50 & 50 \\
\hline $\begin{array}{c}\text { Sunthene } \\
4240\end{array}$ & 0 & 0 & 0 & 0 & 0 & 10 & 10 & 10 & 10 & 10 \\
\hline $\mathrm{ZnO}$ & 4 & 4 & 4 & 4 & 4 & 4 & 4 & 4 & 4 & 4 \\
\hline $\begin{array}{c}\text { Stearic } \\
\text { acid }\end{array}$ & 2 & 2 & 2 & 2 & 2 & 2 & 2 & 2 & 2 & 2 \\
\hline $\mathrm{TMQ}$ & 1 & 1 & 1 & 1 & 1 & 1 & 1 & 1 & 1 & 1 \\
\hline 6PPD & 2 & 0 & 0 & 0 & 0 & 2 & 0 & 0 & 0 & 0 \\
\hline CBS & 2 & 2 & 2 & 2 & 2 & 2 & 2 & 2 & 2 & 2 \\
\hline Sulphur & 2.5 & 2.5 & 2.5 & 2.5 & 2.5 & 2.5 & 2.5 & 2.5 & 2.5 & 2.5 \\
\hline $\begin{array}{c}\text { Total } \\
\text { weight }\end{array}$ & 113.5 & 111.5 & 111.5 & 111.5 & 111.5 & 173.5 & 171.5 & 171.5 & 171.5 & 171.5 \\
\hline
\end{tabular}




\subsubsection{Vulcanisation}

The optimum cure time $t_{c, 90}$ of the fully compounded blends was determined as the time needed to reach $90 \%$ of the maximum torque in a Rubber Process Analyzer RPA2000 of Alpha Technologies at $140^{\circ} \mathrm{C}, 0.833 \mathrm{~Hz}$ and 0.2 degree strain. The blends were then vulcanised for the optimum cure time $t_{c, 90}$ in a Wickert WLP1600 laboratory compression press at $140^{\circ} \mathrm{C}$ and 100 bar into $2 \mathrm{~mm}$ thick sheets.

\subsubsection{Characterisation}

\subsubsection{Solubility of curatives}

The solubilities of sulphur and CBS in pure EPDM and in the EPDM/MAHEPM (25/5) blend (without NR and BR included) were tested according to the method of Morris and Thoma ${ }^{7}$. To avoid crystallisation of the curatives within the rubber samples, they were first vulcanised with $2.5 \mathrm{phr}$ dicumyl peroxide (40\% purity) for 15 minutes at $160^{\circ} \mathrm{C}$ before the measurement. Then the vulcanised samples were cut into sheets with size: $1 \mathrm{~cm}^{*} 1 \mathrm{~cm}^{\star} 2 \mathrm{~mm}$. Samples were extracted with hot acetone in a soxhlet extractor for 48 hours to remove the non-rubber parts and dried in a vacuum oven. The samples were then placed in a glass bottle and packed on all sides with sulphur or CBS in the form of fine powder. The measurement was carried out at $60^{\circ} \mathrm{C}$. After certain time intervals, the samples were taken out and their surfaces cleaned first by a sharp knife and then by adhesive tape before weighing. This process was repeated till no further weight increase over time did occur.

\subsubsection{Transmission electron microscopy (TEM)}

$70 \mathrm{~nm}$ thin sections of the blend vulcanisates were cryogenically cut with a diamond knife using a Leica ultra microtome at $-150^{\circ} \mathrm{C}$. The sections were deposited on a carbon support film on copper grid (200 mesh) and then vapor stained using $2 \%$ osmium tetroxide for 15 minutes. TEM measurements were performed with a Philips CM 30 transmission electron microscope.

\subsubsection{Compression set}

Compression set was determined according to ASTM D395 after 22hrs exposure to three different temperatures: $23^{\circ} \mathrm{C}, 70^{\circ} \mathrm{C}$ and $100^{\circ} \mathrm{C}$.

\subsubsection{Strain sweep measurements}

Storage modulus (G') measurements were done using the Rubber Process Analyzer RPA 2000 of Alpha Technologies at a frequency of $0.5 \mathrm{~Hz}$ and at different 
temperatures: $100^{\circ} \mathrm{C}, 110^{\circ} \mathrm{C}$ and $120^{\circ} \mathrm{C}$ for uncured compounds. A strain of $100 \%$ was applied.

\subsection{Results and discussion}

\subsubsection{Solubility of curatives in EPDM and EPDM/MAH-EPM rubbers}

The difference in the solubility of curatives in different elastomers is one of the most important aspects which contributes to the cure incompatibility in blends of dissimilar rubbers. It is well known that curatives, which are considered as polar species, generally diffuse more readily into higher polar rubber phases, resulting in differences in concentration between reactants and hence uneven crosslink distribution ${ }^{8-10}$. Increasing the solubility of curatives in the EPDM phase relative to the polydiene matrix is one of the possible approaches to solve the cure incompatibility problem. The question is whether the addition of MAH-EPM to pure EPDM does increase the solubility of curatives.

The weight increase of a rubber sheet after adsorption of a curative to its saturation level was measured at $60^{\circ} \mathrm{C}$. The values of weight increase of sulphur and CBS in pure EPDM rubber and in the 25/5 EPDM/MAH-EPM blend are shown in Table 5.2. There is no significant difference between the solubilities of sulphur in pure EPDM rubber and in the EPDM/MAH-EPM blend, nor for CBS. It means that, although the addition of MAH-EPM may increase the polarity of EPDM, it is not significant enough to improve the solubility of curatives due to the limited and diluted polarity increase by only $2 \mathrm{wt} \%$ of maleic anhydride in the MAH-EPM. Consequently, this possible reason for the improvements obtained with the addition of MAH-EPM can be neglected.

Table 5.2 Solubility of sulphur and CBS in EPDM and EPDM/MAH-EPM compounds.

\begin{tabular}{c|cc}
\hline Compounds & Weight increase of S & Weight increase of CBS \\
\hline EPDM & $1.34 \%$ & $5.39 \%$ \\
EPDM/MAH-EPM & $1.31 \%$ & $5.05 \%$ \\
\hline
\end{tabular}

\subsubsection{Morphological mechanisms}

TEM was applied to characterise the phase morphology between the various rubbers due to an eventual compatibilising effect of the MAH-EPM, and its potential effect on filler distribution. The TEM-images of the gum blends are shown in figure 5.1. Due to the presence of three types of rubbers in the blends, the images are 
complicated. In the TEM experiments, the polydiene phases NR and BR, which are more stained by osmium tetroxide than EPDM, become dense areas with higher atomic number and appear dark in the images ${ }^{11}$. The EPDM phase contains a very small amount of double bonds in the molecule, and is by far less stained by osmium tetroxide and therefore appears light in the TEM images. The individual NR and BR components cannot be discriminated. It can be observed that the EPDM is dispersed in NR/BR matrix as particles with different sizes. Comparing the images of Ref- 1 and $\mathrm{MAH}-5$, the contrast between the different phases of Ref-1 is higher than that of $\mathrm{MAH}-5$. The EPDM particles of the MAH-5 blend are slightly smaller in average and finer dispersed in the NR/BR matrix. Even though the differences are small, a slightly more homogeneous morphology for the rubber blend after the addition of MAH-EPM indicates a compatibilising effect and/or a viscosity adjustment of the EPDM-phase.

Figure 5.2 shows the TEM-images of the HAF carbon black-filled blends of Ref- 1 and MAH-5. The carbon black-filled areas appear dark in the image. The light areas of the EPDM particles are not filled with carbon black. The image of MAH-5 clearly shows a more homogeneous morphology compared to Ref- 1 . It is not likely that this is the result of a better morphology between the various rubbers, because of the small effects seen in figure 5.1. It is most probably is the result of a better, more homogeneous carbon black dispersion. Normally the NR/BR phase has a higher affinity for carbon black than the EPDM phase. The addition of MAH-EPM apparently raises the polarity of the EPDM and therefore enhances the affinity of carbon black for the EPDM phase relative to the NR/BR phase.

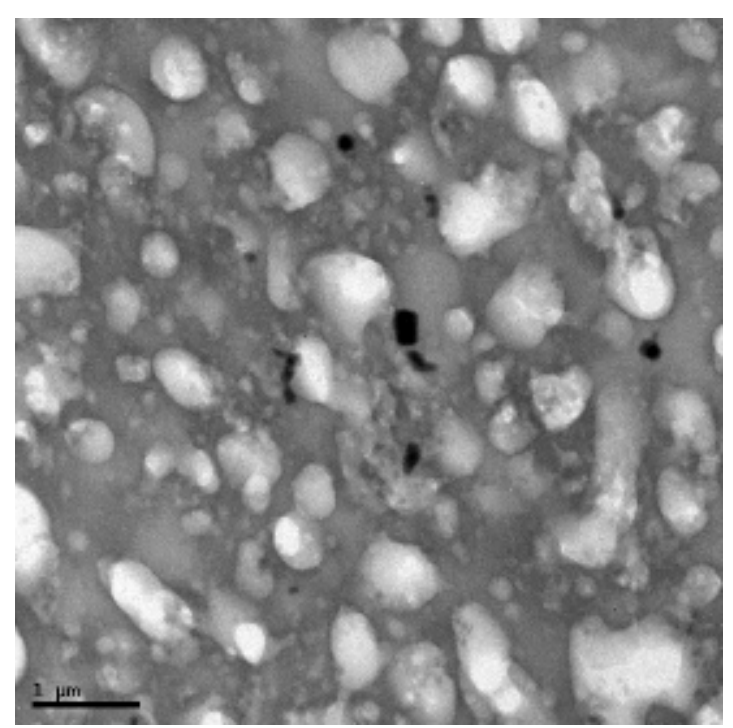

(a) Ref-1

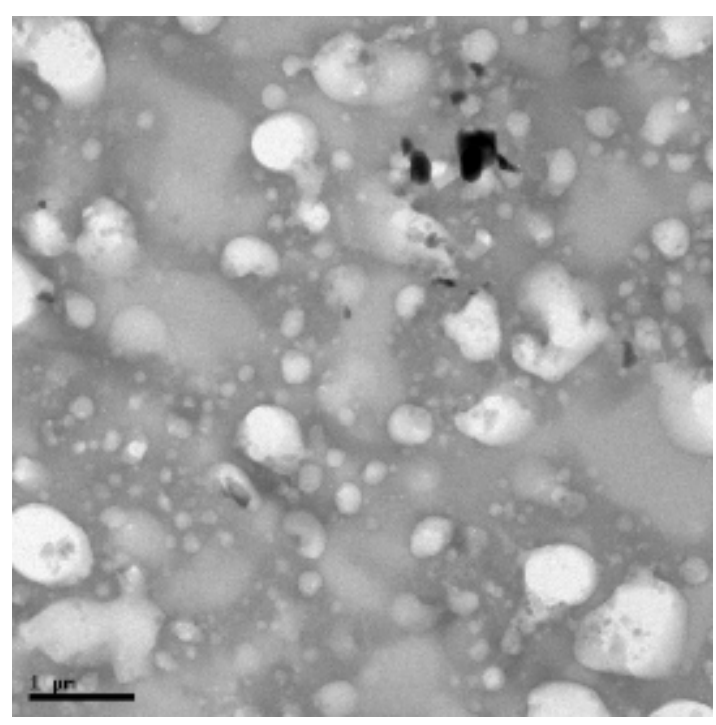

(b) $\mathrm{MAH}-5$

Figure 5.1 TEM images of gum blends at a magnification of 3200X. 


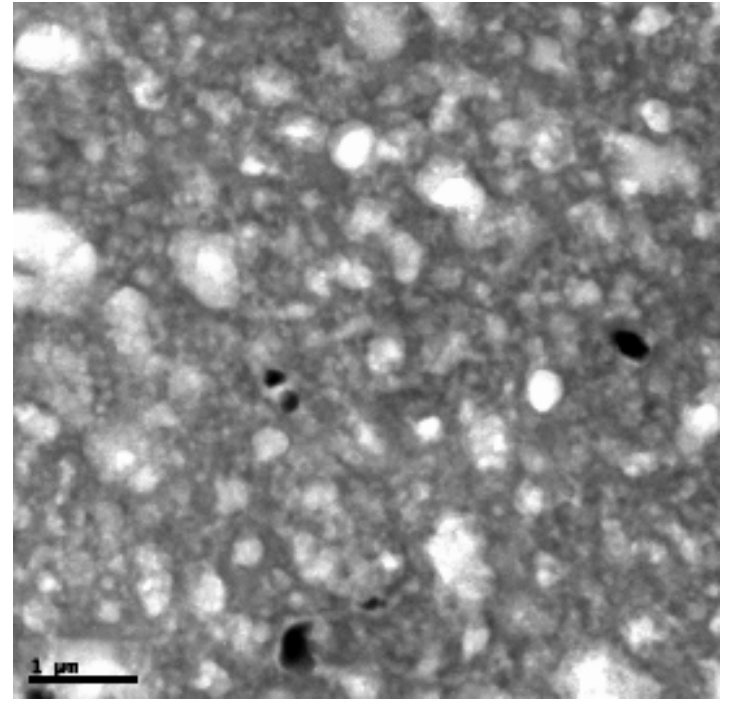

(a) Ref-1

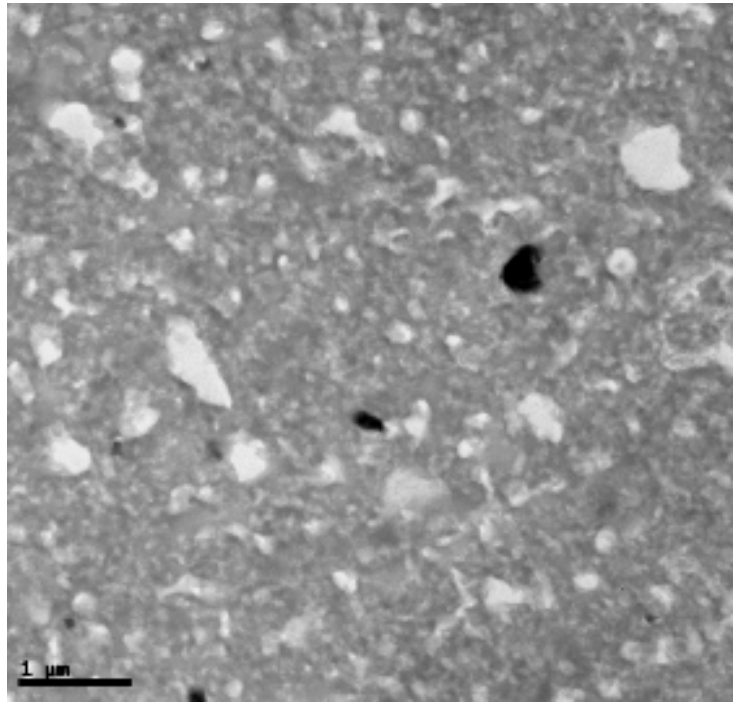

(b) MAH-5

Figure 5.2 TEM images of HAF-filled blends at a magnification of 3200X.

\subsubsection{Additional crosslinking by ionic coupling}

\subsubsection{Compression set}

The compression set data of gum and HAF-filled blends at different temperatures of $23^{\circ} \mathrm{C}, 70^{\circ} \mathrm{C}$ and $100^{\circ} \mathrm{C}$ are shown in figure 5.3. In both cases, the $\mathrm{NR} / \mathrm{BR}$ blends show the lowest compression set values compared to the EPDM containing blends, indicating a good elastic recovery property. At $23^{\circ} \mathrm{C}$, the differences of the compression sets between the EPDM containing blends are small for both gum and HAF-filled cases. However, MAH-5 still does show a slightly lower compression set compared to the other EPDM containing blends. When the temperature is increased to $70^{\circ} \mathrm{C}$, the lower compression set value or better elastic recovery property of MAH-5 is more significant compared to the other EPDM containing blends. The improvement of the compression set property at $23^{\circ} \mathrm{C}$ and $70^{\circ} \mathrm{C}$ after the addition of MAH-EPM suggests a higher crosslink density. 


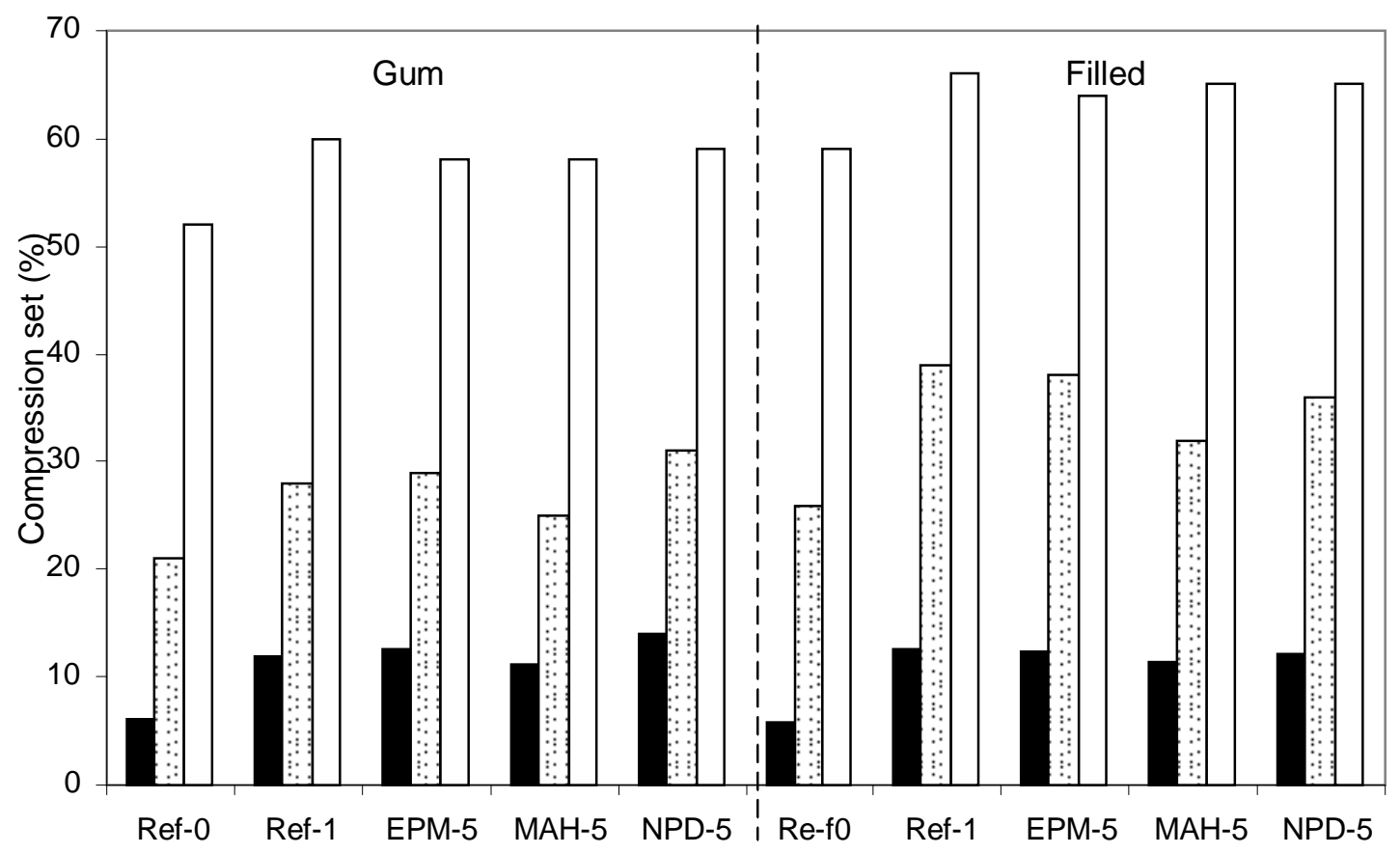

Figure 5.3 Compression set of the blends: (匹): $23^{\circ} \mathrm{C}$; (

At $100^{\circ} \mathrm{C}$, the difference in compression set between $\mathrm{MAH}-5$ and the other EPDM containing blends disappears, which means that the crosslink density in the $\mathrm{MAH}-5$ blends is the same compared to the other EPDM containing blends. Therefore, the higher crosslink density of the MAH-5 blends at lower temperatures must be due to some additional crosslinks, which are not stable and will decompose at elevated temperature. It has been observed by others that maleic anhydridegrafted EPM can be thermoreversibly crosslinked by ionic interaction with zinc (Zn) or potassium (K) cations as shown in Scheme $5.1^{12,13}$. Therefore it is logical to expect that additional ionic crosslinks are formed in the MAH-EPM containing blends at the lower temperature region, because of the presence of zinc oxide in the acceleratedsulphur vulcanising blends. 


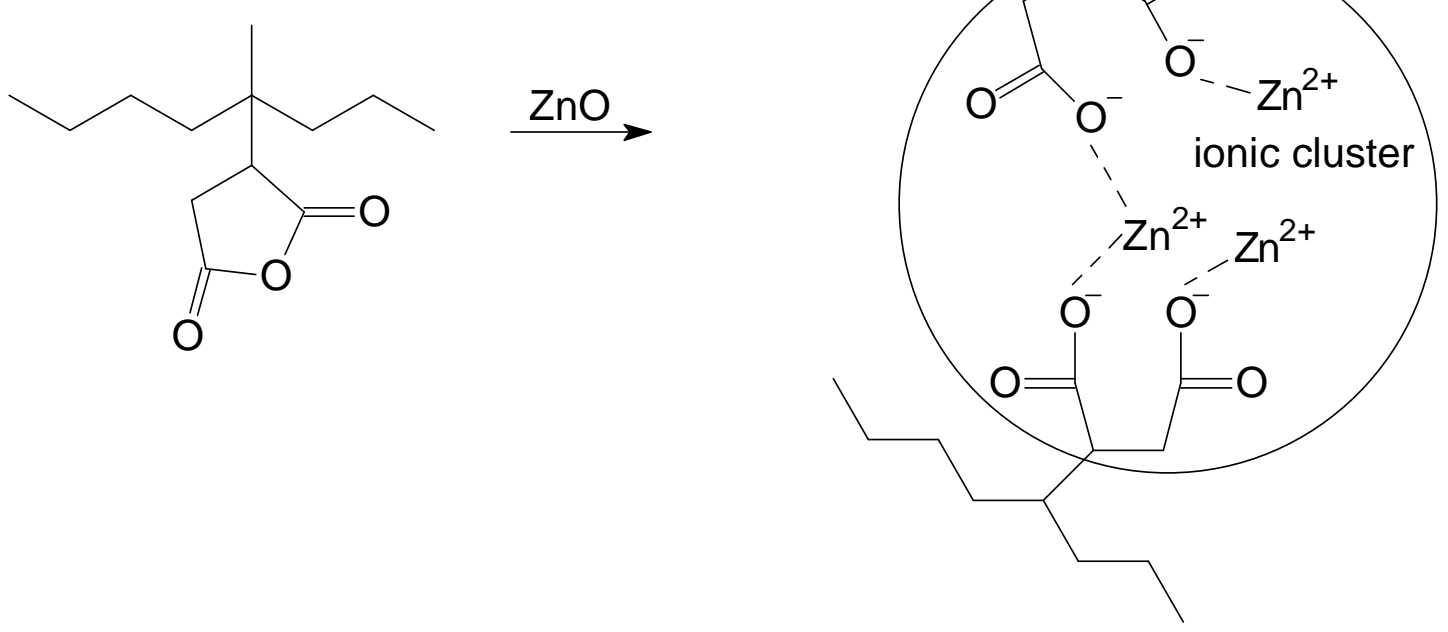

Scheme 5.1 Schematic representation of ionomers prepared by neutralisation of MAH-EPM with a zinc salt.

\subsubsection{Indications of ionic crosslinking from strain sweep measurements}

In a strain sweep measurement of a filler-reinforced rubber, the storage modulus G' at high strain is attributed to a combination of filler-polymer interactions, the hydrodynamic effect of the filler and the crosslinked polymer network ${ }^{14,15}$. The influence of the polymer crosslink-network on G' at high strain: $100 \%$, is of particular interest in this study. Figure 5.4 shows the effect of $\mathrm{ZnO}$ on G' at $100 \%$ strain for the unvulcanised HAF-filled NR/BR/EPDM/EPM blends at $100^{\circ} \mathrm{C}$. In the recipes as shown in Table 5.1, there are 4 phr of $\mathrm{ZnO}$ present in each compound. $\mathrm{MAH}-5$ shows an increased G' compared to the other blends in the presence of $\mathrm{ZnO}$. However, when $\mathrm{ZnO}$ is excluded from the formulations, the $\mathrm{G}^{\prime}$ value of $\mathrm{MAH}-5$ is at the same level as for the other blends. It confirms that additional crosslinks are formed in the $\mathrm{MAH}-\mathrm{EPM}$ containing blend in the presence of $\mathrm{ZnO}$. 


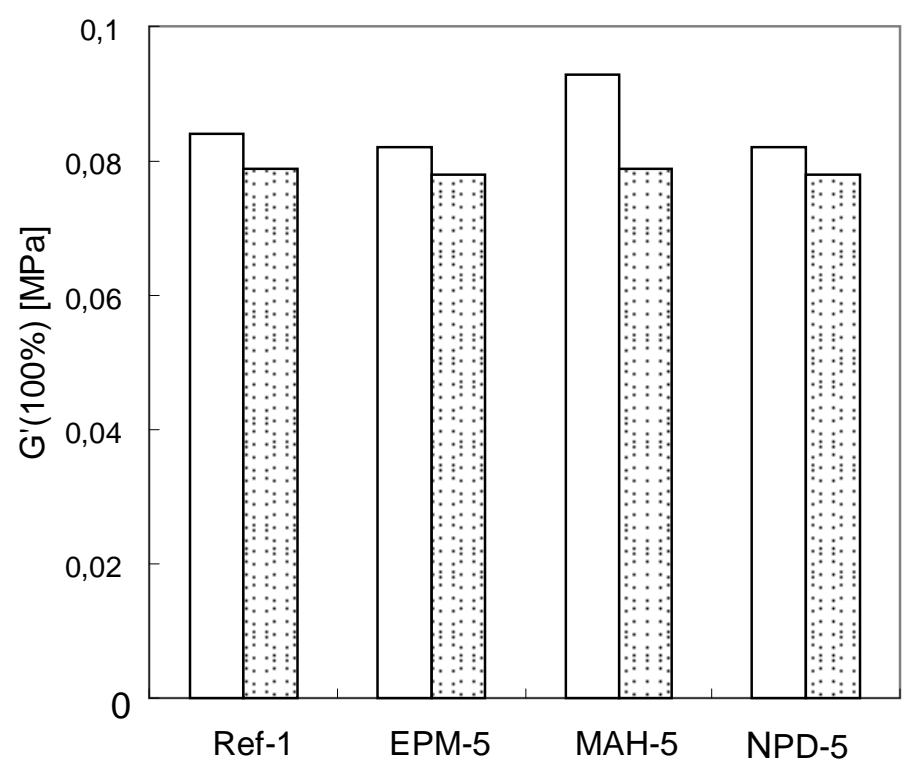

Figure 5.4 Effect of $\mathrm{ZnO}$ on the G' at $100 \%$ strain for NR/BR/EPDM/EPMs blends at $100^{\circ} \mathrm{C}$ : (): with $\mathrm{ZnO}$; ( (:iw): without $\mathrm{ZnO}$.

Above results show that the ionic crosslinks are still at least partially present at $100^{\circ} \mathrm{C}$, most probably due to a much shorter test time in strain sweep measurements: 10 minutes as compared to the compression set test: 22 hours. Figure 5.5 shows the influence of temperature and the amount of $\mathrm{ZnO}$ on $\mathrm{G}^{\prime}$ at $100 \%$ strain for the $\mathrm{NR} / \mathrm{BR} / \mathrm{EPDM} / \mathrm{MAH}$-EPM blend. At $100^{\circ} \mathrm{C}$, the addition of $1 \mathrm{phr} \mathrm{ZnO}$ already results a significant increase of $\mathrm{G}^{\prime}(100 \%)$. With more $\mathrm{ZnO}$, the increase is less pronounced. At higher temperatures of $110^{\circ} \mathrm{C}$ and $120^{\circ} \mathrm{C}$, all the compounds show comparable $\mathrm{G}^{\prime}(100 \%)$ values. At $120^{\circ} \mathrm{C}$ all the differences are gone. It proves that temperatures above $100^{\circ} \mathrm{C}$ accelerate the decomposition of the ionic crosslinks.

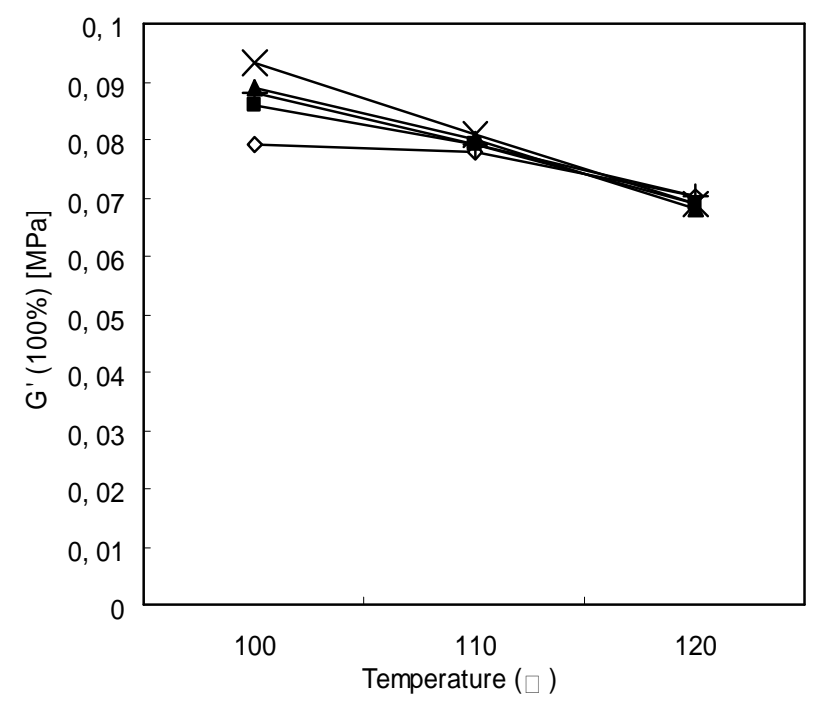

Figure 5.5 Effect of temperature and $\mathrm{ZnO}$-amount on $\mathrm{G}^{\prime}$ at $100 \%$ strain for $\mathrm{MAH}-5$ :

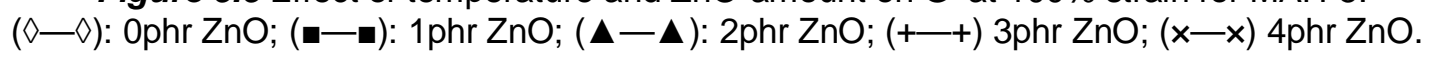


One sample of the NR/BR/EPDM/MAH-EPM blends without $\mathrm{ZnO}$ and another one with $4 \mathrm{phr} \mathrm{ZnO}$ were first measured at $120^{\circ} \mathrm{C}$. Measurement was repeated at $100^{\circ} \mathrm{C}$ after storage of the samples for 24 hours at room temperature (RT). Figure 5.6 shows the results of $\mathrm{G}^{\prime}(100 \%)$ values. At the initial $120^{\circ} \mathrm{C}$, the two blends have similar values because the ionic crosslinks in the blend with $\mathrm{ZnO}$ are decomposed. However, after 24hours storage at RT, the blend with $\mathrm{ZnO}$ shows a much higher $\mathrm{G}^{\prime}(100 \%)$ value again than the one without $\mathrm{ZnO}$ at $100^{\circ} \mathrm{C}$, indicating that the additional crosslinks are present again. The additional ionic crosslinks formed in MAH-EPM with Zn-cations are thermoreversible, which corresponds to the wellknown nature of ionic interaction ${ }^{16,17}$.

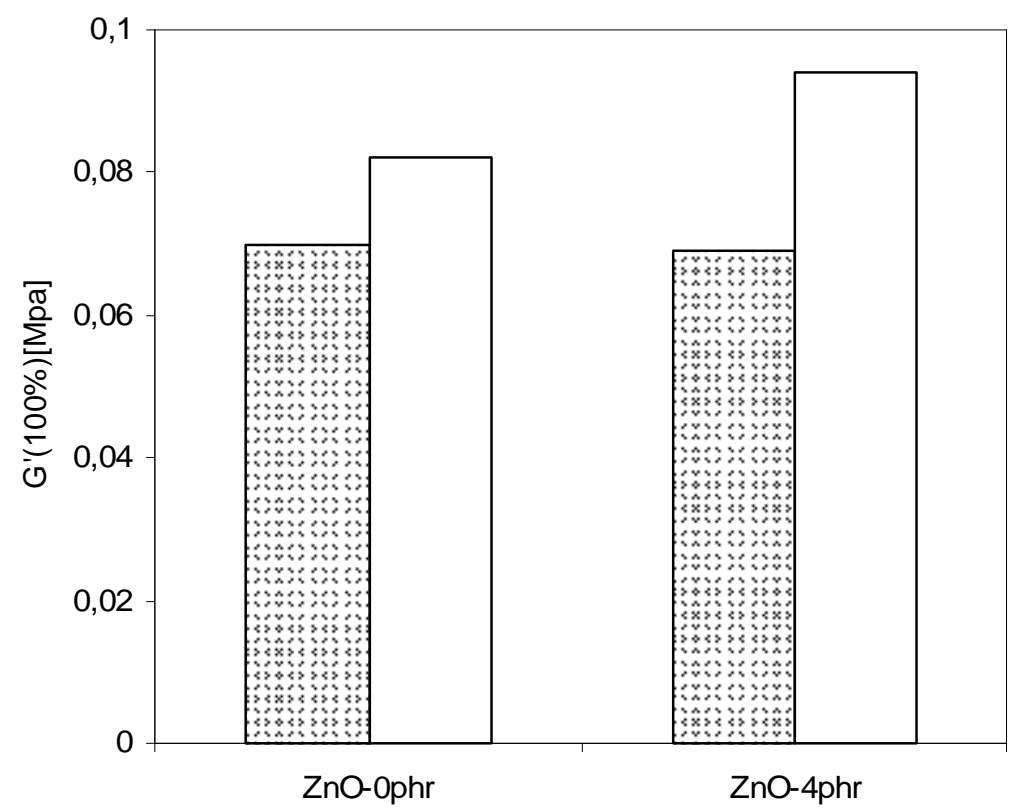

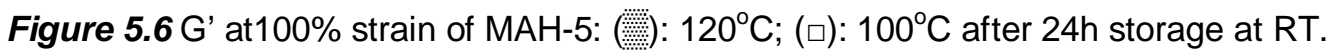

\section{Conclusions}

As demonstrated in Chapter 4, the addition of $5 \mathrm{phr}$ maleic-anhydride grafted EPM to a 50phr HAF-reinforced 35/35/25phr NR/BR/EPDM compound for tyre sidewalls, has a significantly positive effect on the vulcanised mechanical properties. Possible causes for this improvement were systematically investigated. A polarity change of the EPDM-phase after the addition of the MAH-EPM is not significant enough to improve the solubility of curatives in the EPDM phase to account for better curing of the EPDM moiety in the blend. TEM images of gum and HAF-filled NR/BR/EPDM/MAH-EPM blends illustrate a significantly more homogeneous reinforcing carbon black distribution between the various phases in comparison with the straight NR/BR/EPDM blends. Compression set and strain sweep measurements 
further indicate the formation of additional crosslinks in the blends due to the ionic interaction of MAH-EPM with zinc oxide. These ionic crosslinks reversibly decompose at high temperatures above $100^{\circ} \mathrm{C}$.

\section{REFERENCES}

1. Trivedi, B. C.; Culbertson, B. M., in "Maleic Anhdride", Plenum Press, New York, 1982.

2. Moad, G., Prog. in Polym. Sci., 24, 81 (1999).

3. Duin, M. van; Aussems, M.; Borggreve, J. M. R., J. Polym. Sci., Part A, 36, 179 (1998).

4. Machado, A. V.; Covas, J.; Duin, M. van, J. Polym. Sci., Part A, 37, 1311 (1999).

5. Coran, A. Y., Rubber Chem. Technol., 61, 281 (1988).

6. Coran, A. Y., Rubber Chem. Technol., 64, 801 (1991).

7. Morris, M. D.; Thomas, A. G., Rubber Chem. Technol., 68, 794 (1995).

8. Ignatz-Hoover, F.; To, B. H.; Datta, R. N.; Hoog, A. J. d.; Huntink, N. M.; Talma, A. G., Rubber Chem. Technol., 76, 747 (2003).

9. Guillaumond, F. X., Rubber Chem. Technol., 105 (1976).

10. Guo, R.; Talma, A. G.; Datta, R. N.; Dierkes, W. K.; Noordermeer, J. W. M., Europ. Polym. J., 44, 3890 (2008).

11. Sawyer, L. C.; Grubb, D. T., in "Polymer Microscopy", Chapman and Hall, London, 1996.

12. Wouters, M. E. L.; Litvinov, V. M.; Binsbergen, F. L.; Goossens, J. G. P.; Duin, M. van; Dikland, H. G., Macromolecules, 36, 1147 (2003).

13. vandeMee, M. A. J.; Goossens, J. G. P.; Duin, M. van, Rubber Chem. Technol., 81, 96 (2008).

14. Payne, A. R., Rubber Plast. Age., 963 (1961).

15. Gerspacher, M.; O'Farrell, C. P.; Yang, H. H.; Wampler, W. A. Meeting of the Rubber Division, American Chemical Society, Montreal, Canada, 1996.

16. Anthony, P.; De, S. K., J. Macro. Sci., Polymer Reviews, C41, 41 (2001).

17. Bagrodia, S.; Wilkes, G. L.; Kennedy, J. P., Polym. Eng. Sci., 26, 662 (1986). 


\title{
Chapter 6
}

\section{Modification of Maleic Anhydride Grafted EPM with Sulphur Containing Chemicals Aiming at Improved Co-curing}

\begin{abstract}
Maleic anhydride (MAH) grafted EPM can be further modified due to the high chemical reactivity of the MAHunit. Modifying MAH-EPM with sulphur-containing chemicals may help the co-cure in NR/BR/EPDM blends, which is absent with straight MAH-EPM. In this study, CBS and DTDA are studied as modification agents for the MAH-EPM. For CBS, only the cyclohexylamine group is grafted, while the benzothiazole group is decomposed and left unattached. It is further extracted. In the reaction of DTDA-modification onto MAH-EPM, simple modification and crosslinking take place at a same time. With 1.0eq DTDA, relatively less crosslinking and more simple modification is achieved compared to 0.5 eq. The addition of 1.0eq DTDA-modified MAH-EPM to the NR/BR/EPDM blend does not give a substantial improvement of the tensile properties as compared to unmodified MAH-EPM.
\end{abstract}




\subsection{Introduction}

MAH-grafted polyolefins, such as polyethylene (PE), polypropylene (PP), ethylene propylene copolymers (EP[D]M) and hydrogenated styrene-isoprenestyrene block copolymers (SEBS), have been studied, produced and applied since the 1950s. They have found widespread applications as reactive compatibilisers or adhesives, as a result of their increased polarity and reactivity, for apolar polyolefins to polar materials ${ }^{1-4}$.

In previous studies as already discussed in Chapters 4 and 5, MAH-EPM was added to NR/BR/EPDM blends as compatibilising agent. The mechanical properties of such blends were improved by the addition of $5 \mathrm{phr}$ of MAH-EPM, resulting in increased tensile strength, higher modulus, better trouser tear strength and increased fatigue life based on constant strain energy. Those improvements can mainly be attributed to a compatibilising effect of MAH-EPM between EPDM and NR/BR, resulting in a more homogeneous phase morphology and better carbon black distribution, as well as to some ionic crosslinks introduced into the blends by the reaction of $\mathrm{MAH}-\mathrm{EPM}$ and $\mathrm{ZnO}$. However, due to the completely saturated chemical structure of MAH-EPM, there is no possibility for co-curing between MAH-EPM and the other rubbers by simply applying this MAH-EPM to the blend. Therefore, if this problem could be solved, more improvements can be expected for the NR/BR/EPDM/MAH-EPM blends.

Due to the high chemical reactivity of the grafted MAH unit, MAH-modified polyolefins can be further modified with a wide range of other functionalities, for instance amines, amides, alcohols, oxazoline, isocyanates and metal oxides. A primary or a secondary amine can be used to open the anhydride ring, thereby forming amide-acids as shown in scheme $6 \cdot 1^{5-7}$. The initially-formed primary amideacids can undergo further reaction to form imides at elevated temperatures. By reacting sulphur-containing agents having amine or other reactive functionalities for the MAH unit, onto MAH-EPM may help to enhance co-curing between MAH-EPM and sulphur-curing rubbers.

In this chapter, modification of MAH-EPM with $\mathrm{N}$-cyclohexyl- 2benzothiazolesulfenamide (CBS) and dithiodianiline (DTDA) as sulphur-containing agents will be described. FTIR-ATR and NMR are used to characterize the modification reactions. Mechanical properties of the NR/BR/EPDM blend with modified MAH-EPM are studied as well. 


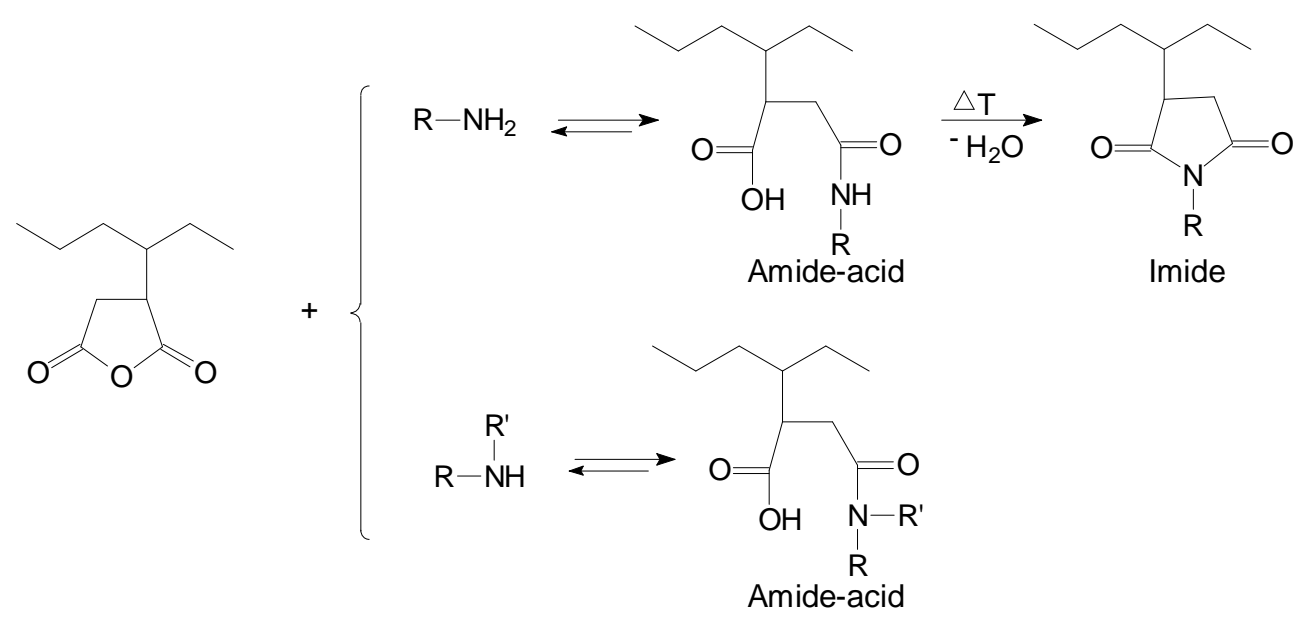

Scheme 6.1 Reaction of maleic anhydride with primary and secondary amines.

\subsection{Experimental}

\subsubsection{Materials}

Maleic anhydride grafted EPM (MAH-EPM, 49 wt\% ethylene, 49 wt\% propylene, 2.0 wt\% maleic anhydride) was provided by DSM Elastomers B.V., the Netherlands. It was dried for 1 hour at $180^{\circ} \mathrm{C}$ in a vacuum oven before use in order to convert any carboxylic acid groups back into the anhydride form.

$\mathrm{N}$-cyclohexyl-2-benzothiazolesulfenamide (CBS) or Santocure ${ }^{\circledR}$ CBS (Flexsys B.V., the Netherlands), 4,4'-dithiodianiline (98\%, DTDA) (Aldrich) were used as modification agents.

The elastomers selected for the blend were natural rubber (SIR20, Standard Indonesian Rubber), butadiene rubber (Kosyn KBR01, Korea Kumho Petrochemical Co., Ltd.) and EPDM rubber (Keltan 5508, DSM Elastomers B.V., the Netherlands) containing $4.5 \mathrm{wt} \%$ of ethylidene norbornene (ENB) and $70 \mathrm{wt} \%$ of ethylene.

The other compounding ingredients used were extra pure grade zinc oxide (Merck, Germany), finely divided sulphur (Merck, Germany), 95\% pure stearic acid (Aldrich, Germany), poly(2,2,4-trimethyl-1,2-dihydroquinoline) or TMQ (Flexsys B.V., the Netherlands). High abrasion furnace black (HAF-N330, Cabot Corporation) and naphthenic oil Sunthene ${ }^{\circledR} 4240$ (Sun Petroleum Products Co., Ltd.) were used in the compounds.

\subsubsection{Modification reactions}

The modification agent, such as CBS or DTDA, was added to MAH-EPM on a Schwabenthan two-roll mill. The amounts of CBS and DTDA were varied corresponding to the molar amount of MAH-groups in the MAH-EPM, as shown in 
Table 6.1. The blend of MAH-EPM with CBS or DTDA was then heated in a compression molding machine (Wickert WLP1600 laboratory press) at 100 bar at $140^{\circ} \mathrm{C}$ for 10 minutes. The reaction products were then extracted by acetone for 24 hours to remove unreacted chemicals before characterization.

Table 6.1 Recipe for MAH-EPM modification with CBS and DTDA.

\begin{tabular}{c|cccc}
\hline Ingredients & 0eq & $0.5 \mathrm{eq}$ & $1.0 \mathrm{eq}$ & $1.5 \mathrm{eq}$ \\
\hline MAH-EPM & \multicolumn{4}{|c}{$100 \mathrm{~g}(2 \mathrm{~g} \mathrm{MAH}=0.02 \mathrm{~mol})$} \\
CBS & 0 & $2.64 \mathrm{~g}$ & $5.28 \mathrm{~g}$ & $7.92 \mathrm{~g}$ \\
$(264 \mathrm{~g} / \mathrm{mol})$ & 0 & $(0.01 \mathrm{~mol})$ & $(0.02 \mathrm{~mol})$ & $(0.03 \mathrm{~mol})$ \\
DTDA & & $2.48 \mathrm{~g}$ & $4.96 \mathrm{~g}$ & - \\
$(248 \mathrm{~g} / \mathrm{mol})$ & 0 & $(0.01 \mathrm{~mol})$ & $(0.02 \mathrm{~mol})$ & - \\
\hline
\end{tabular}

\subsubsection{Characterization of the MAH-grafted and modified EPM-samples}

ATR-IR - The infrared spectra of the MAH-grafted and modified EPM-samples were recorded by using the attenuated total reflectance infrared technique (ATR/IR) with a Perkin Elmer Spectrum 100 IR-spectrometer. The infrared spectra were recorded with 12 scans at a resolution of $4 \mathrm{~cm}^{-1}$ in the range of $4000-600 \mathrm{~cm}^{-1}$.

NMR - The 1H-NMR spectra of the grafted EPM-samples were recorded with a Varian Unity $300 \mathrm{MHz}$ system at $25^{\circ} \mathrm{C}$ with $\mathrm{CDCl}_{3}$ as the solvent.

\subsubsection{Preparation of the blends, vulcanisation and tensile tests}

Blend compounds with DTDA-modified-MAH-EPM were prepared in a Brabender Plasticorder 350 S mixer having a mixing chamber volume of $370 \mathrm{~cm}^{3}$. The mixer was operated at a rotor speed of $80 \mathrm{rpm}$, fill factor of 0.7 and an initial temperature of $50^{\circ} \mathrm{C}$. The recipes are shown in Table 6.2. NR was first masticated for 2 minutes, then BR, EPDM, MAH-EPM or DTDA-modified-MAH-EPM were added and mixed for 1 more minute. HAF-black, oil, $\mathrm{ZnO}$, stearic acid and TMQ were subsequently added and the mixing was continued for another 2 minutes. The final compound temperature before dumping from the mixer was in the range of 110 $115^{\circ} \mathrm{C}$. Curing additives CBS and sulphur were added into the compound on a tworoll mill. The resulting compound was tested for its cure characteristics using a RPA 2000 curemeter of Alpha Technologies at $140^{\circ} \mathrm{C}$ according to ISO 6502. 
Table 6.2 Formulation of NR/BR/EPDM compounds

\begin{tabular}{l|ccc}
\hline \multicolumn{1}{c|}{ Ingredients } & \multicolumn{3}{c}{ Contents (phr) } \\
\hline & Ref-1 & MAH-5 & D-MAH-5 \\
\hline NR & 35 & 35 & 35 \\
BR & 35 & 35 & 35 \\
EPDM & 30 & 25 & 25 \\
MAH-EPM & - & 5 & - \\
DTDA-MAH-EPM & - & - & 5.25 \\
(1.0eq DTDA modified) & & & \\
HAF N330 & 50 & 50 & 50 \\
Sunthene 4240 & 10 & 10 & 10 \\
Stearic acid & 2 & 2 & 2 \\
Zinc Oxide & 4 & 4 & 4 \\
TMQ & 1 & 1 & 1 \\
CBS & 1.98 & 1.98 & 1.98 \\
Sulphur & 2.5 & 2.5 & 2.5 \\
\hline
\end{tabular}

The optimum cure time $\left(t_{c} 90\right)$ of the fully compounded blends was determined as the time needed to reach $90 \%$ of the maximum torque in the RPA 2000 at $140^{\circ} \mathrm{C}$, $0.833 \mathrm{~Hz}$ and 0.2 degree strain. The blends were then vulcanized for the optimum cure time $t_{c} 90$ in a Wickert WLP1600 laboratory compression press at $140^{\circ} \mathrm{C}$ and 100 bar.

Tensile tests were carried out according to ISO 37, with a Zwick tensile tester Model Z 1.0/TH1S at a constant crosshead speed of $500 \mathrm{~mm} / \mathrm{min}$.

\subsection{Results and Discussion}

\subsubsection{Modification of MAH-EPM with CBS}

CBS is one of the most commonly used accelerators for rubber compounds. Because of the secondary amine group in the structure of CBS, it is able to react with $\mathrm{MAH}$-EPM and form an amide.

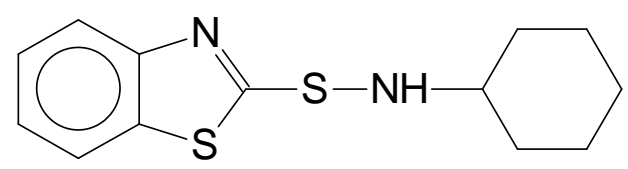

Scheme 6.2 Chemical structure of CBS. 
Figure 6.1 shows the ATR/IR spectra of the original MAH-EPM and grafted MAHEPM with different amounts of CBS. The unmodified MAH-EPM shows two absorption bands at $1862 \mathrm{~cm}^{-1}$ and $1782 \mathrm{~cm}^{-1}$, which can be attributed to the antisymmetric and symmetric $\mathrm{C}=\mathrm{O}$ stretching vibrations of saturated, cyclic anhydride groups. After reaction with CBS, three additional bands appear. The band around $1710 \mathrm{~cm}^{-1}$ is assigned to the $\mathrm{C}=\mathrm{O}$ stretching vibration of the carboxylic acid. The other two bands at $1640 \mathrm{~cm}^{-1}$ and $1550 \mathrm{~cm}^{-1}$ are from the $\mathrm{C}=\mathrm{O}$ and $\mathrm{N}-\mathrm{H}$ stretching vibrations of the amide ${ }^{6,8,9}$. The presence of above absorption peaks indicates that amide-acid is formed. By changing the CBS amounts, the intensities of the anhydride-bands decrease with higher CBS amounts and the intensities of the bands at $1710 \mathrm{~cm}^{-1}, 1640 \mathrm{~cm}^{-1}$ and $1550 \mathrm{~cm}^{-1}$ increase.

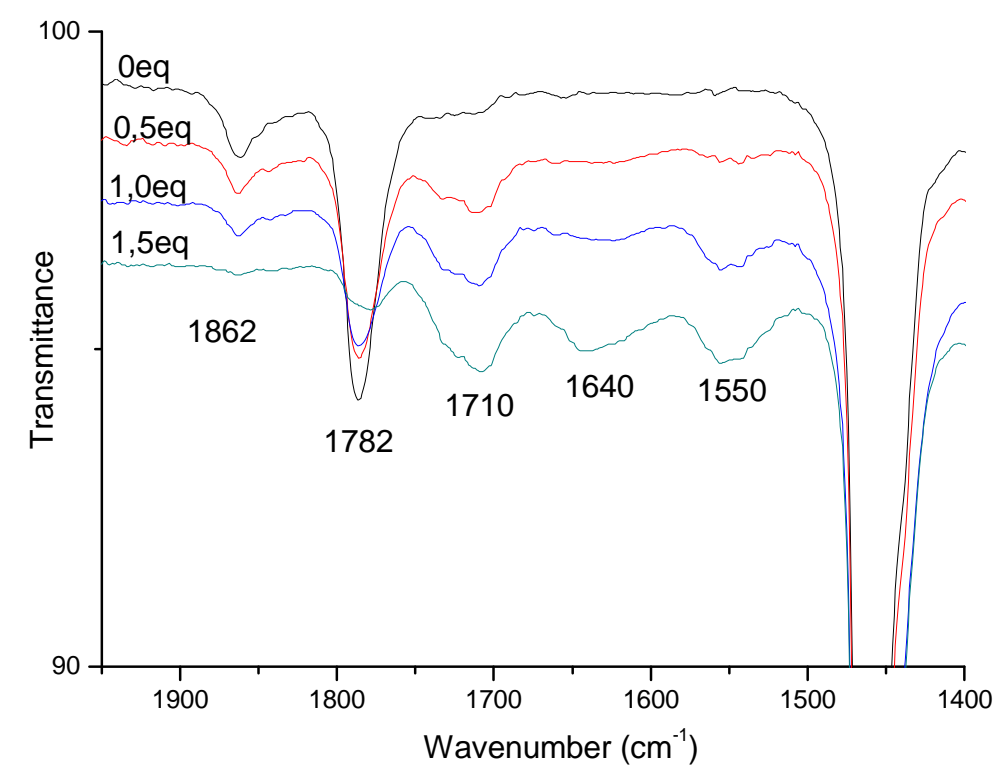

Figure 6.1 ATR/IR spectra of MAH-EPM modified with different amounts of CBS.

Table 6.3 Assignment of the characteristic $\mathrm{C}=\mathrm{O}$ and $\mathrm{N}-\mathrm{H}$ bands in the ATR/IR spectra for CBS-modified MAH-EPM.

\begin{tabular}{cc}
\hline Wavenumber $\left(\mathrm{cm}^{-1}\right)$ & Assignment \\
\hline 1862 & (symmetric) anhydride $\mathrm{C}=\mathrm{O}$ \\
1782 & (anti-symmetric) anhydride $\mathrm{C}=\mathrm{O}$ \\
1710 & Carboxylic acid C=O \\
1640 & Amide $\mathrm{C}=\mathrm{O}$ \\
1550 & Amide N-H \\
\hline
\end{tabular}

If CBS simply reacted with the maleic anhydride group as a secondary amine as shown in scheme 6.1, a tertiary amide would be formed and no amide $\mathrm{N}-\mathrm{H}$ group 
would present in the products. Moreover, the benzothiazole group would graft onto MAH-EPM as well. However, no clear band proves the presence of such benzothiazole groups in the ATR spectra after reaction.

In order to clarify this further, NMR spectra were measured. In the NMR spectrum of CBS, several peaks in the range from 7.2ppm to $8.0 \mathrm{ppm}$ can clearly be assigned to aromatic protons; the peaks from $0.6 \mathrm{ppm}$ to $2.2 \mathrm{ppm}$ can be attributed to the protons of the cyclohexyl group; two peaks at $2.9 \mathrm{ppm}$ and $3.25 \mathrm{ppm}$ are most probably from amine protons. In the spectrum of CBS-modified MAH-EPM, an extra small peak at 2.45ppm is observed compared to the original MAH-EPM. However, no peak from aromatic protons is observed in CBS-modified MAH-EPM, confirming that the benzothiazole group was not grafted onto MAH-EPM. Therefore, after the reaction of the amine group and maleic anhydride, only the cyclohexylamine group was grafted in the form of an amide, while the benzothiazole group was separated from the CBS and left in some possible form, as mercapto-benzothiazol or dithiobis(benzothiazole). The unattached benzothiazols is extracted in the acetoneextraction, taking along all sulphur moieties which otherwise would have been beneficial for co-crosslinking of the MAH-EPM. The modified MAH-EPM is then left just similar to the N-phenyl-p-phenylenediamine (NPPDA) modified MAH-EPM as already discussed in Chapter 4 and does not give improvement of the mechanical properties for the NR/BR/EPDM blends. This route was therefore not further pursued for mechanical testing.

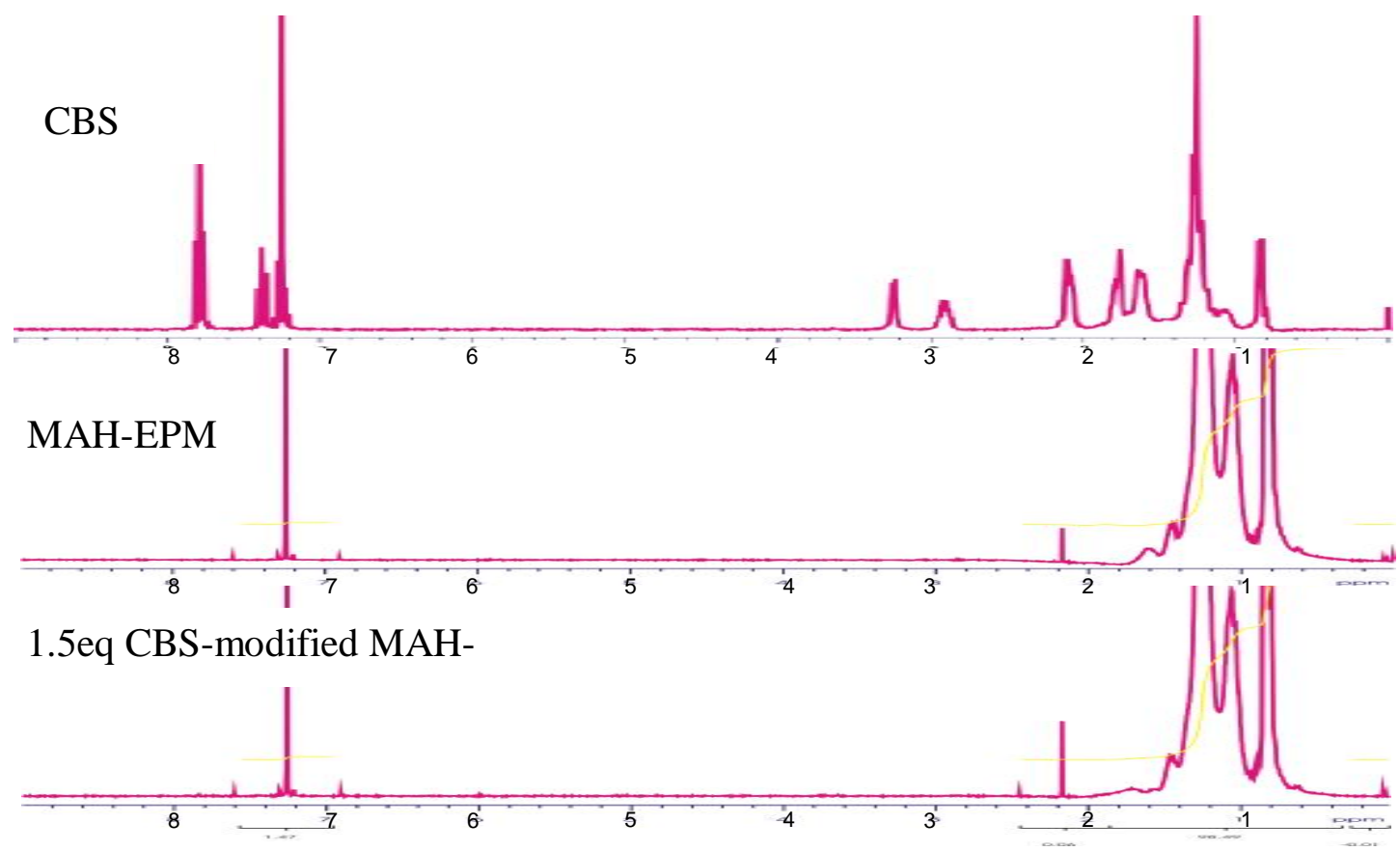

Figure 6.2 NMR spectra of CBS, MAH-EPM and 1.5eq CBS-modified MAH-EPM. 


\subsubsection{Modification of MAH-EPM with DTDA}

From its chemical structure as shown in scheme 6.3, it can be seen that DTDA has amine groups, which can react with anhydride functionalities, and di-sulphur in the middle which can introduce sulphur co-cure for MAH-EPM with the other rubbers.

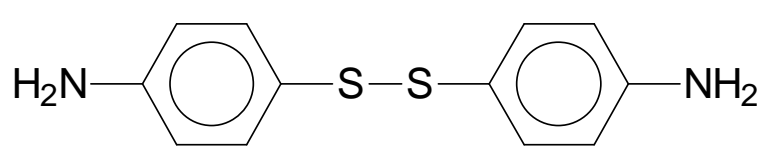

Scheme 6.3 Chemical structure of DTDA.

Figure 6.3 shows the ATR/IR spectra of DTDA and DTDA-modified MAH-EPM in comparison with the original MAH-EPM. DTDA-modified MAH-EPM clearly shows some additional bands which originate from DTDA. For example, the band at $1588 \mathrm{~cm}^{-1}$ is from the Ar- $\mathrm{NH}_{2}$ group, and the band at $822 \mathrm{~cm}^{-1}$ can be attributed to the aromatic ring. In addition, after reaction with DTDA, two anhydride-bands which exist in the original MAH-EPM disappear and one band at $1708 \mathrm{~cm}^{-1}$ appears which can be assigned to the anti-symmetric $\mathrm{C}=\mathrm{O}$ stretching vibration of an imide instead of amide, because no amide bands at $1640 \mathrm{~cm}^{-1}$ and $1550 \mathrm{~cm}^{-1}$ are observed ${ }^{6,7,10}$. A new band appears at $1400 \mathrm{~cm}^{-1}$ confirming the formation of the imide, which originates from the $\mathrm{C}-\mathrm{N}$ stretching of the imide ring ${ }^{11,12}$. Therefore, DTDA-fragments are indeed grafted onto MAH-EPDM and the imide is formed at $140^{\circ} \mathrm{C}$. Comparing the spectrum for 0.5eq DTDA-modified and 1.0eq DTDA-modified MAH-EPM, it is clear that the amount of DTDA does not significantly influence the intensity of the above bands. After reaction, the modified MAH-EPM becomes insoluble in chloroform, indicating that some intermolecular linkages are formed. 


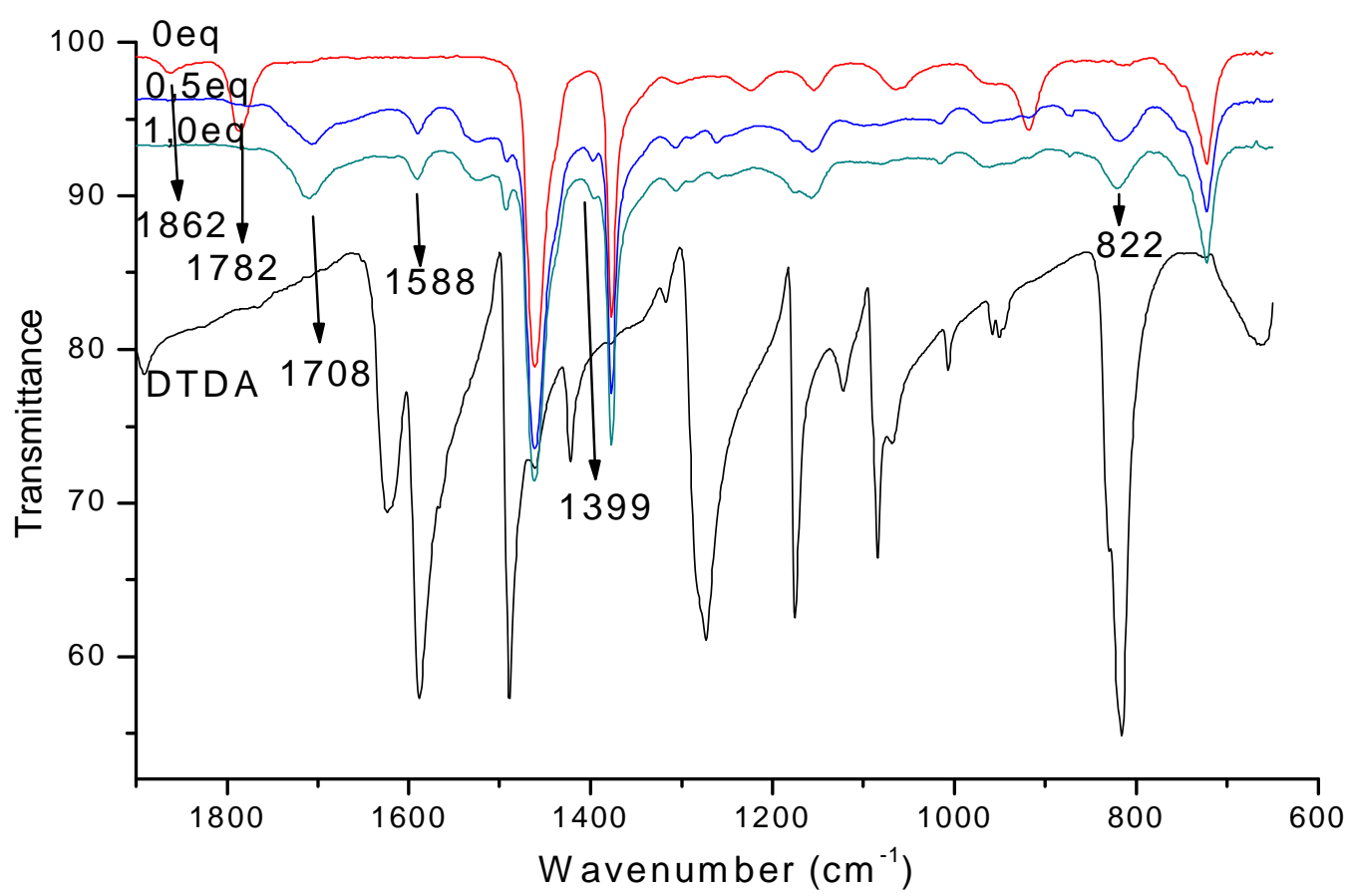

Figure 6.3 ATR/IR spectra of DTDA and MAH-EPM modified with different amounts of DTDA.

Table 6.4 Assignment of the bands in the ATR/IR spectra for DTDA-modified MAH-EPM.

\begin{tabular}{cc}
\hline Wavenumber $(\mathrm{cm}-1)$ & Assignment \\
\hline 1862 & (symmetric) anhydride $\mathrm{C}=\mathrm{O}$ \\
1782 & (anti-symmetric) anhydride $\mathrm{C}=\mathrm{O}$ \\
1708 & (anti-symmetric) imide $\mathrm{C}=\mathrm{O}$ \\
1588 & Amine Ar-NH \\
1399 & imide C-N \\
822 & Aromatic ring \\
\hline
\end{tabular}

Simple unreacted blends of MAH-EPM and DTDA were tested in the RPA instrument at $140^{\circ} \mathrm{C}$. As shown in figure 6.4 , the torque values increases with time for both blends with $0.5 \mathrm{eq}$ and $1.0 \mathrm{eq}$ DTDA, respectively. This indicates that a certain amount of crosslinking occurs during the modification reaction. The blend of $\mathrm{MAH}-$ EPM with 0.5eq DTDA shows a higher torque value than the other one with 1.0eq DTDA, indicating a higher crosslink density. As each DTDA-molecule has two amine groups, 0.5eq DTDA has $1.0 \mathrm{eq}$ amine groups indeed. Therefore, with 0.5eq DTDA, all of its amine groups can react with anhydride groups in principle. In other words, one DTDA-molecule may react with two anhydride groups and form the highest extent of crosslinking. For $1.0 \mathrm{eq}$ DTDA, there are $2.0 \mathrm{eq}$ amine groups and only half 
of the amine groups can react with anhydride. Idealy, in this case, one DTDAmolecule may only react with one anhydride group as simple-modification and there will be no intermolecular linkage taking place. Crosslinking and simple grafting practically co-exist, despite the DTDA-amount, as shown in scheme 6.4. However, the blend with 1.0eq DTDA shows relatively more simple-grafting and less crosslinking as compared to the one with 0.5eq DTDA. In either case, the MAH-units are modified with sulphur-containing moieties which might help in co-curing with the various rubbers. Mechanical testing was further pursued with the 1.0eq DTDAmodified EPM, because it was considered to be lower in the relative amount of internal crosslinking.

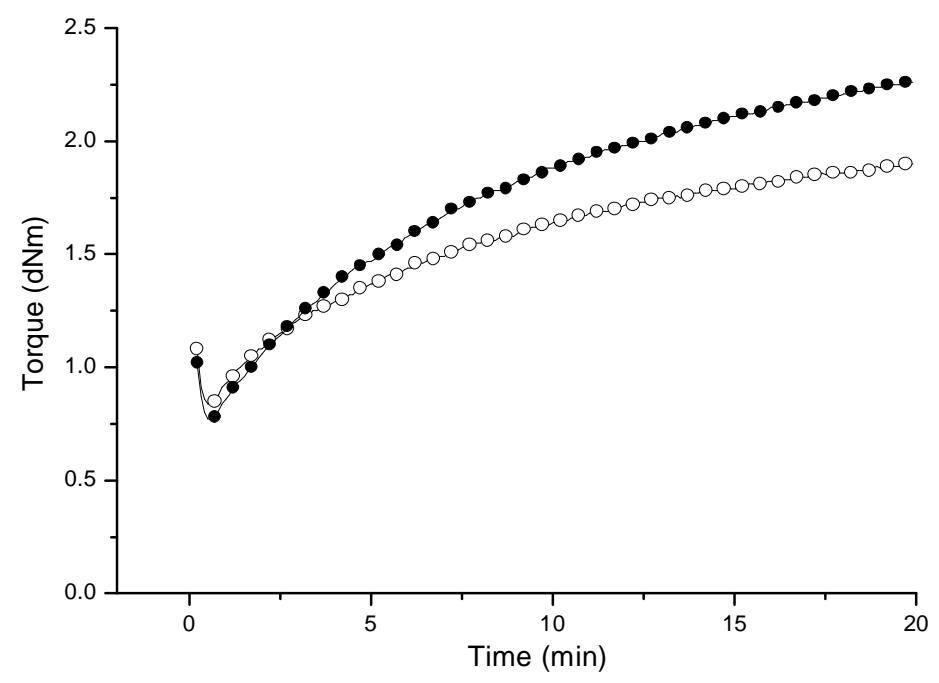

Figure 6.4 Torque change during the modification reactions between DTDA and MAH-EPM: $(\bullet-\bullet)$ with 0.5eq DTDA; (०—०) with 1.0eq DTDA.

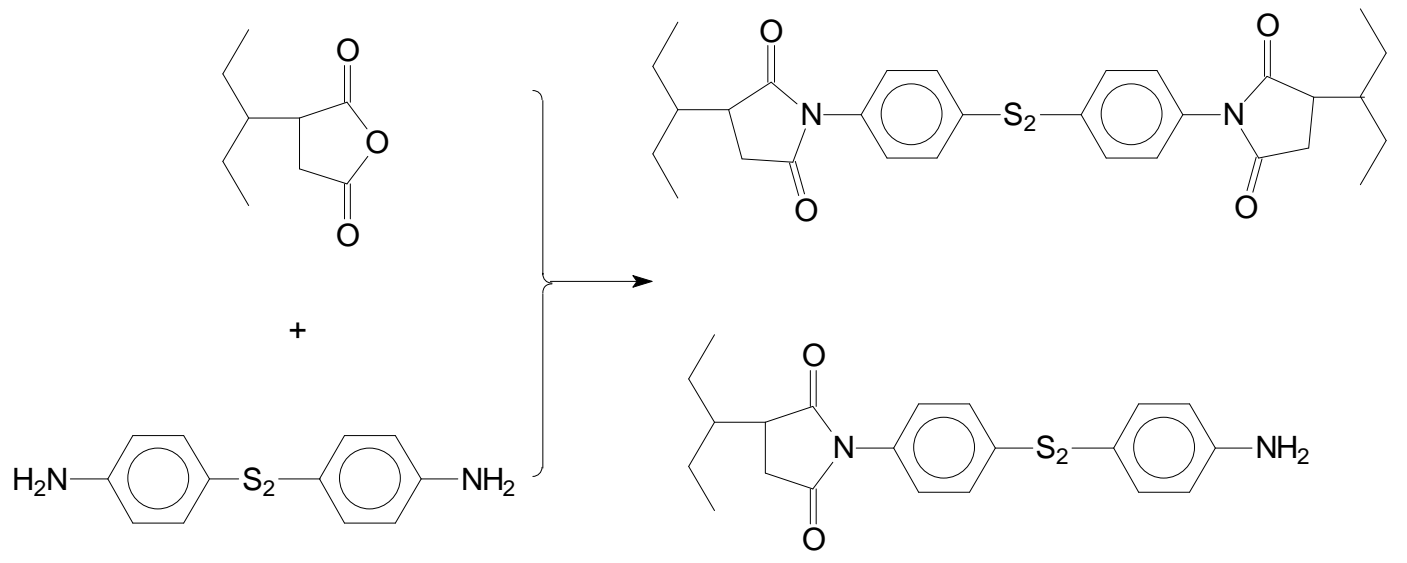

Scheme 6.4 Possible reactions for DTDA with MAH-EPM. 


\subsubsection{DTDA modified-MAH-EPM in NR/BR/EPDM blends}

The 1.0eq DTDA modified MAH-EPM was then blended with NR/BR/EPDM according to the formulation in Table 6.2. From the curing curves as shown in figure 6.5 , it is evident that the addition of MAH-EPM results in some scorch delay, which comes from a well-known retarding effect of the maleic anhydride and eventual carboxylic acid. The blend with DTDA-modified MAH-EPM has a similar scorch time as the straight NR/BR/EPDM blend, shorter than that of the blend with unmodified MAH-EPM, because of less or no maleic anhydride and carboxylic acid present after the modification. With respect to the maximum torque value, the addition of $\mathrm{MAH}$ EPM leads to some higher maximum torque value compared to Ref- 1 . The DTDAmodified MAH-EPM shows even a higher maximum torque value, indicating more crosslinking introduced into the blend by the DTDA-modification of the MAH-EPM, so that it can participate in the crosslinking process.

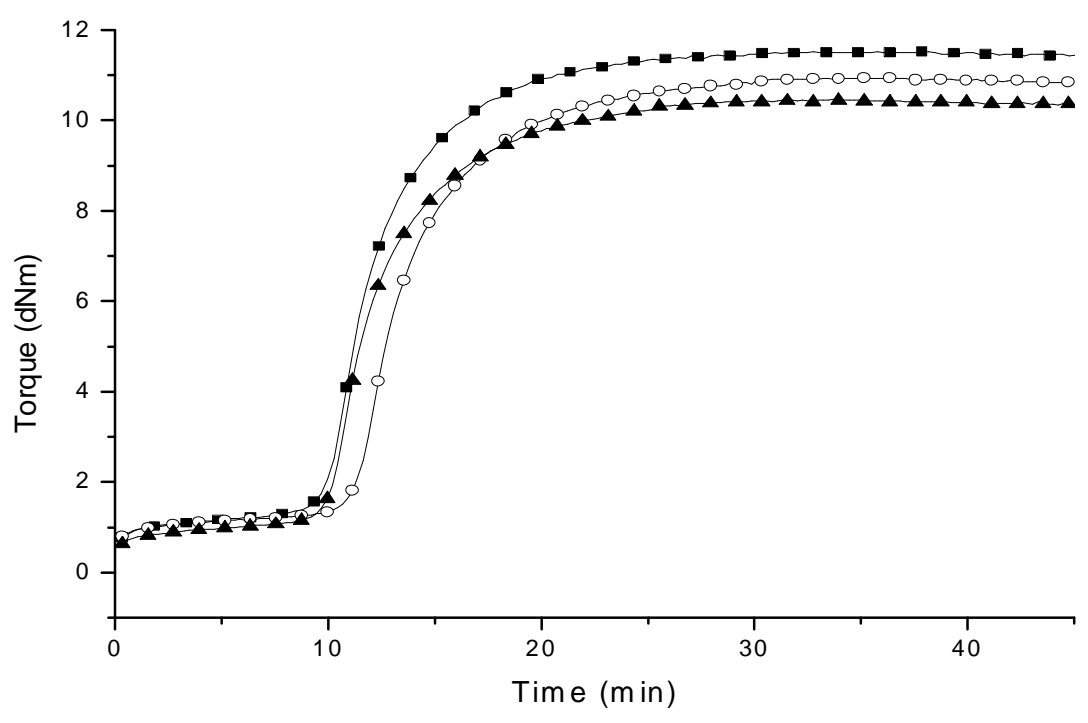

Figure 6.5 RPA curing curves for NR/BR/EPDM and NR/BR/EPDM/MAH-EPM or modified

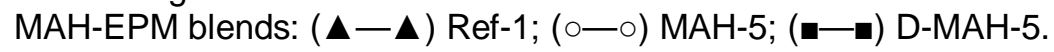

Figure 6.6 shows representative tensile curves for the three compounds after curing. It is clear that the addition of MAH-EPM does already improve the tensile properties compared to the straight NR/BR/EPDM blend as higher modulus, higher tensile strength and increased elongation at break. By using DTDA-modified MAH$E P M$, the modulus increases even more, consistent with the maximum torque value in the curing curve. However, the elongation at break decreases somewhat in this case, and the tensile strength is more or less the same as for unmodified MAH-EPM. 


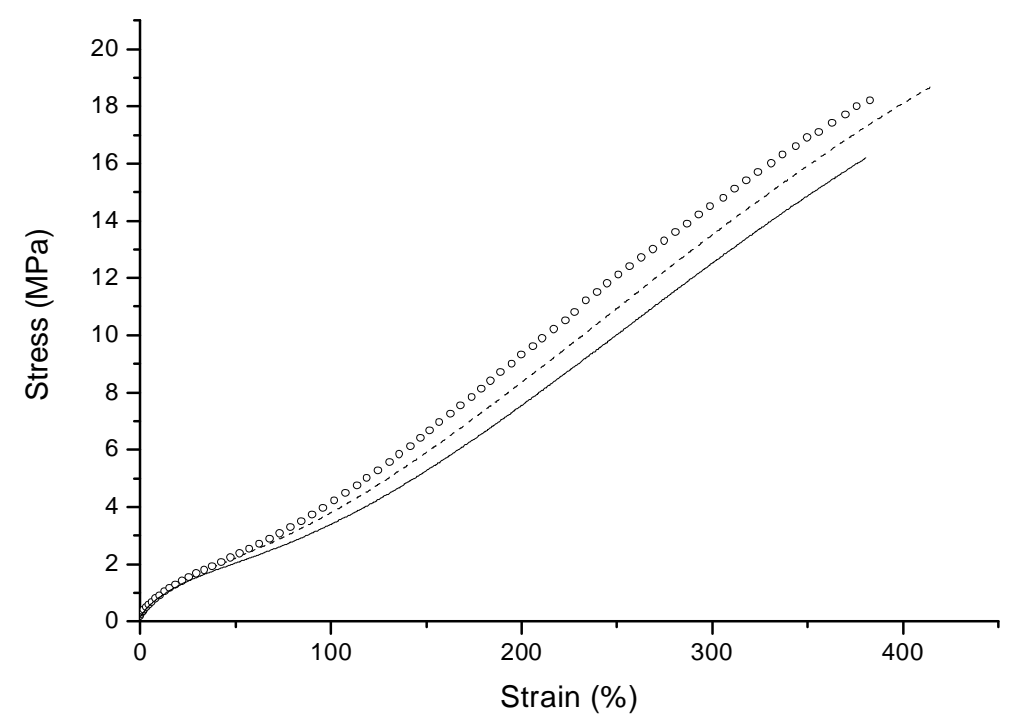

Figure 6.6 Tensile curves for NR/BR/EPDM and NR/BR/EPDM/MAH-EPM (modified MAHEPM) blends: (一) Ref-1; (---) MAH-5; (마) D-MAH-5.

Therefore, with DTDA-modified MAH-EPM, no substantial further improvement of the tensile properties is observed compared to MAH-EPM. As already discussed in previous chapters, the addition of straight MAH-EPM into NR/BR/EPDM blends brings already a compatibilising effect, resulting in a more homogeneous phase and better carbon black distribution, and additional ionic crosslinks in the MAH-EPM phase. For DTDA-modified MAH-EPM, the higher modulus for the final compounds can be attributed to some more crosslinking by the modification. However, because some of this additional crosslinking may already have happened during the modification reaction, the partly crosslinked modified MAH-EPM may result in a poor distribution in the blends. The co-cure effect by the disulphide group in the modified MAH-EPM is then limited, if not completely disturbed.

\subsection{Conclusions}

During the modification reaction of CBS with MAH-EPM, CBS is not grafted onto MAH-EPM as one complete molecule. The benzothiazole group in CBS, which would help with the co-cure of MAH-EPM with the other rubbers in the blends, was not grafted. Only the cyclohexylamine group was grafted and an acid-amide was formed. Then the modified MAH-EPM will not improve the mechanical properties, as seen in the earlier studies on NPD-EPM in Chapter 4.

For DTDA-modification onto MAH-EPM, 0.5eq and 1.0eq DTDA have been tried, respectively. In both cases simple modification and crosslinking takes place. With 1.0 eq DTDA, relatively less crosslinking and more straight modification can be 
achieved. The addition of 1.0eq DTDA-modified MAH-EPM to the NR/BR/EPDM rubber blend does not give a substantial improvement of the tensile properties compared to the use of straight MAH-EPM.

\section{REFERENCES}

1. Trivedi, B. C.; Culbertson, B. M., "Maleic Anhdride", Plenum Press, New York, 1982.

2. Moad, G., Prog. in Polym. Sci., 24, 81 (1999).

3. van Duin, M.; Aussems, M.; Borggreve, J. M. R., J. Polym. Sci., Part A, 36, 179 (1998).

4. Machado, A. V.; Covas, J.; van Duin, M., J. Polym. Sci., Part A, 37, 1311 (1999).

5. Schmidt, U.; Zschoche, S.; Werner, C., J. Appl. Polym. Sci., 87, 1255 (2003).

6. Padwa, A. R.; Macosko, C. W.; Wolske, K. A.; Sasaki, Y., Polym. Prepr., 34, 842 (1993).

7. Hu, G. H.; Lindt, J. T., Polym. Bull, 29, 357 (1992).

8. Bayer, T.; Eichhorn, K. J.; Grundke, K.; Jacobasch, H. J., Macromol. Chem. Phys., 200, 852 (1999).

9. Vermeesch, I.; Groeninckx, G., J. Appl. Polym. Sci., 53, 1365 (1994).

10. Lee, S.-S.; Ahn, T. O., J. Appl. Polym. Sci., 71, 1187 (1999).

11. Pryde, C. A., J. Polym. Sci., Part A, 27, 711 (1989).

12. Insberg, R. G.; Susko, J. R., in "Polyimides", Plenum Press, New York, 1984. 


\section{Chapter 7}

\section{Studies on EPDM Modification by CBS, PNO and CLD}

In order to solve the cure incompatibility and heterogeneous carbon black distribution in the NR/BR/EPDM blend system for tyre sidewalls, Ncyclohexyl-2-benzothiazolesulphenamide (CBS), 2,2'-dithiobis(pyridine-N-oxide) (PNO) and caprolactam disulfide (CLD) have been studied as modification agents to make EPDM more reactive towards curing and more attractive to fillers. Different compositions, temperatures and times have been tried for the modification reactions. CBS fragments have been found to be grafted onto EPDM in the presence of sulphur and $\mathrm{ZnO}$. However, both PNO and CLD are very sensitive to become crosslinked during the reaction, and further no evidence from ATR/IR and NMR shows that grafting actually takes place. 


\subsection{Introduction}

In order to improve the mechanical properties of EPDM/high-diene rubber blends, modifying EPDM to make it more reactive towards curing and more attractive to fillers is one of the possible approaches. In this chapter, N-cyclohexyl-2benzothiazolesulfenamide (CBS), 2,2'-dithiobis(pyridine-N-oxide) (PNO) and caprolactam disulfide (CLD) are studied as modification agents because of their special chemical structures: containing S-S or S-N bonds, as shown in scheme 7.1.

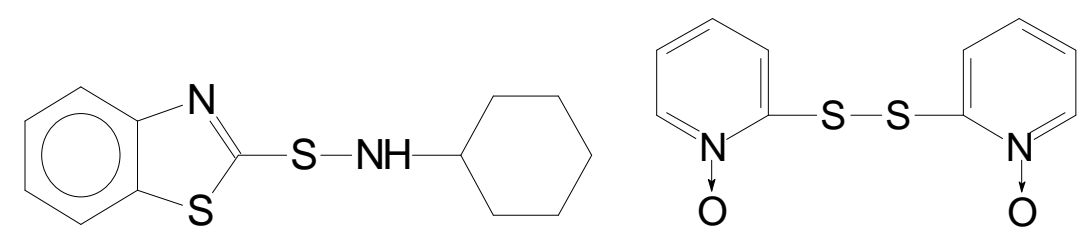

(a)

(b)

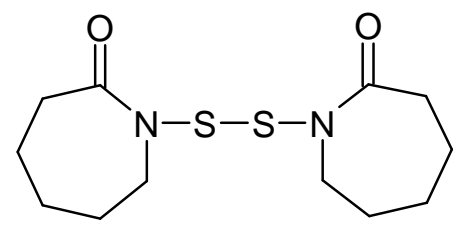

(c)

Scheme 7.1 Chemical structures of (a) CBS; (b) PNO; (c) CLD.

CBS is a commonly used accelerator for rubber vulcanisation. Sahakaro ${ }^{1-3}$ et al. used a reactive processing method to prepare NR/BR/EPDM blends. In that method, EPDM was first pretreated with all the curatives and additives, including sulphur, CBS, stearic acid and ZnO. The pretreated EPDM was then mixed with premasticated NR/BR. The reactive blend vulcanisates showed a significant improvement in mechanical properties as compared to those prepared by straight mixing. In the pretreatment step, it was believed that accelerator fragments were grafted onto EPDM according to characterisation by infrared spectroscopy.

It has been found that PNO and its zinc salt, zinc-2-mercaptopyridine $\mathrm{N}$-oxide (ZPNO), can be used as accelerators or secondary accelerators in sulphur-cured natural rubber compounds ${ }^{4,5}$. Datta and co-workers developed a vulcanisation process with derivatives of mercaptopyridine as carbon black/natural rubber coupling agents and found that ZPNO is a good carbon black/natural rubber coupling agent ${ }^{6}$. Therefore, if PNO or its zinc salt can be grafted onto EPDM, more carbon black may be attracted into the EPDM phase in blends with high-diene rubbers.

CLD is known as non-nitrosamine forming sulphur donor for the vulcanisation of natural and synthetic rubbers. The benefit of using CLD is that compared to normal sulphur, it forms mono- and disulphidic bridges which results in a better heat ageing 
resistance of the vulcanisates. The grafting of CLD onto EPDM may also introduce higher polarity and reactivity to solve the cure incompatibility and heterogeneous carbon black distribution problems in blends with high-diene rubbers.

In this chapter, the grafting of CBS onto EPDM is first studied on the basis of Sahakaro's work. After that, the reactions of EPDM with PNO and CLD are described, respectively. The characterisations and the mechanisms of the reactions are discussed in this chapter as well.

\subsection{Experimental}

\subsubsection{Materials}

Modification agents are N-cyclohexyl-2-benzothiazolesulphenamide (CBS) or Santocure ${ }^{\circledR}$ CBS (Flexsys B.V., the Netherlands), 2,2'-dithiobis(pyridine-N-oxide) (PNO) (Acros organics, Belgium), caprolactam disulfide (CLD) or Isogran ${ }^{\circledR}$ S-80 (Isogran $\mathrm{GmbH}$, Germany).

EPDM rubber (Keltan 5508, DSM Elastomers B.V., the Netherlands) contains $4.5 \mathrm{wt} \%$ ethylidene norbornene (ENB) as third monomer and $70 w t \%$ of ethylene.

The other ingredients used are extra pure grade zinc oxide (Merck, Germany), finely divided sulphur (Merck, Germany), 95\% pure stearic acid (Aldrich, Germany).

\subsubsection{Modification reactions}

Table 7.1 shows different formulations for EPDM modification with CBS, PNO and CLD. All the compounds were mixed on a Schwabenthan two-roll mill. The blends were then heated in a compression molding machine (Wickert WLP1600 laboratory press) at 100 bar at different temperatures and for different times as shown in Table 7.2. The products were then extracted with acetone for 24 hours to remove unreacted chemicals before characterisation.

Table 7.1 Recipes for EPDM modification with CBS, PNO and CLD.

\begin{tabular}{c|ccc|ccc|cc}
\hline & CBS-1 & CBS-2 & CBS-3 & PNO-1 & PNO-2 & PNO-3 & CLD-1 & CLD-2 \\
\hline EPDM & 100 & 100 & 100 & 100 & 100 & 100 & 100 & 100 \\
CBS & 2 & 2 & 2 & - & - & - & - & - \\
PNO & - & - & - & 2 & 2 & 2 & - & - \\
CLD & - & - & - & - & - & - & 2 & 2 \\
Sulphur & 2.5 & - & 0.3 & - & 0.3 & 0.3 & - & - \\
ZnO & 4 & 0.6 & 0.6 & 0.6 & - & 0.6 & - & 0.6 \\
Stearic acid & 2 & - & - & - & - & - & - & - \\
\hline
\end{tabular}


Table 7.2 Reaction temperatures and times for all the compounds.

\begin{tabular}{|c|c|c|c|c|c|c|}
\hline & CBS-1 & CBS-2 & CBS-3 & PNO-1 & PNO-2 & CLD-1 \\
\hline $\begin{array}{c}\text { Temperature } \\
\left({ }^{\circ} \mathrm{C}\right)\end{array}$ & 140 & 160 & 160 & for all: & $: 160,170,180$ & for both: $160,170,180$ \\
\hline Time (min) & 15 & 15 & 15 & & $5-30$ & $5-30$ \\
\hline
\end{tabular}

\subsubsection{FTIR}

The infrared spectra of the reacted EPDM-samples were recorded by using the attenuated total reflectance infrared technique (ATR/IR) with a Perkin Elmer Spectrum 100. The infrared spectra were recorded with 12 scans at a resolution of 4 $\mathrm{cm}^{-1}$ in the range of $4000-600 \mathrm{~cm}^{-1}$.

\subsubsection{DSC}

DSC was performed on a Perkin Elmer Pyris1 to measure the decomposition temperature of PNO. The samples were heated from $50 \mathrm{oC}$ to $200 \mathrm{oC}$ at a constant rate of $5 \mathrm{oC} / \mathrm{min}$.

\subsubsection{RPA}

A RPA 2000 (Alpha Technologies) was used to measure the crosslinking behaviour of the compounds at $0.833 \mathrm{~Hz}$ and 0.2 degree strain at different temperatures.

\subsection{Results and discussion}

\subsubsection{CBS}

Figure 7.1 shows the ATR/IR spectra of virgin EPDM and CBS-grafted EPDMs. CBS- 1 is formulated according to former works done by Sahakaro et al. ${ }^{1-3}$. A similar result has been achieved in the present study. After reaction, CBS-1 is only partly soluble in a solvent as chloroform and hexane. Compared to virgin EPDM, several additional bands are observed. The band at $1537 \mathrm{~cm}^{-1}$ with strong intensity can be attributed to the $\mathrm{C}=\mathrm{N}$ group in $\mathrm{CBS}$, but it also possibly comes from the $\mathrm{C}=\mathrm{O}$ stretching vibrations of metal-carboxylates due to the formation of the $\mathrm{Zn}$-stearate salt in the presence of stearic acid and $\mathrm{ZnO}$, which are not soluble in acetone and can not be extracted. Two other additional bands at $872 \mathrm{~cm}^{-1}$ and $750 \mathrm{~cm}^{-1}$ are 
relatively weak, but they can be assigned to the aromatic ring in the CBS group, confirming the grafting of CBS onto EPDM.

For CBS-2 and CBS-3, their formulations are simplified compared to CBS-1 as shown in Table 7.1. The amounts of additives, $\mathrm{ZnO}$ and sulphur, are reduced as well to minimize the crosslink formation and to achieve only modification. With much lower amounts of additives, $140^{\circ} \mathrm{C}$ was proved not enough to let the reaction take place; so the temperature was increased to $160^{\circ} \mathrm{C}$. After reaction, the characteristic signals indicating the grafting are very weak and can hardly be observed for CBS-2. Compared to CBS-2, besides CBS and $\mathrm{ZnO}$, a small amount of sulphur was added additionally for CBS-3. CBS-3 is partly in-soluble again indicating partly crosslinking like with CBS-1 after reaction. The ATR/IR spectrum of CBS-3 shows two characteristic bands at $872 \mathrm{~cm}^{-1}$ and $750 \mathrm{~cm}^{-1}$, but not at $1537 \mathrm{~cm}^{-1}$, most probably due to the absence of stearic acid in this formulation. Therefore, grafting takes place for CBS-3, but not for CBS-2. The presence of sulphur in the compounds is necessary to facilitate the grafting reaction, at least at the current reaction conditions: $160^{\circ} \mathrm{C}$ and 15 minutes.

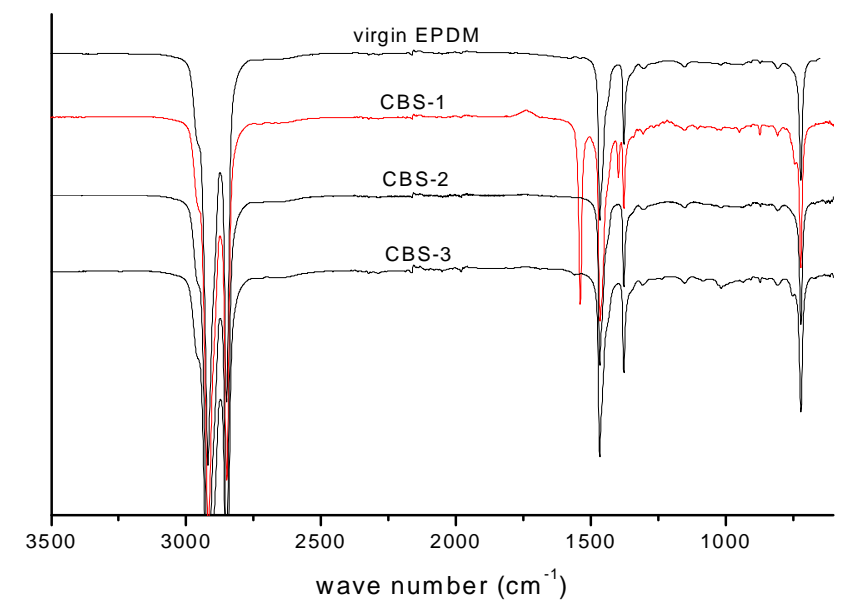

(a)

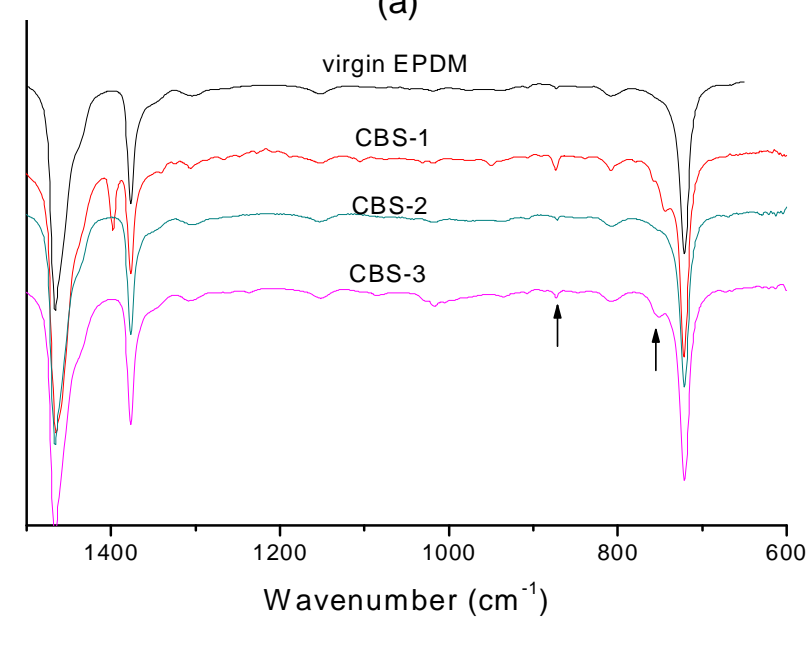

(b)

Figure 7.1 ATR/IR spectra for virgin EPDM and CBS-grafted EPDMs (a): whole range; (b): from $1500 \mathrm{~cm}^{-1}$ to $600 \mathrm{~cm}^{-1}$. 
It can be concluded that grafting of CBS onto EPDM takes place and the proposed reaction is shown in scheme 7.2. However, some crosslinking takes place at the same time and is difficult to be completely avoided. In Sahakaro's work, it was already shown that the pretreated EPDM resulted in a significant improvement of the mechanical properties as compared to virgin EPDM, when it was blended with NR/BR for a tire black sidewall application. The results of Sahakaro with CBSgrafting of EPDM were used as reference for the coming experiments with PNO and CLD.

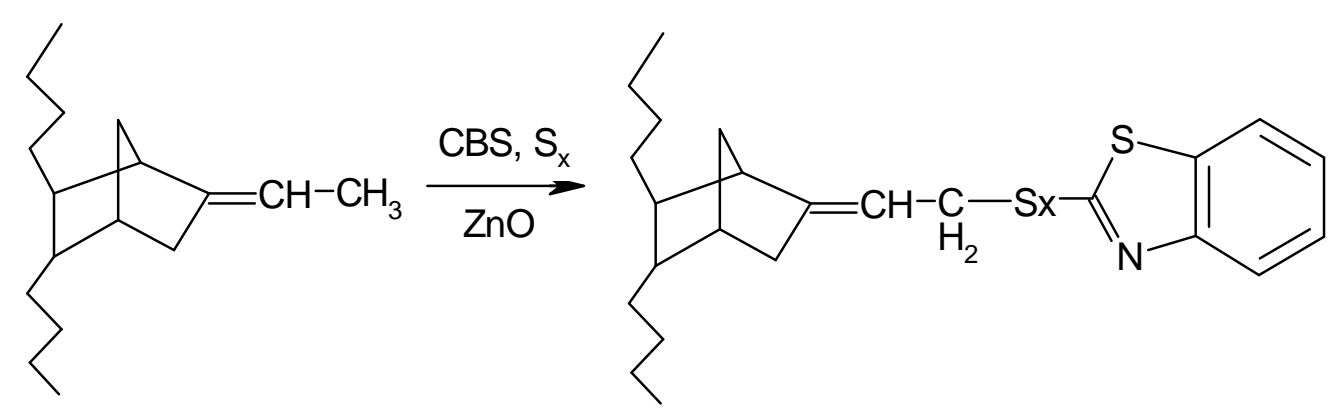

Scheme 7.2 Proposed reaction of EPDM with CBS, sulphur and ZnO.

\subsubsection{PNO}

\subsubsection{DSC}

$\mathrm{PNO}$ and its mixtures as, $\mathrm{PNO} / \mathrm{ZnO}, \mathrm{PNO} / \mathrm{S}, \mathrm{PNO} / \mathrm{ZnO} / \mathrm{S}$, were first tested by DSC to determine the decomposition temperature and the influence of the additive: $\mathrm{ZnO}$ and sulphur, on the thermal behaviour of PNO. The DSC curves are shown in figure 7.2. Pure PNO shows an endothermic peak at about $184^{\circ} \mathrm{C}$ which can be attributed to a decomposition reaction of PNO. The addition of $\mathrm{ZnO}$ to PNO does not change its thermal behaviour. When a small amount of sulphur is mixed with PNO, two overlapping endothermic peaks are observed due to the shift of part of the decomposition-peak to lower temperature at about $170^{\circ} \mathrm{C}$. When both $\mathrm{ZnO}$ and sulphur are added to $\mathrm{PNO}$, an exothermic peak appears at about $135^{\circ} \mathrm{C}$, indicating a combination reaction taking place, which most probably results in the formation of zinc-2-mercaptopyridine $\mathrm{N}$-oxide, as shown in scheme 7.3. A weak decomposition peak then follows at the same temperature of around $170^{\circ} \mathrm{C}$ as the mixture of $\mathrm{PNO} / \mathrm{S}$. Therefore, the presence of only $\mathrm{ZnO}$ has no influence on the decomposition reaction of $\mathrm{PNO}$; only when sulphur is present the decomposition temperature decreases. Based on the DSC results, the modification reactions were then carried out at three different temperatures: $160^{\circ} \mathrm{C}, 170^{\circ} \mathrm{C}$ and $180^{\circ} \mathrm{C}$. 


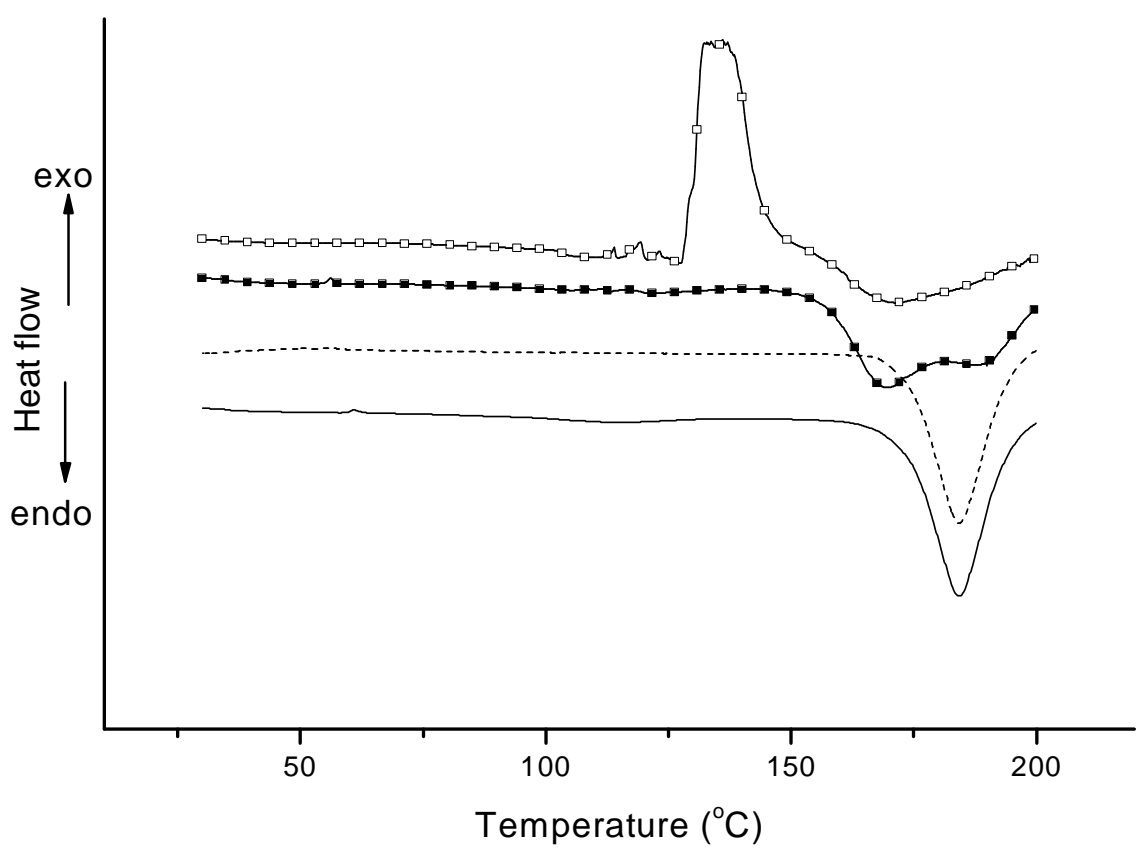

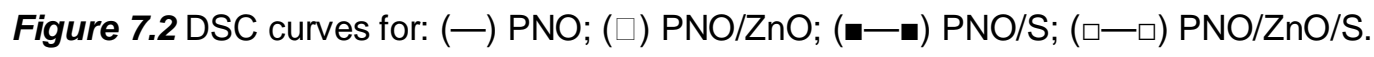<smiles></smiles>

Scheme 7.3 Proposed reaction of PNO with sulphur and ZnO.

\subsubsection{Crosslinking side reactions of EPDM/PNO compounds}

Figure 7.3 shows the RPA cure-curves for different EPDM/PNO compounds: PNO-1, PNO-2 and PNO-3, for modification temperatures varied from $160^{\circ} \mathrm{C}$ to $180^{\circ} \mathrm{C}$. There is almost no increase of the torque value of PNO-1 at $160^{\circ} \mathrm{C}$ and $170^{\circ} \mathrm{C}$. Even at a higher temperature as $180^{\circ} \mathrm{C}$, the torque increases only very slowly. Compared to PNO-1, PNO-2 and PNO-3 have small amounts of sulphur in their formulations and they show a similar trend. Both of them show a limited increase of the torque values under $160^{\circ} \mathrm{C}$. But when the temperature is increased to $170^{\circ} \mathrm{C}$ and $180^{\circ} \mathrm{C}$, the increasing trend becomes more and more pronounced.

It is known that PNO is used as an accelerator for sulphur-cured rubber compounds. The results from the present study also show that it can act as accelerator effectively, especially with small amounts of sulphur combined with a temperature higher than $170^{\circ} \mathrm{C}$. According to the reaction mechanism of accelerators in rubber vulcanisation ${ }^{7}$, the accelerator first interacts with activator and sulphur to form an active sulphurating 
agent. The sulphurating agent then reacts with a rubber hydrocarbon to form a rubber-accelerator intermediate and the intermediate reacts with another rubber molecule to form the real crosslink.

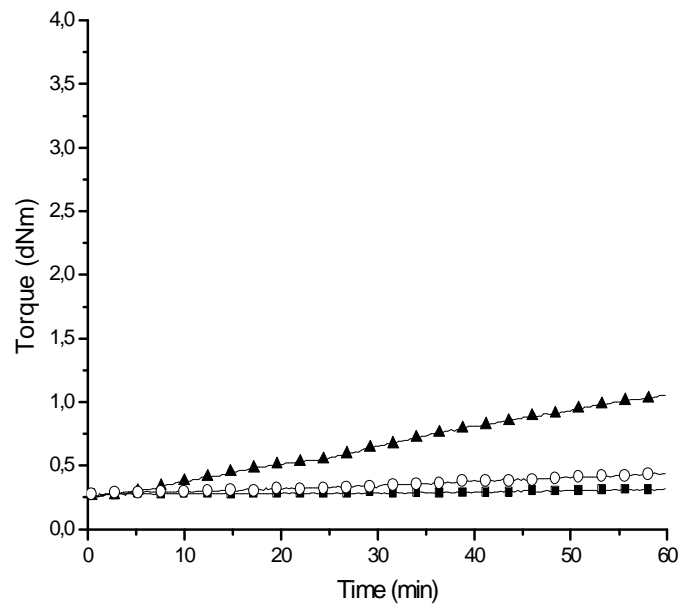

(a) PNO-1

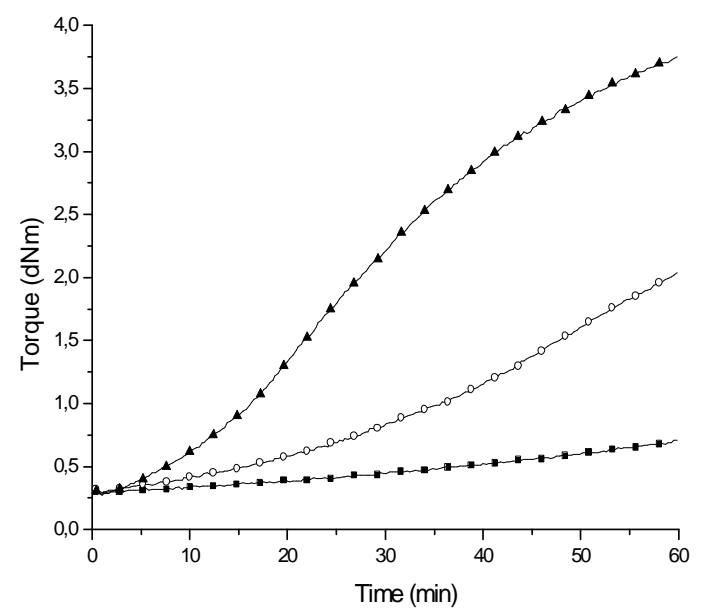

(b) $\mathrm{PNO}-2$

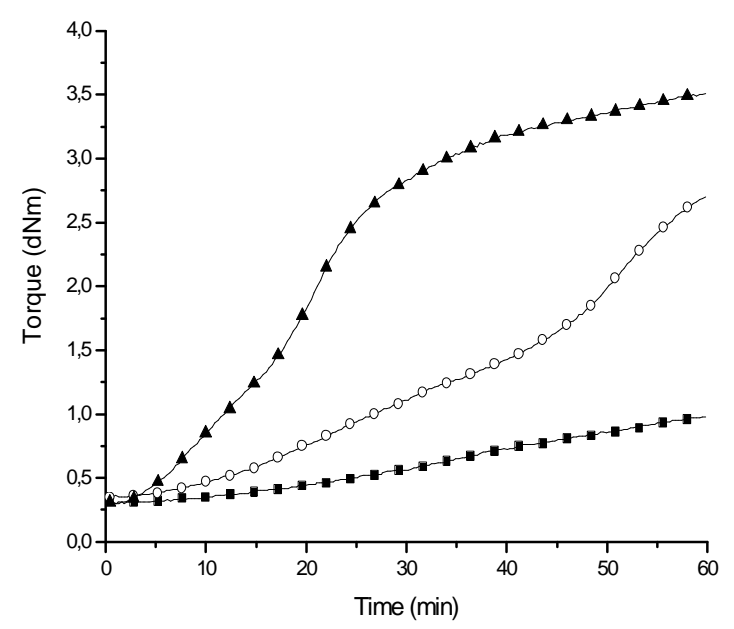

(c) $\mathrm{PNO}-3$

Figure 7.3 RPA curves for (a) PNO-1; (b) PNO-2; (c) PNO-3 at different temperatures: (a-a) $160^{\circ} \mathrm{C} ;(\circ-\circ) 170^{\circ} \mathrm{C} ;(\boldsymbol{\Lambda}-\mathbf{\Lambda}) 180^{\circ} \mathrm{C}$.

In order to achieve grafting and minimize the crosslinking side-reaction, different reaction times were tried, varied from 5 to 30 minutes, according to the various compounds and reaction temperatures. Table 7.3 shows the solubilities of the EPDM/PNO compounds in chloroform after reaction. PNO-1 is still soluble after reaction at $160^{\circ} \mathrm{C}$ and $170^{\circ} \mathrm{C}$, but not at $180^{\circ} \mathrm{C}$. PNO-2 and $\mathrm{PNO}-3$ are still soluble after reaction at $160^{\circ} \mathrm{C}$ for 20 minutes. When the reaction temperature is increased to $170^{\circ} \mathrm{C}$ and $180^{\circ} \mathrm{C}$, they are very apt to become crosslinked even with much shorter reaction times. 
Table 7.3 The solubility of the compounds in chloroform after reaction.

\begin{tabular}{c|cc|ccc|cc}
\hline $\begin{array}{c}\text { Reaction } \\
\text { temperature }\left({ }^{\circ} \mathrm{C}\right)\end{array}$ & \multicolumn{2}{|c|}{160} & \multicolumn{3}{c|}{170} & \multicolumn{2}{c}{180} \\
\hline Reaction time (min) & 30 & 20 & 30 & 20 & 10 & 20 & 5 \\
\hline PNO-1 & $\sqrt{ }$ & - & $\sqrt{ }$ & - & - & $\mathrm{x}$ & - \\
PNO-2 & - & $\sqrt{ }$ & - & $\mathrm{x}$ & $\mathrm{x}$ & - & $\mathrm{x}$ \\
PNO-3 & - & $\sqrt{ }$ & - & $\mathrm{x}$ & $\mathrm{x}$ & - & $\mathrm{x}$ \\
\hline
\end{tabular}

$V=$ soluble; $X$ = insoluble; - = not tested.

\subsubsection{ATR/IR spectra}

All the compounds after reaction under different conditions as shown in Table 7.3 were then characterised by ATR/IR. However, in spite of the different reaction temperatures and times, all the compounds show similar spectra. Figure 7.4 shows the ATR/IR spectra for virgin EPDM, pure PNO and reacted compounds before and after extraction, respectively. Compared to virgin EPDM, the reacted compounds before extraction show some additional bands at $1250 \sim 1200 \mathrm{~cm}^{-1}, 838 \mathrm{~cm}^{-1}$ and $765 \mathrm{~cm}^{-1}$, which can also be found in the spectrum of pure PNO. However, after extraction by acetone, all these bands have disappeared, indicating that PNO has not been grafted onto EPDM. Furthermore, those soluble compounds were also analyzed by ${ }^{1} \mathrm{H}$ NMR, again showing no evidence of grafting.

virgin EPDM

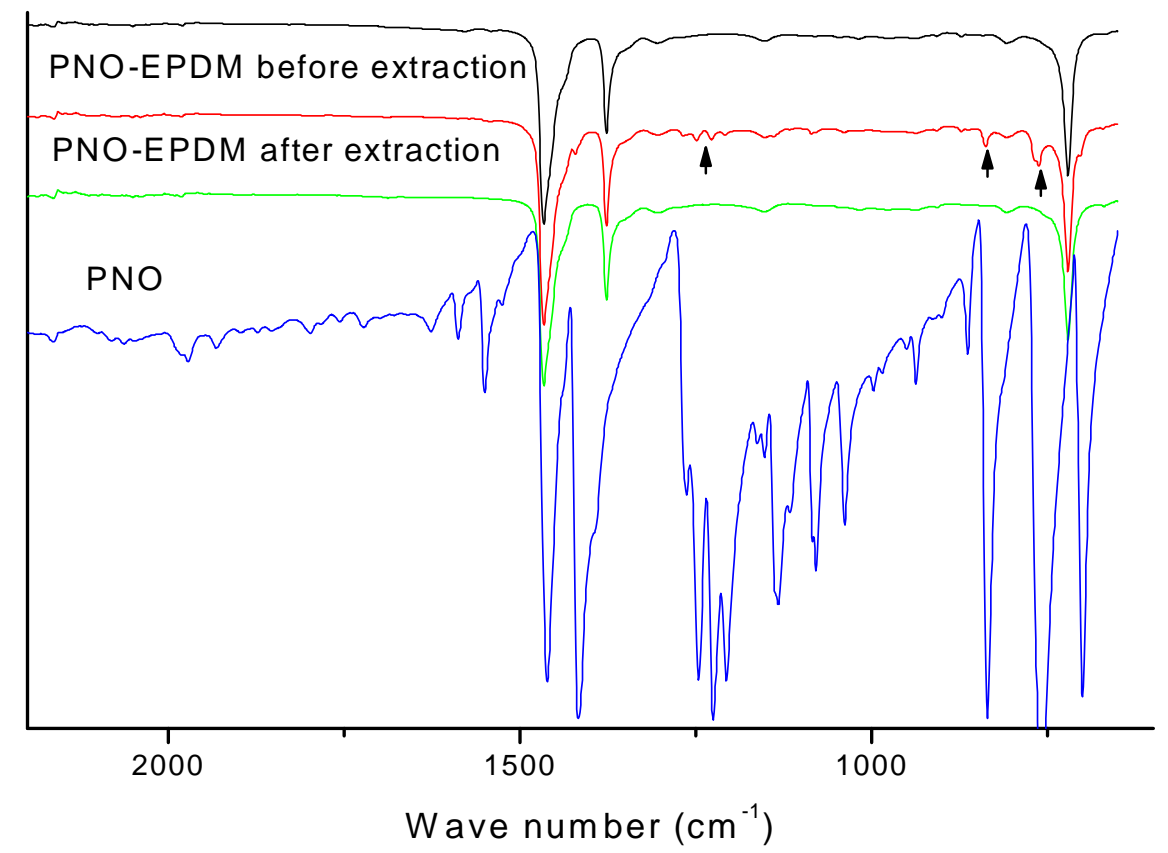

Figure 7.4 ATR/IR spectra for virgin EPDM, pure PNO and reacted EPDM/PNO compounds before extraction and after extraction. 
Above results show that for a temperature of $160^{\circ} \mathrm{C}$, a grafting reaction does not take place at all. But when the temperature is increased to $170^{\circ} \mathrm{C}$ and $180^{\circ} \mathrm{C}$, the compounds are very apt to become crosslinked. Therefore, in the case of PNO, grafting of 2-mercaptopyridine-N-oxide groups may still take place, but apparently the intermediates react immediately with a second rubber-hydrocarbon bond to form crosslinks and to release the pyridine group from the rubber backbone. The reaction is very difficult to be controlled to obtain grafting instead of crosslinking. This compound was therefore not further pursued.

\subsubsection{CLD}

\subsubsection{RPA}

RPA results for CLD-1 and CLD-2 are shown in figure 7.5. At $160^{\circ} \mathrm{C}$, the torque increase of CLD-1 is limited. But when temperature is increased to $170^{\circ} \mathrm{C}$ and $180^{\circ} \mathrm{C}$, a significant increase of the torque value can be observed, which means that the addition of only CLD to EPDM already leads to crosslinking. CLD has been known for long as sulphur donor for rubber vulcanisation. Therefore, extra sulphur is not necessary for this reaction. Considering CLD-2, apparently the extra addition of ZnO does not result in a significant change of the curing curve, indicating that the influence of $\mathrm{ZnO}$ on the reaction is limited or absent.

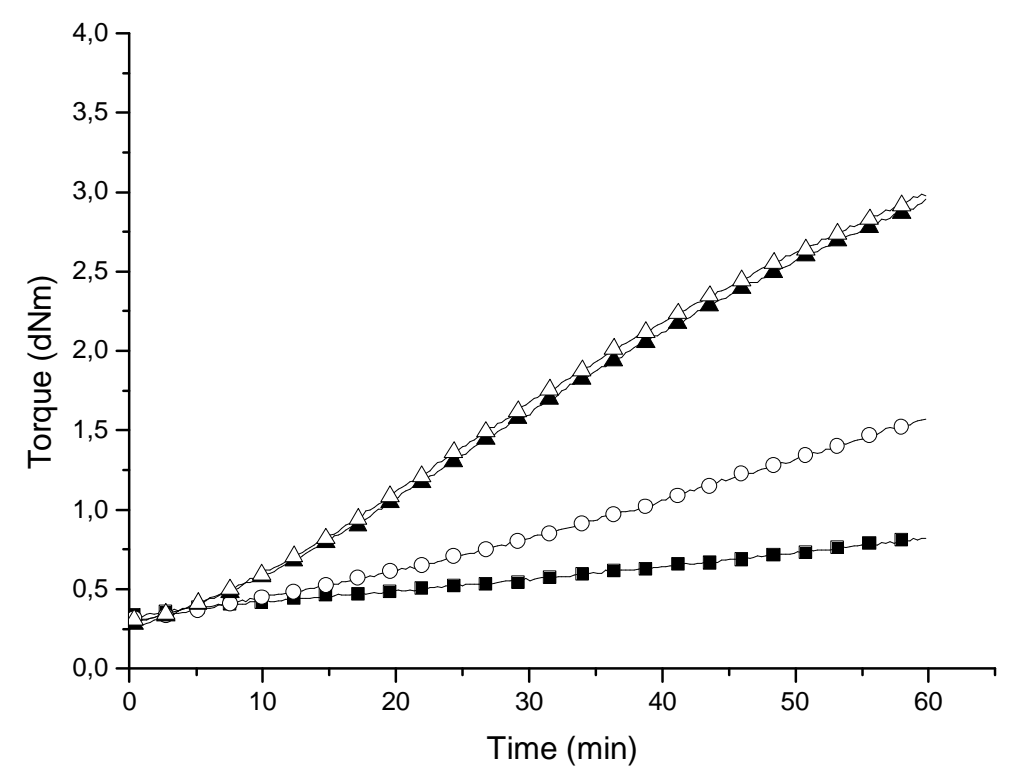

Figure 7.5 RPA curves for CLD-1: (ロ- $160^{\circ} \mathrm{C} ;(\circ-\circ) 170^{\circ} \mathrm{C} ;(\boldsymbol{\Delta}-\boldsymbol{\Delta}) 180^{\circ} \mathrm{C}$ and CLD-2: $(\Delta-\Delta) 180^{\circ} \mathrm{C}$.

\subsubsection{ATR/IR}

Similar to PNO, different temperatures and times were applied for the modification reaction of CLD onto EPDM as well, as shown in Table 7.2. After 
reaction, the compounds were characterised by ATR/IR and the spectra of virgin EPDM and the reacted compounds are shown in figure 7.6. Compared to virgin EPDM, only one additional band can be observed at about $1737 \mathrm{~cm}^{-1}$ for the reacted compounds before extraction. This band can be assigned to the $\mathrm{C}=\mathrm{O}$ bond in CLD, but the intensity of this characterisation band is very weak. The band can hardly be observed anymore after extraction. ${ }^{1} \mathrm{H}$ NMR measurements were tried as well. However, in the NMR spectrum the characteristic signals for CLD overlap with the signals for virgin EPDM, which makes it impossible to obtain evidence of grafting of CLD onto EPDM by this technique.

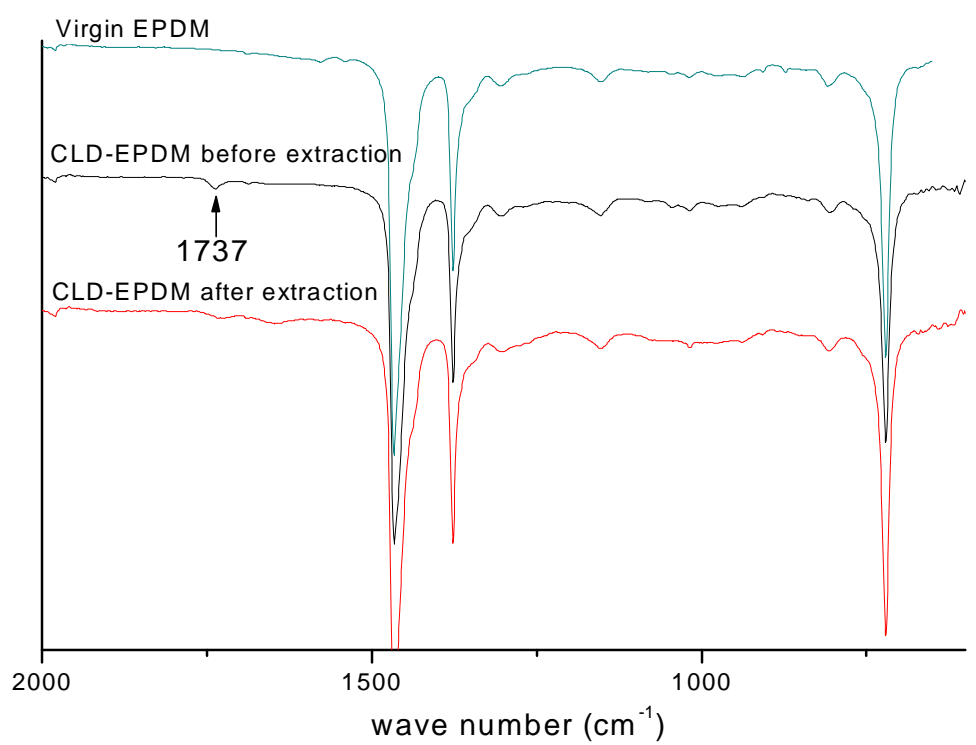

Figure 7.6 ATR/IR spectra for virgin EPDM, reacted EPDM/CLD compounds before and after extraction.

Consequently, there is no evidence that grafting takes place for CLD onto EPDM. The possible reason may be the same as for PNO that the caprolactamrubber intermediate is not stable and reacts very fast with a second rubber molecule to form crosslinks. In addition, the chemicals with di(N-C-S) or di(C-N-S) bonds in their structures are normally very effective sulphur donors. Therefore, for example for CLD, it is also possible that the caprolactam disulphide decomposes to the caprolactam mono-sulphide and sulphur after heating, as shown in scheme 7.4. The sulphur being generated in this way accelerates the crosslinkings of EPDM compounds and restrains grafting from taking place. 


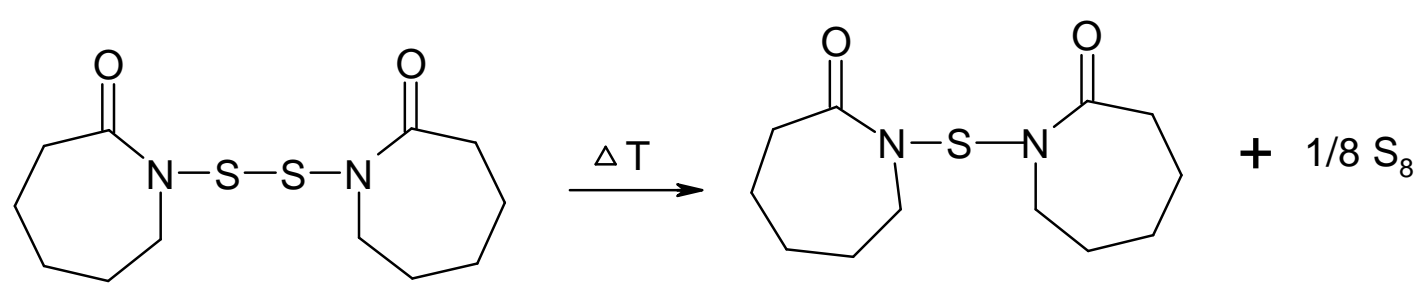

Scheme 7.4 Proposed decomposition reaction of CLD.

\subsection{Conclusions}

The modification by grafting of CBS, PNO and CLD onto EPDM has been studied. From characterisation by ATR/IR, it was observed that grafting of CBS onto EPDM does take place and that a mercaptobenzothiazol-rubber intermediate is generated when EPDM is heated in the presence of CBS, sulfur and $\mathrm{ZnO}$. The compound is partly insoluble in a common solvent after reaction indicating a limited degree of crosslinking or gelation by the grafting reaction. Various compositions, reaction temperatures and reaction times have been tried for a grafting reaction of PNO and CLD onto EPDM. However, both are very sensitive to become crosslinked during the reaction. No evidence from $A T R / I R$ and $\mathrm{H}^{1}$ NMR shows that PNO and CLD could be grafted onto EPDM under the present conditions.

\section{REFERENCES}

1. Sahakaro, K.; Naskar, N.; Datta, R. N.; Noordermeer, J. W. M., J. Appl. Polym. Sci., 103, 2538 (2007).

2. Sahakaro, K.; Talma, A. G.; Datta, R. N.; Noordermeer, J. W. M., J. Appl. Polym. Sci., 103, 2547 (2007).

3. Sahakaro, K.; Datta, R. N.; Baaij, J.; Noordermeer, J. W. M., J. Appl. Polym. Sci., 103, 2555 (2007).

4. Davis, L. H.; Sullivan, A. B.; Coran, A. Y., Rubber Chem. Technol., 60, 125 (1987).

5. Rostek, C. J.; Lin, H. J.; Sikora, D. J.; Katritzy, A. R.; Kuzmierkiewicz, W.; Shobana, N., Rubber Chem. Technol., 69, 180 (1996).

6. Datta, R. N.; Huntink, N. M.; Nieuwenhuis, P.; Talma, A., WO patent 0116227 (A1), to: Flexsys B.V., priority: August 31, 1999.

7. Borros, S.; Agullo, N., Kautsch. Gummi Kunstst., 53, 131 (2000). 


\title{
Chapter 8
}

\section{Modification of EPDM with Alkylphenol Polysulphide for Use in Tyre Sidewalls: Part I. Mechanical Properties}

\begin{abstract}
Alkylphenol polysulphide (APPS) has been used to modify EPDM in order to solve the cure incompatibility and heterogeneous filler distribution of NR/BR/EPDM blends for tyre sidewall applications. In this study, the physical properties of the NR/BR/APPS-EPDM blends are tested in comparison with an NR/BR/EPDM blend and a conventional NR/BR tyre sidewall compound. It is demonstrated that the application of APPS-grafted EPDM leads to a significant improvement of the tensile properties, tear strength and fatigue properties compared to virgin EPDM. These physical properties of the NR/BR/APPS-EPDM blends are equivalent or even superior to those of conventional NR/BR tyre sidewall compounds. The dynamic viscoelastic properties of the NR/BR/APPS-EPDM blends are not quite comparable with the conventional NR/BR sidewall blend, but still greatly improved compared to using virgin EPDM.
\end{abstract}




\subsection{Introduction}

The main problems associated with the generally poor properties of EPDM/high-diene rubber blends are the difference in molar concentrations of carboncarbon double bonds in each of the elastomers, resulting in differences in polarity, number of allylic sites for sulphur vulcanisation, and reactivity of the crosslink sites ${ }^{1}$. Curatives, which are commonly polar molecules, generally diffuse preferentially into the higher unsaturated elastomers i.e. the diene phases, resulting in differences in concentration of reactants and hence uneven crosslink distribution ${ }^{2,3}$. Carbon black also prefers to migrate into the higher unsaturated polymers causing a heterogeneous filler distribution in the blends ${ }^{4,5}$. Several approaches have been proposed to overcome these problems and to improve the properties of EPDM/highdiene rubber blends. Generally, these attempts have sought to increase the cure rate of EPDM, either by using curatives that have an increased reactivity towards EPDM, or by means of modifying the EPDM to make it more reactive towards curing ${ }^{6-12}$ and more attractive to fillers.

Cook $^{13}$ made a similar attempt by a different procedure called "reactive mixing". In his work, three commercially available sulphur donors, bis-alkylphenoldisulphide (BAPD), dithiodicaprolactam (DTDC) and dithiodimorpholine (DTDM) were used to modify EPDM by mixing at elevated temperatures in an internal mixer as part of a normal masterbatch mixing cycle. The modification is believed to be similar in nature to what was described by Hopper $^{14}$, in which a certain functionality: alkylphenolmonosulphide, caprolactam or morpholine coming from the sulphur donor was attached to the EPDM polymer molecules via a sulphur linkage. During vulcanisation, all of these groups could act as leaving groups, to be substituted by MBT which is known as a vulcanisation intermediate. In this way a crosslink precursor site on the EPDM polymer chain was formed.

In the present work alkylphenol polysulphide (APPS) has been selected as modification agent for EPDM. Alkylphenol sulphides were introduced in the 1940s as sulphur donors for synthetic elastomers. They are particularly recommended for Butyl- and Halobutyl rubber-based applications ${ }^{15-18}$. There are several benefits by using alkylphenol sulphides as curing agents: they are nitrosamine free, have no blooming problems, provide better heat resistance, improve dynamic fatigue properties, enhance adhesion and may act as tackifier. Moreover, due to the phenol group in their structure, they are believed to have a good affinity with carbon black, which may improve the carbon black distribution after EPDM modification and consequently the mechanical properties. 


\subsection{Experimental}

\subsubsection{Materials}

Poly (para-tertiary-butyl-phenol-disulphide) (APPS) or Vultac TB7 (Arkema, France) was used as modification agent. The chemical structure is shown in scheme 8.1.

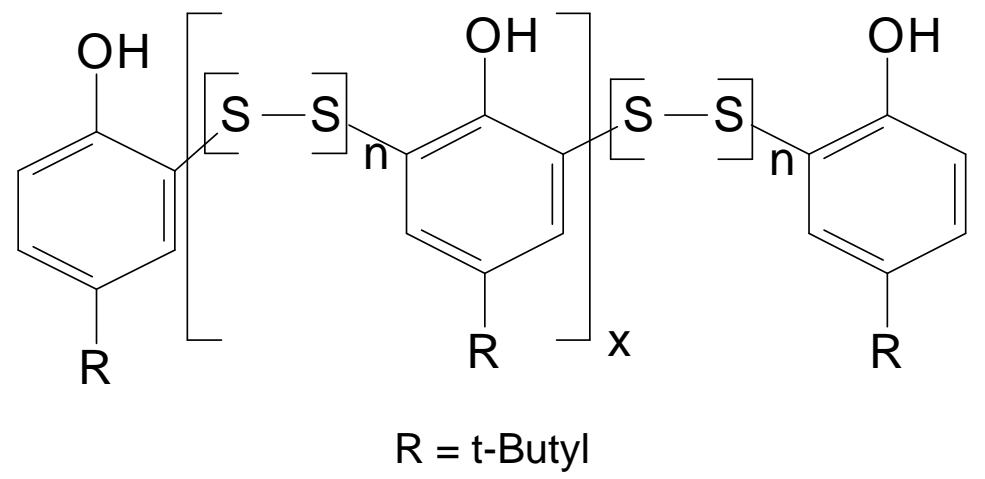

Scheme 8.1. Chemical structure of APPS

The elastomers selected for the blends were Natural Rubber (SIR20, Standard Indonesian Rubber), Butadiene Rubber (Kosyn KBR01, Korea Kumho Petrochemical Co., Ltd), EPDM rubber containing 4.5wt\% ethylidene norbornene (ENB) as third monomer and 70wt\% of ethylene (Keltan 5508, DSM Elastomers B.V., the Netherlands).

The other compounding ingredients used were extra pure grade zinc oxide (Merck, Germany), finely divided sulphur (Merck, Germany), 95\% pure stearic acid (Aldrich, Germany), poly (2,2,4-trimethyl-1,2-dihydroquinoline) or TMQ (Flexsys B.V., the Netherlands) and N-cyclohexyl-2-benzothiazolesulphenamide (CBS) or Santocure ${ }^{\circledR}$ (Flexsys B.V., the Netherlands). High abrasion furnace black (HAF-N330, Cabot Corporation) and naphthenic oil Sunthene 4240 (Sun Petroleum Products Co., Ltd.) were used in the case of filled blends.

\subsubsection{Grafting of APPS onto EPDM}

Grafting of APPS onto EPDM was done in a Brabender PL2000 laboratory internal mixer with a chamber volume of $50 \mathrm{~cm}^{3}$, using a rotor speed of $80 \mathrm{rpm}$, a fill factor of 0.8 and an initial temperature of $165^{\circ} \mathrm{C}$. The amount of APPS was varied from $2 w t \%$ to $10 w t \%$ relative to the EPDM.The EPDM was first masticated for 1 minute, then APPS was added and the mixing was continued for another 10 minutes. The final compound temperature as recorded before dumping from the mixer was in the range of $183 \pm 2^{\circ} \mathrm{C}$. 


\subsubsection{Preparation and vulcanisation of the compounds}

The overall formulations of the compounds are given in Table 8.1. The blend compounds were prepared in a Brabender Plasticorder 350 S mixer having a mixing chamber bolume of $370 \mathrm{~cm}^{3}$. The mixer was operated at a rotor speed of $80 \mathrm{rpm}$, fill factor of 0.7 and an initial temperature of $50^{\circ} \mathrm{C}$. NR was first masticated for 2 minutes, then BR and EPDM or APPS-EPDM were added and mixed for another 1 minute. $\mathrm{ZnO}$, stearic acid, and $\mathrm{TMQ}$ were subsequently added and the mixing was continued for again 2 minutes. The final compound temperature before dumping from the mixer was in the range of $110-115^{\circ} \mathrm{C}$. CBS and sulphur were added to the compounds on a two-roll mill.

In the case of carbon black filled compounds, the same conditions were applied. NR was first masticated for 2 minutes, then BR and EPDM or APPS-EPDM were added and mixed for 1 minute. After that, HAF black, oil and other additives were added. CBS and sulphur were again added to the masterbatch on the two-roll mill.

Table 8.1. Formulation of NR/BR/EPDM or grafted-EPDM compounds

\begin{tabular}{l|cccc}
\hline Component & Ref-0 & Ref-1 & APPS-X* & APPS-straight \\
\hline NR SIR 20 & 50 & 35 & 35 & 35 \\
BR Kosyn KBR01 & 50 & 35 & 35 & 35 \\
EPDM Keltan 5508 & - & 30 & - & 30 \\
APPS-EPDM & - & - & $30+0.3^{*} X$ & - \\
APPS & - & - & - & 2.3 \\
Zinc Oxide & 4 & 4 & 4 & 4 \\
Stearic acid & 2 & 2 & 2 & 2 \\
TMQ & 1 & 1 & 1 & 1 \\
6PPD & 2 & - & - & - \\
CBS & 1.98 & 1.98 & 1.98 & 1.98 \\
Sulphur & 2.5 & 2.5 & 2.5 & 2.5 \\
\hline CB HAF N330 & 50 & 50 & 50 & 50 \\
Oil & 10 & 10 & 10 & 10 \\
\hline *X Amount of APPS in wo used for modification of 100mt\% EPDM
\end{tabular}

${ }^{*} X=$ Amount of APPS in wt $\%$ used for modification of $100 w t \%$ EPDM

The resulting compounds were tested for their cure characteristics using a RPA 2000 cure meter (Alpha Technologies) according to ISO 6502. The optimum cure time $\left(t_{c}, 90\right)$ of the fully compounded blends was determined as the time needed 
to reach $90 \%$ of the maximum torque difference in the RPA 2000 at $140^{\circ} \mathrm{C}, 0.833 \mathrm{~Hz}$ and 0.2 degree strain. The blends were then vulcanised for this optimum cure time $\mathrm{t}_{\mathrm{c}, 90}$ in a Wickert WLP1600 laboratory compression press at $140^{\circ} \mathrm{C}$ and $100 \mathrm{bar}$.

\subsubsection{Tensile and trouser tear tests}

Type 2 dumb-bell test pieces were die-cut from the compression-molded sheet sand tensile tests were carried out according to ISO 37, with a Zwick tensile tester Model Z 1.0/TH1S at a constant crosshead speed of $500 \mathrm{~mm} / \mathrm{min}$.

Trouser test specimens of $100 \mathrm{~mm} \times 7.5 \mathrm{~mm}$ with a cut of depth $40 \mathrm{~mm}$ were die-cut and the test was performed according to ISO 34, Method A with the Zwick tensile tester at a constant crosshead speed of $100 \mathrm{~mm} / \mathrm{min}$.

\subsubsection{Flex fatigue to failure tests}

Fatigue-to-failure tests were carried out on a Monsanto fatigue-to-failure tester at constant strain energy. To determine the strain energy, a cured fatigue specimen was first conditioned by cycling it 30 times to $140 \%$ extension. Using ASTM procedure D-412, a stress-strain curve was then generated from which the force per unit area from $10 \%$ to $140 \%$ extension in $10 \%$ increments was determined. From these data, three extension ratios and corresponding strain energies were selected for each sample.

Dumb-bell test specimens were subjected to stress-strain cycles at the preselected extension ratios and the number of cycles required to cause failure, as indicated by complete rupture of the test specimen, was recorded. 12 Test specimens of each sample were tested and the median value of the number of cycles required to cause failure was recorded. For each sample, three fatigue life values were measured for the three pre-determined extension ratios, respectively.

The fatigue life at a reference strain energy was then interpolated from the three respective fatigue lives per sample, using the following equation ${ }^{19}$ :

$$
\ln (\text { FatigueLife })=A+B[\ln (\text { StrainEnergy })]
$$

The reference strain energy was $1.2 \mathrm{MPa}$ for gum blends and $1.8 \mathrm{MPa}$ for the HAF-filled blends.

\subsubsection{Frequency sweep measurements}

Frequency sweep measurements of the unvulcanised and vulcanised compounds were performed in the RPA 2000. The frequency sweeps of 
unvulcanised compounds were done at $100^{\circ} \mathrm{C}$ and $14 \%$ strain and of vulcanised compounds at $60^{\circ} \mathrm{C}$ and $3.5 \%$ strain. For the case of vulcanised compounds, the specimens were first vulcanised at $t_{c, 90}$ at $140^{\circ} \mathrm{C}$, followed by a cooling step to $60^{\circ} \mathrm{C}$. The frequency, in both cases, varied from $0.01 \mathrm{~Hz}$ to $30 \mathrm{~Hz}$.

\subsubsection{Accelerated heat-aging experiments}

Accelerated heat-aging experiments were carried out according to ISO 188, method $B$, on dumb-bell shaped specimens. The specimens were kept in a nonaerated oven at $70 \circ \mathrm{C}$ during time periods ranging from 1 till 30 days.

\subsection{Results and Discussion}

\subsubsection{Grafting of EPDM with APPS in an internal mixer}

Grafting of EPDM with different APPS-amounts was done in the smaller Brabender PL2000 internal mixer. Figure 8.1 shows the representative torque and temperature curves during mixing. After the addition of APPS, the torque decreases and then rises to its previous value. The softening temperature for APPS is about $106^{\circ} \mathrm{C}$. Therefore, when added to the hot compounds at $160^{\circ} \mathrm{C}$, the viscosity of the mixing compounds decreases. Higher APPS amounts make the compounds take more time to return to their previous torques. With mixing going on, the temperature stays stable at about $183^{\circ} \mathrm{C}$ and the torque values stay more or less at a constant value.

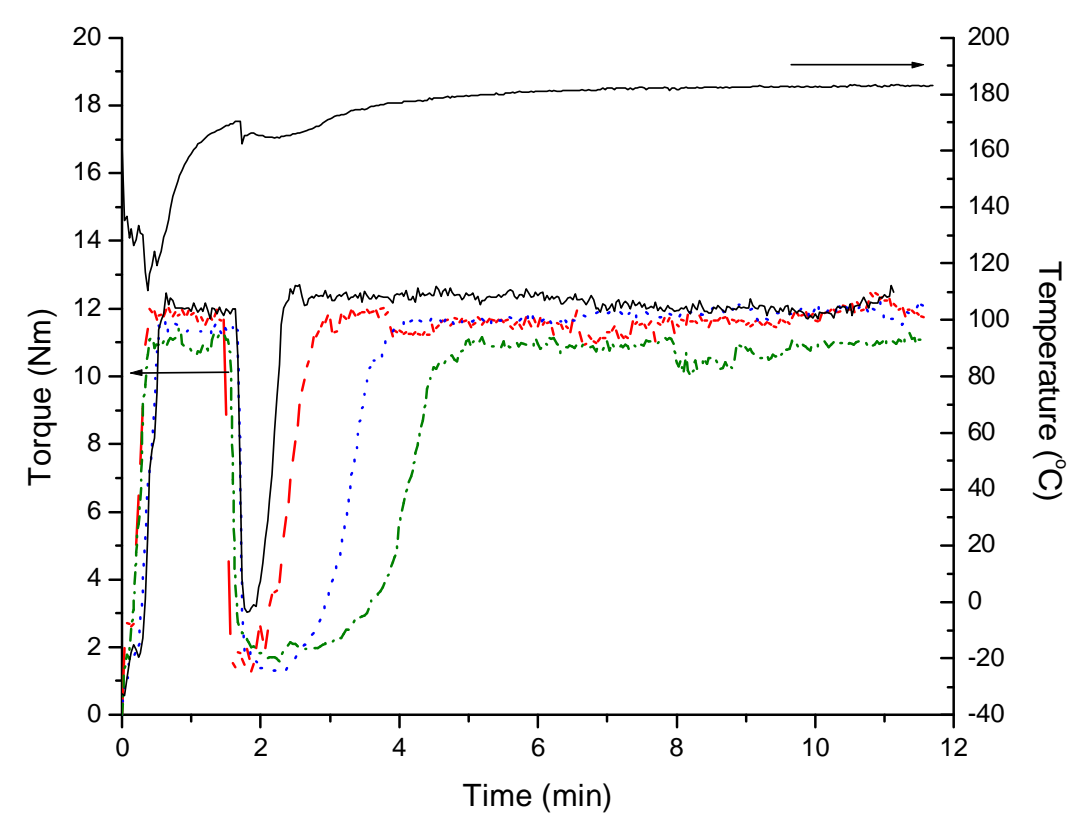

Figure 8.1 Torque and temperature curves during mixing of EPDM with different amounts of APPS: (-) 3wt\% APPS; (---) 5wt\% APPS; (...) 7wt\% APPS; (-.-) 10wt\% APPS. 


\subsubsection{APPS modified-EPDM in NR/BR/EPDM blends}

\subsubsection{Curing characteristics}

Figure 8.2 shows the curing curves for HAF-filled NR/BR and NR/BR/(APPS)EPDM blends after mixing according to the recipes in Table 8.1. A clear reversion can be observed for Ref-0 as opposed to the EPDM containing blends. The addition of unmodified EPDM to the NR/BR blend: Ref-1, increases both scorch and cure time. On the other hand, with APPS-grafted EPDM, the scorch time decreases and becomes even shorter than that of the straight NR/BR blend: Ref- 0 . With respect to the maximum torque levels, the blends with APPS-grafted EPDM show higher maximum torques than the NR/BR and NR/BR/EPDM blends. The maximum torque value further increases with higher APPS amounts. As alkylphenol polysulphide contains about $30 w t \%$ sulphur, the consequence of the APPS-grafting is that more sulphur is introduced into the blends with APPS-grafted EPDM, and therefore a higher state of cure is obtained.

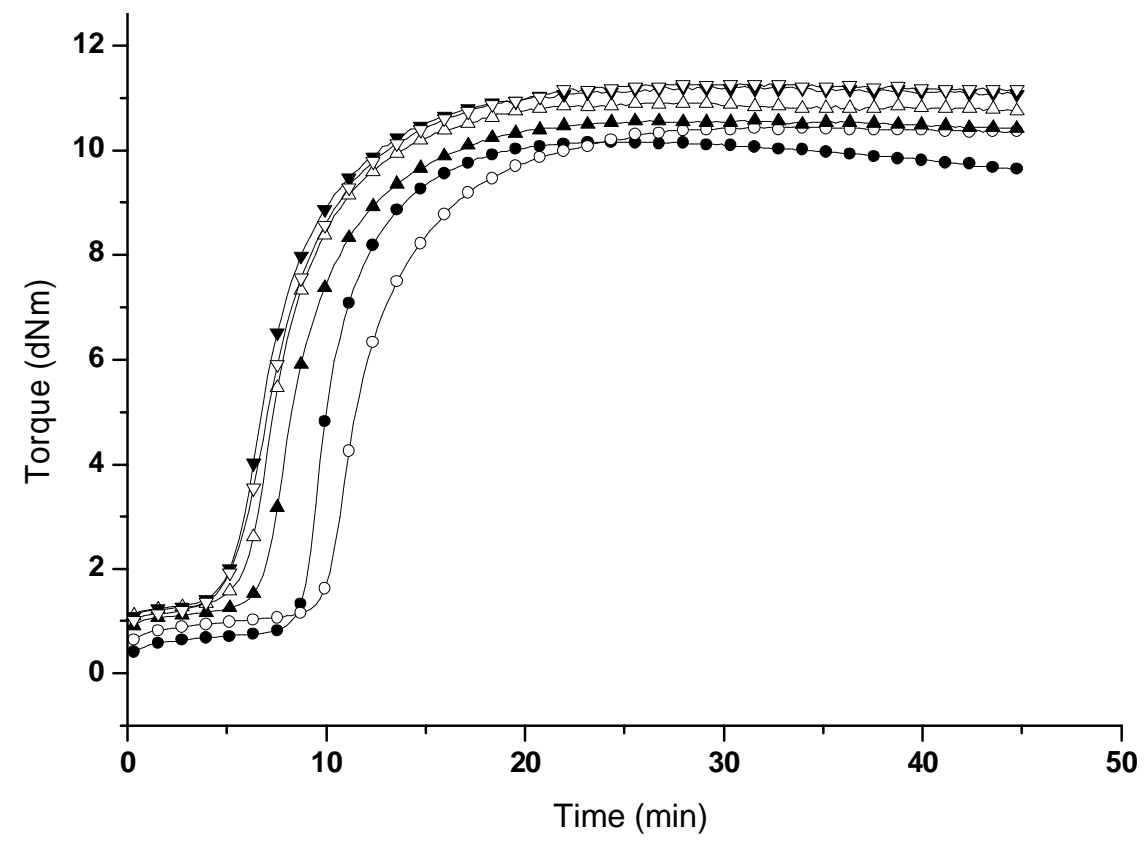

Figure 8.2 RPA curing curves for HAF-filled NR/BR and NR/BR/(APPS-)EPDM blends:

$(\bullet)$ : Ref-0; (०): Ref-1; ( $\mathbf{\Delta})$ : APPS-3; ( $)$ : APPS-5; ( $\mathbf{\nabla})$ : APPS-7; ( $)$ : APPS-10.

For gum NR/BR and NR/BR/(APPS-)EPDM blends, similar results are found for their curing behaviours, as shown in figure 8.3. However, the scorch time decreases more significantly with the addition of APPS-grafted EPDM. The grafted APPS-group may react with CBS, accelerate the decomposition of CBS and speed up the curing rate. 


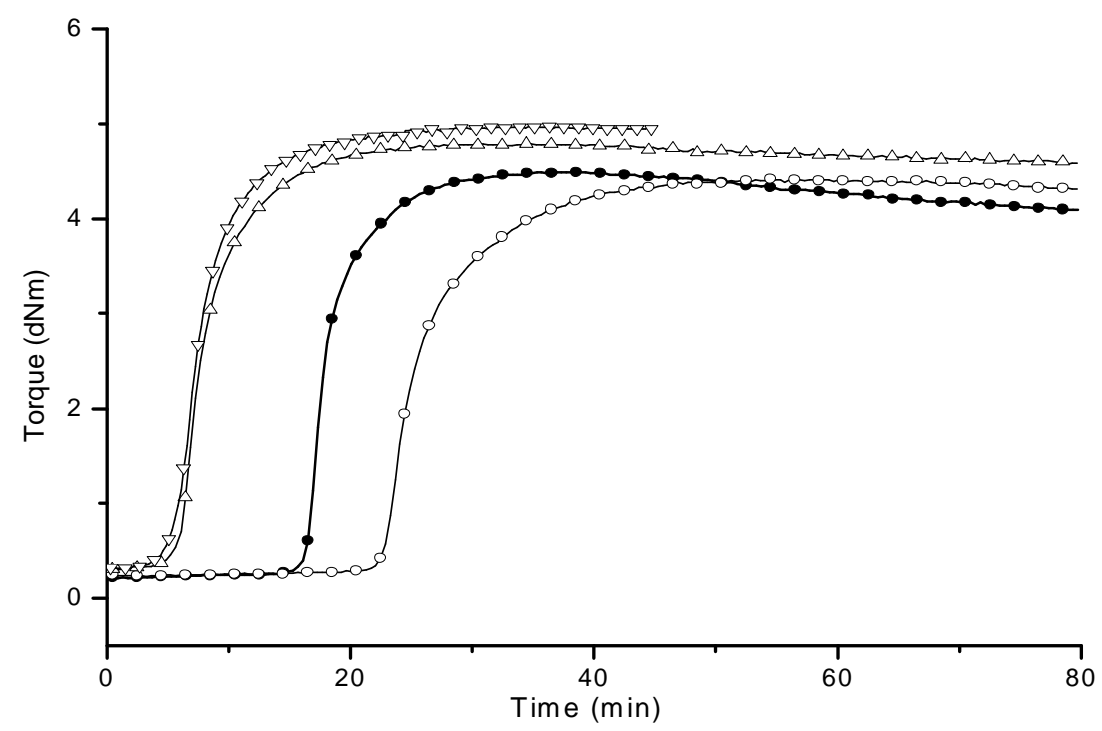

Figure 8.3 RPA curing curves for gum NR/BR and NR/BR/(APPS-)EPDM blends:

$(\bullet)$ : Ref-0; (०): Ref-1; (口): APPS-5; (口): APPS-10.

\subsubsection{Tensile Properties}

Figures 8.4 and 8.5 show the stress-strain curves for gum and HAF-filled blends, respectively. Both gum- and HAF-filled-Ref1 show much poorer tensile properties than the NR/BR blend most probably due to some commonly existing problems in such kind of blends, as: viscosity mismatch, thermodynamic incompatibility, cure incompatibility and inhomogeneous filler distribution, as will be further covered in Chapter 9. With APPS-grafted EPDM, the tensile properties improve significantly compared to the blend with virgin EPDM and are comparable with the NR/BR blend. The characteristics data of the tensile properties are summarized in figures 8.6, 8.7 and 8.8.

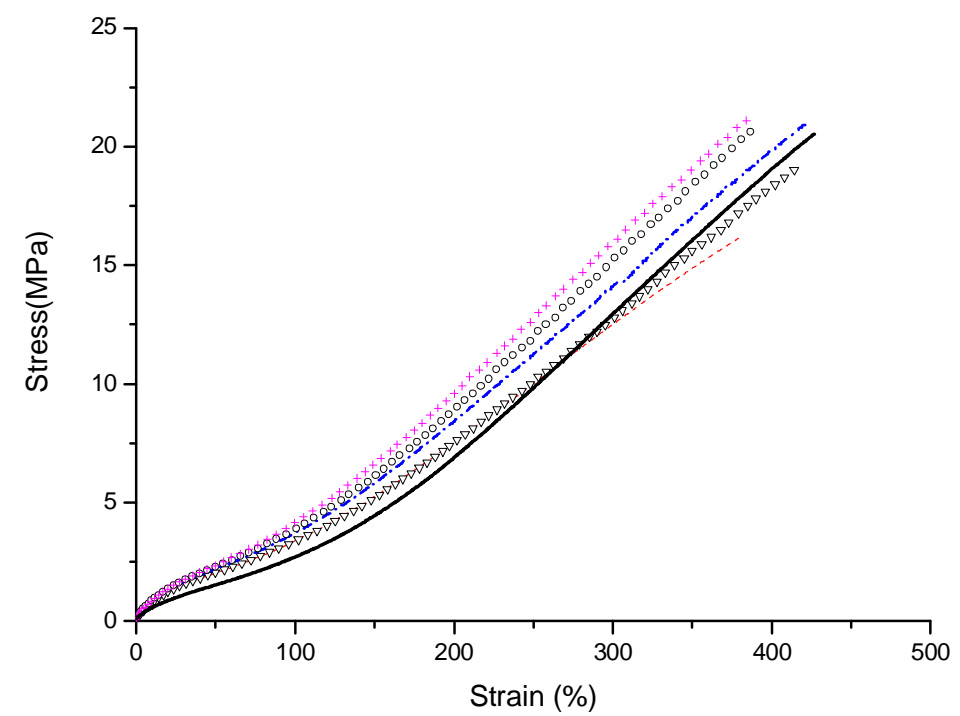

Figure 8.4 Tensile curves for HAF-filled NR/BR and NR/BR/(APPS-)EPDM blends: $(-)$ : Ref-0; (...): Ref-1; ( o): APPS-3; (-.-): APPS-5; (॰o): APPS-7; (+++): APPS-10. 


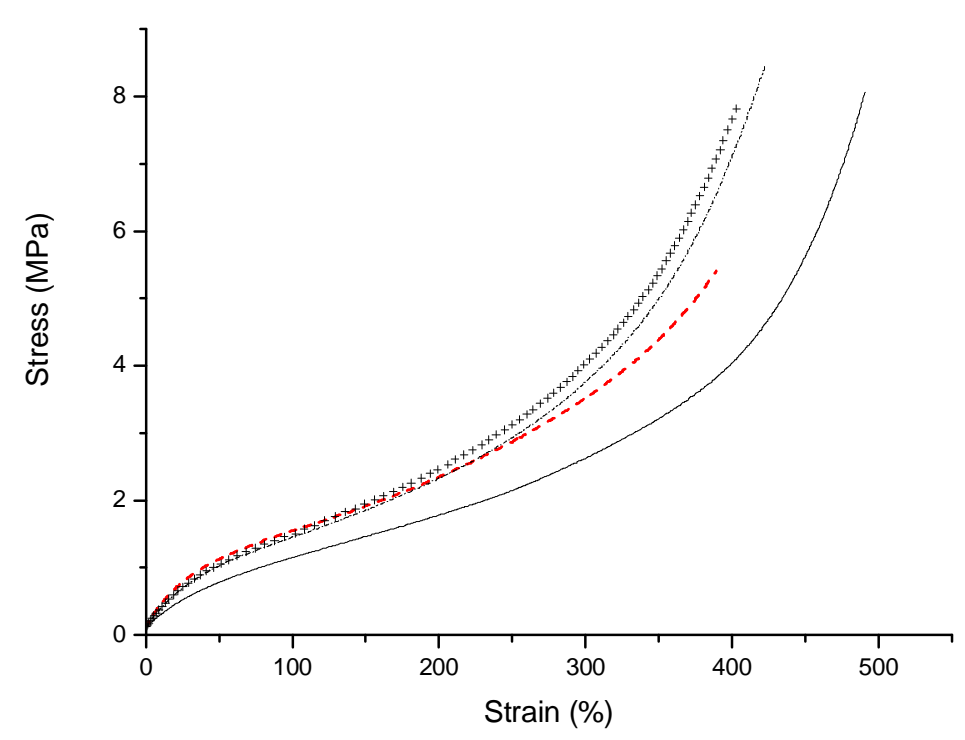

Figure 8.5 Tensile curves for gum NR/BR and NR/BR/(APPS-)EPDM blends:

$(-)$ : Ref-0; (..): Ref-1; (-.-): APPS-5; (+++): APPS-10.

Figure 8.6 shows the tensile moduli at $100 \%$ and $300 \%$ strain for both the HAF-filled and gum blends. For the filled blends, Ref-1 has a higher $100 \%$-modulus but lower 300\%-modulus compared tot Ref-0. When APPS-grafted EPDM is applied, the moduli increase continuously with higher APPS-amounts. For the case of the gum blends, all EPDM-containing blends exhibit higher moduli, at both $100 \%$ and $300 \%$ strain, than the gum NR/BR blend. After EPDM-grafting by APPS, the moduli increase as well. These results are consistent with the maximum torque values from the curing cures. As already discussed before, the extra sulphur content from the APPS and a higher resultant crosslink density may cause the contribution to the higher modulus with APPS-grafted EPDM.

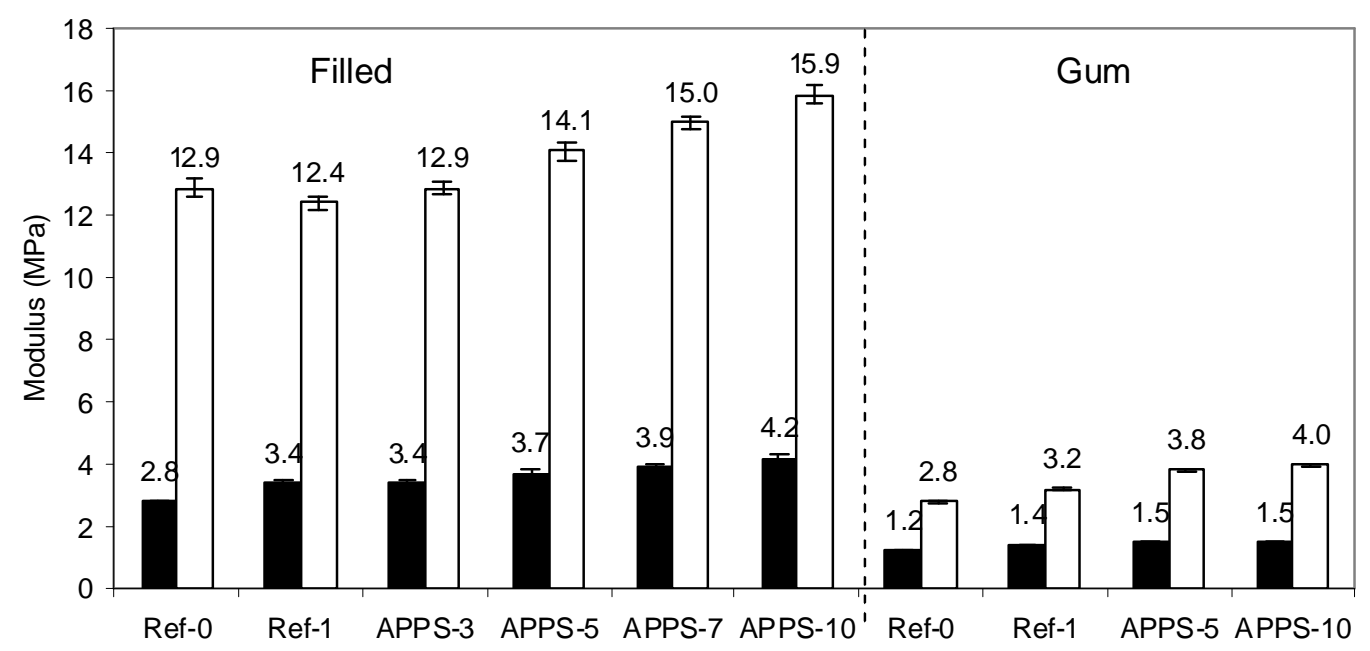

Figure 8.6 Modulus at $100 \%$ and $300 \%$ strain for NR/BR and NR/BR/(APPS-)EPDM blends: ( -) at $100 \%$ strain; () at $300 \%$ strain. 
The tensile strengths of the HAF-filled and gum blends are shown in figure 8.7. Ref- 1 has much lower tensile strength than Ref-0 in both the filled and gum cases. When APPS-grafted EPDM is applied, a significant improvement is obtained compared to the blend with virgin EPDM. The tensile strength with grafted EPDM is even higher than for the NR/BR blend for both filled and gum blends. However, between the different APPS-amounts grafted onto EPDM, there are no significant differences. 3wt\% APPS-grafted EPDM already shows a large improvement. With APPS-amounts higher than $5 \mathrm{wt} \%$, no further improvement of the tensile strength can be observed anymore. The significant improvement of the tensile strength may be attributed to a higher crosslinking density due to extra sulphur but also to an improved solubility of curatives and better carbon black distribution in the EPDM phase after EPDM modification: see Chapter 9.

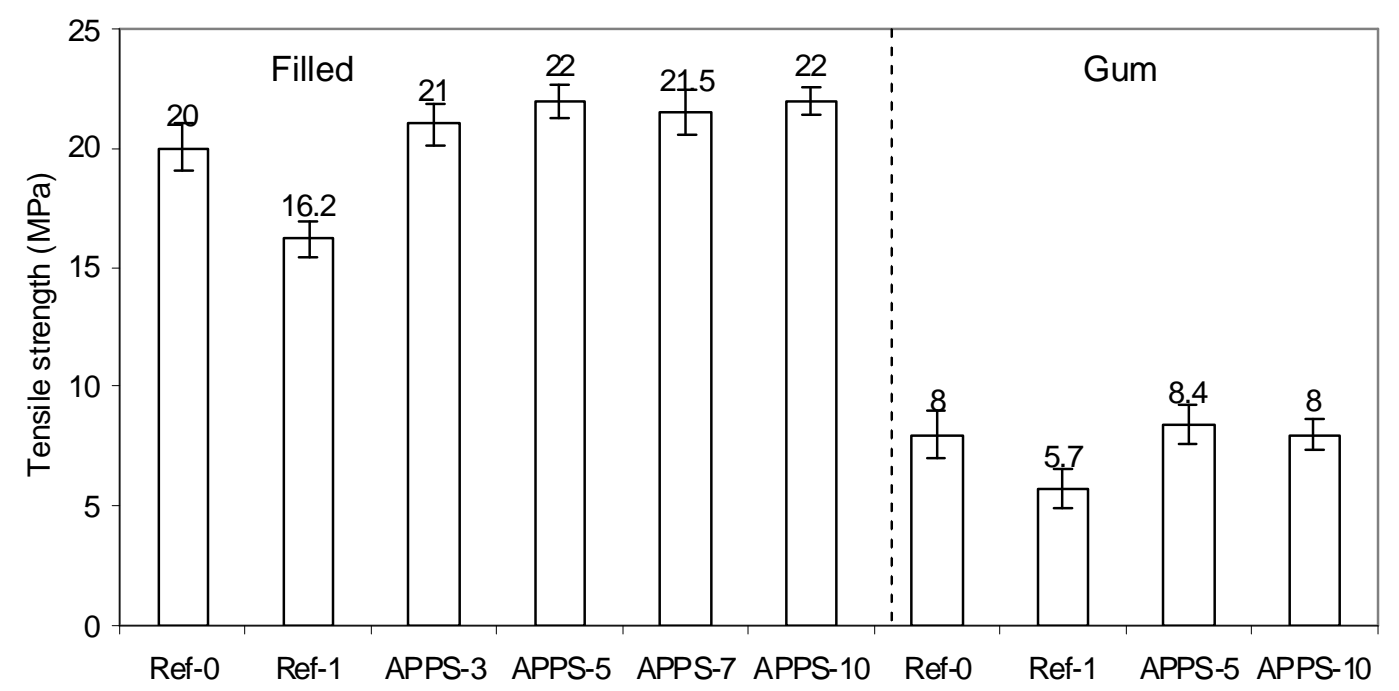

Figure 8.7Tensile strength for NR/BR and NR/BR/(APPS-)EPDM blends.

Figure 8.8 shows the elongation at break for the HAF-filled and gum blends. Both types of blends show a similar trend. From Ref- 0 to Ref- 1 , a clear decrease can be seen like in the tensile strength. Compared to Ref-1, an improvement of the elongation can be observed again when 3wt\% APPS-grafted EPDM is applied. With increasing APPS-amounts, the elongation at break subsequently decreases when the APPS-amount is higher than $5 w t \%$. It is clear that with higher APPS-amounts, modulus increases while elongation at break decreases and the tensile strength remains more or less the same. 


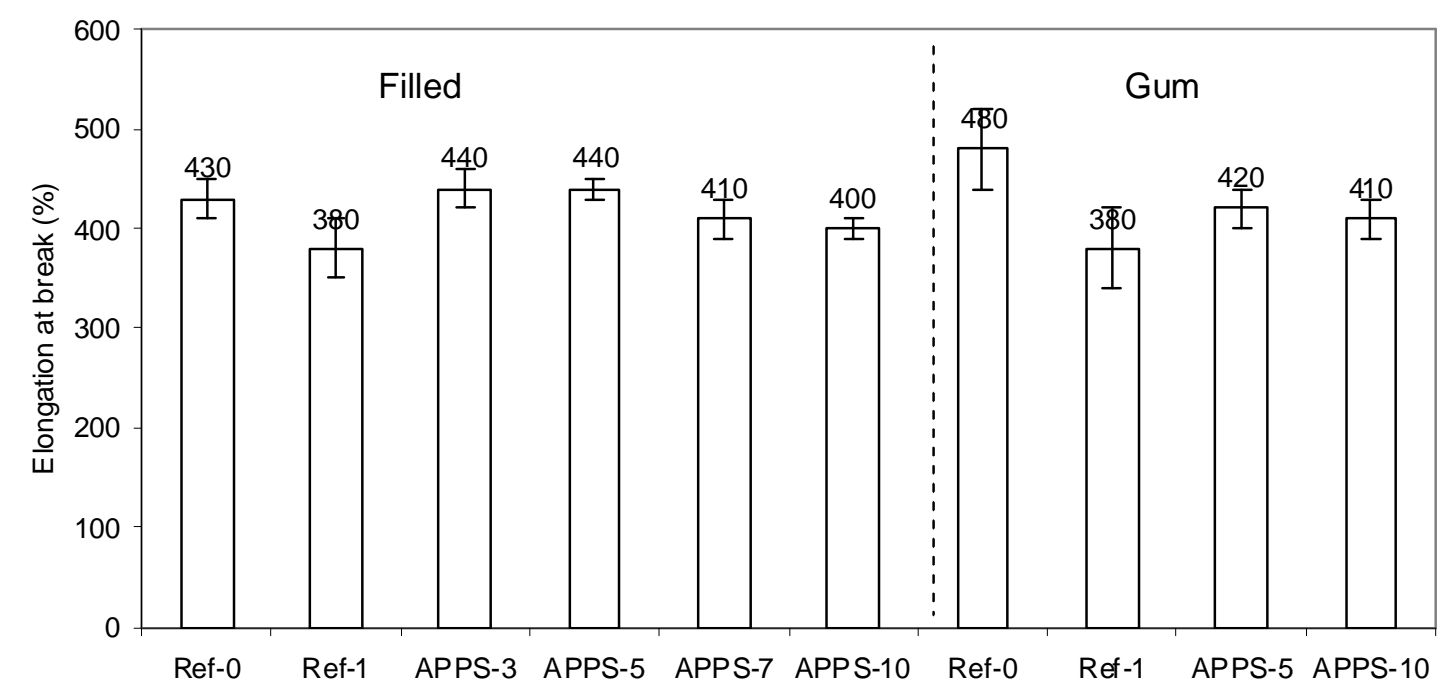

Figure 8.8 Elongation at break for NR/BR and NR/BR/(APPS-)EPDM blends.

\subsubsection{Comparison between EPDM-pretreatment and straight mixing}

From above results, it is clear that APPS-grafted EPDM leads to significant improvements in the tensile properties, especially the tensile strength. However, it is not clear if those improvements are the results of the mere presence of APPS or of the grafting of APPS onto EPDM. Therefore, a comparison was made between EPDM-pretreatment and simple straight mixing, which means that the same amount of APPS was added directly to the blend compounds. Figure 8.9 shows the tensile curves and the characteristics data of the tensile properties of Ref-1 and the blends with and without EPDM-pretreatment.

Compared to the blend with APPS-grafted EPDM, the blend by straight mixing shows much lower tensile strength and similar elongation at break. It has a similar $100 \%$ modulus, while lower $300 \%$ modulus than the blend with APPS-grafted EPDM. Compared to Ref-1, the blend by straight mixing shows higher moduli but no significant improvement of the tensile strength and elongation at break.

It demonstrates that the EPDM-pretreatment step is necessary to obtain significant improvements of the tensile properties for the NR/BR/EPDM blends. The straight addition of APPS only introduces extra sulphur into the compounds, which leads to some higher modulus values. This APPS tends to migrate to the NR and BR phases instead of to the EPDM. In that case, the presence of APPS does not overcome the cure incompatibility and will also not enhance the carbon black reinforcement of the EPDM phase to obtain a better overall filler distribution. Moreover, the extra sulphur in the NR/BR phase may result in overcure of this phase. 


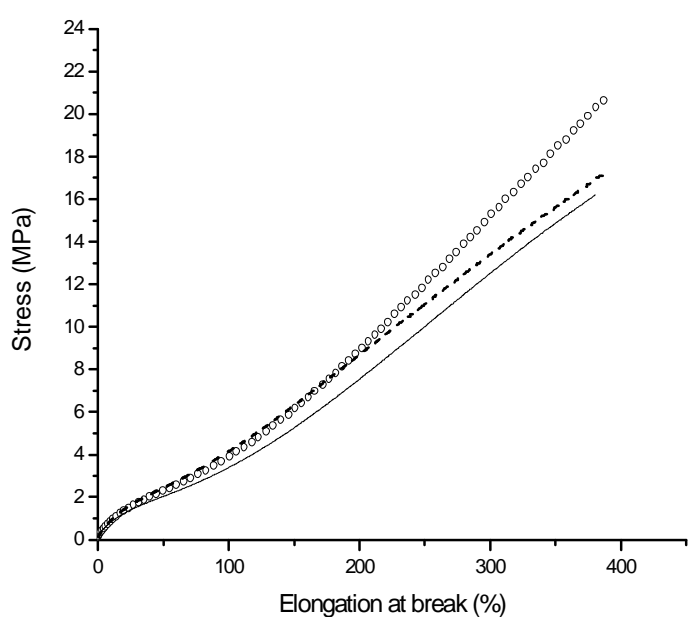

(a)

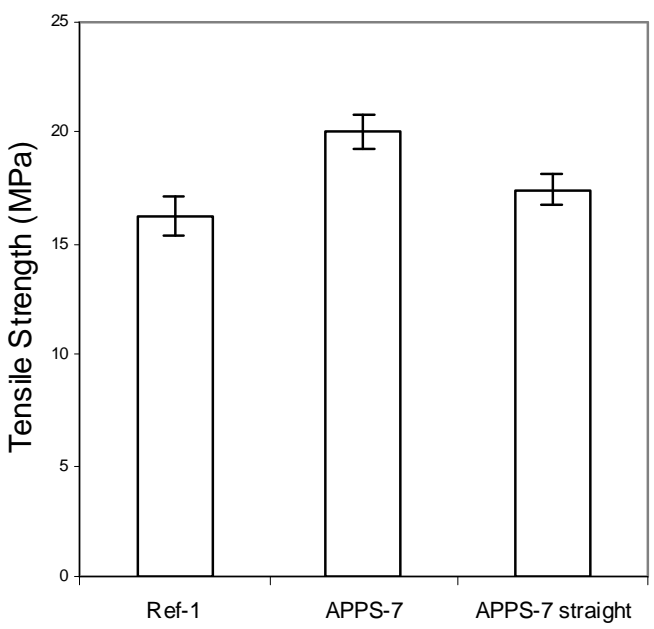

(c)

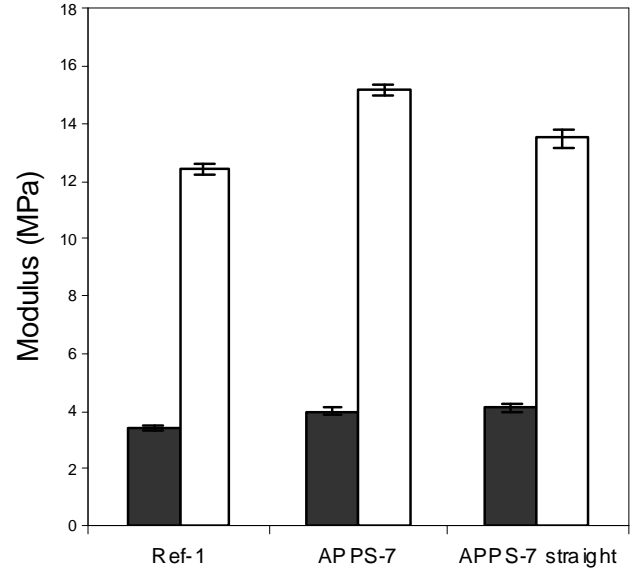

(b)

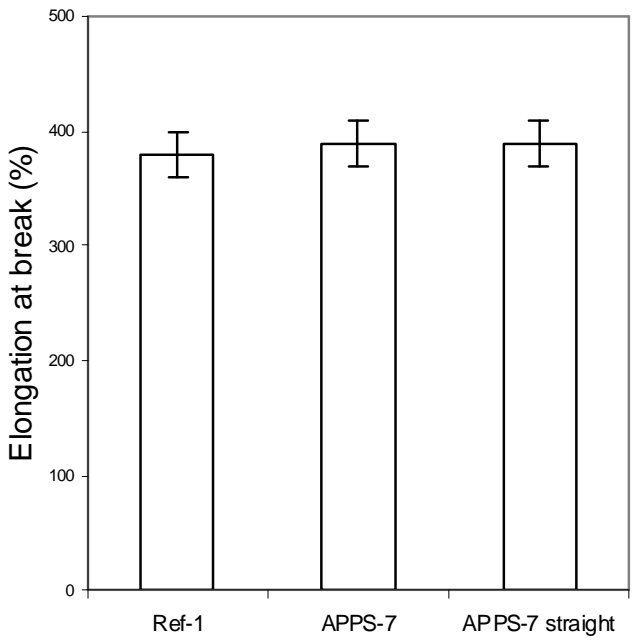

(d)

Figure 8.9 Comparison of tensile properties between Ref-1, APPS-7 and APPS-7-straight mixing: (a) tensile curves: (-): Ref-1; (oo): APPS-7; (...): APPS-7-straight; (b) Modulus: (-) at $100 \%$ strain; (口) at $300 \%$ strain; (c) Tensile strength; (d) Elongation at break.

\subsubsection{Tear Strength}

The trouser tear strengths of the HAF-filled and gum blends are shown in figure 8.10. A similar trend can be observed for the tear strength as for the tensile strength. The NR/BR blend exhibits a much higher tear strength than the blend with unmodified EPDM. When APPS-modified EPDM is applied, the tear strength increases significantly and is similar or even slightly higher than for Ref- 0 . The higher tear strength as well as the higher tensile strength of such NR/BR/EPDM blends is the result of a better homogeneity of either crosslink distribution or carbon black distribution, or both: see Chapter 9. The better dispersion of the EPDM particles inside the NR/BR matrix may also impede the tear propagation and hence increase tearing energy and tear strength. 


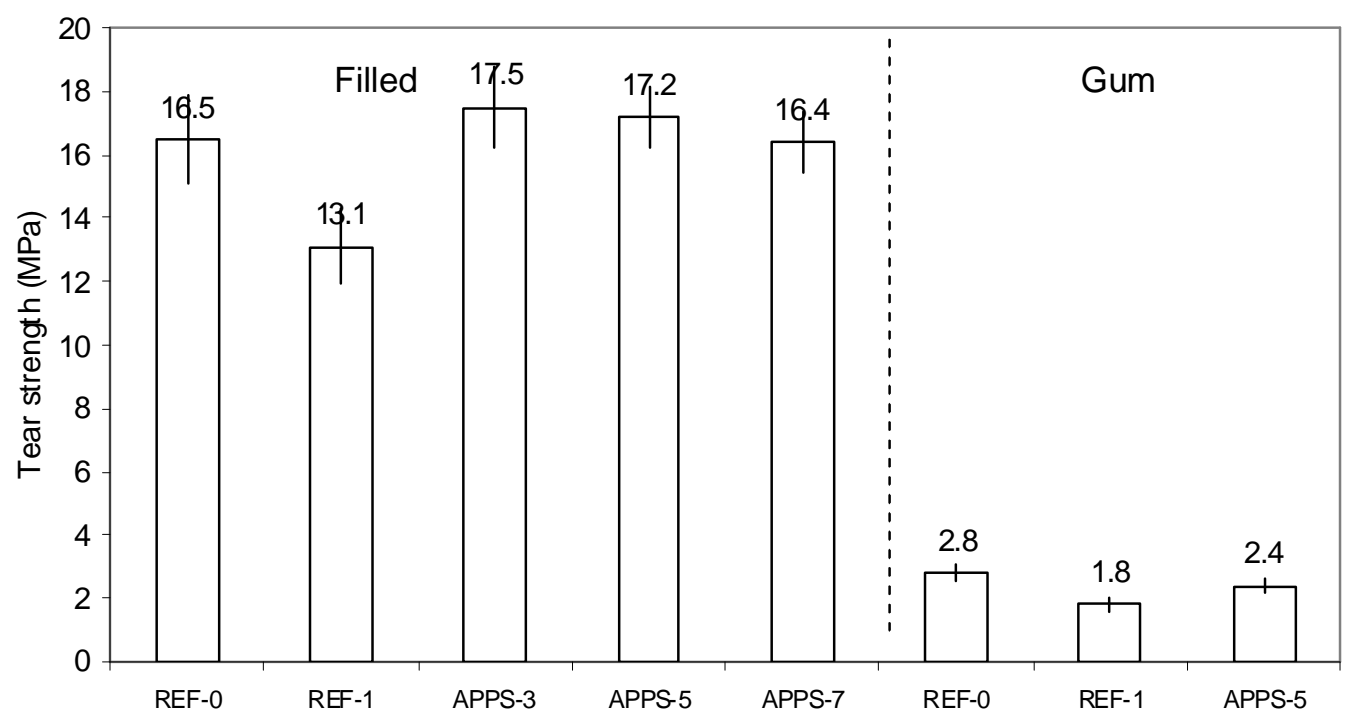

Figure 8.10 Trouser tear strength for NR/BR and NR/BR/(APPS-)EPDM blends.

\subsubsection{Fatigue to Failure}

Fatigue to failure is defined as the tendency of a material to rupture by means of progressive cracks under stress-strain cycles. As already discussed in Chapter 4, the modulus of a sample plays a very important role for the fatigue life, if based on the same extension. The reason is that, for the same extension ratio, more energy will be consumed during each cycle for higher modulus specimen and therefore, a shortened fatigue life will be obtained. Above results have shown that the NR/BR/APPS-EPDM blends have higher moduli as compared to Ref-0 and Ref-1. Therefore, in order to exclude the influence from the different modulus of the different samples, this test was carried out at the same strain energy by adjusting the fatigue extension ratio.

The fatigue lives at constant energy of the filled and gum blends are shown in figure 8.11. For both the filled and gum cases, the use of APPS-grafted EPDM leads to a large improvement in fatigue life, not only compared to Ref-1 but also in comparison with the NR/BR blends. Therefore, EPDM-modification by APPS does result in much better fatigue-to-failure properties for such a NR/BR/EPDM blend. The primary controlling parameters affecting the fatigue life are inhomogeneities or flaws present in each sample ${ }^{20}$, caused by the blending, mixing, curing and sample cutting steps. It is furthermore well known that APPS as sulphur donor can bring better dynamic fatigue properties to rubber compounds. In this study, after APPS was grafted onto EPDM, the longer fatigue life of the NR/BR/APPS-EPDM blend can also result from a better blend homogeneity because of improved co-cure, and the improvement of filler distribution among the various rubber phases in the blend: see Chapter 9. 


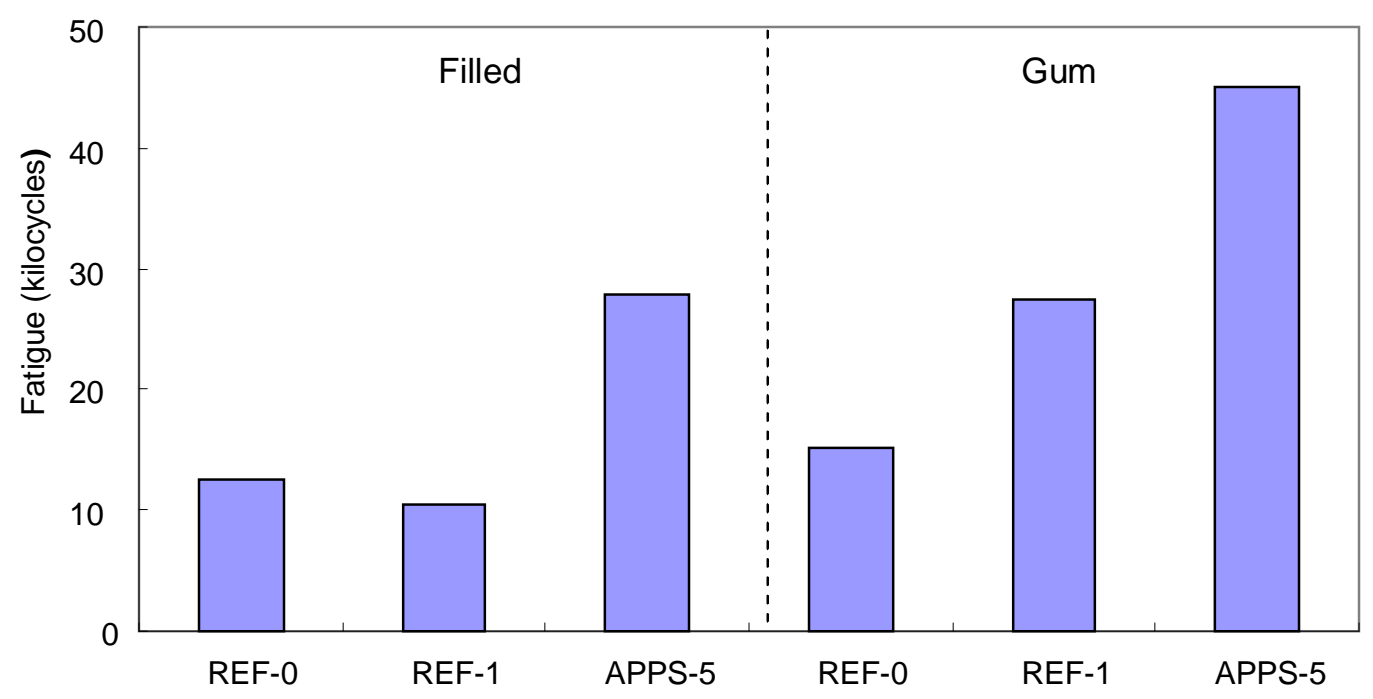

Figure 8.11 Fatigue-to-failure of the blends at constant strain energy:

$\mathrm{HAF}$-filled blends at 1.8MPa; gum blends at 1.2MPa.

\subsubsection{Dynamic viscoelastic properties}

Rolling resistance is one of the major parameters of a tyre accounting for energy consumption. The tyre sidewall contributes approximately $10 \%$ of the total rolling resistance for both passenger car tyres and truck tyres as shown in figure $8.12^{21}$. Because the dynamic viscoelastic properties are indicative for rolling resistance, they are essential parameters for tyre sidewalls.

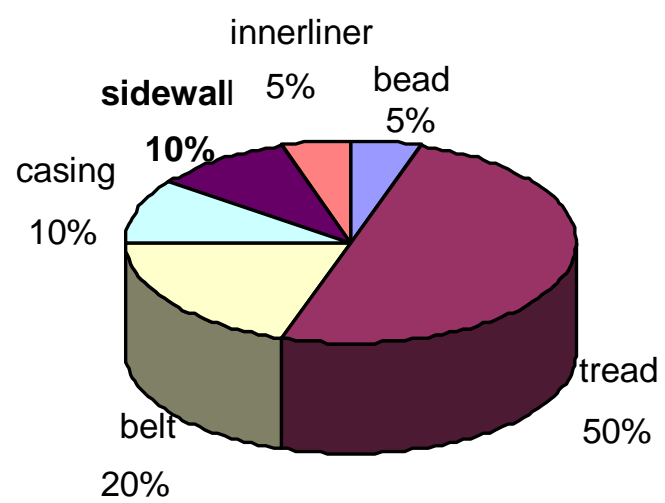

(a)

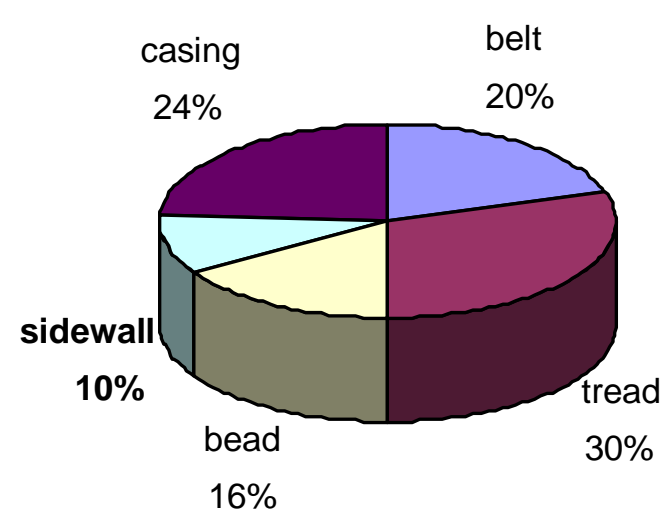

(b)

Figure 8.12 Influences on rolling resistance for (a) passenger car tyre and (b) truck tyre.

Elastomers are viscoelastic materials possessing both viscous and elastic behaviour. Therefore, upon deformation part of the energy is elastically stored, while the rest is dissipated through viscous behaviour. The loss angle $\delta$, the phase angle 
between stress and strain during oscillatory loading, and the tangent of the loss angle $\tan \delta$ can be taken as a good parameter for the viscoelastic behaviour of rubber:

$$
\operatorname{Tan} \delta=\frac{G^{\prime \prime}}{G^{\prime}}
$$

Where G" is the loss modulus and corresponds to the viscous behaviour, and G' is the storage modulus, corresponding to the elastic behaviour of the rubbers.

Figure 8.13 shows the results of frequency-sweep measurements performed at $100^{\circ} \mathrm{C}$ and $14 \%$ strain on the unvulcanised HAF-filled blends. The storage modulus of the NR/BR/APPS-grafted EPDM blend is very similar to the NR/BR blend over the whole frequency range, and is lower than the blend with unmodified EPDM. For frequencies higher than $1 \mathrm{~Hz}$, the tan $\delta$ value of NR/BR/APPS-EPDM is similar to Ref- 0 as well and higher than for Ref-1. Possible reasons of this result are that unreacted APPS can act as plasticiser for the blend; some crystallinity in unmodified EPDM may give an elastic contribution to the unvulcanised specimen.

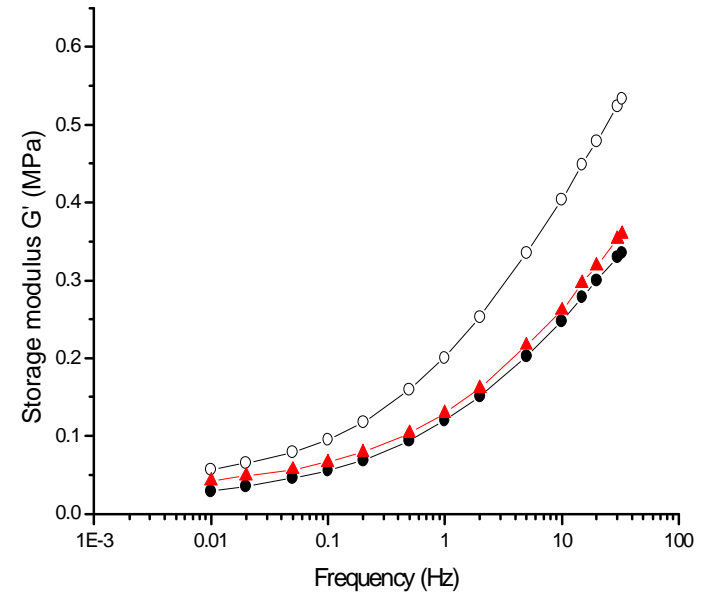

(a)

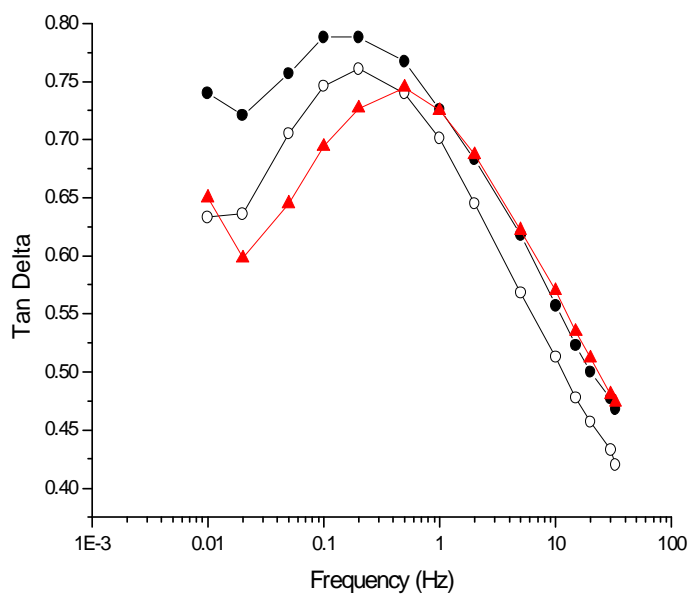

(b)

Figure 8.13 (a): Storage modulus, and (b): Tan $\delta$, of unvulcanised HAF-filled blends as function of frequency: $(\bullet)$ : Ref-0; (०): Ref-1; ( $\mathbf{\Delta})$ : APPS-5.

The results of frequency-sweep measurements performed at $60^{\circ} \mathrm{C}$ and $3.5 \%$ strain on the vulcanised HAF-filled blends are shown in figure 8.14. The storage moduli of all the blends increase with higher frequency. The increasing trend for all EPDM-containing blends is steeper than for the NR/BR blend. The difference of the storage moduli between the unmodified and grafted EPDMs is not very pronounced. The rolling resistance or tyres is commonly related to the loss tangent of rubber compounds at the low frequency region within the rubbery state. Tan $\delta$ at $60^{\circ} \mathrm{C}$ around $15 \mathrm{~Hz}$ is often taken as a good indicator for rolling resistance of a tyre. A lower $\tan \delta$ corresponds to a lower rolling resistance. The tan $\delta$ value of the NR/BR blend is 
much lower than the straight unmodified NR/BR/EPDM blend, which means that the addition of unmodified EPDM to the tyre sidewall compound not only results in poor mechanical properties but also poor dynamic properties. After EPDM-grafting by APPS, the $\tan \delta$ value is still some higher than the NR/BR blend. However, compared to Ref-1, a significant improvement can again be found as a pronounced decrease of the tan $\delta$ value, indicative for a lower rolling resistance. For different APPS-amounts, 5wt\% APPS-modified EPDM shows the lowest tan $\delta$, but the difference is not very great.

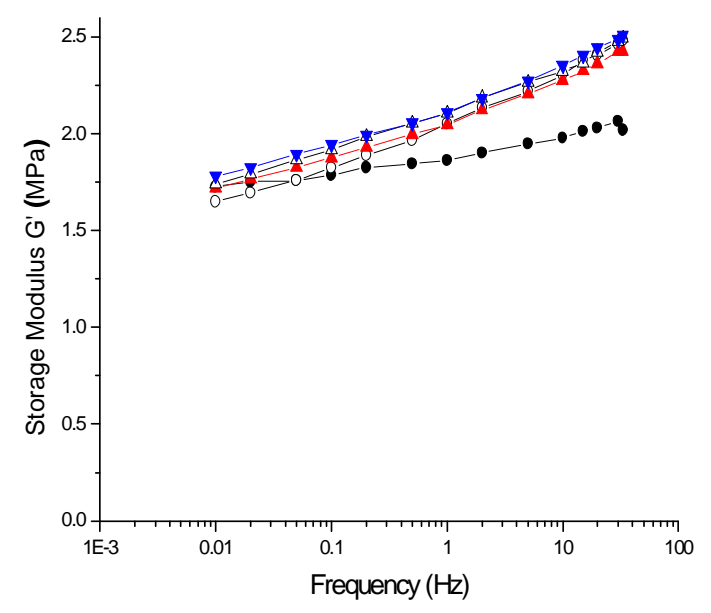

(a)

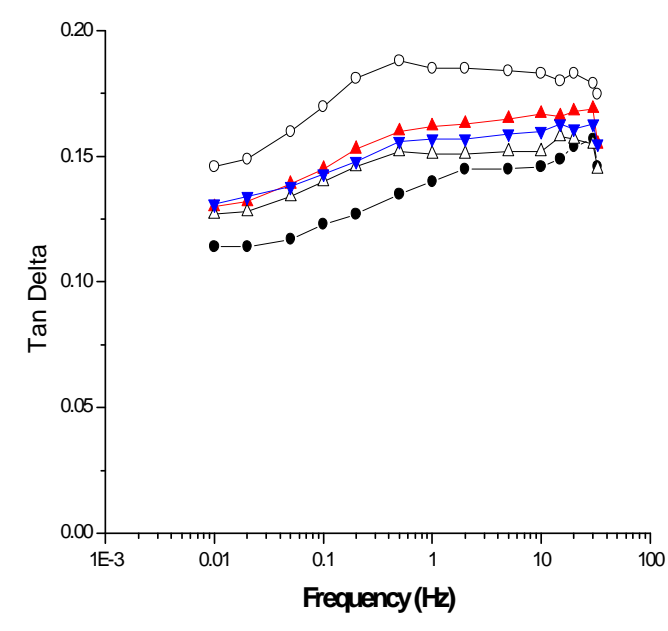

(b)

Figure 8.14 (a): Storage modulus and, (b) Tan $\delta$, of vulcanised HAF-filled blends as function of frequency: $(\bullet)$ : Ref-0; (०): Ref-1; ( $\mathbf{\Delta})$ : APPS-3; $(\square)$ : APPS-5; $(\boldsymbol{\nabla})$ : APPS-7.

\subsubsection{Ageing behaviours}

The rubber constituents of a tyre age during service. Ageing causes unwanted changes in product performance and eventually shortens the service life. Therefore, protection against ageing is one of the important design items for the tyre and for the sidewall in particular.

Ageing of rubber vulcanisates changes the network structure by crosslink scission and desulphurisation of polysulphidic bonds into mono- and/or disulphidic crosslinks. Heat increases the diffusion of oxygen and accelerates the oxidation of rubbers. Figure 8.15 shows the influence of ageing time at $70^{\circ} \mathrm{C}$ on the tensile properties of gum Ref-0, Ref- 1 and APPS- 5 blends. The modulus increases for all blends, while tensile strength and elongation at break decrease. It can be observed that the properties change most significantly during the first day throughout the whole testing time period. As all the compounds were vulcanised for optimum time of $t_{90}$, some further vulcanisation can still take place on the first day. Moreover, it is known that desulphurisation takes place most pronouced on the first day of ageing for natural rubber compounds. Therefore, the real oxidative ageing behaviour of the 
rubber blends should be considered after one day ageing. The relative changes of the properties of the blends after 30 days ageing can then be calculated by the following equation:

$$
\text { Relative-change }{ }_{30}=\frac{X_{30}-X_{1}}{X_{1}} * 100 \%
$$

where $X_{1}$ is the value of the property after one day of ageing; $X_{30}$ is the value of the property after 30 days of ageing.

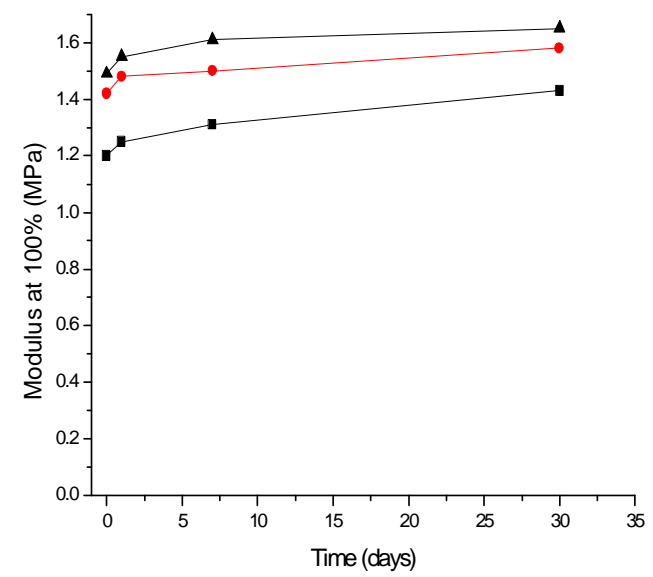

(a)

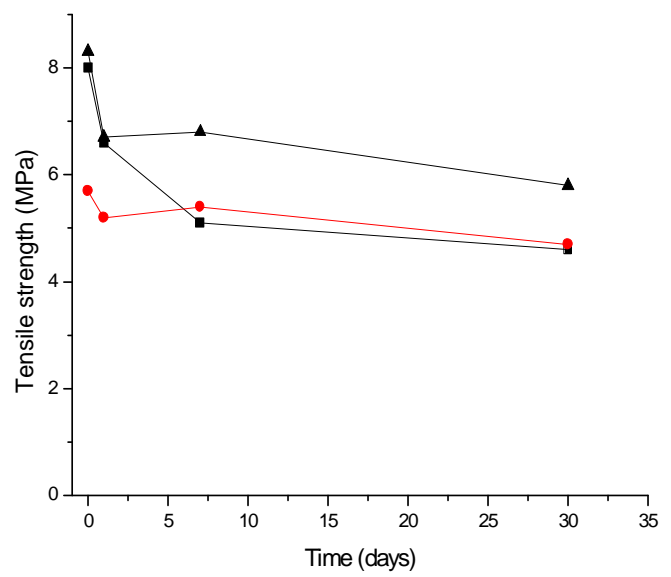

(b)

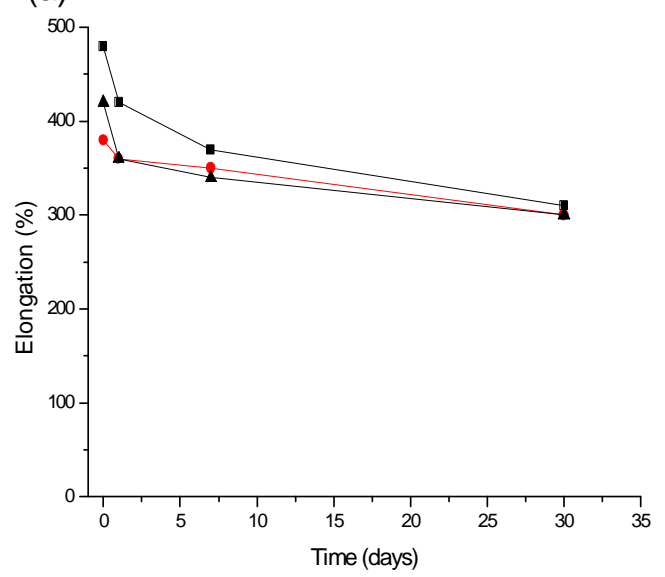

(c)

Figure 8.15 Influence of ageing time at $70^{\circ} \mathrm{C}$ on the tensile properties for gum blends (a) modulus at 100\%; (b) tensile strength; (c) elongation at break: (घ): Ref-0; (•): Ref$1 ;(\mathbf{\Lambda})$ : APPS-5.

The relative changes of the tensile properties for the gum blends are shown in Table 8.2. Ref-0 has a higher change of modulus, tensile strength and elongation at break compared to Ref- 1 and APPS-5. It is well known that EPDM has a better heat resistance than NR and $\mathrm{BR}$, because of its saturated polymer backbone. Therefore, both blends with 30phr EPDM also show better heat resistance than the simple 
NR/BR blend. The difference of the ageing behaviours between the blends with virgin EPDM and APPS-grafted EPDM are not considered significant.

Table 8.2 Relative changes of the tensile properties for the gum blends after 30 days vs. properties after 1 day ageing (\%).

\begin{tabular}{l|ccc}
\hline & Modulus at $100 \%$ & Tensile strength & Elongation at break \\
\hline Ref-0 & +14.4 & -30.3 & -26.2 \\
Ref-1 & +6.8 & -9.8 & -16.7 \\
APPS-5 & +6.5 & -13.1 & -16.7 \\
\hline
\end{tabular}

A similar trend for the ageing properties of the filled blends can be observed like for the gum blends $\square$ figure 8.16. The relative changes of the tensile properties for the filled blends vs. the 1 day values are calculated according to eq 8.1 and listed in Table 8.3. The modulus of Ref-0 increases more significantly than for the other EPDM-containing blends. The changes of tensile strength and elongation at break are more or less the same for all the blends.

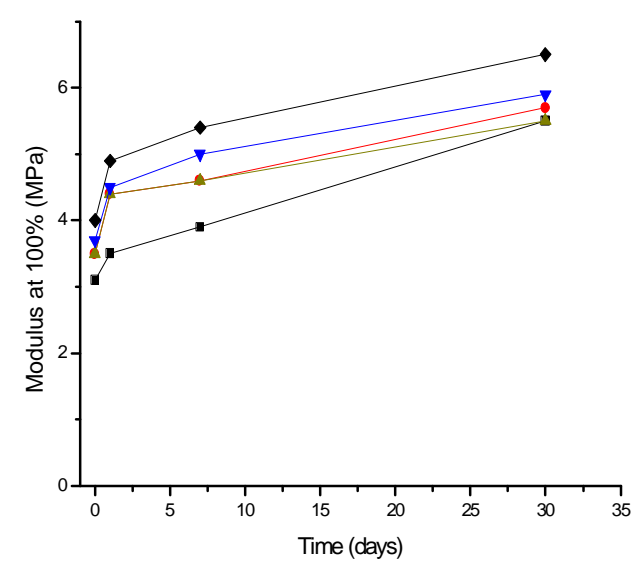

(a)

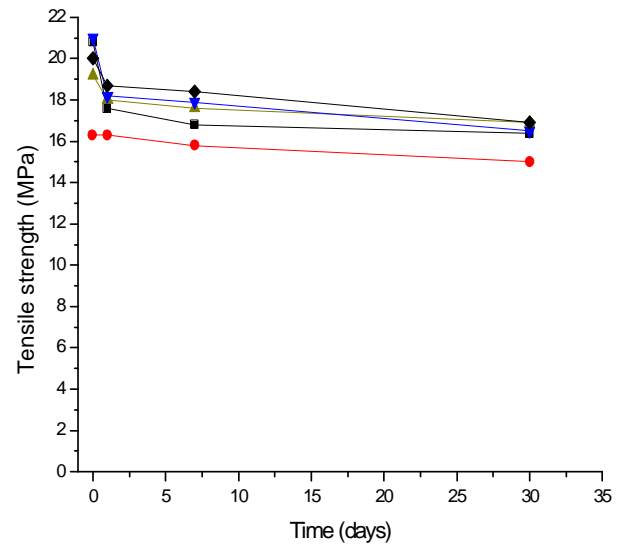

(b)

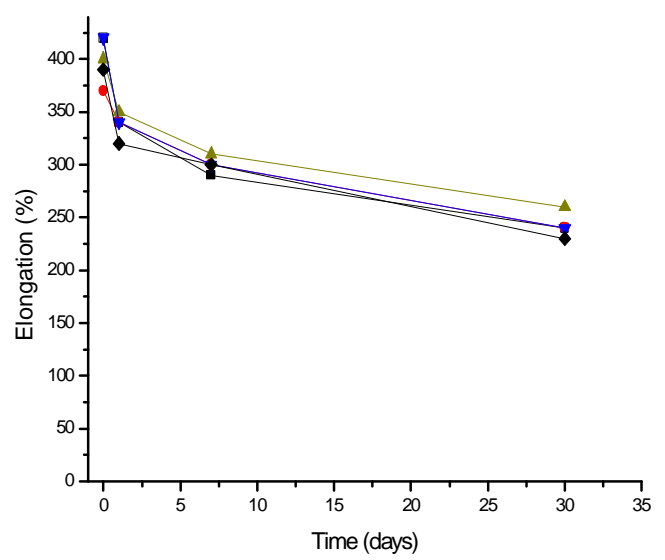

(c)

Figure 8.16 Influence of ageing time at $70^{\circ} \mathrm{C}$ on the tensile properties for HAF-filled blends:

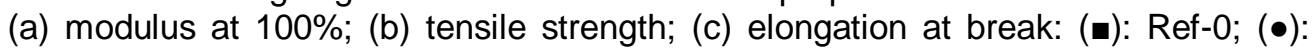
Ref-1; ( $\mathbf{\Delta})$ : APPS-3; $(\mathbf{\nabla})$ : APPS-5; ( $\square$ ): APPS-7. 
Table 8.3 Relative changes for the tensile properties for the filled blends after 30 days vs. properties after 1 day ageing (\%).

\begin{tabular}{l|lll}
\hline & Modulus at $100 \%$ & Tensile strength & Elongation at break \\
\hline Ref-0 & +57 & -6.8 & -29 \\
Ref-1 & +30 & -4.8 & -29 \\
APPS-3 & +26 & -7.1 & -27 \\
APPS-5 & +31 & -9.3 & -29 \\
APPS-7 & +32 & -9.6 & -28 \\
\hline
\end{tabular}

It has been observed that the use of alkylphenol-disulphide as curing agent can lead to improved ageing properties due to an increased number of monosulphidic crosslinks ${ }^{17}$. However, the NR/BR/APPS-EPDM blend does not show an improvement relative to Ref-1. EPDM by itself has very good ageing properties compared to NR and BR. Therefore, in the NR/BR/EPDM blend the part which needs to be improved with respect to the ageing properties is the NR/BR-phase. In this study, the APPS was pre-reacted with the EPDM as modification agent, so most of the APPS was confined in the EPDM phase. Even if there would be some rests of APPS left, which had not been grafted onto EPDM and might migrate to the NR/BR phase, their amounts are not enough to lead to a significant improvement of the ageing properties of the blends. Because both NR/BR/EPDM and NR/BR/APPSEPDM blends show better ageing properties than the NR/BR blend, it is clear that the improvement is coming from the EPDM-addition, with or without APPS grafted thereon.

\subsection{Conclusions}

The physical properties of the NR/BR/APPS-EPDM blends investigated are much better compared with compounds using virgin EPDM, and are equivalent or even superior to conventional NR/BR tyre sidewall compounds. The tensile strength and tear strength of the NR/BR/APPS-EPDM blends and the reference NR/BR tyre sidewall compound are more or less equivalent. The straight addition of APPS to the NR/BR/EPDM blend does not lead to significant improvements of the tensile properties. Therefore, pretreatment of EPDM with APPS is a necessary step. The NR/BR/APPS-EPDM blends show superior fatigue-to-failure properties to straight $\mathrm{NR} / \mathrm{BR} / \mathrm{EPDM}$ and the NR/BR tyre sidewall compound. Dynamic viscoelastic properties are also improved by using APPS-grafted EPDM compared to virgin EPDM, although there is still room for improvement relative to the conventional NR/BR sidewall blend. All NR/BR/EPDM blends show better ageing properties 
relative to the NR/BR compound, while the use of APPS-grafted EPDM does not result in further improvement relative to straight EPDM.

\section{REFERENCES}

1. Chapman, A. V.; Tinker, A. J., Kautsch. Gummi Kunstst., 56, 533 (2003).

2. Ignatz-Hoover, F.; To, B. H.; Datta, R. N.; Hoog, A. J. d.; Huntink, N. M.; Talma, A. G., Rubber Chem. Technol., 76, 747 (2003).

3. Guillaumond, F. X., Revue Generale des Caoutchoucs et Plastiques, 51, 853 (1974).

4. Klüppel, M.; Schuster, R. H.; Schaper J., Rubber Chem. Technol., 72, 91 (1999).

5. van de Ven, P. M.; Noordermeer, J. W. M., Rubber World, 222, 55 (2000).

6. Zhao, J.; Ghebremeskel, G., Kautsch. Gummi Kunstst., 3, 84 (2001).

7. Woods, M. E.; Davidson, J. A., Rubber Chem. Technol., 49, 112 (1976).

8. Coran, A. Y., Rubber Chem. Technol., 61, 281 (1988).

9. Oliveira, M. G.; Soares, B. G., Macromol. Rapid Commun., 20, 526 (1999).

10. Baranwal, K. C.; Son, P. N., Rubber Chem. Technol., 47, 88 (1974).

11. Morrissey, R. T., Meeting of the Rubber Division, American Chemical Society, Miami, Florida, April, 1971.

12. Hashimoto, K.; Miura, M.; Takagi, S.; Okamoto, H., Int. Polym. Sci. Technol., 3, T84 (1976).

13. Cook, S., in "Blends of Natural Rubber", Chapman \& Hall, London, 1998.

14. Hopper, R. J., Rubber Chem. Technol., 49, 341 (1976).

15. Zapp, R. L., Rubber Chem. Technol., 46, 251 (1973).

16. Rodgers, M. B.; Solis, S. C.; Tambe, N.; Sharma, B. B., Rubber Chem. Technol., 81, 600 (2008).

17. Solis, S. C.; Rodgers, M. B.; Tambe, N.; Sharma, B. B.; Waddell, W. H., Meeting of the Rubber Division, American Chemical Society, San Antonio, Texas, October, 2005.

18. Flowers, D. D.; Fusco, J. V.; Tracey, D. S., Rubber World, 209, 32 (1994).

19. Monsanto technical service report No. 84-078, 1984.

20. Young, D. G.; Kresge, E. N.; Wallace, A. J., Rubber Chem. Technol., 55, 428 (1982).

21. Chang, L. Y.; Schackleton, J. S., Meeting of the Rubber Division, American Chemical Society, Chicago, Illinois, October, 1982. 


\title{
Chapter 9
}

\section{Modification of EPDM with Alkylphenol Polysulphide for \\ Use in Tyre Sidewalls: Part II. Mechanistic and \\ Morphological Characterisations}

\begin{abstract}
Cure incompatibility, and heterogeneous filler distribution result in poor mechanical properties of NR/BR/EPDM blends. In the first part of this study, it has been shown that the mechanical properties of such a blend can be significantly improved when APPS-graftedEPDM is used instead of straight EPDM. In the present chapter, the infrared investigation shows that modification of EPDM with APPS takes place and saturation occurs when the APPS amount reaches $5 w t \%$. A better rubber-carbon black affinity by using APPS-grafted EPDM in the blend can be proved from bound rubber and carbon black-adsorption measurements. TEM and STEM images clearly show that the NR/BR/APPS-EPDM blend has a significantly more homogeneous rubber phases morphology compared to the straight NR/BR/EPDM blend. A significantly better distribution of the carbon black even into the APPS-EPDM phase is mainly held responsible for the improvement in properties of the blend.
\end{abstract}




\subsection{Introduction}

Alkylphenol disulphide (APPS) is commonly considered a sulphur donor for curing of elastomers. It is particularly recommended in butyl and halobutyl rubber based applications ${ }^{1-4}$, with benefits as being nitrosamine-free, not blooming, providing good heat resistance, improved dynamic fatigue properties, enhanced adhesion and functioning as tackifier.

In Chapter 8 APPS has been used to modify EPDM in order to solve the cure incompatibility and heterogeneous filler distribution of NR/BR/EPDM blends for tire sidewall applications. In the first part of this study as described in Chapter 8, it was shown that the application of APPS-grafted EPDM to the blends leads to a significant improvement of the tensile properties, tear strength and fatigue properties compared to using virgin EPDM. Those physical properties of the NR/BR/APPSEPDM blends are equivalent or even superior to conventional NR/BR tire sidewall compounds. Dynamic viscoelastic properties are also improved by using APPSgrafted EPDM compared to using virgin EPDM $\square$ It has also been found that the straight addition of APPS to the NR/BR/EPDM blend does not lead to similar improvements of the tensile properties as the blend with APPS-pretreated EPDM, indicating that grafting of APPS onto EPDM is an essential requirement for those improved properties.

Due to the higher polarity of APPS, using it as modification agent may introduce higher polarity into the EPDM, which may enhance partitioning of more curatives and carbon black filler from the NR/BR phase to the EPDM phase, and also improve the compatibility of EPDM with NR and BR. In this part of the work, the objective is to study the mechanisms involved and to create a better understanding of the significant improvement of mechanical properties of the NR/BR/APPS-EPDM blends. A calibration of the grafting efficiency of APPS onto EPDM is studied first, based on the ATR/IR spectrum. Bound rubber content measurements and carbon black adsorption experiments are done to create a better idea about the influence of APPS on the polymer-carbon black interaction. In addition, the morphological features of the blends are studied by using the TEM and STEM techniques.

\subsection{Experimental}

\subsubsection{Materials}

The blends consist of the same ingredients as mentioned in Chapter 8.

Additional chemicals used in the carbon black adsorption experiments are phenyl disulphide (99\%, Aldrich) and phenol disulphide (4,4'-Thioldiphenol, 99\%, Aldrich). 
Staining agent for the TEM experiment was a $2 \%$ osmic acid solution in water (Merck).

\subsubsection{Grafting of APPS onto EPDM}

Grafting of APPS onto EPDM was done in a Brabender PL2000 as described in Chapter 8. The grafted EPDM is still soluble in common solvents, as chloroform, toluene and hexane, indicating that no un-wanted crosslinking has occured. The sample for ATR/IR analysis was then extracted by acetone for 24 hours to remove unreacted chemicals, followed by drying in a vacuum oven.

\subsubsection{Characterization of the grafted EPDM by ATR/IR}

The infrared spectra of the grafted EPDM-samples were recorded by using the attenuated total reflectance infrared technique (ATR/IR) with a Perkin Elmer Spectrum 100. The infrared spectra were recorded with 12 scans at a resolution of $4 \mathrm{~cm}^{-1}$ in the range of $4000-600 \mathrm{~cm}^{-1}$.

\subsubsection{Preparation of the blends and vulcanisation}

Preparation of the blends and vulcanisation was the same as described in Chapter 8. The overall formulations of the blends are shown in Table 9.1.

Table 9.1 Formulations of NR/BR/EPDM (grafted-EPDM) compounds

\begin{tabular}{l|cc}
\hline Ingredients (phr) & Ref-1 & ${\text { APPS- }{ }^{*}}^{*}$ \\
\hline NR SIR 20 & 35 & 35 \\
BR Kosyn KBR01 & 35 & 35 \\
EPDM Keltan 5508 & 30 & - \\
APPS-EPDM & - & $30+0.3^{*} \mathrm{X}$ \\
Zinc Oxide & 4 & 4 \\
Stearic Acid & 2 & 2 \\
TMQ & 1.98 & 1 \\
CBS & 2.5 & 2.5 \\
Sulphur & 50 & 50 \\
Carbon black HAF N330 & 10 & 10 \\
\hline & Oil $\mathrm{X}=$ Amount of APPS in wt\% used for modification of $100 \mathrm{wt} \%$ EPDM
\end{tabular}




\subsubsection{Bound rubber measurement}

The bound rubber content was measured for unvulcanised rubber compounds. $0.2 \mathrm{~g}$ unvulcanised samples were cut into small pieces and put into a steel-wire basket of very fine mesh, which was immersed in $100 \mathrm{ml}$ of toluene at room temperature for 72 hours. The solvent was renewed every 24 hours. The extracts were collected and left for $\mathbf{2 4}$ hours in air, followed by drying in a vacuum oven at $1050 \mathrm{C}$ to evaporate the solvent.

The amount of bound rubber (BDR) is expressed as the percentage of the total polymer content in the compound and is calculated according to the following equations:

$$
\begin{aligned}
& m_{0}=\left(m_{2}-m_{1}\right) * \frac{100}{m} \\
& \operatorname{BDR}(\%)=\frac{m_{0}-\left(m_{2}-m_{3}\right)}{m_{0}} * 100 \%
\end{aligned}
$$

Where $m_{0}$ is the rubber content in the sample, $m_{1}$ is the weight of the basket, $m_{2}$ is the collective weight of the basket and unextracted material, $m_{3}$ is the collective weight of the basket and extracted dried material and $m$ is the total formulation (in phr) of the compound.

\subsubsection{Carbon black adsorption measurement}

The adsorption by carbon black of three different chemicals: phenyl disulphide, phenol disulphide and APPS, was carried out in solution. $0.2 \mathrm{~g}$ carbon black was added to $10 \mathrm{ml}$ of an acetone solution with different chemical concentrations: from 0.02 to $0.1 \mathrm{~g} / \mathrm{ml}$, in a centrifuge tube. The tube was shaken continously for 24 hours to insure a good suspension of the carbon black in the solution. The carbon black with adsorbed chemical was then isolated by centrifuging (Eppendorf Centrifuge 5804), followed by drying in a vacuum oven. The adsorption values of the chemicals on carbon black were calculated from the weight increase of the carbon black.

\subsubsection{Transmission Electron Microscopy (TEM) and Scanning Transmission Electron Microscopy (STEM)}

$100 \mathrm{~nm}$ thin sections of the blend vulcanisates were cryogenically cut with a diamond knife using a Leica ultra microtome at $-100^{\circ} \mathrm{C}$. The sections were deposited 
on a carbon support film on copper grid (200 mesh) and then vapour stained using $2 \%$ osmium tetroxide for 10 minutes.

TEM measurements were performed with a Tecnai 20 (FEI Company, the Netherlands). STEM measurements were performed with a Titan apparatus (FEI Company, the Netherlands).

\subsection{Results and Discussion}

\subsubsection{Characterisation of APPS-grafted EPDM by ATR/IR}

Figure 9.1 shows the ATR infrared spectra for APPS-grafted EPDM before and after acetone extraction in comparison with virgin EPDM. Both un-extracted and extracted APPS-grafted EPDM clearly show additional bands compared to virgin EPDM at $3400 \mathrm{~cm}-1,1270 \mathrm{~cm}-1,1241 \mathrm{~cm}-1,1208 \mathrm{~cm}-1,1165 \mathrm{~cm}-1,1048 \mathrm{~cm}-1$, $1018 \mathrm{~cm}-1,821 \mathrm{~cm}-1$ and $670 \mathrm{~cm}-1$. The assignment of these characteristic peaks is shown in Table 9.2 on the basis of relevant literature data5,6. The samples after extraction with acetone still show the presence of above absorption peaks, which indicates that APPS-fragments are indeed grafted onto EPDM after reaction in the internal mixer. But compared to the un-extracted APPS-grafted EPDM, the intensity of these peaks decreases somewhat after extraction, which means that not all the APPS added was indeed grafted.

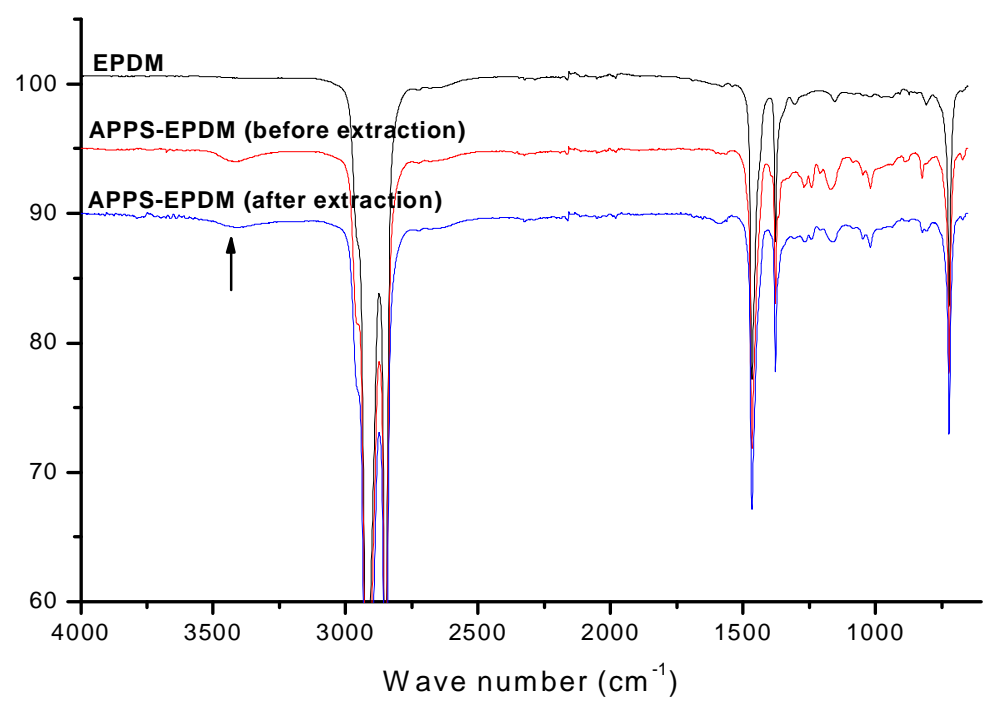




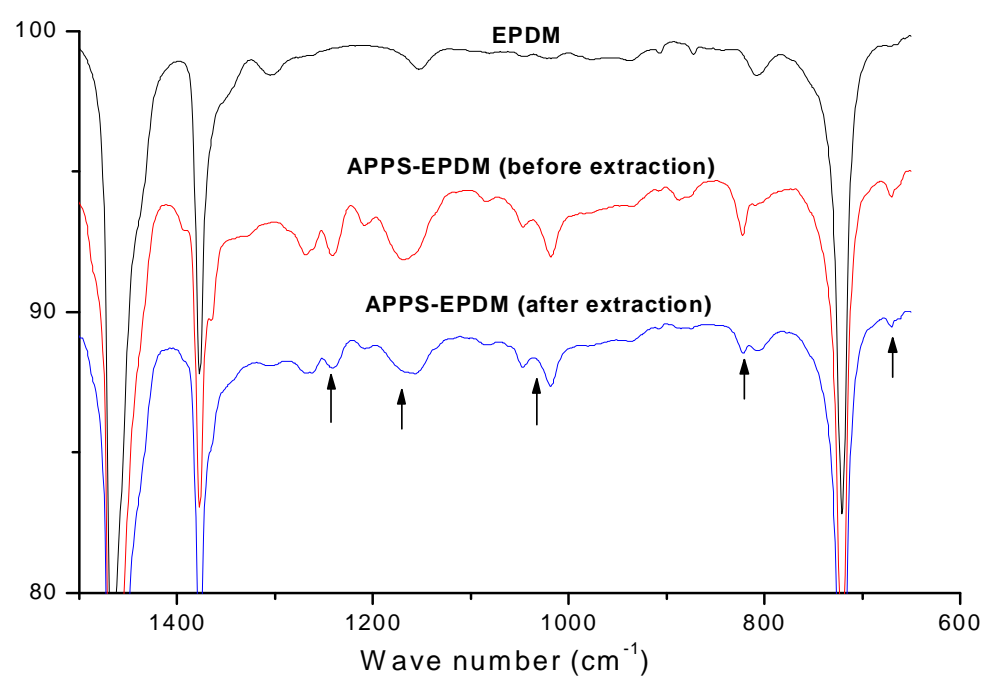

Figure 9.1 ATR/IR spectrum of EPDM, APPS-grafted EPDM before and after extraction.

Table 9.2 Assignment of the bands in the ATR/IR spectra for APPS-grafted EPDM.

\begin{tabular}{cc}
\hline Wavenumber $\left(\mathrm{cm}^{-1}\right)$ & Assignment \\
\hline 3400 & O-H stretching \\
1241 & Phenolic $\mathrm{C}-\mathrm{OH}$, asymmetric stretching \\
1169 & $\mathrm{C}-\mathrm{H}$ aromatic, in-plane \\
1048 & $\begin{array}{c}\text { Single bond } \mathrm{C}-\mathrm{O} \text { stretching } \\
\text { Vibrations of }-\mathrm{C}-\mathrm{OH} \text { group } \\
\end{array}$ \\
1018 & $\mathrm{O}-\mathrm{H}$ \\
821 & $\mathrm{C}-\mathrm{H}$ aromatic, out of plane \\
670 & Ring bend \\
\hline
\end{tabular}

A calibration study was then carried out to obtain quantitative information about the efficiency of the grafting reaction. Not all above mentioned bands are suitable for calibration purposes: for example, the $\mathrm{O}-\mathrm{H}$ stretching band at $3400 \mathrm{~cm}-1$ is too broad and the intensity is weak; and the $\mathrm{C}-\mathrm{H}$ aromatic bands at $1169 \mathrm{~cm}-1$ and $821 \mathrm{~cm}-1$ overlap with the original bands of EPDM. Two peaks at $1048 \mathrm{~cm}-1$ and 1018 $\mathrm{cm}-1$ overlap as well, and their intensities are influenced by the intermolecular hydrogen bond. The ring bend at $670 \mathrm{~cm}-1$ is also too weak. Therefore, the phenolic band at $1241 \mathrm{~cm}-1$ was finally selected as the characteristic band for the grafting efficiency, and the band at $1377 \mathrm{~cm}-1$ from the virgin EPDM taken as reference peak. Then the relative intensity of the phenolic band at $1241 \mathrm{~cm}-1\left(\mathrm{RI}_{1241}\right)$ can be calculated according to the following equation: 


$$
\mathrm{RI}_{1241}=\frac{\text { Intensity }_{\text {band1241 }}}{\text { Intensity }_{\text {band } 1377}} * 100 \%
$$

Figure 9.2 shows the results for $\mathrm{Rl}_{1241}$ versus different APPS-amounts added before and after extraction. Before extraction, the $\mathrm{Rl}_{1241}$ value increases continuously with increasing APPS-amount added, and follows a linear relation. The calibration equation is as follows:

$$
\mathrm{RI}_{1241}=3.0+3.2 *(\text { amount of APPS })
$$

The calibration curve does not exactly pass through the zero point as theoretically expected, most probably due to an inevitable error during the measurement caused by the partial overlap with an adjacent band. But this result still can be used for the estimation of the grafting efficiency. The $\mathrm{Rl}_{1241}$ values after extraction, show a different trend as compared to the results before extraction. It increases in the low APPS-amount range and reaches a plateau when the APPSamount is higher than $5 w t \%$. In the other words, the grafting of APPS onto EPDM has a critical saturated point; no more grafting can be achieved after that point even with higher APPS-amounts. Based on calculation of the stoichiometry of a possible reaction of the APPS with the ENB-amount in the EPDM, about double quantities of APPS should have reacted. So a limited grafting is most probably due to the limited solubility of APPS in EPDM.

Based on the calibration equation: eq 9.4, the grafting efficiency and the real grafted APPS-amounts can be calculated, and the results are shown in Table 9.3. It can be observed that the highest APPS-amount which indeed is grafted onto EPDM is about $3.8 w t \%$, when the added APPS-amount is $5 w t \%$ or more. 


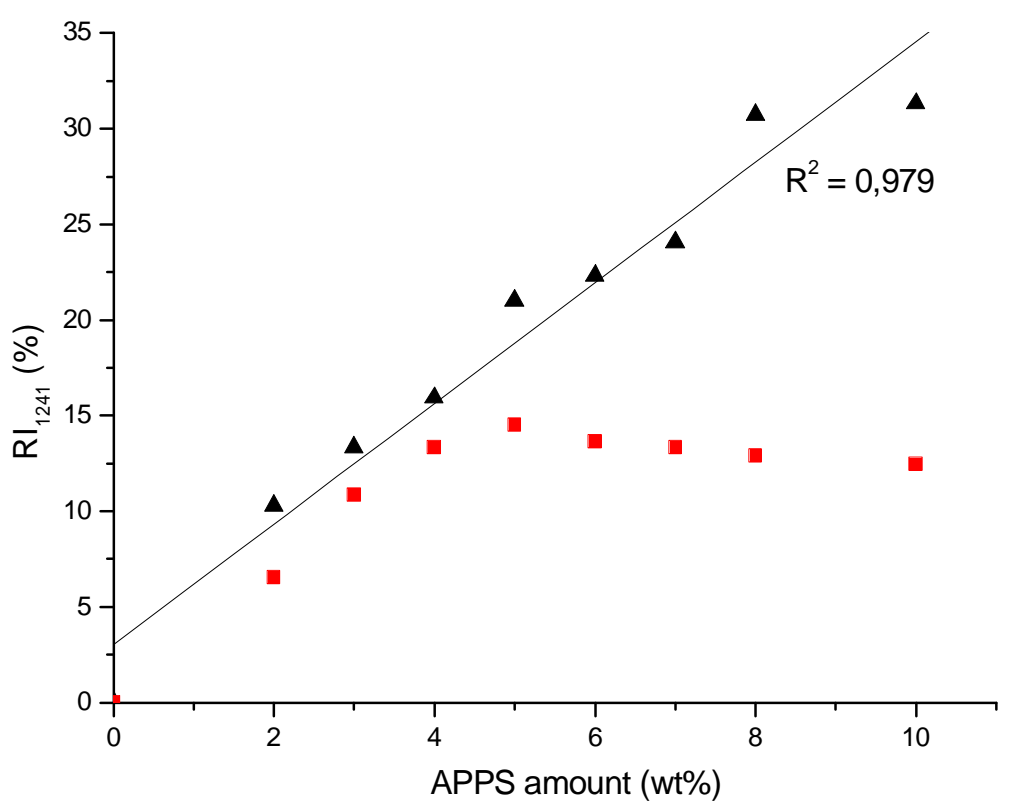

Figure 9.2 Calibration curves for APPS-grafted EPDM:

$(\boldsymbol{\Delta})$ before extraction, ( $\mathbf{-}$ )after extraction.

Table 9.3 Grafting efficiency for the reaction with different APPS-amounts.

\begin{tabular}{ccccc}
\hline $\begin{array}{c}\text { APPS-amounts } \\
(\text { wt\% })\end{array}$ & $\begin{array}{c}\mathrm{RI}_{1241}(\%) \\
\text { Theoretical }\end{array}$ & $\begin{array}{c}\mathrm{Rl}_{1241}(\%) \\
\text { Experimental }\end{array}$ & $\begin{array}{c}\text { Grafting } \\
\text { efficiency (\%) }\end{array}$ & $\begin{array}{c}\text { Real grafted } \\
\text { APPS-amounts } \\
(\mathrm{wt} \%)\end{array}$ \\
\hline 2 & 9.4 & 6,5 & $69 \%$ & 1.4 \\
3 & 12.6 & 10,9 & $86 \%$ & 2.6 \\
4 & 15.8 & 13,3 & $84 \%$ & 3.4 \\
5 & 19.0 & 14,5 & $76 \%$ & 3.8 \\
6 & 22.2 & 13,6 & $61 \%$ & 3.7 \\
7 & 25.4 & 13,3 & $52 \%$ & 3.6 \\
8 & 28.6 & 12,9 & $45 \%$ & 3.6 \\
10 & 35.0 & 12,6 & $36 \%$ & 3.6 \\
\hline
\end{tabular}

\subsubsection{Bound rubber and carbon black-rubber interaction}

If an unvulcanised carbon black-filled rubber is extracted with a good solvent, part of the rubber appears to be insoluble. This fraction is called the bound rubber, caused by the chemical and physical interactions between the carbon black particles and the rubber7-9.

Figure 9.3 shows the bound rubber contents for the NR/BR/EPDM blend and the NR/BR/APPS-EPDM blend in which EPDM is grafted by $5 w t \%$ APPS. It is clear that for EPDM grafted with APPS the bound rubber content increases significantly, 
indicating a better carbon black-rubber interaction. A possible reason is that after APPS-grafting, EPDM rubber has a much better interaction with carbon black and, consequently, more carbon black has partitioned into the EPDM phase due to the higher polarity being introduced by APPS. On the other hand, because not all the APPS added was grafted onto EPDM, as seen from the ATR calibration, part of the un-reacted APPS may have migrated into the NR/BR phase, which also enhances the carbon black-NR/BR interaction.

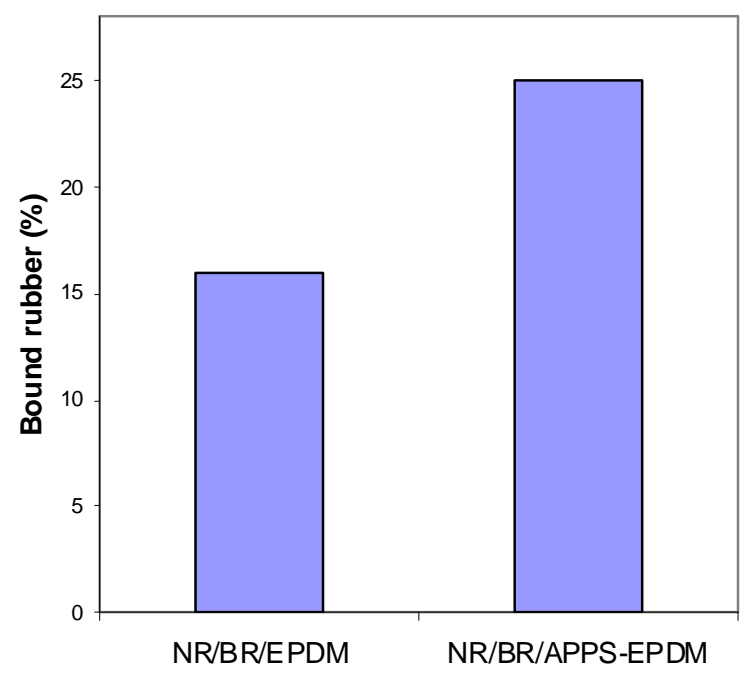

Figure 9.3 Bound rubber content of the NR/BR/EPDM blend and the NR/BR/APPS-EPDM blend (APPS5).

The slope of the stress-strain curve in a relative linear region, typically within the range of extension ratios from $100 \%$ to $300 \%$, is a good indicator for the interaction between carbon black and rubber $^{10,11}$. Therefore, a typical carbon blackrubber interaction parameter $(\sigma)$ may be calculated from following equation:

$$
\sigma=\frac{\sigma_{b}-\sigma_{a}}{\lambda_{b}-\lambda_{a}}
$$

Where $\sigma_{b}$ and $\sigma_{a}$ are the stresses at corresponding strains $\lambda_{b}: 300 \%$ and $\lambda_{a}$ : $100 \%$. The calculated $\sigma$-values for corresponding NR/BR/EPDM and NR/BR/APPSEPDM blends are shown in Table 9.4. The use of APPS-grafted EPDM shows higher $\sigma$ value compared to the virgin EPDM, and the values increase with higher APPS amounts. This result is consistent with the bound rubber measurement, confirming an enhanced carbon black-rubber interaction after APPS-grafting onto EPDM. 
Table 9.4 Carbon black-rubber interaction parameters $(\sigma)$ for NR/BR/EPDM and NR/BR/APPS-EPDM blends.

\begin{tabular}{c|ccccc}
\hline Compounds & Ref-1 & APPS-3 & APPS-5 & APPS-7 & APPS-10 \\
\hline$\sigma$ & 4.5 & 4.8 & 5.3 & 5.5 & 5.7 \\
\hline
\end{tabular}

\subsubsection{Carbon black adsorption measurements}

The improved carbon black-rubber interaction after APPS-grafting onto EPDM suggests a good affinity between carbon black and APPS. In order to further substantiate this, carbon black adsorption measurements were done for APPS and two other chemicals with similar structure, as shown in Table 9.5. Compared to APPS, phenyl disulphide and phenol disulphide are chemical compounds, without and with hydroxyl groups, respectively.

Table 9.5 Chemical structure of APPS, phenyl disulphide and phenol disulphide.

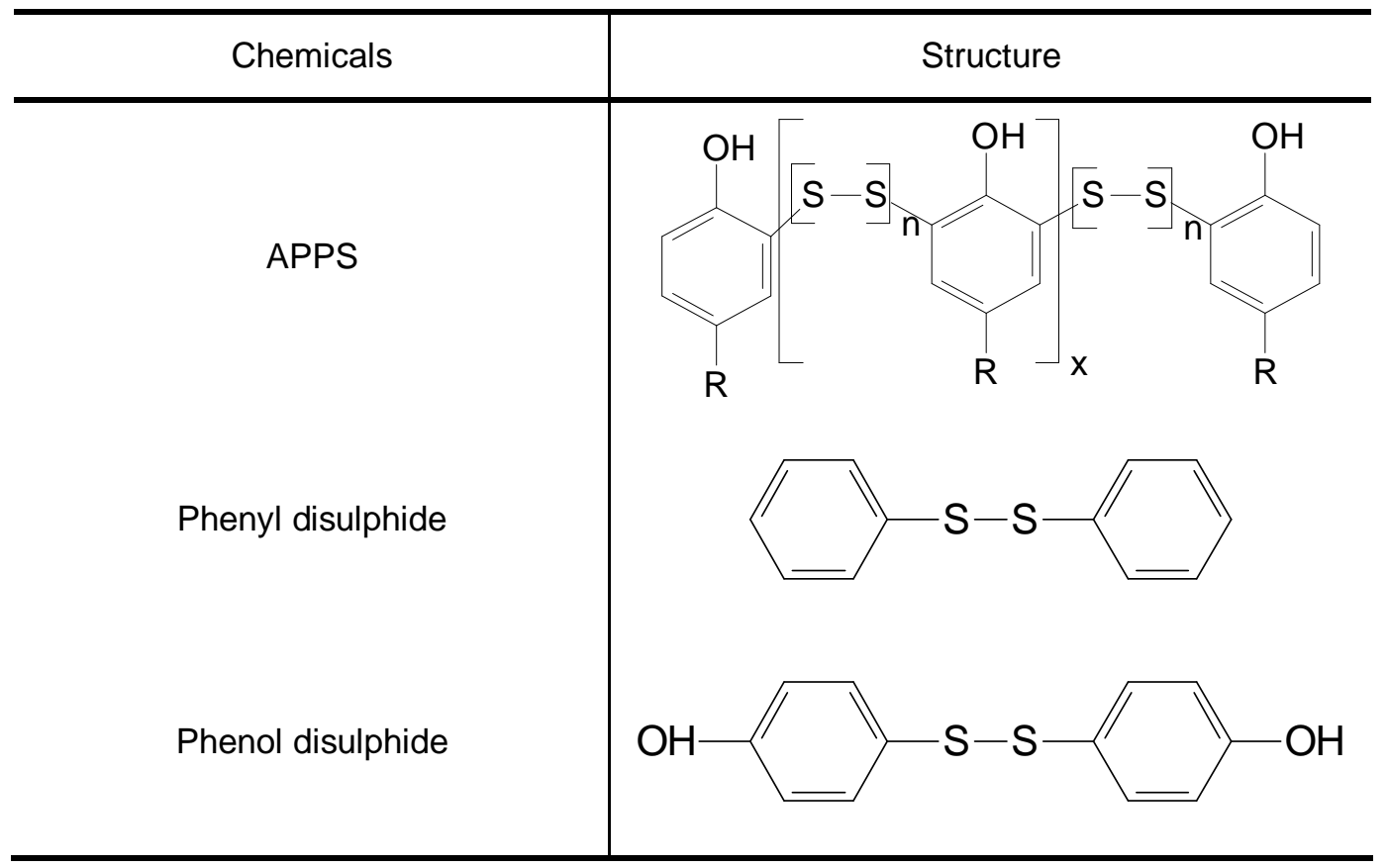

Figure 9.4 shows the weight increase of carbon black after its adsorption of phenyl disulphide, phenol disulphide and APPS. The weight increase of carbon black after adsorption of phenyl disulphide is very limited and does not increase continuously with increasing concentration. For phenol disulphide, the adsorption value increases continuously but does not reach the saturated status yet when the phenyl disulphide-concentration has been increased to $1 \mathrm{~g} / 10 \mathrm{ml}$. The adsorption value of APPS on carbon black also increases with the amount, but it reaches a plateau when the concentration of the APPS solution is $0.4 \mathrm{~g} / 10 \mathrm{ml}$. In the range of an APPS concentration of $1 \mathrm{~g} / 10 \mathrm{ml}$, the carbon black-adsorption value of phenol disulphide and APPS are similar and much higher than that of phenyl disulphide. 
The adsorption value is an indicator for the affinity between the chemicals and the carbon black. Therefore, the affinity between phenyl disulphide and carbon black is very weak. While for APPS and phenol disulphide the affinity is much stronger. Carbon black has some functional groups like hydroxyl, carboxyl, ketones, and aldehydes on the surface, even though the quantity is very small ${ }^{12,13}$, which may enhance its affinity with chemicals having hydroxyl groups. The strong affinity between APPS and carbon black is therefore consistent with the better carbon blackrubber interaction observed after the grafting of APPS onto EPDM.

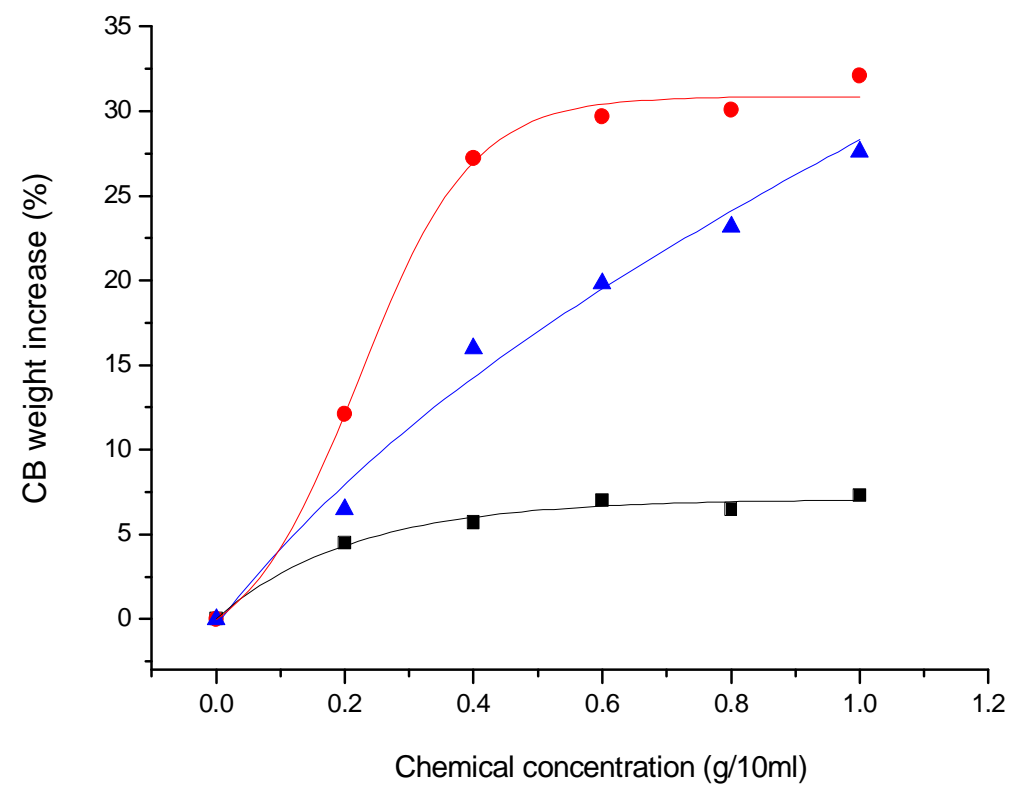

Figure 9.4 Carbon black adsorption with APPS: (I ), phenyl disulphide:(n) and phenol disulphide: $(\mathbf{\Lambda})$.

\subsubsection{Morphological characterization with TEM and STEM}

TEM was first applied to characterise the phase morphology of the NR/BR/EPDM blend: Ref-1, and the NR/BR/APPS-EPDM blend: APPS-5. The TEMimages of the gum blends without carbon black are shown in figure 9.5. In the TEM experiments, the polydiene phases NR and BR, which are more stained by osmium tetroxide than EPDM, show as dense areas with higher atomic number and appear dark in the images ${ }^{14}$. The EPDM phase contains a very small amount of double bonds in the molecule, and is by far less stained by osmium tetroxide and therefore appears light in the TEM images. It can be observed that the EPDM is dispersed in the NR/BR matrix as particles with different sizes. Comparing the images of Ref-1 and APPS-5, the differences are small. Feret's diameter, which is defined as the longest distance between any two points along the selection boundary, can be calculated according to the images as an indicator of the particle size. It was found that the average Feret's diameter for the EPDM particles of APPS-5 is $0.51 \mu \mathrm{m}$ which 
is slightly smaller than that of Ref- 1 , which is $0.62 \mu \mathrm{m}$. The particle size distributions of these two blends are shown in figure 9.6. Most EPDM particles are smaller than $0.6 \mu \mathrm{m}$ for both Ref- 1 and APPS- 5 cases. Compared to the Ref- 1 blend, the APPS- 5 blend has some larger amount of smaller particles: $<0.4 \mu \mathrm{m}$, indicating a slightly better EPDM particle distribution.

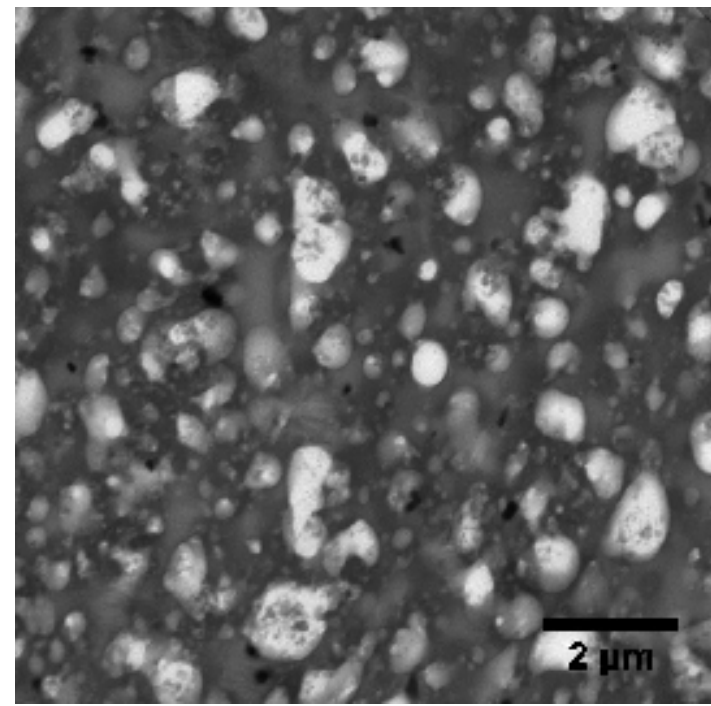

(a)

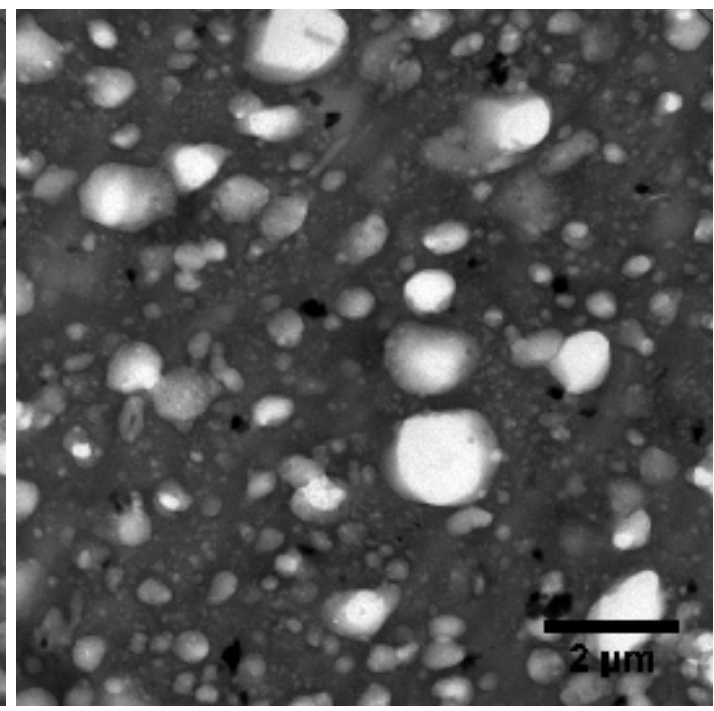

(b)

Figure 9.5 TEM images for: (a) gum Ref-1; (b) gum APPS-5;

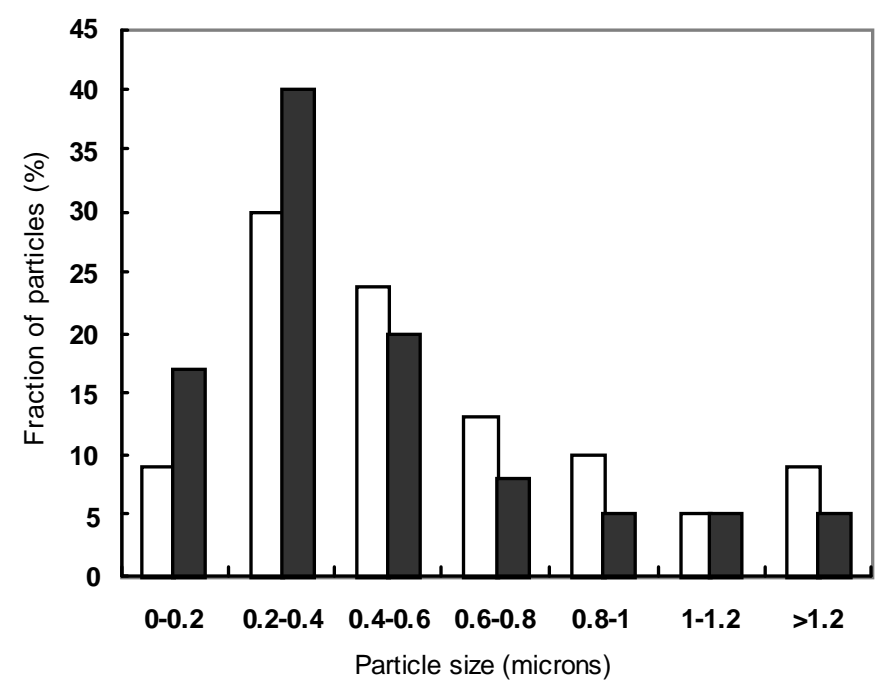

Figure 9.6 Particle size distributions for gum Ref-1: (๑), and gum APPS-5: (n).

Figure 9.7 shows the TEM-images of the HAF carbon black-filled blends of Ref-1 and APPS-5. Carbon black is heavily stained by osmium tetroxide. Therefore, the carbon black-filled area appears dark in the image. The light areas are the EPDM particles again which are not filled with carbon black. The image of APPS-5 clearly shows a more homogeneous morphology and less white areas compared to Ref-1. However, in the TEM images the carbon black phase cannot clearly be distinguished. 
Staining of the samples also has some influence on the final image or even brings about some artificial results.

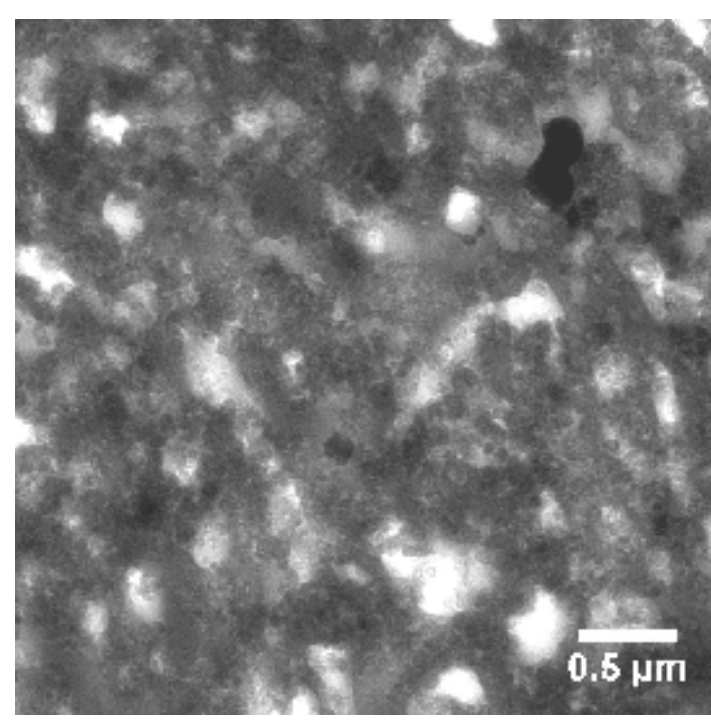

(a)

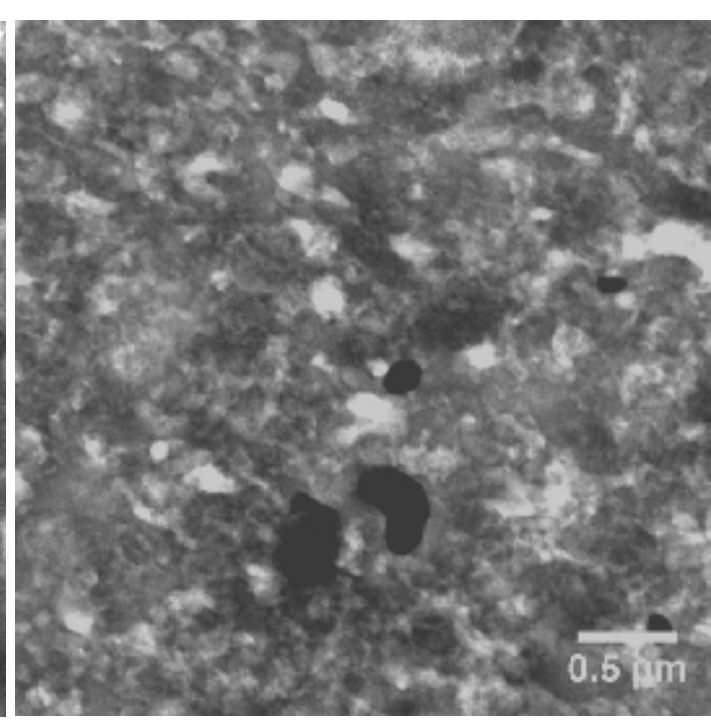

(b)

Figure 9.7TEM images for: (a) HAF-filled Ref-1; (b) HAF-filled APPS-5.

The Scanning Transmission Electron Microscope (STEM) is a special type of a transmission electron microscope: the electrons pass through the specimen, but as in scanning electron microscopy, the electron optics focus the beam onto a narrow spot which is scanned over the sample in a raster. By using STEM, especially with a highangle annular dark field (HAADF) detector, it is possible to form images where the contrast is directly related to the atomic number variations of the elements in the sample. For polymer materials, the contrast of different phases is dependent on the density of the materials ${ }^{15,16}$. The density of the major components in the blends under investigation follows such an order: carbon black $\left(1.8 \mathrm{~g} / \mathrm{cm}^{3}\right)>\mathrm{NR}\left(0.92 \mathrm{~g} / \mathrm{cm}^{3}\right)>\mathrm{BR}$ $\left(0.91 \mathrm{~g} / \mathrm{cm}^{3}\right)>\operatorname{EPDM}\left(0.86 \mathrm{~g} / \mathrm{cm}^{3}\right)$. There are several advantages with using STEM compared to TEM: it creates images with less artifacts; it is easier to be interpreted and no staining is needed ${ }^{17}$.

Figure 9.8 (a) shows the STEM image for the gum NR/BR/EPDM blend: Ref- 1 . EPDM now appears to be the dark phase and disperses as particles with clear boundaries in the image. NR appears to be the light phase. If observing the image more carefully, a gray phase can also be found with blurry boundaries, which most likely can be attributed to BR due to its slightly lower density than NR. The morphology of the gum blends observed by the STEM test is basically the same as the results from the TEM measurements: figure 9.5(a). The STEM image for carbon black in the blend is shown in figure 9.8(b). Carbon black appears the lightest phase in the blends, as its density is much higher than that of the rubbers. In this image, 
carbon black primary particles and aggregates can be clearly distinguished. The typical size of the primary carbon black particles is about $20-30 \mathrm{~nm}$.

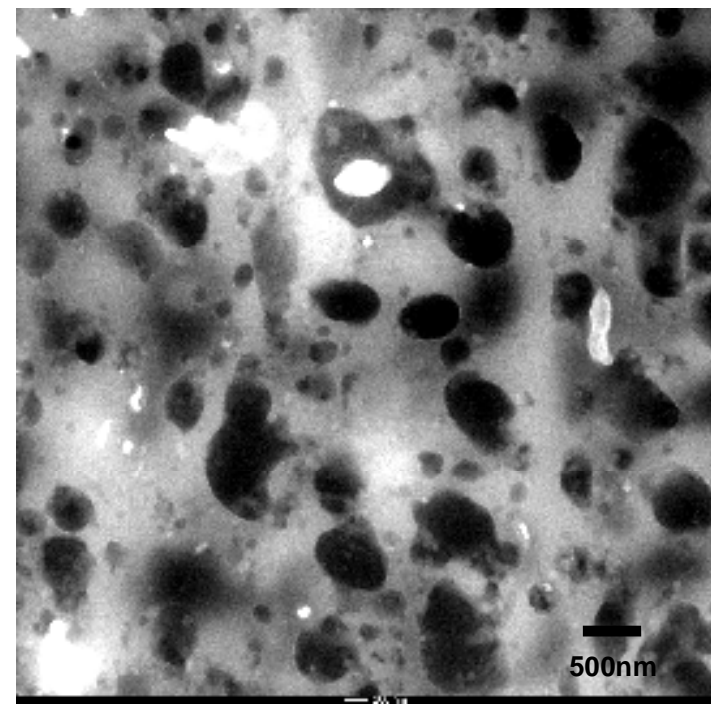

(a)

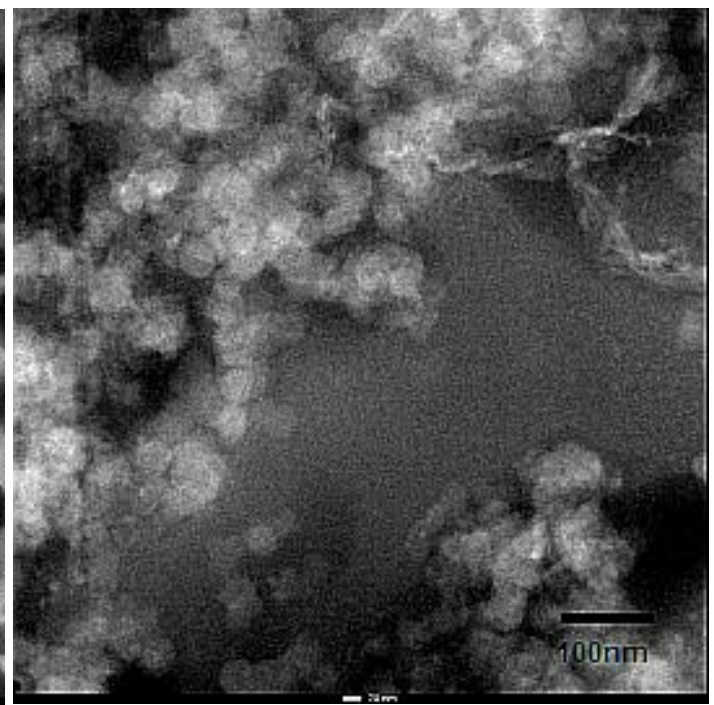

(b)

Figure 9.8 The dark field STEM images for: (a) the gum NR/BR/EPDM blend; (b) carbon black in the blend.

Figure 9.9 shows the STEM images for HAF carbon black-filled Ref-1 and APPS-5 blends, respectively. Carbon black appears as white spots in the morphology. For the image of the black-filled Ref-1 blend, there are some black areas without white spots, which can be attributed to unfilled-EPDM. While in the filled-APPS- 5 blend, the black areas are much smaller and carbon black distributes almost everywhere in the blend. Because the amount of carbon black is relatively high, and the contrast between the different rubber phases is diffused by the much larger density of carbon black relative to the rubbers, it is difficult to quantify the exact carbon black distribution in the different rubber phases.

From the STEM image of the HAF-filled-APPS-5 blend with larger magnification, as shown in figure 9.10 , it can clearly be seen that there are a lot of carbon black particles dispersed in the dark EPDM phase. It confirms that after EPDM modification with APPS, more carbon black becomes distributed into the EPDM phase, and therefore, a more homogeneous morphology is obtained as compared to the blend with unmodified EPDM. 


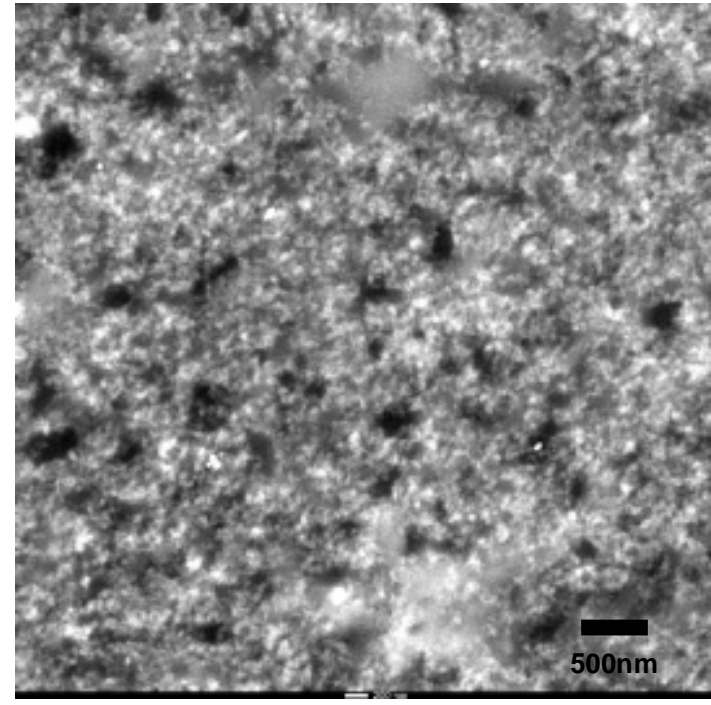

(a)

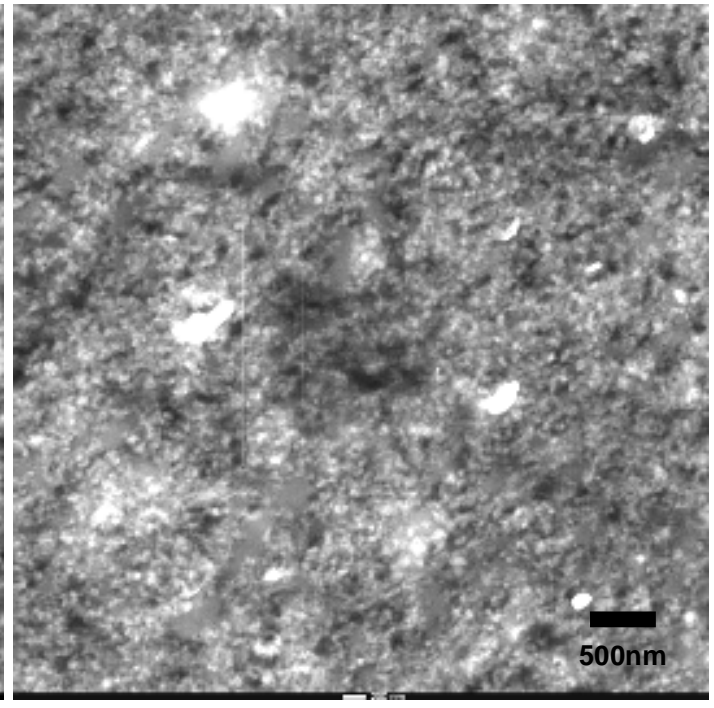

(b)

Figure 9.9 The dark field STEM images for: (a) HAF-filled Ref-1; (b) HAF-filled APPS-5.

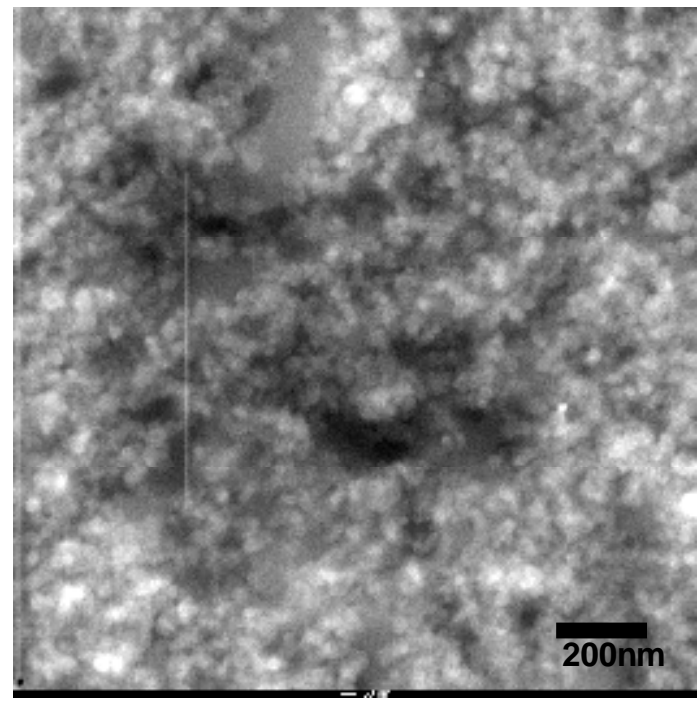

Figure 9.10 The dark field STEM image for the HAF-filled APPS-5 with larger magnification.

\subsection{Conclusions}

As demonstrated in Chapter 8, the use of APPS-grafted EPDM within the NR/BR compound for tire sidewalls, provides significant improvement of the mechanical properties. Possible mechanisms involved in this improvement were investigated. ATR/IR spectra show that modification of EPDM with APPS takes place. The modification obtains saturation for APPS amounts higher than 5wt\%. Bound rubber tests confirm a better rubber-carbon black affinity by using APPSEPDM in the blend and the better affinity is proved to be due to the hydroxyl groups in the APPS chemical structure, as concluded from the carbon black adsorption measurements. TEM and STEM images of HAF-filled NR/BR/APPS-EPDM blends 
illustrate a more homogeneous rubber phases morphology in comparison with the straight NR/BR/EPDM blends. Particularly a much more homogeneous distribution of the carbon black over the different rubber phases is held responsible for the improvement in properties of these blends.

\section{REFERENCES}

1. Zapp, R. L., Rubber Chem. Technol., 46, 251 (1973).

2. Rodgers, M. B.; Solis, S. C.; Tambe, N.; Sharma, B. B., Rubber Chem. Technol., 81, 600 (2008).

3. Solis, S. C.; Rodgers, M. B.; Tambe, N.; Sharma, B. B.; Waddell, W. H. Meeting of the Rubber Division, American Chemical Society, San Antonio, Texas, October, 2005.

4. Flowers, D. D.; Fusco, J. V.; Tracey, D. S., Rubber World, 209, 32 (1994).

5. Poljansek, I.; Krajnc, M., Acta Chim. Slov., 52, 238 (2005).

6. Holopainen, T. O.; Alvila, L.; Rainio, J.; Pakkanen, T. T., J. Appl. Polym. Sci., 69, 2175 (1998).

7. Kraus, G., Advan. Polym. Sci., 8, 156 (1971).

8. Meissner, B., J. Appl. Polym. Sci., 18, 2483 (2003).

9. Bras, J. L.; Papirer, E., J. Appl. Polym. Sci., 22, 525 (2003).

10. Ayala, J. A.; Hess, W. M.; Dotson, A. O.; Joyce, G. A., Rubber Chem. Technol., 63, 747 (1990).

11. Ayala, J. A.; Hess, W. M.; Kistler, F. D.; Joyce, G. A., Rubber Chem. Technol., 64, 19 (1991).

12. Wang, M. J., Rubber Chem. Technol., 71, 520 (1998).

13. Choi, S. S., Elastomer, 36, 37 (2001).

14. Sawyer, L. C.; Grubb, D. T., in "Polymer Microscopy", Chapman and Hall, London, 1996.

15. Heidenreich, R. D., in "Fundamentals of Transmission Electron Microscopy", Wiley, New York, 1964.

16. Loos, J.; Sourty, E.; Lu, K.; With, de G.; Bavel, S. v., Macromolecules, in press.

17. Clarke, D. R., J. Mat. Sci., 5, 689 (1970). 


\section{Summary}

Ethylene-Propylene-Diene rubber (EPDM) possesses excellent ozone, heat and ageing resistance due to its highly saturated chemical structure. It is well known that incorporation of saturated EPDM rubber into high-diene rubbers, such as natural rubber (NR) and (butadiene rubber) BR, is a way to achieve non-staining ozone resistance for tyre sidewall applications instead of using conventional antiozonants, such as N-1,3-dimethylbutyl,-N'-phenyl-para-phenylenediamine (6PPD), which have staining effects and leach out via migration. For reason of the difference in molar concentrations of double bonds and the difference in polarity, blending of EPDM with high-diene rubbers generally gives the following problems: thermodynamic incompatibility of these types of rubber, cure incompatibility and heterogeneous reinforcing filler distribution in each of the rubber phases. Therefore, vulcanisates of such elastomer blends are generally poor in mechanical properties. The objective of the project in this thesis is to overcome these incompatibility and unevenreinforcement problems of the NR/BR/EPDM blends and improve the final mechanical properties. The research background and an overview of various research approaches in the field of blending dissimilar rubbers are described in Chapter 2.

Based on a patent of Goodyear, N-Chlorothio-N-Butyl-Benzenesulphonamide (CTBBS) is selected in Chapter 3 as a representative of a general class of $\mathrm{N}$ chlorothiosulphonamides to modify EPDM in order to enhance the compatibility of EPDM in e.g. NR/BR/EPDM blends as a starting point of this project. Upon the addition of CTBBS to ethylidene norbornene-(ENB)-containing EPDM, gelation occurs and therefore a low amount of grafting is achieved. With the limited grafting efficiency for ENB-EPDM, there is no significant improvement in properties when applying the grafted ENB-EPDM into NR/BR/EPDM blends. Further studies are carried out to compare the CTBBS-modification efficiencies for two other types of EPDM: hexadiene (HD)-EPDM and dicyclopentadiene-(DCPD)-containing EPDM. Among all these three types of EPDMs, HD-EPDM shows the highest effectivity 
towards CTBBS-grafting. However, this EPDM is not commercially available anymore. On the contrary, DCPD-EPDM shows the lowest reactivity so that almost no grafting could be realized.

Adding compatibilising agents to improve the affinity of EPDM towards polydiene rubber is another approach to solve the incompatibility problems. Maleicanhydride grafted EPM (MAH-EPM) is used as compatibilising agent for the NR/BR/EPDM blends and the mechanical properties are described in Chapter 4. Straight EPM and N-phenyl-p-phenylenediamine modified MAH-EPM (NPD-EPM) are also studied for comparison. Neither straight EPM nor NPD-EPM enhances the mechanical properties of such blends. However, the mechanical properties are clearly improved by the addition of 5phr MAH-EPM. The tensile strength of the MAHEPM compatibilised blend is much higher than of the straight NR/BR/EPDM blend and even very close to the NR/BR tyre sidewall compound. Higher modulus, better trouser tear strength and slightly increased elongation at break are also obtained. An improvement of the fatigue-to-failure property is observed as well when the test was carried out with constant strain energy. The ozone resistance under both static and dynamic conditions of all the NR/BR/EPDM and NR/BR/EPDM/EPM blends with $30 \mathrm{phr}$ of EPDM/EPM is comparable with the NR/BR compounds containing 6PPD.

Chapter 5 discusses the mechanistic aspects contributing to those improvements. It is found that the polarity change of the EPDM-phase after the addition of the MAH-EPM is not significant enough to improve the solubility of curatives in the EPDM phase, to account for better curing of the EPDM moiety in the blend. TEM images of gum and HAF-filled NR/BR/EPDM/MAH-EPM blends illustrate a significantly more homogeneous reinforcing carbon black distribution between the various phases in comparison with the straight NR/BR/EPDM blends. Compression set and strain sweep measurements indicate the formation of additional crosslinks in the blends due to the ionic interaction of MAH-EPM with zinc oxide. Therefore, improved compatibility, better carbon black distribution and some effect of additional ionic crosslinking lead to the improvements of the mechanical properties by using MAH-EPM as compatibilising agent for the NR/BR/EPDM blend.

Based on the work described in Chapters 4 and 5, MAH-EPM is further modified with sulphur-containing chemicals, as $\mathrm{N}$-cyclohexyl-2-benzothiazolesulphenamide (CBS) and dithiodianiline (DTDA), to achieve co-cure of MAH-EPM with the other rubbers, which is absent for straight MAH-EPM. In Chapter 6, it is found that CBS is not grafted onto MAH-EPM as one complete molecule during the modification reaction of CBS with MAH-EPM. The benzothiazole group in CBS, which would help with the co-cure of MAH-EPM with the other rubbers in the blends, is not grafted. Then the CBS-modified MAH-EPM cannot improve the mechanical properties, as seen in the earlier studies on NPD-EPM in Chapter 4 . In the reaction of DTDA- 
modification onto MAH-EPM, modification and crosslinking take place at a same time. With 1.0eq DTDA, relatively less crosslinking and more simple modification is achieved compared to $0.5 \mathrm{eq}$. The addition of 1.0eq DTDA-modified MAH-EPM to the $\mathrm{NR} / \mathrm{BR} / \mathrm{EPDM}$ blend does not give a valuable improvement of the tensile properties as compared to unmodified MAH-EPM.

Three modification agents: CBS, 2,2'-dithiobis(pyridine-N-oxide) (PNO) and caprolactam disulfide (CLD) are summarised in Chapter 7. The reactions of these modification agents with EPDM are described, respectively. It is found that grafting of CBS onto EPDM takes place and that a mercaptobenzothiazol-rubber intermediate is generated when EPDM is heated in the presence of CBS, sulphur and $\mathrm{ZnO}$, according to a characterisation with ATR/IR. The compound is partly insoluble in a common solvent after reaction, indicating some unwanted crosslinking during the modification. For the grafting of PNO and CLD onto EPDM, different compositions, reaction temperatures and reaction time are tried. However, both chemical species are very sensitive to become crosslinked during the reaction. No evidence from ATR/IR and NMR shows that PNO and CLD are successfully grafted onto EPDM.

A new modification agent, alkylphenol polysulphide (APPS), is selected in Chapter 8. The modification reaction is carried out in an internal mixer at about $180^{\circ} \mathrm{C}$. APPS-modified EPDM is then blended with NR and BR. The physical properties of the blends with APPS-modified EPDM are significantly improved compared with compounds using virgin EPDM, and are equivalent or even somewhat superior to conventional NR/BR tyre sidewall compounds. The tensile strength and tear strength of the NR/BR/APPS-EPDM blends and the reference NR/BR tyre sidewall compound are more or less equivalent. The NR/BR/APPSEPDM blends show superior fatigue-to-failure properties to straight NR/BR/EPDM and the NR/BR tyre sidewall compound. Dynamic viscoelastic properties are also improved by using APPS-grafted EPDM compared to virgin EPDM, although there is still some room for improvement relative to the conventional NR/BR sidewall blend. All NR/BR/EPDM blends show better ageing properties relative to the NR/BR compound. But the use of APPS-grafted EPDM does not result in further improvement relative to straight EPDM.

In order to create a better understanding of the APPS grafting-reaction onto EPDM and the behaviour of APPS-grafted EPDM in blends with NR/BR, mechanistic studies are discussed in Chapter 9. ATR/IR spectra show that modification of EPDM with APPS takes place. The modification reaches saturation when the APPS-amount approaches $5 w t \%$. Bound rubber tests confirm a better rubber-carbon black affinity by using APPS-EPDM in the blend and the better affinity is proved to be due to the hydroxyl-group in the APPS-chemical structure, as can be derived from the results of carbon black adsorption measurements. TEM and STEM images of HAF carbon 
black-filled NR/BR/APPS-EPDM blends illustrate a significantly more homogeneous carbon black distribution in comparison with the straight NR/BR/EPDM blends.

In this research generally two approaches have been explored to solve the incompatibility and uneven-reinforcement problems of the NR/BR/EPDM blends and to improve the mechanical properties: adding a compatibilising agent and modification of the EPDM. Applying MAH-EPM as compatibilising agent leads to some improvements of the mechanical properties. However, ionic crosslinking resulting from $\mathrm{MAH}-\mathrm{EPM}$ with $\mathrm{ZnO}$, is not thermally stable, which limits the application at high temperatures. Different modification agents have been tried and it was found that APPS works by far most effectively. Significant improvements have been obtained with APPS-modified EPDM in blends with NR/BR. There is still some room for improvement, especially for the visco-elastic properties. But due to the excellent tensile, tear and fatigue properties, it can be a serious candidate for tyre sidewall applications. Considering the modification reaction of EPDM with APPS, the reaction mechanism is not clear yet and is worth to be further explored. 


\section{Samenvatting}

Door de hoge mate van verzadiging in de chemische structuur van EthyleenPropyleen-Dieen rubber (EPDM) bezit het een uitstekende bestendigheid tegen ozon , hitte en veroudering. Rubbers met een hoge graad van onverzadiging, zoals natuurlijk rubber (NR) en butadieen rubber (BR), die niet kleurvast en ozon-bestendig zijn, kunnen ozon-bestendig gemaakt worden door het inbouwen van deze EPDM rubber. Dit is interessant voor met name de zijvlakken van van autobanden. Hiermee kunnen de conventionele anti-ozonanten, zoals N-1,3-dimethylbutyl,-N'-phenyl-paraphenylenediamine (6PPD), die aanleiding geven tot verkleuring en die door migratie uitlogen, vervangen worden. Vanwege het verschil in concentratie aan dubbele bindingen (onverzadigdheid) en het verschil in polariteit tussen deze rubbersoorten, leidt het mengen van EPDM met rubbers met een hoge onverzadigdheid tot een niethomogeen mengsel. Dit effect leidt tot een niet-nomogenen verdeling van de versterkende vulstof in de twee rubberfasen en tot een verschil in vernetting van beide polymeren. Vulkanisaten van dergelijke elastomeermengsels hebben ten gevolge hiervan in het algemeen slechte mechanische eigenschappen. Het doel van het project, dat beschreven staat in dit proefschrift is om deze problemen van incompatibiliteit van de rubbers en de ongelijke verdeling van de versterkende vulstof in de verschillende rubberfasen te ondervangen en om daarmee de mechanische eigenschappen te verbeteren. In Hoofdstuk 2 wordt de achtergrond van het onderzoek beschreven en een overzicht gegeven van de verschillende methoden van aanpak van het onderzoek op het gebied van het verbeteren van de homogeniteit van mengsels door het veranderen van de polariteit van de EPDM.

Gebaseerd op een patent van Goodyear, is gekozen voor N-Chlorothio-NButyl-Benzenesulphonamide (CTBBS), als vertegenwoordiger van een algemene klasse van $\mathrm{N}$-chlorothiosulphonamides en dat als beginpunt dient om de compatibiliteit van EPDM in bv. NR/BR/EPDM-mengsels te bevorderen. Als CTBBS aan EPDM, dat ethylideennorborneen (ENB) als dieen bevat wordt toegevoegd, vindt gelering plaats en is hierdoor de graftingsgraad laag. Als gevolg van de beperkte graftingefficiëntie van ENB-EPDM, is er geen significante verbetering in 
eigenschappen te zien indien de gegrafte ENB-EPDM in NR/BR/EPDM-mengsels wordt gebruikt. Andere experimenten zijn uitgevoerd om de efficiënties van CTBBSmodificatie te vergelijken met twee andere typen EPDM: hexadieen (HD-)EPDM en dicyclopentadieen (DCPD-)EPDM. Van deze drie typen EPDM's, heeft HD-EPDM de grootste effectiviteit m.b.t. CTBBS-grafting. Deze EPDM is echter niet meer commercieel verkrijgbaar. Daarentegen vertoont DCPD-EPDM de laagste reactiviteit, zodat bijna geen grafting kan worden bereikt. Dit staat beschreven in Hoofdstuk 3.

Een andere aanpak om de incompatibiliteitsproblemen op te lossen is het toevoegen van compitibilizing agents om de affiniteit van EPDM t.o.v. polydieen rubber te verbeteren. In Hoofdstuk 4 wordt het gebruik van maleïne-anhydride gegrafte EPM (MAH-EPM) als compatibilizing agent voor de NR/BR/EPDM mengsels en de mechanische eigenschappen beschreven. Gewoon EPM en N-phenyl-pphenylenediamine-gemodificeerd MAH-EPM (NPD-EPM) zijn ook bestudeerd ter vergelijking. Noch gewoon EPM noch NPD-EPM verbetert de mechanische eigenschappen van zulke mengsels. De mechanische eigenschappen verbeteren echter duidelijk door toevoeging van 5 phr MAH-EPM. De treksterkte van het mengsel dat met MAH-EPM is gecompatibiliseerd, is veel hoger dan die van het gewone NR/BR/EPDM mengsel en ligt zelfs dicht tegen die van de NR/BRcompound die wordt gebruikt voor de zijvlakken van een band. Hogere modulus, betere trouser scheursterkte en ook een lichte toename in breuk bij rek wordt bereikt. $\mathrm{Er}$ is een verbetering van de vermoeidheidseigenschap te zien als de proef werd uitgevoerd met constante strain energie. De ozonbestendigheid van alle NR/BR/EPDM- en NR/BR/RPDM/EPM-mengsels met $30 \mathrm{phr}$ EPDM/EPM, onder zowel statische als dynamische omstandigheden is vergelijkbaar met de NR/BRcompounds die 6PPD bevatten.

In Hoofdstuk 5 worden de mechanistische aspecten besproken die tot die verbeteringen bijdragen. Het blijkt dat de polariteitsverandering van de EPDM-fase na toevoeging van MAH-EPM niet significant genoeg is om de oplosbaarheid van de curatives in de EPDM-fase te verbeteren. Dus een betere vulkanisatie van het EPDM deel in het mengsel kan hiermee niet verklaard worden. TEM foto's van gum NR/BR/EPDM/MAH-EPM-mengsel en NR/BR/EPDM/MAH-EPM-mengsels die met HAF (High Abrasion Furnace black) vulmiddel zijn versterkt, laten een duidelijk homogenere verdeling van het versterkende roet zien tussen de verschillende fasen in vergelijking met de gewone NR/BR/EPDM megsels.

Dus, verbeterde compatibiliteit, betere roet verdeling en een zeker effect van extra ionogene crosslinking leidt tot verbeteringen van de mechanische eigenschappen door MAH-EPM als compatibiliserende agens voor NR/BR/EPDM mengsel.

Gebaseerd op het werk dat beschreven is in Hoofstuk 4 en 5, is MAH-EPM verder gemodificeerd met zwavel bevattende chemicaliën zoals $\mathrm{N}$-cyclohexyl-2- 
benzothiazolesulphenamide (CBS) en dithiodianiline (DTDA) om co-vulcanisatie van MAH-EPM met de andere rubbers te bewerkstelligen. Co-vulcanisatie is afwezig voor gewone MAH-EPM.

In Hoofdstuk 6 blijkt dat CBS niet gegraft wordt op MAH-EPM als één volledige molecuul, tijdens de modificatie reactie van CBS met MAH-EPM.

De benzothiazol groep in CBS, die de co-vulcanisatie van MAH-EPM zou bevorderen, wordt niet gegraft. Daarom kan de CBS gemodificeerde MAH-EPM de mechanische eigenschappen niet verbeteren, zoals te zien is Hoofdstuk 4 bij eerdere studies van NPD-EPM.

Bij de modificatie reactie van DTDA aan MAH-EPM, vindt modificatie en crosslinking tegelijkertijd plaats.

Met 1.0 eq DTDA vindt relatief minder crosslinking plaats en meer gewone modificatie in vergelijking tot 0.5 eq.

Toevoegen van 1.0 eq DTDA gemodificeerde MAH-EPM aan NR/BR/EPDM mengsel geeft geen waardevolle verbetering van de trek eigenschappen in vergelijking tot de ongemodificeerde MAH-EPM.

In Hoofdstuk 7 worden drie modificatie agentia, CBS, 2,2'-dithiobis(pyridine-N-oxide) (PNO) and caprolactam disulfide (CLD) samengevat. De reacties van deze modificatie agentia met EPDM worden respectievelijk besproken. Er wordt gevonden dat graften van CBS op EPDM plaatsvindt en dat een mercaptobenzothiazol-rubber intermediair wordt gevormd als EPDM wordt verwarmd in aanwezigheid van CBS, zwavel en $\mathrm{ZnO}$, zoals gevonden uit karakterisatie met ATR/IR. De verbinding is na reactie gedeeltelijk oplosbaar in een gewoon oplosmiddel, wat duidt op een ongewenste vrosslinking gedurende de modificatie. Voor de grafting van PNO en CLD op EPDM. Zijn verschillende samenstellingen, reactie temperaturen en reactie tijden geprobeerd. Beide chemische stoffen zijn echter erg gevoelig voor crosslinking tijdens de reactie. Er zijn met ATR/IR en NMR geen aanwijzingen gevonden dat PNO en CLD op EPDM gegraft worden.

In Hoofdstuk 8 wordt een nieuwe modificatie agens, alkylphenol polysulfide (APPS), geïntroduceerd en besproken. De modificatie reactie wordt uitgevoerd in een interne menger bij ongeveer $180 \mathrm{C}$. Hierbij wordt APPS-gemodificeerd EPDM gemengd met NR en BR. De fysische eigenschappen van de mengsels met APPS-gemodificeerd EPDM zijn aanzienlijk verbeterd, vergeleken met de compounds waarbij virgin EPDM is gebruikt en gelijkwaardig of zelfs iets beter dan van de conventionele compounds die worden gebruikt voor NR/BR-zijvlakken van banden.

Om de APPS graft reactie op EPDM en het gedrag van APPS-gegrafte EPDM in mengsels met NR/BR beter te begrijpen, worden in Hoofdstuk 9 mechanistische studies besproken. ATR/IR spectra laten zien dat modificatie van EPDM met APPS plaatsvindt. De modificatie bereikt verzadiging (is maximaal?!) bij een APPS 
concentratie van ca. 5 gew \%. Bound rubber proeven bevestigen een beter rubberroet affiniteit als APPS-EPDM in het mengsel wordt gebruikt. Deze verbeterde affiniteit blijkt toegeschreven te kunnen worden aan de hydroxyl-groep in de APPSchemische structuur, zoals kan worden afgeleid uit de resultaten van roet adsorptie metingen. TEM en STEM beelden van HAF roet-gevulde NR/BR/APPS-EPDM mengsels laten een aanzienlijk homogenere roet verdeling zien in vergelijking met de normale NR/BR/EPDM mengsels.

In dit onderzoek zijn voornamelijk twee aanpakken onderzocht om de problemen m.b.t. de incompatibiliteit en de ongelijke versterking van de NR/BR/EPDM mengsels op te lossen en om de mecahnische eigenschappen te verbeteren door toevoeging van compatibiliserend agens en modificatie van het EPDM. Gebruik van MAH-EPM als compatibiliserende agens leidt tot enige verbetering van de mechanische eigenschappen. lonogene crosslinking als gevolg van reactie van MAH-EPM met $\mathrm{ZnO}$ echter, is niet thermisch stabiel. Hierdoor is gebruik bij hogere temperaturen beperkt. Verschillende modificatie agentia zijn geprobeerd en het blijkt dat APPS verreweg het meest effectieve is. Aanmerkelijke verbeteringen zijn bereikt met APPS-gemodificeerde EPDM in mengsels met NR/BR. Er is nog wel wat te verbeteren, in het bijzonder m.b.t. de visco-elastische eigenschappen. De excellente trek-,scheur- en vermoeidheideigenschappen maken dat het een serieuze kandidaat kan zijn voor gebruik in zijvlakken van banden. Het reactiemechanisme van de modificatie van EPDM met APPS is echter nog niet duidelijk en moet verder worden onderzocht. 


\section{Symbols and Abbreviations}

\begin{tabular}{|c|c|}
\hline 6PPD & $\mathrm{N}$-(1, 3-dimethylbutyl)-N'-phenyl-p-phenylenediamine \\
\hline APPS & Alkylphenol polysulfide \\
\hline ATR/IR & Attenuated total reflectance infrared spectroscopy \\
\hline BAPD & bis-Alkylphenoldisulphide \\
\hline BDR & Bound rubber amount \\
\hline BIMS & Brominated isobutylene-co-para-methyl styrene \\
\hline BR & Butadiene rubber \\
\hline C & Concentration of diffusing matter \\
\hline C.E.D. & Cohesive energy density \\
\hline CBS & N-cyclohexyl- 2-benzothiazolesulfenamide \\
\hline CIIR & Chlorobutyl rubber \\
\hline CLD & Caprolactam disulfide \\
\hline CR & Chloroprene rubber \\
\hline CTAB & Cetyl trimethyl ammonium bromide \\
\hline CTBBS & N-chlorothio-N-butyl-benzenesulphonamide \\
\hline $\mathrm{D}$ & Diffusion coefficient \\
\hline DBP & Dibutylphtalete \\
\hline DCPD & Dicyclopentadiene \\
\hline$\Delta \mathrm{H}_{\mathrm{m}}$ & Enthalpy of mixing of two liquids \\
\hline DIPDIS & Bis(diisopropyl) thiophosphoryl disulphide \\
\hline DTDA & Dithiodianiline \\
\hline DTDC & Dithiodicaprolactam \\
\hline DTDM & Dithiodimorpholine \\
\hline ENB & Ethylidene norbornene \\
\hline EPDM & Ethylene-propylene-diene rubber \\
\hline G" & Loss modulus \\
\hline$G^{\prime}$ & Storage modulus \\
\hline
\end{tabular}




$\begin{array}{ll}\text { HAF } & \text { High abrasion furnace black } \\ \text { HD } & \text { Hexadiene } \\ H_{V} & \text { Molar heat of vaporization } \\ \text { IIR } & \text { Butyl rubber } \\ \text { IR } & \text { Synthetic polyisoprene } \\ \text { MAH-EPM } & \text { Maleic-anhydride modified EPM } \\ \text { MBT } & \text { Mercaptobenzothiazole } \\ \text { NBR } & \text { Nitriel rubber } \\ \text { NMR } & \text { Nuclear magnetic resonance } \\ \text { NPD-EPM } & \text { N-phenyl-p-phenylenediamine modified MAH-EPM } \\ \text { NPPDA } & \text { N-phenyl-p-phenylenediamine } \\ \text { NR } & \text { Natural rubber } \\ \text { phr } & \text { Parts per hundred rubber } \\ \text { PNO } & \text { 2,2'-Dithiobis(pyridine-N-oxide) } \\ \text { PPD } & \text { Para-phenylene diamine } \\ \text { PVC } & \text { Polyvinylchloride } \\ \text { Q } & \text { Permeation coefficient } \\ \text { q } & \text { Amount of diffusing substance passing a cross section of } \\ \text { RPA } & \text { surface area A } \\ \text { S } & \text { Rubber process analyser } \\ \text { SBR } & \text { Solubility coefficient } \\ \text { STEM } & \text { Styrene-butadiene rubber } \\ \text { T } & \text { Scanning transmission electron microscopy } \\ \text { Tan } \delta & \text { Loss angle } \\ & \end{array}$




\section{Curriculum vitae}

Hongmei Zhang was born in Jiangsu Province, China on January $28^{\text {th }}, 1981$. In 2002, she obtained her BE degree in Applied Chemistry at Shanghai Jiao Tong University. In the same year, she started her master's studies at the same university. One and a half years later, she was recommended to attend a cooperation programme between Shanghai Jiao Tong University and University of Twente. She came to the Netherlands and joined the Rubber Technology Group (RBT) in January 2004 as a master student. She obtained her MSc degree from University of Twente in December 2004, with her master thesis entitled "Investigation on the effects of 1,2-polybutadiene as a co-agent in dynamically vulcanised thermoplastic elastomers." In May 2005 she started her Ph.D work in the same group on the project: "Investigation on blending, reinforcement and curing in NR/BR/EPDM rubber compounds for the tyre sidewall application". The research was funded by Dutch Polymer Institue (DPI). The results of this research are described in this thesis. 


\section{Acknowledgements}

Time is flying! When I look back to these years in the Netherlands, it passed by much faster than I could have imagined. But I believe this period must be one of the most memorable periods in my life. Before completing this dissertation, I owe profound debt of gratitude to many people who have greatly contributed to or have helped with the development of this research in their special ways.

First of all, I am especially grateful to my promoter Prof. J.W.M. Noordermeer for giving me the opportunity to carry out a master project, and later on to become a Ph.D. student in your research group. Thank you very much for your enlightening guidance and endless encouragement during all the time of the research of the project and the writing of this thesis, without which, this work could not have been accomplished. Actually you have helped me not only to develop the research skills but also to improve my writing, presentation and other personal skills, which must be a great treasure for my future.

Secondary, I would express my deepest regards to my assistant promoter, Dr. R.N. Datta. You are such a nice supervisor and good friend to me. I will never forget all the invaluable advices, constant encouragement and warm care you gave me during the last four years. Full of sorrow that you are not possible to be there at my promotion anymore, but I believe you will see it in the heaven. You will always be in my heart!

I would like to thank the other members of my promotion committee for making the effort to read this thesis and provide me with valuable comments.

I wish to acknowledge the Dutch Polymer Institute (DPI) for the financial support of this research project. I am also grateful to all my DPI committee members: Dr. J.E. Stamhuis (DPI), Dr. M. van Dui (DSM), Dr, P. Meessen (DSM), Dr. M. Alvarez Grima (DSM), Mr. S. Barbera (SKF). Thank you all for your nice discussions in these committee meetings and thank you for your kind cooperation.

It is my great pleasure to thank ERT B.V. for helping with the fatigue to failure and the ozone resistance tests. I wish to thank Mr. Konijnendijk (Arkema) for providing the Vultac samples for my research. I would also like to acknowledge the cooperation of Dr. 
Kangbo Lv (TU/e), Joachim Loos (TU/e) and Mark Smithers for the help regarding TEM and STEM measurements.

Special thanks to Auke for your valuable advices and a lot of help in the chemistry related issues. Thank you for helping me with correcting the Samenvatting part. Thank you also for giving me a lot of support after Rabin's passing.

Kannika and Jacob: thank you very much for being my paranimffs. Thank you for your warm friendship and kind cooperation. Dear Jacob, special thanks to you for making the translation of the summary into Dutch. I will remember your "fatherly advices".

Dear Rui, it is my luck to have you as one of my best friends from the very beginning moment when we arrive in the Netherlands. Thanks for your supporting and sharing all along. We will be good friends forever.

I want to thank all the other RBT/ETE colleagues for your help and friendship: Wilma, Yvoone, Gerda, Aleida, Hillie, Kinsuk, Pratip, Vipin, Francesca, Montse, Marek, Wilco, Kuno, Tony, Anoma, Mukund, Agata, Satoshi, Sreekumar, Morteza, Siti. I also appreciate all the nice time we spent together beside working, like golf, bowling, karting, farmer games and of course our group trip to Poland.

I also extend my gratitude to all members from PBM and MTP for help.

My Chinese friends: Rui Guo, Yan Song, Jing Song, Wei Zhou, Wei Zhao, Lanti Yang, Hao Gu, Yujie Ma, Qi Chen, Rong Jin, Chao Lin, Zhipeng Hu, Hui Lv, Yang Zhang, Wei Bai, Chunlin Song, Tian Gang, Xin Wan, thank you all for your friendship and a lot of help. I will remember all the wonderful time we spent together: chatting, having nice dinners, poker games, KaraOK, travelling... Thank you for making my life in Enschede more colourful.

Finally, I would like to take this opportunity to express my deepest thanks to my parents for their unconditional support and love. I owe my loving thanks to my husband Feng. We had to stay apart for five years due to my research abroad. It is not easy for both of us, but we managed. Dear Feng, without your constant understanding and support, it would be impossible for me to finish this work. 